\title{
Tractable Stochastic Analysis in High Dimensions via Robust Optimization
}

\author{
by \\ Chaithanya Bandi \\ B.Tech, Indian Institute of Technology, Chennai (2008) \\ Submitted to the Sloan School of Management \\ in partial fulfillment of the requirements for the degree of \\ Doctor of Philosophy in Operations Research \\ at the \\ MASSACHUSETTS INSTITUTE OF TECHNOLOGY \\ June 2013 \\ ARCHIVES

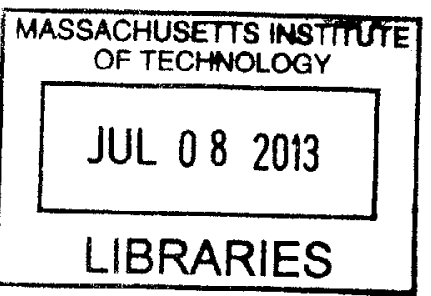 \\ (c) Massachusetts Institute of Technology 2013. All rights reserved.
}

Author

Sloan School of Management

May 17, 2013

Certified by

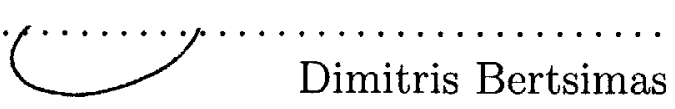

Boeing Leaders for Global Operations Professor

Co-director, Operations Research Center

Thesis Supervisor

1

Accepted by...$\ldots \ldots \ldots \ldots \ldots$

v Patrick Jaillet

Co-director, Operations Research Center 


\title{
Tractable Stochastic Analysis in High Dimensions via Robust Optimization
}

\author{
by \\ Chaithanya Bandi

\begin{abstract}
Submitted to the Sloan School of Management on May 17, 2013, in partial fulfillment of the requirements for the degree of Doctor of Philosophy in Operations Research
\end{abstract}

\begin{abstract}
Modern probability theory, whose foundation is based on the axioms set forth by Kolmogorov, is currently the major tool for performance analysis in stochastic systems. While it offers insights in understanding such systems, probability theory, in contrast to optimization, has not been developed with computational tractability as an objective when the dimension increases. Correspondingly, some of its major areas of application remain unsolved when the underlying systems become multidimensional: Queueing networks, auction design in multi-item, multi-bidder auctions, network information theory, pricing multi-dimensional financial contracts, among others. We propose a new approach to analyze stochastic systems based on robust optimization. The key idea is to replace the Kolmogorov axioms and the concept of random variables as primitives of probability theory, with uncertainty sets that are derived from some of the asymptotic implications of probability theory like the central limit theorem. In addition, we observe that several desired system properties such as incentive compatibility and individual rationality in auction design and correct decoding in information theory are naturally expressed in the language of robust optimization. In this way, the performance analysis questions become highly structured optimization problems (linear, semidefinite, mixed integer) for which there exist efficient, practical algorithms that are capable of solving problems in high dimensions. We demonstrate that the proposed approach achieves computationally tractable methods for (a) analyzing queueing networks (Chapter 2) (b) designing multi-item, multi-bidder auctions with budget constraints, (Chapter 3) (c) characterizing the capacity region and designing optimal coding and decoding methods in multi-sender, multi-receiver communication channels (Chapter 4).
\end{abstract}

Thesis Supervisor: Dimitris Bertsimas

Title: Boeing Leaders for Global Operations Professor

Co-director, Operations Research Center 


\section{Acknowledgments}

First and foremost, I would like to thank my academic advisor, Dimitris Bertsimas for their phenomenal support and guidance over the course of my doctoral work at MIT. Apart from academic research, Dimitris played a remarkable role in my personal development throughout my stay at MIT, and I would be ever grateful to him for that. It has been an absolute privilege interacting with him, both in his office as well as outside.

I would like to thank the other two members of my thesis committee, Georgia Perakis and Vivek Farias, who helped me greatly in improving this work. Their constant encouragement was crucial for me to carry on. I would also like to thank every ORC faculty member as its their involvement that makes the ORC such a unique environment and I consider myself extremely fortunate for being given an opportunity to be a part of it. I would also like to thank Laura and Andrew for their constant support during my entire $\mathrm{PhD}$.

Apart from academics, at ORC I have met some of the nicest people and in the process have made life lasting friends. I would like to thank Shubham Gupta, Michael Frankovich, Nikos Trichakis, Yuan Zhong, Nataly Youssef, Yehua Wei, Joline, Shashi Mittal whom I have worked with both academically as well to have fun. I would also like to thank the every other ORC student who make ORC such a friendly place to be at. Outside of ORC, I would like to thank Sujith, Vinay, Raj, Samuel, George and many others who provided the non-work circle of friends.

Finally, I would like to thank my parents and aunt without whom I would not exist. And I dedicate my thesis to their support throughout. 


\section{Contents}

1 Introduction $\quad \mathbf{1 5}$

1.1 A Critical View of the History of Probability Theory . . . . . . . 15

1.2 The Building Blocks of our Approach . . . . . . . . . . . . . . . . 19

1.2.1 The Connection with Optimization .......... 20

1.2 .2 Constructing Uncertainty Sets . . . . . . . . . . . 21

1.3 Overview of the Thesis . . . . . . . . . . . . 25

2 Robust Queueing Theory 29

2.1 The New Primitives . . . . . . . . . . . . . . . . . . 34

2.1.1 The Robust Queue Model . . . . . . . . . . . . . . . . 34

2.1 .2 Construction of Uncertainty Sets . . . . . . . . . 35

2.2 The Multi-Server Robust Queue . . . . . . . . . . . . . . . 37

2.2.1 Waiting Time in a Single-Server Robust Queue . . . . . 38

2.2.2 Waiting Time in a Multi-Server Robust Queue . . . . . . . . 42

2.2 .3 Implications and Insights . . . . . . . . . . . 46

2.3 Departure Process of Robust Queues . . . . . . . . . . . . . 48

2.3.1 Departure Process in a Single-Sever Robust Queue . . . . . 48

2.3.2 Departure Process in a Multi-Sever Robust Queue . . . . . . . 49

2.3 .3 Implications and Insights . . . . . . . . . . . . . 51

2.4 The Robust Queueing Network Analyzer . . . . . . . . . . . . . 52

2.4 .1 The Superposition Process . . . . . . . . . . . . . . 53

2.4 .2 The Thinning Process ............... 56

2.4.3 The Overall Network Characterization . . . . . . . . . . 57 
2.5 Queues with Asymmetric Heavy-tailed Arrival and Service Processes . 60

2.6 Computational Results . . . . . . . . . . . . . . . 63

2.6.1 Derived Variability Parameters ... . . . . . . . . . 64

2.6 .2 The RQNA Algorithm . . . . . . . . . . . . . . . 66

2.6.3 Performance of RQNA in Comparison to QNA and Simulation 68

2.6.4 Performance of RQNA as a Function of Network Parameters . 68

2.7 Concluding Remarks $\ldots \ldots \ldots$. . . . . . . . . . . . . .

$\begin{array}{lll}3 & \text { Robust Optimal Auctions } & \mathbf{7 3}\end{array}$

3.1 Auction Theory . . . . . . . . . . . . . . . . . 73

3.1.1 Relevant Literature . . . . . . . . . . . . . . . 74

3.1 .2 Motivation and Contributions ........... 77

3.1.3 Structure of the Chapter and Notation . . . . . . . . . . 79

3.2 Model and Problem Formulation . . . . . . . . . . . . . . . . . 79

3.2.1 The Robust Optimization Approach . . . . . . . . . . . . 80

$3.2 .2 \quad$ A Robust Optimal Mechanism . . . . . . . . . . . . 82

$3.2 .3 \quad$ Solving $R O M \ldots \ldots \ldots \ldots \ldots$

3.2 .4 The Case of Indivisible Items $\ldots \ldots \ldots$

3.3 Single Item Auctions . . . . . . . . . . . . . . . . . 100

3.3.1 The Robust Optimal Mechanism for Single Item Auctions without Budget Constraints . . . . . . . . . . . . . . . . 101

3.3.2 Closed Form Solutions . . . . . . . . . . . . . . . . 107

3.3.3 Comparison with the Myerson Auction . . . . . . . . . . . 109

3.4 Multi-Item Auctions with Private Budgets . . . . . . . . . . . 113

3.4.1 The Robust Optimal Mechanism for Auctions with Budget Uncertainty . . . . . . . . . . . . . . 115

3.5 Computational Results . . . . . . . . . . . . . . . . . 115

3.5.1 Computational Tractability . . . . . . . . . . . 118

3.5.2 Effect of Correlations on the Revenue . . . . . . . . . . . . . 119

3.6 Summary and Conclusions . . . . . . . . . . . . . . . . . . . . 119 
4 Network Information Theory $\quad 123$

4.1 Introduction . . . . . . . . . . . . . . . . 123

4.1.1 Problem Definition and Notation ... . . . . . . 125

4.1 .2 Relevant Literature . . . . . . . . . . . . . . . 126

4.1.3 Structure of the chapter . . . . . . . . . . . . . 128

4.2 Maximum Likelihood Decoding and Typical Sets . . . . . . . . . . . . 129

4.2.1 Optimality of Maximum Likelihood Decoder . . . . . . . . . 129

4.3 The Single User Gaussian Channel . . . . . . . . . . . . . . 130

4.3.1 An Optimization Formulation of the Coding Problem . . . . . 131

4.3.2 Correctness of Algorithm 1 . . . . . . . . . . . . 139

4.4 Capacity Region of a Gaussian Interference Channel . . . . . . . . . 152

4.4.1 Capacity Characterization and Optimal Coding . . . . . . . 154

4.4.2 Correctness of Algorithm 4 . . . . . . . . . . . . . 162

4.4.3 Remarks on the Optimality of the Decoder (4.48) . . . . . 175

4.5 Channels with Exponential Noise . . . . . . . . . . . . . 176

4.5.1 Single User Exponential Channel - Capacity Characterization and Optimal Coding . . . . . . . . . . . . 176

4.5.2 Two-User Exponential Interference Channel - Capacity Characterization and Optimal Coding . . . . . . . . 179

4.6 The Multi-access Channel and the Multi-cast Channel . . . . . . . . . 180

4.6.1 Capacity Region of a Gaussian Multi-access channel (MAC) . 180

4.6 .2 Multi-cast Channel . . . . . . . . . . . . . 186

4.7 Implementation and Computational Results . . . . . . . . . . . 193

4.7.1 Computational Experiments : Gaussian Interference Channel . 193

4.7.2 Computational Experiments : Exponential Channel . . . . . . 197

4.8 Conclusion . . . . . . . . . . . . . . . . . . . . . . . . . 198

$\begin{array}{llr}5 & \text { Conclusion } & 199\end{array}$ 


\section{List of Figures}

2-1 Dynamics of a Two-Sever Queue. . . . . . . . . . . . . . . . . . 43

3-1 Calculation of the reservation prices and the nominal allocations. . . 84

3-2 Calculation of adapted allocations and payments. . . . . . . . 85

3-3 Generalized Benders Decomposition Algorithm for Problem (3.8). . . 99

3-4 Mechanism for Indivisible Items. . . . . . . . . . . . . . . . 100

3-5 Calculation of the reservation price. . . . . . . . . . . . . . . . 102

3-6 Calculation of allocations and payments. . . . . . . . . 103

3-7 The Second Price Auction with a reservation price. . . . . . . . . . 104

3-8 Robustness of ROM-Si. . . . . . . . . . . . . . . . . . . . . . . . . . . 112

3-9 Calculation of the reservation prices and the nominal allocations. . . 116

3-10 Calculation of adapted allocations and payments. . . . . . . . . 117

3-11 Effect of Correlations on the Revenue. . . . . . . . . . . . . . . 120

4-1 Comparison with Carleial's Rate Region . . . . . . . . . . . . 196

4-2 Comparison with Han-Kobayashi Rate Region . . . . . . . . . . . . . 196

4-3 Two-user Gaussian interference channel $-P_{1}=10, P_{2}=20, h_{12}=$

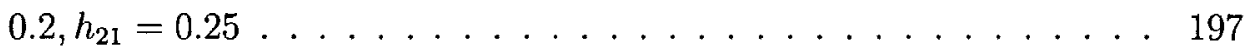




\section{List of Tables}

2.1 Adapted $\theta$ values. . . . . . . . . . . . . . . . . . 65

2.2 Percentage errors for single server queues using RQNA. (* Service Independent ${ }^{* *}$ Service Dependent $\ldots \ldots 66$

2.3 Percentage errors for multi-server queues using RQNA. . . . . . . . . 66

2.4 Percentage errors on Kuehn's network using RQNA. (* Service Independent $\quad{ }^{* *}$ Service Dependent $\ldots \ldots \ldots$. . . . . . . . 69

2.5 Sojourn time percentage errors of RQNA relative to simulation as a function of the size of the network and the degree of feedback for queues with single server queueing nodes. . . . . . . . . . . . . . . 69

2.6 Sojourn time percentage errors of RQNA relative to simulation as a function of the size of the network and the degree of feedback for queues with multi-server queueing nodes. . . . . . . . . . . . . 70

2.7 Sojourn time percentage errors for RQNA relative to simulation as a function of the maximum traffic intensity and the number of distinct distributions for the external arrival processes for single server queueing networks. . . . . . . . . . . . . . . .

2.8 Sojourn time percentage errors for RQNA relative to simulation as a function of the maximum traffic intensity and the number of distinct distributions for the external arrival processes for multi-server queueing

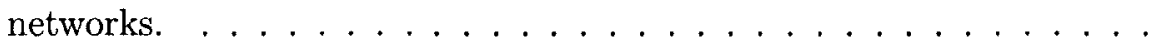

3.1 Myerson vs ROM-Si : The Relative Revenue, defined in (3.67) for different distributions with the same mean and standard deviation. . 
3.2 Myerson vs ROM-Si: The Relative Revenue under the same mean but different standard deviation. . . . . . . . . . . . . . . 111

3.3 Myerson vs ROM-Si : The Relative Revenue under the same standard deviation but different mean . . . . . . . . . . . . . . . 111

3.4 Comparison of reservation prices when valuations obey $N(\mu, \sigma)$. . 113

3.5 Computational times (in minutes) of ROM-Si for uncorrelated valuations. 118

3.6 Computational times (in minutes) of ROM-Si for correlated valuations with correlation coefficient equal to $0.2 \ldots \ldots \ldots 118$

3.7 Computational times (in minutes) of ROM-Si for correlated valuations with coefficient equal to $0.4 \ldots \ldots \ldots \ldots$. . . . . . . . . . . . .

4.1 Dependence of the nature of the optimization problem with noise distribution. ........................ 176 


\section{Chapter 1}

\section{Introduction}

In this chapter we provide an overview of our non-probabilistic approach to model uncertainty in a system. We start by presenting a brief history of Probability Theory with an emphasis on the difficulties associated with performance analysis of systems modeled using Probability Theory. The discussion of these difficulties allows us to motivate our approach in the context of modeling uncertainty in complex systems. We then describe the main machinery of our approach with appropriate examples.

\subsection{A Critical View of the History of Probability Theory}

Probability theory has a long and distinguished history that dates back to the beginning of the 17th century. Games involving randomness led to an exchange of letters between Pascal and Fermat in which the fundamental principles of probability theory were formulated for the first time. The Dutch scientist Huygens, learned of this correspondence and in 1657 published the first book on probability entitled $D e$ Ratiociniis in Ludo Aleae. In 1812 Laplace introduced a host of new ideas and mathematical techniques in his book Theorie Analytique des Probabilities. Laplace applied probabilistic ideas to many scientific and practical problems. The theory of errors, actuarial mathematics, and statistical mechanics are examples of some of the important 
applications of probability theory developed in the $19^{\text {th }}$ century. Many researchers have contributed to the theory since Laplace's time; among the most important are Chebyshev, Markov, von Mises, and Kolmogorov.

One of the difficulties in developing a mathematical theory of probability has been to arrive at a definition of probability that is precise enough for use in mathematics, yet comprehensive enough to be applicable to a wide range of phenomena. The search for a widely acceptable definition took nearly three centuries. The matter was finally resolved in the 1933 monograph of Kolmogorov who outlined an axiomatic approach that forms the basis for the modern theory. With the publication in 1933 of Kolmogorov's book Grundbegriffe der Wahrscheinlichkeitsrechnung, Kolmogorov laid the foundations of an abstract theory, designed to be used as a mathematical model for certain classes of observable events. The fundamental concept of the theory is the concept of a probability space $(\Omega, A, P)$, where $\Omega$ is a space of points $\omega$ which are denoted as elementary events, while $A$ is a $\sigma$-algebra of sets in $\Omega$, and $\mathrm{P}$ is a probability measure defined for all $A$-measurable events, i.e., for all sets $S$ belonging to $A$. Kolmogorov's three axioms form the basis of this theory

1. $P(S) \geq 0, \forall S \in A$.

2. $P(\Omega)=1$.

3. If $S_{i} \in A, i \geq 1$, are pairwise disjoint, then $P\left(\cup_{i=1}^{\infty} S_{i}\right)=\sum_{i=1}^{\infty} P\left(S_{i}\right)$.

Another important primitive of probability theory is the notion of a random variable as a quantity that takes values with certain probabilities. A key objective of probability theory is to estimate the probability distributions of a random variable $Y$, which is a function of $n$ primitive random variables $X_{1}, \ldots, X_{n}$, that is $Y=f\left(X_{1}, \ldots, X_{n}\right)$, given information on the joint probability distribution of the primitive random variables $X_{1}, \ldots, X_{n}$. For example, suppose that we are given $n$ independent, random variables $X_{i}$ uniformly distributed in $[0,1]$, and we are interested in evaluating the distribution the random variable $Y=\sum_{i=1}^{n} X_{i}$. Specifically, we are interested in the quantity $P(Y \leq t), 0 \leq t \leq n$. Even for a modest value of $n=10$, this is a 
complex calculation involving convolutions. Perhaps the simplest way to calculate the Laplace transform of $Y$, which is the product (because of independence) of the Laplace transforms of the $X_{i}$ 's and then numerically invert the transform. Note that in order to estimate a probability of a relative simple event, we need to invoke rather heavy machinery from complex analysis and inverse of transforms.

The situation we described is not an isolated instance. Consider a single class queueing network (see Chapter 2), that has been used in the latter part of the $20^{\text {th }}$ century to model computer and communication networks. Suppose we are interested in the expected value of the number of jobs waiting in one of the queues in the network. If the distribution of interarrival and service times is not exponential, we do not know how to calculate this expectation exactly, and two avenues available to make progress are simulation and approximation. Simulation can take a considerable amount of time in order for the results to be statistically significant, and in addition, if the simulation model is complex as it is often the case, then it is difficult to isolate and understand the key insights in the model. On the other hand, approximation methods can potentially lead to results that are not very close to the true answers. Given these considerations, it is fair to say that after more than 50 years of research we really do not have a satisfactory answer to the problem of performance analysis of queueing networks. Kingman [2009], one of the pioneers of queueing theory in the $20^{\text {th }}$ century in his opening lecture at the conference entitled " 100 Years of QueueingThe Erlang Centennial", writes, "If a queue has an arrival process which cannot be well modeled by a Poisson process or one of its near relatives, it is likely to be difficult to fit any simple model, still less to analyze it effectively. So why do we insist on regarding the arrival times as random variables, quantities about which we can make sensible probabilistic statements? Would it not be better to accept that the arrivals form an irregular sequence, and carry out our calculations without positing a joint probability distribution over which that sequence can be averaged?' .

The situation in queueing networks we discussed above is present in other examples. Shannon [1948b] characterized the capacity region and designed optimal coding and decoding methods in single-sender, single-receiver channels, but the extension to 
multi-sender, multi receiver channels with interference is by and large open. Myerson [1981], in his nobel prize winning work, solved the problem of optimal market design in single item auctions, but the extension to multi-item case with bidders that have budget constraints has remained open. Black and Scholes [1973], in their nobel prize winning work, solved the problem of pricing an option in an underlying security, but the extension to multiple securities with market frictions has not been resolved. In all of these and other problems, we see that we can solve the underlying problem in low dimensional problems, but we have been unable to solve the underlying problems when the dimension increases.

In our opinion, the reason for this is related to the history of probability theory as a scientific field. The multi-century effort that led to the the development of modern probability theory aimed to lay the conceptual and foundational basis of the field. The primitives of probability theory, the Kolmogorov axioms and the notion of a random variable, while powerful for modeling purposes, have not been developed with computational tractability as an objective when the dimension increases. In contrast, consider the development of optimization as a scientific field in the second part of the $20^{\text {th }}$ century. Modern linear optimization originated by Dantzig [1949] had from the beginning of the field as an objective to solve multi-dimensional problems computationally. Today, we can solve truly large scale structured optimization problems (linear, conic, mixed integer).

Given the success of optimization to solve multi-dimensional problems, it is natural, in our opinion, to formulate probability problems as optimization problems. For this purpose, we utilize robust optimization, arguably one of the fastest growing areas of optimization in the last decade, to accomplish this. In this effort, we are guided by the words of Dantzig [1963], who in the opening sentence of his book Linear Programming and Extensions writes "The final test of any theory is its capacity to solve the problems which originated it." 


\subsection{The Building Blocks of our Approach}

One of the major successes of probability theory is the development of limit laws. As an illustration consider the central limit theorem that asserts that if $X_{i}, i=1, \ldots, n$ are independent, identically distributed random variables with mean $\mu$ and standard deviation $\sigma$, then as $n \rightarrow \infty$, the random variable $S_{n}=\sum_{i=1}^{n} X_{i}$ is asymptotically distributed as a standard normal, that is

$$
\lim _{n \rightarrow \infty} P\left(\frac{S_{n}-n \mu}{\sigma \sqrt{n}} \leq t\right)=P(Z \leq t)
$$

where $Z$ is a random variable that obeys the normal distribution with mean 0 and standard deviation of 1 , denoted as $Z \sim N(0,1)$. The importance of the limit laws in the theory of probability can be emphasized by quoting Kolmogorov (Gnedenko and Kolmogorov [1968]): "All epistemological value of the theory of probability is based on this: that large scale random phenomena in their collective action create strict, non random regularity."

The key building block in our approach is that rather than assuming as primitives the axioms of probability theory (Kolmogorov axioms and the notion of a random variable), we assume as primitives the conclusions of probability theory, namely its limit laws. Let us give a motivating example. From the central limit theorem

$$
\frac{S_{n}-n \mu}{\sigma \sqrt{n}}
$$

is asymptotically standard normal. We know that a standard normal $Z$ satisfies

$$
P(|Z| \leq 2) \approx 0.95, P(|Z| \leq 3) \approx 0.99
$$

We therefore assume that the quantities $X_{i}$ take values such that

$$
\left|\sum_{i=1}^{n} X_{i}-n \mu\right| \leq \Gamma \sigma \sqrt{n}
$$


where $\Gamma$ is a small numerical constant 2 or 3 that is selected adaptively to make good fit empirically. In other words, we do not describe the uncertain quantities $X_{i}$ as random variables, rather they take values in an uncertainty set

$$
\mathcal{U}=\left\{\left(x_{1}, \ldots, x_{n}\right)|| \sum_{i=1}^{n} x_{i}-n \mu \mid \leq \Gamma \sigma \sqrt{n}\right\}
$$

In specific situations we can augment the uncertainty set $U$ by using additional asymptotic laws as we illustrate in Section 1.2.2.

\subsubsection{The Connection with Optimization}

Suppose we are interested in estimating $E\left[f\left(X_{1}, \ldots, X_{n}\right)\right]$, where $\left(X_{1}, \ldots, X_{n}\right)$ are random variables. Using asymptotic laws of probability, we construct an uncertainty set $\mathcal{U}$. We have already seen an example in Eq. (1.1). Then, we estimate $E\left[f\left(X_{1}, \ldots, X_{n}\right)\right]$ by solving the constrained optimization problems

$$
\begin{aligned}
& \max f\left(x_{1}, x_{2}, \ldots, x_{n}\right) \\
& \text { s.t. }\left(x_{1}, x_{2}, \ldots, x_{n}\right) \in \mathcal{U},
\end{aligned}
$$

and

$$
\begin{aligned}
& \min f\left(x_{1}, x_{2}, \ldots, x_{n}\right) \\
& \text { s.t. }\left(x_{1}, x_{2}, \ldots, x_{n}\right) \in \mathcal{U} .
\end{aligned}
$$

In other words, we transform the performance analysis question to a constrained optimization problem, arguably a problem we can solve efficiently in high dimensions, and we use the asymptotic laws of probability theory, arguably the most insightful aspect of probability theory, to construct the constrained set in the optimization problem.

Suppose that we are interested in a design problem involving design parameters $x=\left(x_{1}, \ldots, x_{n}\right) \in X$ and uncertain parameters $\theta=\left(\theta_{1}, \ldots, \theta_{n}\right)$ and we are interested 
in solving the problem

$$
\max _{x \in X} E[f(x, \theta)]
$$

We model the uncertainty of the parameters $\theta$ by the uncertainty set $\mathcal{U}$ where the parameters $\theta$ take values and solve the robust optimization problem

$$
\max _{x \in X} \min _{\theta \in \mathcal{U}} f(x, \theta)
$$

Robust Optimization ( $\mathrm{RO}$ ) is one of the fastest growing areas of optimization in the last decade. It addresses the problem of optimization under uncertainty, in which the uncertainty model is not stochastic, but rather deterministic and set-based. RO models are typically tractable computationally, but may lead to solutions that are too conservative. To alleviate conservatism, Ben-Tal and Nemirovski [2000, 1998, 1999], El-Ghaoui and Lebret [1997], and El-Ghaoui et al. [1998], proposed linear optimization models with ellipsoidal uncertainty sets, whose robust counterparts correspond to quadratic optimization problems. Bertsimas and Sim $[2003,2004]$ proposed RO models with polyhedral uncertainty sets that can model linear/integer variables, and whose robust counterparts correspond to linear/integer optimization models. For a more thorough review we refer the reader to Ben-Tal et al. [2009], and Bertsimas et al. [2011].

\subsubsection{Constructing Uncertainty Sets}

In this section, we outline the principles for constructing uncertainty sets we use in this paper.

\section{Using Historical Data and the Central Limit Theorem}

Suppose that we have estimated the mean $\mu$ and the standard deviation $\sigma$ of i.i.d. random variables $\left(x_{1}, \ldots, x_{n}\right)$. We expect that the central limit theorem holds, and we model uncertainty by the uncertainty set given in Eq. (1.1). 


\section{Modeling Correlation and ARCH models}

Consider the random variables $\mathbf{x}=\left(x_{1}, \ldots, x_{n}\right)$ which are correlated. Specifically, suppose that there are $m<n$ i.i.d. random variables $\mathbf{y}=\left(y_{1}, \ldots, y_{m}\right)$ with mean $\mu_{y}$ and standard deviation $\sigma_{y}$ such that $\mathbf{x}=\mathbf{A y}+\boldsymbol{\epsilon}$, where $\mathbf{A}$ is an $n \times m$ matrix and $\epsilon=\left(\epsilon_{1}, \ldots, \epsilon_{n}\right)$ is a vector of i.i.d. random variables that have mean zero and standard deviation $\sigma_{\epsilon}$. Then, we construct the uncertainty set given by

$$
\mathcal{U}^{\text {Corr }}=\left\{\mathbf{x}|\mathbf{x}=\mathbf{A y}+\boldsymbol{\epsilon},| \sum_{i=1}^{m} y_{i}-m \mu_{y}\left|\leq \Gamma \sigma_{y} \sqrt{m},\right| \sum_{i=1}^{n} \epsilon_{i} \mid \leq \Gamma \sigma_{\epsilon} \sqrt{n}\right\} .
$$

Using the same approach, we also model autocorrelated returns. For instance, consider an $\operatorname{AR}(q)$ model given by

$$
y_{t}=a_{0}+a_{1} \cdot y_{t-1}+\ldots+a_{q} \cdot y_{t-q}+\epsilon_{t}
$$

where the return at time $t$ depends on the returns of the previous $q$ periods and $\epsilon_{t}$ 's are i.i.d noise. To model this, we construct the uncertainty set given by

$$
\mathcal{U}^{\mathrm{AR}(q)}=\left\{\mathrm{y}\left|y_{t}=a_{0}+a_{1} \cdot y_{t-1}+\ldots+a_{q} \cdot y_{t-q}+\epsilon_{t}, \forall t,\right| \sum_{t=1}^{T} \epsilon_{t} \mid \leq \Gamma \sigma_{\epsilon} \sqrt{T}\right\} .
$$

\section{Stable Laws}

The central limit theorem belongs to a broad class of weak convergence theorems. These theorems express the fact that a sum of many independent random variables tend to be distributed according to one of a small set of stable distributions. When the variance of the variables is finite, the stable distribution is the normal distribution. In particular, these stable laws allow us to construct uncertainty sets for heavy-tailed distributions.

Theorem 1. (Nolan [1997]) Let $Y_{1}, Y_{2}, \ldots$ be a sequence of i.i.d. random variables, with mean $\mu$ and undefined variance. If $Y_{i} \sim Y$, where $Y$ is a stable distribution with 
parameter $\alpha \in(0,2)$ then

$$
\frac{\sum_{i=1}^{n} Y_{i}-n \mu}{n^{1 / \alpha}} \sim Y .
$$

Motivated by this result, one can construct an uncertainty set $\mathcal{U}^{\mathrm{HT}}$ representing the random variables $\left\{Y_{i}\right\}$ as follows

$$
\mathcal{U}^{\mathrm{HT}}=\left\{\left(z_{1}, z_{2}, \ldots, z_{n}\right) \mid-\Gamma \leq \frac{\sum_{i=1}^{n} z_{i}-n \mu}{n^{1 / \alpha}} \leq \Gamma\right\}
$$

where $\Gamma$ can be chosen based on the distributional properties of the random variable $Y$. Note that $\mathcal{U}^{\mathrm{HT}}$ is again a polyhedron.

\section{Incorporating Distributional Information}

In this section, we illustrate how to construct uncertainty sets that utilize knowledge of the specific probability distribution. We use the idea of a typical set $\mathcal{U}^{\text {Typical }}$, introduced by Shannon [1948b] in the context of his seminal work in information theory. Specifically, a typical set has two properties:

(a) $\mathbb{P}\left[\tilde{\mathbf{z}} \in \mathcal{U}^{\text {Typical }}\right] \rightarrow 1$, as $n \rightarrow \infty$.

(b) The conditional pdf $h(\tilde{\mathbf{z}})=f\left(\tilde{\mathbf{z}} \mid \tilde{\mathbf{z}} \in \mathcal{U}^{\text {Typical }}\right)$ satisfies:

$$
\left|\frac{1}{n} \log h(\tilde{\mathbf{z}})+H_{f}\right| \leq \epsilon_{n}
$$

for some $H_{f}$ (the entropy of the distribution) and $\epsilon_{n} \rightarrow 0$, as $n \rightarrow \infty$.

Property (a) means that the typical set has probability nearly 1, while Property (b) means that all elements of the typical set are nearly equiprobable, see Cover and Thomas [2006]. In order to illustrate this idea with a concrete example, consider a normal distribution $N(\mu, \sigma)$ distribution with mean $\mu$ and standard deviation $\sigma$. In 
this case, the typical set is given by

$$
\mathcal{U}^{\text {Normal }}=\left\{\left(z_{1}, \ldots, z_{n}\right) \mid n \sigma^{2}-\Gamma \leq \sum_{j=1}^{n}\left(z_{j}-\mu\right)^{2} \leq n \sigma^{2}+\Gamma\right\}
$$

More generally, for a probability density $f(\cdot)$, the typical set is given by

$$
\mathcal{U}^{\mathrm{f} \text {-Typical }}=\left\{\tilde{\mathbf{z}} \mid-\Gamma \leq \frac{\sum_{j=1}^{n} \log f\left(z_{j}\right)+n \cdot H_{f}}{\sigma_{f} \cdot \sqrt{n}} \leq \Gamma .\right\}
$$

where

$$
\begin{aligned}
H_{f} & =-\int_{-\infty}^{\infty} f(x) \log f(x) \mathrm{d} x \\
\sigma_{f} & =\int_{-\infty}^{\infty} f(x)\left(\log f(x)+H_{f}\right)^{2} \mathrm{~d} x
\end{aligned}
$$

Note that $H_{f}$ is the entropy of the random variable with pdf $f(\cdot)$. We next show that $\mathcal{U}^{\mathrm{f} \text {-Typical }}$ indeed satisfies the properties (a) and (b) of a typical set above, when $\Gamma$ is appropriately chosen.

Proposition 2. $\mathcal{U}^{\text {f-Typical }}$ satisfies

(a) $\mathbb{P}\left[\tilde{\mathbf{z}} \in \mathcal{U}^{\text {f-Typical }}\right] \rightarrow g(\Gamma)=2 \Phi(\Gamma)-1$, as $n \rightarrow \infty$.

(b) The conditional pdf $h(\tilde{\mathbf{z}})=f\left(\tilde{\mathbf{z}} \mid \tilde{\mathbf{z}} \in \mathcal{U}^{\text {f-Typical }}\right)$ satisfies:

$$
\left|\frac{1}{n} \log h(\tilde{\mathbf{z}})+H_{f}\right| \leq \epsilon_{n},
$$

with $\epsilon_{n} \rightarrow 0$, as $n \rightarrow \infty$.

Proof. (a) Since the random variables

$$
\tilde{u}_{j}=\log f\left(\tilde{z}_{j}\right)
$$


are independent and identically distributed, we apply the central limit theorem to obtain that as $n \rightarrow \infty$,

$$
\frac{\sum_{j=1}^{n} \tilde{u}_{j}+n H_{f}}{\sigma_{f} \cdot \sqrt{n}} \sim N(0,1)
$$

from where part (a) follows.

(b) Let $\tilde{\mathbf{z}} \in \mathcal{U}^{\text {f-Typical }}$. Then,

$$
h(\tilde{\mathbf{z}})=f\left(z_{1}\right) f\left(z_{2}\right) \ldots f\left(z_{n}\right) .
$$

Therefore, since $\tilde{\mathbf{z}} \in \mathcal{U}^{\text {f-Typical }}$, we have

$$
\left|\frac{1}{n} \log h(\tilde{\mathbf{z}})+H_{f}\right|=\left|\frac{1}{n} \sum_{j=1}^{n} \log f\left(z_{j}\right)+H_{f}\right| \leq \frac{\Gamma \cdot \sigma_{f}}{\sqrt{n}} \rightarrow 0,
$$

as $n \rightarrow \infty$.

\subsection{Overview of the Thesis}

In this thesis, we use the proposed approach in the following three areas:

\section{Queueing Theory.}

In Chapter 2, we use our approach for studying queueing systems (see Bandi et al. [2011]). Instead of modeling arrivals and services as renewal processes, we model the queueing systems primitives using uncertainty sets. In this framework, we obtain closed form expressions for the steady-state waiting times in multi-server queues with heavy-tailed arrival and service processes. These expressions are not available under traditional stochastic queueing theory for heavy-tailed processes, while they lead to the same qualitative insights for independent and identically distributed arrival and service times. We also develop an exact calculus for analyzing a network of queues with multiple servers based on the following key principle: a) the departure from a queue, $b$ ) the superposition, and $c$ ) the thinning of arrival processes have the same 
uncertainty set representation as the original arrival processes. We show that our approach, which we call the Robust Queueing Network Analyzer (RQNA) a) yields results with error percentages in single digits (for all experiments we performed) relative to simulation, $b$ ) performs significantly better than the Queueing Network Analyzer (QNA) proposed in Whitt [1983], and $c$ ) is to a large extent insensitive to the number of servers per queue, the network size, degree of feedback, traffic intensity, and somewhat sensitive to the degree of diversity of external arrival distributions in the network.

\section{Mechanism Design.}

In Chapter 3, we revisit the auction design problem for multi-item auctions with budget constrained buyers using our approach (see Bandi and Bertsimas [2011]). In this context, the auctioneer's beliefs on the buyers' valuations of the items are modeled by uncertainty sets. Moreover, we also leverage the fact that many core concepts such as incentive compatibility and individual rationality are naturally expressed in the language of robust optimization. In this setting, we formulate the auction design problem as a robust optimization problem and provide a characterization of the optimal solution as an auction with reservation prices, thus extending the work of Myerson [1981] from single item without budget constraints, to multiple items with budgets, potentially correlated valuations and uncertain budgets. Unlike the Myerson auction where the reservation prices do not depend on the item, the reservation prices in our approach are a function of both the bidder and the item. We propose an algorithm for calculating the reservation prices by solving a bilinear optimization problem which, although theoretically difficult in general, is numerically tractable. Moreover, this bilinear optimization problem reduces to a linear optimization problem for auctions without budget constraints and the auction becomes the classical second price auction. We report computational evidence that suggests the proposed approach (a) is numerically tractable for large scale auction design problems, (b) leads to improved revenue compared to the classical probabilistic approach when the true distributions are different from the assumed ones, and (c) leads to higher revenue when correlations in the buyers' valuations are explicitly modeled. 


\section{Information Theory.}

In Chapter 4, we consider the central problem of network information theory of characterizing the capacity region and constructing matching optimal codes for multi-user channels with interference (see Bandi and Bertsimas [2012b]). We formulate this problem as a robust optimization problem by combining the ideas of a typical sets and maximum likelihood decoding. For a single user Gaussian channel, we recover the known capacity region and at the same time construct the matching optimal code. The underlying optimization problem becomes a semidefinite optimization problem with rank one constraints. For a multi-user channel with interference, we characterize the capacity region and construct the matching optimal code for the Gaussian interference, the multi-cast and the multi-access channels by solving a semidefinite optimization problem with rank one constraints. We further examine how the probability description of noise affects the nature of the corresponding optimization problem, and show that for channels with exponentially distributed noise, the optimization problem becomes a binary, mixed linear optimization problem. While the underlying optimization problems are NP-hard, we report numerical results that show that the approach based on semidefinite optimization is computationally tractable for message book sizes of up to 100,000 .

In all these applications, we have implemented the proposed approach and have included in the thesis tables and figures with computational evidence in concrete examples in order to show that the approach of stochastic analysis based on optimization is capable of solving problems numerically in ways that, in our opinion, go beyond the current state of the art of stochastic analysis (see Bandi and Bertsimas [2012a]). The types of optimization problems that were required to be solved ranged from linear and semidefinite to discrete, bilinear and rank optimization problems. We anticipate that this research program, in addition to advancing stochastic analysis, will also advance optimization as it will reveal new optimization problems that need to be addressed. 


\section{Chapter 2}

\section{Robust Queueing Theory}

The origin of queueing theory dates back to the beginning of the $20^{\text {th }}$ century, when Erlang [1909] published his fundamental paper on congestion in telephone traffic. In addition to formulating and solving several practical problems arising in telephony, Erlang laid the foundations for queueing theory in terms of the nature of assumptions and techniques of analysis that are being used to this day. In the second part of the $20^{\text {th }}$ century, a very substantial literature of queueing theory was developed modeling queueing primitives as renewal processes.

From the time of Erlang, the Poisson process has played a very significant role in modeling the arrival process of a queue. When combined with exponentially distributed service times, the resulting $M / M / m$ queue with $m$ servers is tractable to analyze in steady-sate. While exponentiality leads to a tractable theory, assuming general distributions, on the other hand, yields considerable difficulty with respect to performing a near-exact analysis of the system. The $G I / G I / m$ queue with independent and generally distributed arrivals and services is, by and large, intractable. Currently, there does not exist a method that is capable of producing accurate numerical answers, let alone closed form expressions, for arbitrary distributions. The most general method, due to Pollaczek [1957], analyzes the performance of the $G I / G I / m$ queue by formulating a multi-dimensional problem in the complex plane. Gall [1998] portrays the exceptional difficulty of explicitly characterizing the equations for the $G I / G I / m$ queue given that their "partial solution can only be derived after long and 
complex calculations involving multiple contour integrals in a multi-dimensional complex plane". When arrival and service distributions have rational Laplace transforms of order $p$ (for example Coxian distributions with $p$ phases), the $G I / G I / m$ problem becomes intractable for higher order $p$ values. Bertsimas [1990] reports numerical results for queues with up to 100 servers and $p=2$ by finding all $h=\left(\begin{array}{c}m+p-1 \\ m\end{array}\right)$ complex roots to distinct polynomial equations and solving a linear system of dimension $h$. The system's dimension, however, increases to 4.5 million when $p=5$, hence illustrating the complexity of the problem under these assumptions.

The situation becomes even more challenging if one considers analyzing the performance of queueing networks. A key result that allows generalizations to networks of queues is Burke's theorem (Burke [1956]) which states that the departure process from an $M / M / m$ queue is Poisson. This property allows one to analyze queueing networks and leads to product form solutions as in Jackson [1957]. However, when the queueing system is not $M / M / m$, the departure process is no longer a renewal process, i.e., the interdeparture times are dependent. With the departure process lacking the renewal property, the state-of-the-art theory provides no means to determine performance measures exactly, even for a simple network with queues in tandem. The two avemues in such cases are simulation and approximation. Simulation can take a considerable amount of time in order for the results to be statistically significant. In addition, simulation models are often complex, which makes it difficult to isolate and understand key qualitative insights. On the other hand, approximation methods can potentially lead to results that are not very close to the true answers.

Given these challenges, it is fair to say that the key problem of performance analysis of queueing networks has remained open under the probabilistic framework. Motivated by this, we propose to use the uncertainty set based framework to model queueing systems based on optimization theory. Our robust optimization approach to queueing theory bears philosophical similarity with the deterministic network calculus approach which was pioneered by Cruz [1991a,b] (see also Gallager and Parekh [1994], El-Taha and Stidham [1999], C.S.Chang [2001], Boudec and Thiran [2001]). Both methods (a) take a non-probabilistic approach by placing deterministic con- 
straints on the traffic flow and (b) derive bounds on key queueing performance measures via a worst-case paradigm. There has also been a significant literature on what is called stochastic network calculus. See Jiang and Liu [2008], Jiang [2012], Ciucu et al. [2005], Burchard et al. [2011] for an overview. We note, however, that the primitives of stochastic network calculus are in fact probabilistic, so the similarity, even at the philosophical level, is significantly smaller. To a lesser degree, there is also philosophical similarity (in that it is a deterministic and worst-case approach) with adversarial queueing theory (Borodin et al. [2001], Gamarnik [2003, 2000], Goel [1999]) which was developed for stability analysis in multi-class queueing networks. In contrast, our aspiration in this work is to develop a theory of performance analysis, and thus there is no overlap between adversarial and robust queueing theory beyond the philosophical level. Beyond their deterministic and worst-case paradigms, significant differences can be noted when comparing our framework to the network calculus approach.

(a) Different Underlying Assumptions: While both methods postulate deterministic constraints over the arrival process, the assumptions are different in nature. The deterministic network calculus bounds the number of external arrivals $n_{t}$ up to time $t$ by $n_{t} \leq \lambda \cdot t+B$, where $\lambda$ denotes the traffic rate and $B$ is a constant accounting for burstiness. In contrast, our assumption on the arrival process yields different bounds on the number of arrivals $n_{t}$. In fact, denoting the arrival time of the $n_{t}^{\text {th }}$ job by $t$, i.e., $\sum_{i=1}^{n_{t}} T_{i}=t$, and applying Assumption 1(a) with tail coefficient $\alpha_{a}=2$, we obtain $n_{t}-\sqrt{n_{t}} \lambda \Gamma_{a} \leq \lambda t \leq n_{t}+\sqrt{n_{t}} \lambda \Gamma_{a}$, where $\Gamma_{a}$ represents the effect of variability. Writing $\delta^{2}=n_{t}$ yields $\delta^{2}-\lambda \Gamma_{a} \delta \leq$ $\lambda t \leq \delta^{2}+\lambda \Gamma_{a} \delta$. This implies that $\delta \geq\left(-\lambda \Gamma_{a}+\sqrt{\lambda^{2} \Gamma_{a}^{2}+4 \lambda t}\right) / 2$, leading to $n_{t} \geq \lambda t-t^{\frac{1}{2}} \lambda^{\frac{3}{2}} \Gamma_{a}$. Similarly, we obtain $n_{t} \leq \lambda t+t^{\frac{1}{2}} \lambda^{\frac{3}{2}} \Gamma_{a}$, which results in the following bounds on the number of arrivals by time $t$

$$
\left|n_{t}-\lambda \cdot t\right| \leq \Gamma_{a} \lambda^{3 / 2} t^{1 / 2}
$$

Note that the way we handle variability is different from the deterministic net- 
work calculus, and is motivated and indeed consistent with the limit laws of probability (see subsection 2.1.1).

(b) Tighter Bounds for single server queues: It is widely believed that the network calculus approach can provide overly conservative bounds for singleserver queues. In the words of Ciucu and Hohlfeld [2010] "The deterministic network calculus can lead to conservative bounds because many of the statistical properties of the arrivals are not accounted for," and for the stochastic network calculus "(only) in $\mathrm{M} / \mathrm{M} / 1$ and $\mathrm{M} / \mathrm{D} / 1$ queuing scenarios where exact results are available, the stochastic network calculus bounds are reasonably accurate," (see also Ciucu [2007]). Our approach, however, provides a bound on the waiting time for single-server queues that is qualitatively similar to its probabilistic counterpart (see subsection 2.2.1). Our computations further show that, by constraining nature via bounding the variability allowed in our uncertainty sets, we obtain results within often $2-3 \%$, and at most $8 \%$ in stochastic queueing networks (see Section 7).

(c) Generalizability: Our approach generalizes the analysis to more complex queueing systems such as multi-server queues (see subsection 2.2.2) and queueing networks with feedback (see subsection 2.4). However, "for $G I / G I / m,(m>1)$, stochastic network calculus based analysis remains plain blank" and "feedback analysis is perhaps the most critical open challenge for stochastic network calculus", as remarked by Jiang [2012]. Furthermore, while the stochastic network calculus has recently addressed heavy tails in a single-server setting (see Burchard et al. [2012]), our framework is capable of providing closed-form and tight bounds on the waiting time, while maintaining deterministic assumptions.

\section{Overview of the Chapter}

(a) In subsection 2.1, we introduce the notion of a robust queue as an alternative to the traditional queueing model and propose to replace the renewal process primitives with uncertainty sets that the arrival and service processes satisfy. 
(b) In subsection 2.2, we analyze the steady-state behavior of single and multi-server robust queues and obtain closed form expressions for the waiting times, which carry the same qualitative insights as traditional queueing theory and extend to include heavy-tailed arrivals and services.

(c) In subsection 2.3, we present an analog of Burke's theorem, where the uncertainty set characterizing the departure process is shown to be the same as the uncertainty set characterizing the arrival process. This is a remarkable property of our uncertainty set model which holds in considerable more generality than the $M / M / m$ queue.

(d) Armed with the analog of Burke's theorem, in subsection 2.4, we develop a calculus describing the effect of the three operations characterizing queueing networks on the arrival uncertainty set: arrival superposition, process thinning which models probabilistic routing, and passing through a queue. This allows us to exactly characterize the arrival process of any queue that operates in a queueing network.

(e) In subsection 2.5, we analyze queueing networks with asymmetric heavy-tailed arrival and service processes. In particular, we provide an extension of our results in subsections 2.2-2.4 to accommodate the case where arrival and service times possess different tail behaviors.

(f) In subsection 2.6, we report computational results for multi-server queueing networks, which suggest that the proposed approach can be adapted to be within 4-6\% from simulation. We also report on the sensitivity of the results as a function of the number of servers per queue, the network size, degree of feedback, traffic intensity, and the degree of diversity of external arrival distributions in the network. 


\subsection{The New Primitives}

We introduce the notion of a robust queue where we model the arrival and service processes by uncertainty sets instead of assigning probability distributions.

\subsubsection{The Robust Queue Model}

We denote the inter-arrival time between the $(i-1)^{s t}$ and $i^{t h}$ jobs by $T_{i}$ and the service time of job $i$ by $X_{i}$. We seek to analyze the worst case waiting time of the $n^{\text {th }}$ job which only depends on the services and inter-arrival times of the first $n$ jobs. In particular, we use the minimal inequalities required to analyze the worst case waiting time, namely the lower bounding constraints for the arrival process and upper bounding constraints for the service process. Therefore, we propose the following $n$-dimensional uncertainty sets on the arrival and service processes in Assumption 1.

Assumption 1. We make the following assumptions for the inter-arrival and service times

(a) The inter-arrival times $\left\{T_{1}, T_{2}, \ldots, T_{n}\right\}$ belong to the parametrized uncertainty set

$$
\mathcal{U}^{a}\left(\Gamma_{a}\right)=\left\{\left(T_{1}, T_{2}, \ldots, T_{n}\right) \mid \frac{\sum_{i=k+1}^{n} T_{i}-\frac{(n-k)}{\lambda}}{(n-k)^{1 / \alpha_{a}}} \geq-\Gamma_{a}, \forall 1 \leq k \leq n-1\right\}
$$

where $1 / \lambda$ is the expected inter-arrival time, $\Gamma_{a}$ is a parameter that captures variability information, and $1<\alpha_{a} \leq 2$ models possibly heavy-tailed probability distributions.

(b) The service times $\left\{X_{1}, X_{2}, \ldots, X_{n-1}\right\}$ for a single-server queue belong to the 
parametrized uncertainty set

$$
\mathcal{U}^{s}\left(\Gamma_{s}\right)=\left\{\left(X_{1}, X_{2}, \ldots, X_{n-1}\right) \mid \frac{\sum_{i=k}^{n-1} X_{i}-\frac{(n-k)}{\mu}}{(n-k)^{1 / \alpha_{s}}} \leq \Gamma_{s}, \forall 0 \leq k \leq n-1\right\}
$$

where $1 / \mu$ is the expected service time, $\Gamma_{s}$ is a parameter that captures variability information, and $1<\alpha_{s} \leq 2$ models possibly heavy-tailed probability distributions.

(c) For an $m$-server queue, we suppose $n=v m+r$ where $v$ and $r$ respectively denote the quotient and the remainder of the division of $n$ by $m$. The service times $\left\{X_{n-v m}, \ldots, X_{n-m}\right\}$ belong to the parametrized uncertainty set

$$
\mathcal{U}_{m}^{s}\left(\Gamma_{s}\right)=\left\{\left(X_{n-v m}, \ldots, X_{n-m}\right) \mid \frac{\sum_{i=k}^{v-1} X_{n-(v-i) m}-\frac{(v-k)}{\mu}}{(v-k)^{1 / \alpha_{s}}} \leq \Gamma_{s}, \forall k \leq v-1\right\}
$$

Note that we assume that both the inter-arrival and service times have bounded support as seen by letting $k=n-1$ in the sets $\mathcal{U}^{a}, \mathcal{U}_{m}^{s}$, and $\mathcal{U}^{s}$. The key idea in the construction of our uncertainty sets is to make the primitive assumptions follow from the major conclusions of probability theory, namely its asymptotic laws as opposed to the probability axioms. In the next section, we propose constructing the uncertainty sets based on the central limit theorem (CLT) and the stable limit laws.

\subsubsection{Construction of Uncertainty Sets}

We motivate the construction of these uncertainty sets through probabilistic weak convergence theorems. These theorems express the distribution of the sum of many independent and identically distributed random variables as converging to one of a small set of stable distributions. In particular, we use the Central Limit Theorem (CLT) to construct uncertainty sets for light-tailed distributions and Stable Limit 
Laws for heavy-tailed distributions.

\section{Light Tailed Distributions:}

Suppose that the inter-arrival and service times are independent and identically distributed with finite standard deviation $\sigma_{a}$ and $\sigma_{s}$, respectively. By the central limit theorem, as $n \rightarrow \infty$, the random variables

$$
\frac{\sum_{i=k+1}^{n} T_{i}-\frac{n-k}{\lambda}}{\sigma_{a}(n-k)^{1 / 2}} \text { and } \frac{\sum_{i=k+1}^{n} X_{i}-\frac{n-k}{\mu}}{\sigma_{s}(n-k)^{1 / 2}}
$$

are asymptotically standard normal. We know that a standard normal $Z$ satisfies $\mathbb{P}(Z \leq 2) \approx 0.975, \mathbb{P}(Z \leq 3) \approx 0.995$. We therefore assume that the quantities $T_{i}$ and $X_{i}$ take values such that

$$
\sum_{i=k+1}^{n} T_{i}-\frac{n-k}{\lambda} \geq-\Gamma_{a}(n-k)^{1 / 2} \quad \text { and } \quad \sum_{i=k+1}^{n} X_{i}-\frac{n-k}{\mu} \leq \Gamma_{s}(n-k)^{1 / 2}
$$

with $\Gamma_{a}$ and $\Gamma_{s}$ are variability parameters that can be adapted to ensure a good empirical fit (see Section 7 for computational results). Note that the tail coefficient implied by the CLT is $\alpha_{a}=\alpha_{s}=2$.

\section{Heavy Tailed Distributions:}

Under a probabilistic framework, a sequence of random variables $\left\{Y_{i}\right\}_{i \geq 1}$ heavy tailed distributions whose variance is undefined, are associated with heavy-tailed distributions. Such random variables satisfy the Stable Limit Laws as presented in Theorem 3 (see Nolan [1997]).

Theorem 3. (Nolan [1997]) Let $Y_{1}, Y_{2}, \ldots$ be a sequence of i.i.d. random variables, with mean $\mu$ and undefined variance. If $Y_{i} \sim Y$, where $Y$ is a stable distribution with parameter $\alpha \in(1,2]$ then

$$
\frac{\sum_{i=1}^{n} Y_{i}-n \mu}{n^{1 / \alpha}} \sim Y
$$


In our framework, the uncertain quantities we consider are bounded (see Assumption 1), but we use the specific $(n-k)^{1 / \alpha}$ scaling of Eq. (2.2) to motivate the construction of the uncertainty sets, and associate the term "heavy tails" with the coefficient $\alpha$ in the scaling. In other words, we use the conclusions of the the Stable Limit Laws of probability theory, and not its axioms and assumptions. Moreover, intuitively, by using a value of $\alpha_{a}, \alpha_{s}<2$, we allow the inter-arrival and service times to take larger values when compared to $\alpha_{a}, \alpha_{s}=2$, thus, allowing us to model the heavy-tailed nature.

We note that while the uncertainty sets are motivated by i.i.d. assumptions on the underlying random variables, $\left(T_{1}, T_{2}, \ldots, T_{n}\right) \in \mathcal{U}^{a}$ does not necessarily imply that $\left(T_{1}, T_{2}, \ldots, T_{n}\right)$ are independent. In summary, the key data primitives characterizing

(a) the arrival process in the queue are $\left(\lambda, \Gamma_{a}, \alpha_{a}\right)$;

(b) the service process in the queue are $\left(\mu, \Gamma_{s}, \alpha_{s}\right)$.

We first assume that arrival and service processes have symmetric tail behavior, i.e., $\alpha_{a}=\alpha_{s}=\alpha$ in subsections $2.3-2.5$, and then provide the generalized results for the asymmetric case in subsection 2.6.

\subsection{The Multi-Server Robust Queue}

In this section, we analyze the robust queue model with a first-come first-served scheduling policy and a traffic intensity $\rho=\lambda /(m \mu)<1$, where $m$ denotes the number of servers in the queue. We denote by $W_{n}$ the waiting time of the $n^{\text {th }}$ job in this system. Kingman $[1970]$ provides insightful bounds on the expected waiting time in steady state for the $G I / G I / 1$ queue

$$
\mathbb{E}\left[W_{n}\right] \leq \frac{\lambda}{2} \cdot \frac{\sigma_{a}^{2}+\sigma_{s}^{2}}{1-\rho}
$$


and for the $G I / G I / m$ queue

$$
\mathbb{E}\left[W_{n}\right] \leq \frac{\lambda}{2} \cdot \frac{\sigma_{a}^{2}+\sigma_{s}^{2} / m+\left(1 / m-1 / m^{2}\right) / \mu^{2}}{1-\rho}
$$

While $\mathbb{E}\left[W_{n}\right]$ seeks the expected waiting time when nature obeys the axioms of probability, we seek the highest waiting time when nature is constrained to obey the limit laws of probability. Assuming inter-arrival times $\left\{T_{i}\right\}_{i \geq 1} \in \mathcal{U}^{a}$ and service times $\left\{X_{i}\right\}_{i \geq 1} \in \mathcal{U}_{m}^{s}$, we define the highest waiting time

$$
\widehat{W}_{n}=\max _{\mathbf{T} \in \mathcal{U}^{a}, \mathbf{X} \in \mathcal{U}_{m}^{s}} W_{n}
$$

and the steady-state worst-case waiting time as

$$
\widehat{W}=\lim _{n \rightarrow \infty} \widehat{W}_{n}
$$

We characterize $\widehat{W}$ for robust queues with single and multiple servers for heavy-tailed arrival and service processes.

\subsubsection{Waiting Time in a Single-Server Robust Queue}

We consider a robust queue with a single server and provide a closed form expression for $\widehat{W}$.

Theorem 4. If $\left\{T_{i}\right\}_{i \geq 1} \in \mathcal{U}^{a},\left\{X_{i}\right\}_{i \geq 1} \in \mathcal{U}^{s}, \alpha_{a}=\alpha_{s}=\alpha$ and $\rho<1$, then

$$
\widehat{W} \leq \frac{\alpha-1}{\alpha^{\alpha /(\alpha-1)}} \cdot \frac{\lambda^{1 /(1-\alpha)}\left(\Gamma_{a}+\Gamma_{s}\right)^{\alpha /(\alpha-1)}}{(1-\rho)^{1 /(\alpha-1)}}
$$

Proof. The waiting time of the $n^{\text {th }}$ job can be expressed recursively in terms of the inter-arrival and service times using the Lindley recursion (Lindley [1952])

$$
W_{n}=\max \left(W_{n-1}+X_{n-1}-T_{n}, 0\right)=\max _{1 \leq j \leq n-1}\left(\sum_{\ell=j}^{n-1} X_{\ell}-\sum_{\ell=j+1}^{n} T_{\ell}, 0\right)
$$


Thus, $\widehat{W}_{n}$ can be written as

$$
\begin{aligned}
\widehat{W}_{n} & =\max _{\mathbf{X} \in \mathcal{U}^{s}, \mathbf{T} \in \mathcal{U}^{a}} \max _{1 \leq j \leq n-1}\left(\sum_{\ell=j}^{n-1} X_{\ell}-\sum_{\ell=j+1}^{n} T_{\ell}, 0\right) \\
& =\max _{1 \leq j \leq n-1} \max _{\mathbf{x} \in \mathcal{U}^{s}, \mathbf{T} \in \mathcal{U}^{a}}\left(\sum_{\ell=j}^{n-1} X_{\ell}-\sum_{\ell=j+1}^{n} T_{\ell}, 0\right)
\end{aligned}
$$

From Assumption 1, for $j \leq n-1$, the sums of the service times and inter-arrival times are bounded by

$$
\sum_{\ell=j}^{n-1} X_{\ell} \leq \frac{n-j}{\mu}+\Gamma_{s}(n-j)^{1 / \alpha}, \sum_{\ell=j+1}^{n} T_{\ell} \geq \frac{n-j}{\lambda}-\Gamma_{a}(n-j)^{1 / \alpha}
$$

Combining Eqs. (2.9) and (2.10), we obtain an one-dimensional concave maximization problem (since $1<\alpha \leq 2$ )

$$
\max _{1 \leq j \leq n-1} \max _{\mathbf{X} \in \mathcal{U}^{s}, \mathbf{T} \in \mathcal{U}^{a}} \sum_{\ell=j}^{n-1} X_{\ell}-\sum_{\ell=j+1}^{n} T_{\ell} \leq \max _{1 \leq j \leq n-1}\left\{\left(\Gamma_{a}+\Gamma_{s}\right)(n-j)^{1 / \alpha}-\frac{1-\rho}{\lambda}(n-j)\right\} .
$$

Making the transformation $x=n-j$, Eq. (2.11) becomes

$$
\max _{1 \leq j \leq n-1} \max _{\mathbf{X} \in \mathcal{U}^{s}, \mathbf{T} \in \mathcal{U}^{a}} \sum_{\ell=j}^{n-1} X_{\ell}-\sum_{\ell=j+1}^{n} T_{\ell} \leq \max _{1 \leq x \leq n-1} \beta \cdot x^{1 / \alpha}-\gamma \cdot x
$$

with $\beta=\Gamma_{a}+\Gamma_{s}$ and $\gamma=(1-\rho) / \lambda>0$, given $\rho<1$. Hence,

$$
\max _{1 \leq j \leq n-1} \max _{\mathbf{X} \in \mathcal{U}^{s}, \mathbf{T} \in \mathcal{U}^{a}} \sum_{\ell=j}^{n-1} X_{\ell}-\sum_{\ell=j+1}^{n} T_{\ell} \leq \max _{1 \leq x \leq n-1} \beta \cdot x^{1 / \alpha}-\gamma \cdot x \leq \max _{x \geq 0} \beta \cdot x^{1 / \alpha}-\gamma \cdot x
$$

The maximizer in Eq. (2.13) is given by

$$
x^{*}=\left(\frac{\beta}{\alpha \gamma}\right)^{\alpha /(\alpha-1)}=\left(\frac{\lambda\left(\Gamma_{a}+\Gamma_{s}\right)}{\alpha(1-\rho)}\right)^{\alpha /(\alpha-1)} .
$$


Substituting $\beta$ and $\gamma$ by their respective expressions in Eq. (2.13), we obtain

$$
\max _{1 \leq j \leq n-1} \max _{\mathbf{x} \in \mathcal{U}^{s}, \mathbf{T} \in \mathcal{U}^{a}} \sum_{\ell=j}^{n-1} X_{\ell}-\sum_{\ell=j+1}^{n} T_{\ell} \leq \frac{\alpha-1}{\alpha^{\alpha /(\alpha-1)}} \cdot \frac{\lambda^{1 /(1-\alpha)}\left(\Gamma_{a}+\Gamma_{s}\right)^{\alpha /(\alpha-1)}}{(1-\rho)^{1 /(\alpha-1)}}
$$

\section{Remark on Theorem 4 - Tightness of the bound}

We note that the upper bound on the waiting time in Theorem 4 is nearly tight. Let us first consider the case where the maximizer $x^{*}$ in Eq. (2.14) is an integer, and let $j^{*}=n-x^{*}$. The following sequence of inter-arrival times

$$
T_{i}= \begin{cases}1 / \lambda & i=1, \ldots, j^{*} \\ 1 / \lambda-\Gamma_{a} /\left(x^{*}\right)^{1-1 / \alpha} & i=j^{*}+1, \ldots, n\end{cases}
$$

and service times

$$
X_{i}= \begin{cases}1 / \mu & i=1, \ldots, j^{*}-1 \\ 1 / \mu+\Gamma_{s} /\left(x^{*}\right)^{1-1 / \alpha} & i=j^{*}, \ldots, n-1\end{cases}
$$

lie in the uncertainty sets in Assumption 1. This can be seen via the partial sums given by

$$
\sum_{i=j+1}^{n} T_{i}= \begin{cases}(n-j) / \lambda-\Gamma_{a}\left(x^{*}\right)^{1 / \alpha} & j=1, \ldots, j^{*}-1 \\ (n-j) / \lambda-\Gamma_{a}(n-j) /\left(x^{*}\right)^{1-1 / \alpha} & j=j^{*}, \ldots, n-1\end{cases}
$$

and

$$
\sum_{i=j}^{n-1} X_{i}= \begin{cases}(n-j) / \mu+\Gamma_{s}\left(x^{*}\right)^{1 / \alpha} & j=1, \ldots, j^{*}-1, \\ (n-j) / \mu+\Gamma_{s}(n-j) /\left(x^{*}\right)^{1-1 / \alpha} & j=j^{*}, \ldots, n-1,\end{cases}
$$


and noting that $(n-j) /\left(x^{*}\right)^{1-1 / \alpha} \leq(n-j)^{1 / \alpha}$ for all $j=j^{*}, \ldots, n-1$. We then obtain

$$
\sum_{i=j}^{n-1} X_{i}-\sum_{i=j+1}^{n} T_{i}= \begin{cases}\left(\Gamma_{a}+\Gamma_{s}\right)\left(x^{*}\right)^{1 / \alpha}-(1-\rho)(n-j) / \lambda & j<j^{*} \\ \left(\Gamma_{a}+\Gamma_{s}\right)(n-j) /\left(x^{*}\right)^{1-1 / \alpha}-(1-\rho)(n-j) / \lambda & j \geq j^{*}\end{cases}
$$

Note that, since $\left(\Gamma_{a}+\Gamma_{s}\right)\left(x^{*}\right)^{1 / x}$ is a constant with respect to $j$,

$$
\begin{array}{r}
\max _{1 \leq j \leq j^{*}-1}\left\{\left(\Gamma_{a}+\Gamma_{s}\right)\left(x^{*}\right)^{1 / \alpha}-(1-\rho)(n-j) / \lambda\right\} \\
<\left(\Gamma_{a}+\Gamma_{s}\right)\left(x^{*}\right)^{1 / \alpha}-(1-\rho)\left(x^{*}\right) / \lambda \\
=\frac{\alpha-1}{\alpha^{\alpha /(\alpha-1)}} \cdot \frac{\lambda^{1 /(1-\alpha)}\left(\Gamma_{a}+\Gamma_{s}\right)^{\alpha /(\alpha-1)}}{(1-\rho)^{1 /(\alpha-1)}}
\end{array}
$$

The first strict inequality is due to the fact that for $j \leq j^{*}-1=n-x^{*}-1$, we have $x^{*}<n-j$. Moreover, for $j \geq j^{*}$, we have $n-j \leq n-j^{*}$, and given that $x^{*}$ is the maximizer of Eq. (2.5), we have

$$
\begin{array}{r}
\left(\Gamma_{a}+\Gamma_{s}\right)(n-j) /\left(x^{*}\right)^{1-1 / \alpha}-(1-\rho)(n-j) / \lambda \\
\leq\left(\Gamma_{a}+\Gamma_{s}\right)(n-j)^{1 / \alpha}-(1-\rho)(n-j) / \lambda \\
\leq\left(\Gamma_{a}+\Gamma_{s}\right)\left(x^{*}\right)^{1 / \alpha}-(1-\rho)\left(x^{*}\right) / \lambda
\end{array}
$$

Therefore, we have shown that when the maximizer $x^{*}$ is an integer, then there exists a sequence of inter-arrival and service times that exactly achieves a waiting time equal to the bound in Eq. (2.7). We next show that when the maximizer $x^{*}$ is fractional, then the actual highest waiting time is within a fraction $\mathcal{O}\left((1-\rho)^{\alpha /(\alpha-1)}\right)$ to the bound in Eq. (2.7). Note that when $x^{*}$ is fractional, due to the concave nature of the function in Eq. (2.13), the optimal value $j^{*}$ of Eq. (2.11) is either $n-\left\lceil x^{*}\right\rceil$ or $n-\left\lfloor x^{*}\right\rfloor$. Denoting the function in Eq. (2.13) by $f(x)=\beta x^{1 / \alpha}-\gamma x$ with $f\left(x^{*}\right)$ given 
by Eq. (2.7), we obtain

$$
\begin{aligned}
0 & \geq \frac{f\left(\left[x^{*}\right]\right)-f\left(x^{*}\right)}{f\left(x^{*}\right)} \\
& \geq \frac{f\left(x^{*}+1\right)-f\left(x^{*}\right)}{f\left(x^{*}\right)} \\
& =\frac{1}{f\left(x^{*}\right)} \cdot\left\{\beta \cdot\left\{\left(x^{*}+1\right)^{1 / \alpha}-\left(x^{*}\right)^{1 / \alpha}\right\}-\gamma\right\} \\
& \geq \frac{\alpha^{\alpha /(\alpha-1)} \gamma^{1 /(\alpha-1)}}{\beta^{\alpha /(\alpha-1)}} \cdot\left\{\beta \cdot \frac{1}{\left.\alpha\left(x^{*}+1\right)^{1-1 / \alpha}-\gamma\right\}}\right. \\
& \geq \frac{\alpha^{\alpha /(\alpha-1)} \gamma^{1 /(\alpha-1)}}{\beta^{\alpha /(\alpha-1)}} \cdot\left\{\frac{\beta}{\alpha} \cdot \frac{1}{\left(1+(\beta / \alpha \gamma)^{\alpha /(\alpha-1)}\right)^{1-1 / \alpha}}-\gamma\right\} \\
& =\frac{\alpha^{\alpha /(\alpha-1)} \gamma^{1 /(\alpha-1)}}{\beta^{\alpha /(\alpha-1)}} \cdot\left\{\frac{\beta}{\alpha} \cdot \frac{\alpha \gamma}{\left((\alpha \gamma)^{\alpha /(\alpha-1)}+\beta^{\alpha /(\alpha-1)}\right)^{1-1 / \alpha}}-\gamma\right\} \\
& \geq \frac{\alpha^{\alpha /(\alpha-1)} \gamma^{1 /(\alpha-1)}}{\beta^{\alpha /(\alpha-1)}} \cdot\left\{\frac{\beta}{\alpha} \cdot \frac{\alpha \gamma}{\alpha \gamma+\beta}-\gamma\right\} \\
& =\frac{-\alpha^{(2 \alpha-1) /(\alpha-1)} \gamma^{\alpha /(\alpha-1)}}{\beta^{\alpha /(\alpha-1)}(\beta+\alpha \gamma)}=-\mathcal{O}\left((1-\rho)^{\alpha /(\alpha-1)}\right) .
\end{aligned}
$$

Eq. (2.20) follows from Eq. (2.19) by the concavity of $g(x)=x^{1 / \alpha}$ function. Eq. (2.22) follows from Eq. (2.21) given that $(a+b)^{1-1 / \alpha} \leq a^{1-1 / \alpha}+b^{1-1 / \alpha}$. In the same way we can bound the quantity $\left(f\left(\left\lfloor x^{*}\right\rfloor\right)-f\left(x^{*}\right)\right) / f\left(x^{*}\right)$. In summary, we have shown that the bounds presented in Theorem 4 are nearly tight.

\subsubsection{Waiting Time in a Multi-Server Robust Queue}

We consider a queue with $m$ parallel servers and denote by $A_{n}$ the arrival time of the $n^{\text {th }}$ job where $A_{n}=\sum_{\ell=1}^{n} T_{\ell}$ for every $n$, and $C_{n}$ the completion time of the $n^{\text {th }}$ job, i.e., the time the $n^{\text {th }}$ job leaves the system (including service). The central difficulty in analyzing probabilistic multi-server queues lies in the fact that overtaking may occur, i.e., the $n^{\text {th }}$ departing job is not necessarily the $n^{\text {th }}$ arriving job. To address this matter, we introduce the ordered sequence of completion times $C_{(1)} \leq C_{(2)} \leq \ldots \leq C_{(n)}$ and define $D_{n}$ as the $n^{\text {th }}$ interdeparture time given by $D_{n}=C_{(n)}-C_{(n-1)}$. We briefly review the dynamics of the multi-server queuc. Figure 2-1 depicts a two-server queue and the associated quantities of interest $A_{n}$, 
$X_{n}, W_{n}, C_{n}, C_{(n)}$, and $D_{n}$.

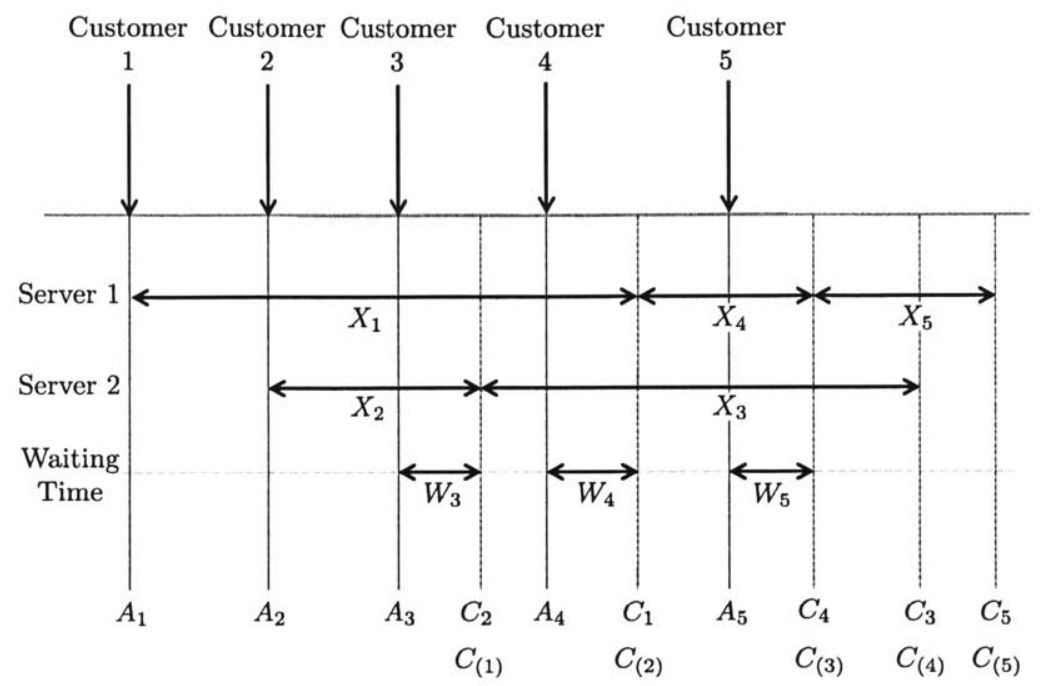

Figure 2-1: Dynamics of a Two-Sever Queue.

Looking at the snapshot of the process for five jobs, the waiting times can be found as

$$
\begin{gathered}
W_{1}=0, W_{2}=0, \quad W_{3}=C_{2}-A_{3}=C_{(1)}-A_{3}, \\
W_{4}=C_{1}-A_{4}=C_{(2)}-A_{4}, \quad W_{5}=C_{4}-A_{5}=C_{(3)}-A_{5} .
\end{gathered}
$$

By induction, we obtain the general expression of the $n^{\text {th }}$ waiting time

$$
W_{n}=\max \left\{C_{(n-m)}-A_{n}, 0\right\}
$$

as is well established (see Kiefer and Wolfowitz [1955]). Notice that Eq. (2.23) generalizes the Lindley recursion to the multi-server case. Furthermore, we note that

$$
\begin{aligned}
& C_{n}=A_{n}+W_{n}+X_{n}=A_{n}+S_{n}, \\
& C_{0}=0 \text { and } C_{r}=A_{r}+X_{r} \text { for } 1 \leq r \leq m,
\end{aligned}
$$

where $S_{n}=W_{n}+X_{n}$ denotes the sojourn time of the $n^{\text {th }}$ job. Note that Eq. (2.25) 
follows by the fact that the first $m$ jobs do not wait. We now provide a closed form expression for $\widehat{W}$.

Theorem 5. If $\left\{T_{i}\right\}_{i \geq 1} \in \mathcal{U}^{a},\left\{X_{i}\right\}_{i \geq 1} \in \mathcal{U}_{m}^{s}, \alpha_{a}=\alpha_{s}=\alpha$ and $\rho<1$, then

$$
\widehat{W} \leq \frac{\alpha-1}{\alpha^{\alpha /(\alpha-1)}} \cdot \frac{\lambda^{1 /(\alpha-1)}\left(\Gamma_{a}+\Gamma_{s} / m^{1 / \alpha}\right)^{\alpha /(\alpha-1)}}{(1-\rho)^{1 /(\alpha-1)}} .
$$

Proof. Our proof proceeds in two steps: we first analyze our system by considering a scheduling policy that effectively treats the multi-server system as parallel single servers and leads to a policy where no overtaking occurs. This allows us to obtain an upper bound on the worst case waiting time; we then show that this upper bound is indeed achieved by a sample path that leads to no overtaking which shows that the upper bound is tight. Therefore, let us consider a system where the scheduling policy does not lead to any overtaking, and consequently leads to an upper bound on the waiting time. For such a system, we have $C_{(i)}=C_{i}$, and therefore by combining Eqs.

$$
\begin{aligned}
C_{(n-m)} & \leq \max \left\{C_{(n-2 m)}, A_{n-m}\right\}+X_{n-m} \\
& \leq \max \left\{\max \left\{C_{(n-3 m)}, A_{n-2 m}\right\}+X_{n-2 m}, A_{n-m}\right\}+X_{n-m}, \\
& \leq \max \left\{C_{(n-3 m)}+X_{n-2 m}+X_{n-m}, A_{n-2 m}+X_{n-2 m}+X_{n-m}, A_{n-m}+X_{n-m}\right\} .
\end{aligned}
$$

Let $n=v m+r, 0 \leq r<m$, where $r$ is the remainder of the division of $n$ by $m$. Thus,

$$
C_{(n-m)} \leq \max \left\{C_{(n-v m)}+\sum_{k=1}^{v-1} X_{n-k m}, A_{n-(v-1) m}+\sum_{k=1}^{v-1} X_{n-k m}, \ldots, A_{n-m}+X_{n-m}\right\}
$$

From Eq. (2.23), the $n^{\text {th }}$ waiting time is as follows

$$
W_{n} \leq \max \left\{C_{(n-v m)}+\sum_{k=1}^{v-1} X_{n-k m}-A_{n}, A_{n-(v-1) m}+\sum_{k=1}^{v-1} X_{n-k m}-A_{n}, \ldots, 0\right\}
$$

Note that $n-v m=r$ and $W_{r}=0$ yielding $C_{(r)} \leq C_{r}=A_{r}+X_{r}$, for all $0 \leq r<m$. 
Then,

$$
W_{n} \leq \max \left\{A_{r}+X_{r}+\sum_{k=1}^{v-1} X_{(v-k) m+r}-A_{n}, A_{m+r}+\sum_{k=1}^{v-1} X_{(v-k) \cdot m+r}-A_{n}, \ldots, 0\right\}
$$

Expressing the arrival times as $A_{n}=\sum_{\ell=1}^{n} T_{\ell}$ for every $n$, we obtain

$$
W_{n} \leq \max \left\{\sum_{k=1}^{v} X_{(v-k) m+r}-\sum_{\ell=r+1}^{n} T_{\ell}, \sum_{k=1}^{v-1} X_{(v-k) m+r}-\sum_{\ell=m+r+1}^{n} T_{\ell}, \ldots, 0\right\}
$$

By substituting $\ell=v-k$, the above expression can be re-written as

$$
W_{n} \leq \max _{0 \leq j \leq v-1}\left\{\sum_{\ell=j}^{v-1} X_{\ell m+r}-\sum_{\ell=j m+r+1}^{v m+r} T_{\ell}, 0\right\}
$$

Note that if we let $m=1$ in Eq. (2.27), we recover Eq. (2.8) for the single-server case.

Moreover, since $\left\{X_{i}\right\}_{i \geq 1} \in \mathcal{U}_{m}^{s}$ and $\left\{T_{i}\right\}_{i \geq 1} \in \mathcal{U}^{a}$

$$
\sum_{\ell=j}^{v-1} X_{\ell m+r} \leq \frac{v-j}{\mu}+\Gamma_{s}(v-j)^{1 / \alpha}, \sum_{\ell=j m+r+1}^{v m+r} T_{\ell} \geq \frac{m(v-j)}{\lambda}-m^{1 / \alpha} \Gamma_{a}(v-j)^{1 / \alpha},
$$

we obtain

$$
\widehat{W}_{n} \leq \max _{0 \leq j \leq v-1}\left\{\left(m^{1 / \alpha} \Gamma_{a}+\Gamma_{s}\right)(v-j)^{1 / \alpha}-\frac{m(1-\rho)}{\lambda}(v-j)\right\}
$$

Making the change of variables $x=v-j$, we obtain that

$$
\widehat{W}_{n} \leq \max _{1 \leq x \leq v}\left\{\left(m^{1 / \alpha} \Gamma_{a}+\Gamma_{s}\right) x^{1 / \alpha}-\frac{m(1-\rho)}{\lambda} x\right\}
$$

leading to

$$
\widehat{W} \leq \max _{1 \leq x}\left\{\left(m^{1 / \alpha} \Gamma_{a}+\Gamma_{s}\right) x^{1 / \alpha}-\frac{m(1-\rho)}{\lambda} x\right\}
$$

Note that the right-hand side is an one dimensional concave maximization problem in the form of Eq. (2.13), maximized at $x^{*}$ (refer to Eq. (2.14)), with $\beta=m^{1 / \alpha} \Gamma_{a}+\Gamma_{s}$ 
and $\gamma=m(1-\rho) / \lambda>0$, given $\rho<1$. Substituting $\beta$ and $\gamma$ by their respective values in Eq. (2.13) yields Eq. (2.26).

We next present a sequence of inter-arrival times that exactly achieves a waiting time equal to the bound in Eq. (2.26). Consider the following sequence of interarrival and service times, and assume $x^{*}$ is an integer given by $x^{*}=m \bar{v}+\bar{r}$ and let $j^{*}=v-\bar{v}$

$$
\begin{gathered}
T_{i}= \begin{cases}1 / \lambda & i=1, \ldots, m j^{*}+r, \\
\frac{1}{\lambda}-\frac{\Gamma_{a}}{\left(x^{*}\right)^{(\alpha-1) / \alpha}} & i=m j^{*}+r+1, \ldots, n,\end{cases} \\
X_{i m+r}= \begin{cases}1 / \mu & i=1, \ldots, j^{*}-1, \\
\frac{1}{\mu}+\frac{\Gamma_{s}}{\bar{v}^{(\alpha-1) / \alpha}} & i=j^{*}, \ldots, v-1,\end{cases}
\end{gathered}
$$

and choose $X_{k}=1 / \mu$ for all other jobs. As before, these sequences belong to $\mathcal{U}^{a}$ and $\mathcal{U}_{m}^{s}$, respectively and also do not allow any overtaking. To see that they do not allow overtaking, note that a later job always experiences a worse service time and an early inter-arrival time leading to more waiting time. This concludes the proof.

\subsubsection{Implications and Insights}

To summarize, we obtain the following characterization of the waiting time in an $m$-server queue

$$
\widehat{W} \leq \frac{\alpha-1}{\alpha^{\alpha /(\alpha-1)}} \cdot \frac{\lambda^{1 /(\alpha-1)}\left(\Gamma_{a}+\Gamma_{s} / m^{1 / \alpha}\right)^{\alpha /(\alpha-1)}}{(1-\rho)^{1 /(\alpha-1)}}
$$

where the expression simplifies to Eq. (2.7) for $m=1$. We present next the main implications and insights that follow from this closed form expression.

(a) Qualitative Insights 
The robust queue behaves qualitatively the same as the traditional queue. For instance, the classical i.i.d. arrival and service processes with finite variance can be modeled by setting $\alpha=2$. For the single server queue, Eq. (2.31) becomes

$$
\widehat{W}_{n}=\frac{\lambda}{4} \cdot \frac{\left(\Gamma_{a}+\Gamma_{s}\right)^{2}}{(1-\rho)}
$$

and for the multi-server queue

$$
\widehat{W}_{n}=\frac{\lambda}{4} \cdot \frac{\left(\Gamma_{a}+\Gamma_{s} / m^{1 / 2}\right)^{2}}{1-\rho}
$$

Contrasting Kingman's bounds (2.3) and (2.4) with the bounds (2.32) and (2.33), we observe that they have the same functional dependence on $\lambda /(1-\rho)$ and on the variability parameters $\Gamma_{a}^{2}, \Gamma_{s}^{2} / m$, (correspondingly $\sigma_{a}^{2}, \sigma_{s}^{2} / m$ ). In this sense, our approach leads to the same qualitative insights as stochastic queueing theory.

\section{(b) Modeling Heavy-Tailed Behavior}

Our approach allows a closed-form expression for the steady-state waiting time for all values of $\alpha \in(1,2)$, which include heavy tailed random variables. Observe that heavier the tail, i.e., the smaller the tail coefficient $\alpha$, the higher the order of the waiting time, given its dependence on $1 /(1-\rho)^{1 /(\alpha-1)}$. To illustrate, a decrease in the tail coefficient from $\alpha=2$ to $\alpha=1.5$ increases the waiting time by one order of magnitude. This is in agreement with the stochastic queueing theory literature, where it is known that the waiting time exhibits a heavy-tailed distribution under heavy tailed services (see Whitt [2000], Crovella [2000]).

During the past decade, studies have shown the existence of heavy-tailed behavior in some of the main application areas of queueing theory: (a) Call centers (Barabasi [2005]), (b) Data centers and cloud computing (Loboz [2012], Benson et al. [2010]), and (c) Internet (Willinger et al. [1998], Leland et al. [1995], Crovella [1997], Jelenkovic et al. [1997], Kumar et al. [2000]). Our closed form results allow practioners in these application domains to understand the dependence of waiting times on various system parameters such as the number of servers $m$ and traffic intensity $\rho$. 


\subsection{Departure Process of Robust Queues}

Understanding the mechanism of the departure process from a queue is central to analyzing networks of queues. As we discussed in Section 1, renewal arrivals do not lead to renewal departures with the exception of the $M / M / m$ queue. In contrast, in our approach, we obtain a characterization of the departure process and show that it enables network analysis in the same way Burke's theorem enables network analysis for $M / M / 1$ queues.

\subsubsection{Departure Process in a Single-Sever Robust Queue}

We consider the single-sever robust queue and seek to characterize the uncertainty set of the departure process. In Theorem 6 , we show that the uncertainty set $\mathcal{U}^{d}$ that characterizes the sequence of the departure times $\left\{D_{i}\right\}_{i=1}^{n}$ is a subset of the arrival process $\mathcal{U}_{a}$.

Theorem 6. Let the inter-arrival times $\left\{T_{i}\right\}_{i \geq 1} \in \mathcal{U}^{a}$, and services $\left\{X_{i}\right\}_{i \geq 1} \in \mathcal{U}^{s}$, with $\alpha_{a}=\alpha_{s}=\alpha$ and $\rho<1$. Then the sequence of subsequent inter-departure times $\left\{D_{i}\right\}_{i=1}^{n}$ belong to the uncertainty set $\mathcal{U}^{d}$ such that

$$
\mathcal{U}^{d} \subseteq\left\{\left(D_{1}, D_{2}, \ldots, D_{n}\right) \mid \frac{\sum_{i=k+1}^{n} D_{i}-\frac{n-k}{\lambda}}{(n-k)^{1 / \alpha}} \geq-\Gamma_{a}, \forall k \leq n-1\right\}
$$

Proof. We first consider the sum of the the inter-departure times from job 1 to job $n$. We have

$$
\begin{aligned}
\sum_{i=1}^{n} D_{i} & =W_{n}+X_{n}+\sum_{i=1}^{n} T_{i} \\
& =\max _{1 \leq j \leq n}\left(\sum_{i=j}^{n} X_{i}-\sum_{i=j+1}^{n} T_{i}\right)+\sum_{i=1}^{n} T_{i} \\
& =\max _{1 \leq j \leq n}\left(\sum_{i=j}^{n} X_{i}+\sum_{i=1}^{j} T_{i}\right)
\end{aligned}
$$


Next note that, from the perspective of the job $k+1$, the following two cases arise:

Case 1 . When the job $k+1$ sees an empty queue, that is $W_{k+1}=0$. In this case by using Eq. (2.35), we have

$$
\sum_{i=k+1}^{n} D_{i}=\max _{k+1 \leq j \leq n}\left(\sum_{i=j}^{n} X_{i}+\sum_{i=k+1}^{j} T_{i}\right)
$$

Case 2. When the job $k+1$ sees a non-empty queue, that is $W_{k+1}>0$. In this case, the job $k+1$ waits for a time $W_{k+1}$ thus delaying the departure of all subsequent jobs. Therefore, by again using Eq. (2.35), we obtain an inequality as opposed to an equality given by

$$
\sum_{i=k+1}^{n} D_{i} \geq \max _{k+1 \leq j \leq n}\left(\sum_{i=j}^{n} X_{i}+\sum_{i=k+1}^{j} T_{i}\right) .
$$

By combining Eqns. (2.36) and (2.37), we have

$$
\begin{aligned}
\sum_{i=k+1}^{n} D_{i} & \geq \max _{k+1 \leq j \leq n}\left(\sum_{i=j}^{n} X_{i}+\sum_{i=k+1}^{j} T_{i}\right) \\
& \geq X_{n}+\sum_{i=k+1}^{n} T_{i} \\
& \geq(n-k) / \lambda-\Gamma_{a} \cdot(n-k)^{1 / \alpha}
\end{aligned}
$$

This completes the proof.

\subsubsection{Departure Process in a Multi-Sever Robust Queue}

We generalize the characterization of the departure process uncertainty set to the multi-server robust queue.

Theorem 7. For an m-server queue, if $\left\{T_{i}\right\}_{i \geq 1} \in \mathcal{U}^{a},\left\{X_{i}\right\}_{i \geq 1} \in \mathcal{U}_{m}^{s}, \alpha_{a}=\alpha_{s}=\alpha$ and $\rho<1$, then the interdeparture times $\left\{D_{i}\right\}_{i \geq 1}$ belong to the uncertainty set $\mathcal{U}^{d}$ 
where

$$
\mathcal{U}^{d} \subseteq\left\{\left(D_{1}, D_{2}, \ldots, D_{n}\right) \mid \frac{\sum_{i=k+1}^{n} D_{i}-\frac{n-k}{\lambda}}{(n-k)^{1 / \alpha}} \geq-\Gamma_{a}, \forall k \leq n-1\right\}
$$

Proof. The proof proceeds in a manner very similar to that of the single server case. We first consider the sum of the the inter-departure times from job 1 to job $n$. Letting $n=\nu m+r$, where $r$ is the remainder we obtain by dividing $n$ by $m$, we have

$$
\begin{aligned}
\sum_{i=1}^{n} D_{i} & =W_{n}+X_{n}+\sum_{i=1}^{n} T_{i} \\
& \geq \max _{1 \leq j \leq n u}\left(\sum_{i=j}^{\nu} X_{i m+r}-\sum_{i=j m+r+1}^{\nu m+r} T_{i}\right)+\sum_{i=1}^{\nu m+r} T_{i} \\
& =\max _{1 \leq j \leq \nu}\left(\sum_{i=j}^{\nu} X_{i}+\sum_{i=1}^{j m+r} T_{i}\right)
\end{aligned}
$$

From the perspective of the job $k+1$ again, the following two cases arise:

Case 1. When the job $k+1$ sees an empty queue, that is $W_{k+1}=0$. In this case by using Eq. (2.39), we have

$$
\sum_{i=k+1}^{n} D_{i}=\max _{\lceil k+1 / n\rceil \leq j \leq \nu}\left(\sum_{i=j}^{\nu} X_{i}+\sum_{i=k+1}^{j m+r} T_{i}\right)
$$

Case 2. When the job $k+1$ sees a non-empty queue, that is $W_{k+1}>0$. In this case, the job $k+1$ waits for a time $W_{k+1}$ thus delaying the departure of all subsequent jobs. Therefore, by again using Eq. (2.39), we obtain an inequality as opposed to an equality given by

$$
\sum_{i=k+1}^{n} D_{i} \geq \max _{[k+1 / n] \leq j \leq \nu}\left(\sum_{i=j}^{\nu} X_{i}+\sum_{i=k+1}^{j m+r} T_{i}\right)
$$


By combining Eqns. (2.40) and (2.41), we have

$$
\begin{aligned}
\sum_{i=k+1}^{n} D_{i} & \geq \max _{\lceil k+1 / n\rceil \leq j \leq \nu}\left(\sum_{i=j}^{\nu} X_{i}+\sum_{i=k+1}^{j m+r} T_{i}\right) \\
& \geq X_{\nu}+\sum_{i=k+1}^{\nu m+r} T_{i} \\
& \geq \sum_{i=k+1}^{n} T_{i} \\
& \geq(n-k) / \lambda-\Gamma_{a} \cdot(n-k)^{1 / \alpha} .
\end{aligned}
$$

This completes the proof.

\subsubsection{Implications and Insights}

To summarize, we obtain that the uncertainty set describing the departure process from a multi-server queue is a subset of the arrival process, which implies that the worst case waiting time does not amplify as one proceeds through a network. We next discuss the implications and insights that follow from this result.

(a) Robust Burke's Theorem: Note that the departure process lies in a uncertainty set which is a subset of the input arrival process. This result allows us to extend our results on the waiting time $\mathrm{n}$ a network setting in the same way Burke's theorem from the stochastic queueing theory literature allows network analysis. In particular, we propose an algorithm in Section 2.6 which to perform network analysis.

(b) While the construction of the arrival uncertainty set using limit laws is motivated by iid inter-arrival times, membership in $\mathcal{U}^{a}$ does not imply that the interarrival times are independent. Similarly, membership in $\mathcal{U}^{d}$ does not imply that $\left\{D_{1}, \ldots, D_{n}\right\}$ are independent. This is consistent with stochastic queueing theory, where the interdeparture times are in general dependent. Our framework provides a concrete way to bound the departure process. 


\subsection{The Robust Queueing Network Analyzer}

Consider a network of $J$ queues serving a single class of jobs. Each job enters the network through some queue $j$, and either leaves the network or departs towards another queue right after completion of his service. The primitive data in the queueing network are:

(a) External arrival processes with parameters $\left(\lambda_{j}, \Gamma_{a, j}, \alpha_{a, j}\right)$ that arrive to each node $j=1, \ldots, J$.

(b) Service processes with parameters $\left(\mu_{j}, \Gamma_{s, j}, \alpha_{s, j}\right)$, and the number of servers $m_{j}, j=1, \ldots, J$.

(c) Routing matrix $\mathbf{F}=\left[f_{i j}\right], i, j=1, \ldots, J$, where $f_{i j}$ denotes the fraction of jobs passing through queue $i$ and are routed to queue $j$. The fraction of jobs leaving the network from queue $i$ is $1-\sum_{j} f_{i j}$.

In order to analyze the waiting time in a particular queue $j$ in the network, we need to characterize the overall arrival process to queue $j$ and then apply Theorem 4 for single-server and Theorem 5 for multi-server queues. The arrival process in queue $j$ is the superposition of different processes, each of which is either a process from the outside world, or a departure process from another queue, or a thinning of a departure process from another queue, or a thinning of an external arrival process. Correspondingly, in order to analyze the network, we need to characterize the effect that the following operations have on the arrival process:

(a) Passing through a queue: Under this operation, we characterize the departure process $\left\{D_{i}\right\}_{i \geq 1}$ when an arrival process $\left\{T_{i}\right\}_{i \geq 1} \in \mathcal{U}^{a}$ passes through a queue. We have already accomplished this in Theorems 6 and 7 for the single-server and multi-server queue, respectively, and have shown that $\left\{D_{i}\right\}_{i \geq 1} \in \mathcal{U}^{a}$ when $n \rightarrow \infty$.

(b) Superposition of arrival processes: Under this operation, $m$ arrival processes $\left\{T_{i}^{j}\right\}_{i \geq 1} \in \mathcal{U}_{j}^{a}, j=1, \ldots, m$ combine to form a single arrival process. Theorem 8 characterizes the uncertainty set of the combined arrival process. 
(c) Thinning of an arrival process: Under this operation, a fraction $f$ of arrivals from a given arrival process is classified as type I while the remaining arrivals are classified as type II. In Theorem 9, we characterize the uncertainty set of the resulting thinned type I process.

\subsubsection{The Superposition Process}

Let us consider a queue $j$ that is fed by $m$ arrival processes. Let $\mathcal{U}_{j}^{a}$ denote the uncertainty set representing the inter-arrival times $\left\{T_{i}^{j}\right\}_{i \geq 1}$ from arrival process $j=$ $1, \ldots, m$. We denote the uncertainty set of the combined arrival process by $\mathcal{U}_{s u p}^{a}$. Given the primitives $\left(\lambda_{j}, \Gamma_{a, j}, \alpha\right), j=1, \ldots, m$, we define the superposition operator

$$
\left(\lambda_{\text {sup }}, \Gamma_{a, s u p}, \alpha_{\text {sup }}\right)=\text { Combine }\left\{\left(\lambda_{j}, \Gamma_{a, j}, \alpha\right), j=1, \ldots, m\right\}
$$

where $\left(\lambda_{s u p}, \Gamma_{a, s u p}, \alpha_{s u p}\right)$ characterize the merged arrival process $\left\{T_{i}\right\}_{i \geq 1}$.

Theorem 8 (Superposition Operator). The superposition of arrival processes characterized by the uncertainty sets

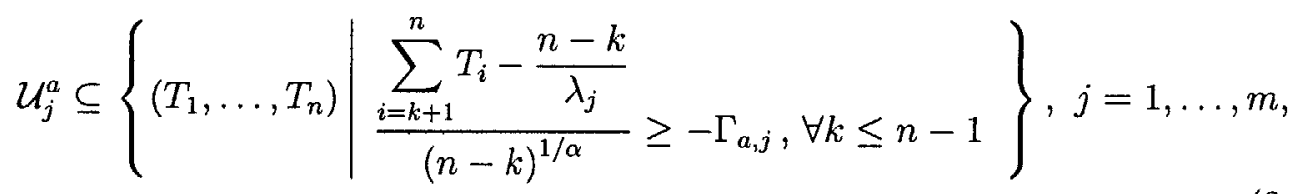

results in a merged arrival process characterized by the uncertainty set

$$
\mathcal{U}_{\text {sup }}^{a}=\left\{\begin{array}{l|l}
\left(T_{1}, \ldots, T_{n}\right) \mid \frac{\sum_{i=k+1}^{n} T_{i}-\frac{n-k}{\lambda_{s u p}}}{(n-k)^{1 / \alpha}} \geq-\Gamma_{a, s u p}, \forall k \leq n-1
\end{array}\right\}
$$

where

$$
\lambda_{s u p}=\sum_{j=1}^{m} \lambda_{j}, \quad \alpha_{s u p}=\alpha, \Gamma_{a, s u p}=\frac{\left(\sum_{j=1}^{m}\left(\lambda_{j} \Gamma_{a, j}\right)^{\alpha /(\alpha-1)}\right)^{(\alpha-1) / \alpha}}{\sum_{j=1}^{m} \lambda_{j}} .
$$


Proof. We first provide a proof for the case where $m=2$, and then generalize the result through induction.

(a) Let $\left\{T_{i}^{j}\right\}_{i \geq 1} \in \mathcal{U}_{j}^{a}, j=1,2$ where $\mathcal{U}_{j}^{a}$ is given by Eq. (2.42), that is

$$
\lambda_{j} \sum_{i=k_{j}+1}^{n_{j}} T_{i}^{j} \geq\left(n_{j}-k_{j}\right)-\lambda_{j} \Gamma_{a, j}\left(n_{j}-k_{j}\right)^{1 / \alpha}, j=1,2
$$

Summing over index $j=1,2$, we obtain

$$
\begin{gathered}
\lambda_{1} \sum_{i=k_{1}+1}^{n_{1}} T_{i}^{1}+\lambda_{2} \sum_{i=k_{2}+1}^{n_{2}} T_{i}^{2} \geq\left(n_{1}-k_{1}+n_{2}-k_{2}\right)-\lambda_{1} \Gamma_{a, 1}\left(n_{1}-k_{1}\right)^{1 / \alpha} \\
-\lambda_{2} \Gamma_{a, 2}\left(n_{2}-k_{2}\right)^{1 / \alpha}
\end{gathered}
$$

Without loss of generality, consider the time window $T$ between the arrival of the $k_{1}^{\text {th }}$ and the $n_{1}^{\text {th }}$ jobs from arrival process 1 , and assume that, within period $T$, the queue sees arrivals of jobs $\left(k_{2}+1\right)$ up to $\left(n_{2}-1\right)$ from arrival process 2 ,

$$
T=\sum_{i=k_{1}+1}^{n_{1}} T_{i}^{1} \leq \sum_{i=k_{2}+1}^{n_{2}} T_{i}^{2}
$$

During time window $T$, the queue receives a total of $\left(n_{1}-k_{1}+n_{2}-k_{2}\right)$ jobs, with $\left(n_{1}-k_{1}+1\right)$ arrivals detected from the first arrival process (including job $\left.k_{1}\right)$, and $\left(n_{2}-k_{2}-1\right)$ arrivals from second arrival process. Therefore, period $T$ can also be written in terms of the combined inter-arrival times $\left\{T_{i}\right\}_{i \geq 1}$ as

$$
T=\sum_{i=k+1}^{n} T_{i}, \text { where } k=k_{1}+k_{2}, \text { and } n=n_{1}+n_{2} .
$$

Combining Eqs. (2.45) and (2.46) yields

$$
\left(\lambda_{1}+\lambda_{2}\right) \sum_{i=k+1}^{n} T_{i} \geq \lambda_{1} \sum_{i=k_{1}+1}^{n_{1}} T_{i}^{1}+\lambda_{2} \sum_{i=k_{2}+1}^{n_{2}} T_{i}^{2}
$$


which by Eq. (2.44) can be written as

$$
\left(\lambda_{1}+\lambda_{2}\right) \sum_{i=k+1}^{n} T_{i} \geq(n-k)-\lambda_{1} \Gamma_{a, 1}\left(n_{1}-k_{1}\right)^{1 / \alpha}-\lambda_{2} \Gamma_{a, 2}\left(n_{2}-k_{2}\right)^{1 / \alpha}
$$

Rearranging and dividing both sides by $\left(\lambda_{1}+\lambda_{2}\right)$ and $(n-k)^{1 / \alpha}$, we ontain

$$
\begin{gathered}
\frac{\sum_{i=k+1}^{n} T_{i}-\frac{n-k}{\lambda_{\text {sup }}}}{(n-k)^{1 / \alpha}} \geq-\Gamma_{a, \text { sup }}(n, k), \text { where } \lambda_{\text {sup }}=\lambda_{1}+\lambda_{2}, \alpha_{\text {sup }}=\alpha \text {, and } \\
\Gamma_{a, \text { sup }}(n, k)= \\
+\frac{\lambda_{1}}{\lambda_{1}+\lambda_{2}} \Gamma_{a, 1}\left(\frac{n_{1}-k_{1}}{n_{1}-k_{1}+n_{2}-k_{2}}\right)^{1 / \alpha} \\
+\frac{\lambda_{2}}{\lambda_{1}+\lambda_{2}} \Gamma_{a, 2}\left(\frac{n_{2}-k_{2}}{n_{1}-k_{1}+n_{2}-k_{2}}\right)^{1 / \alpha} .
\end{gathered}
$$

By letting

$$
x=\frac{n_{1}-k_{1}}{n_{1}-k_{1}+n_{2}-k_{2}}
$$

the maximum value that $\Gamma_{a, s u p}(n, k)$ can achieve over the range of $(n, k)$ can be determined by optimizing the following one-dimensional concave maximization problem over $x \in(0,1)$

$$
\max _{x \in(0,1)}\left\{\beta x^{1 / \alpha}+\delta(1-x)^{1 / \alpha}\right\}=\left(\beta^{\alpha /(\alpha-1)}+\delta^{\alpha /(\alpha-1)}\right)^{(\alpha-1) / \alpha}
$$

where

$$
\beta=\frac{\lambda_{1}}{\lambda_{1}+\lambda_{2}} \Gamma_{a, 1}, \text { and } \delta=\frac{\lambda_{2}}{\lambda_{1}+\lambda_{2}} \Gamma_{a, 2}
$$

Substituting $\beta$ and $\delta$ by their respective values in Eq. (2.49) completes the proof for $m=2$ with

$$
\Gamma_{a, \text { sup }}=\frac{\left[\left(\lambda_{1} \Gamma_{a, 1}\right)^{\alpha /(\alpha-1)}+\left(\lambda_{2} \Gamma_{a, 2}\right)^{\alpha /(\alpha-1)}\right]^{(\alpha-1) / \alpha}}{\lambda_{1}+\lambda_{2}} .
$$

We refer to this procedure of combining two arrival processes by the operator

$$
\left(\lambda_{\text {sup }}, \Gamma_{a, \text { sup }}, \alpha_{\text {sup }}\right)=\text { Combine }\left\{\left(\lambda_{1}, \Gamma_{a, 1}, \alpha\right),\left(\lambda_{2}, \Gamma_{a, 2}, \alpha\right)\right\}
$$


(b) Suppose that the arrivals to a queue come from arrival processes 1 through $(m-1)$. We assume that the combined arrival process belongs to the proposed uncertainty set, with

$$
\bar{\lambda}=\sum_{j=1}^{m-1} \lambda_{j} \text { and } \bar{\Gamma}_{a}=\frac{\left(\sum_{j=1}^{m-1}\left(\lambda_{j} \Gamma_{a, j}\right)^{\alpha /(\alpha-1)}\right)^{(\alpha-1) / \alpha}}{\bar{\lambda}} .
$$

Extending the proof to $m$ sources can be easily done by repeating the procedure shown in part (a) through the operator

$$
\left(\lambda_{\text {sup }}, \Gamma_{a, s u p}, \alpha_{\text {sup }}\right)=\text { Combine }\left\{\left(\bar{\lambda}, \bar{\Gamma}_{a}, \alpha\right),\left(\lambda_{m}, \Gamma_{a, m}, \alpha\right)\right\}
$$

This concludes the proof.

\subsubsection{The Thinning Process}

We consider an arrival process $\left\{T_{i}\right\}_{i \geq 1}$ in which a fraction $f$ of arrivals are classified as type I and the remaining are classified as type II. Moreover, since we consider deterministic splitting, the values of $f$ are necessarily rational by implementation.Given the primitives $\left(\lambda, \Gamma_{a}\right)$ of the original process and the fraction $f$, we define the thinning operator

$$
\left(\lambda_{s p l i t}, \Gamma_{a, s p l i t}, \alpha\right)=\operatorname{Split}\left\{\left(\lambda, \Gamma_{a}, \alpha\right), f\right\}
$$

where $\left(\lambda_{\text {split }}, \Gamma_{a, s p l i t}, \alpha\right)$ characterizes the thinned arrival process $\left\{T_{i}^{s p l i t}\right\}_{i \geq 1}$.

Theorem 9 (Thinning Operator). The thinned arrival process of a fraction $f$ of arrivals belonging to $\mathcal{U}^{a}$ is described by the uncertainty set

$$
\mathcal{U}_{s p l i t}^{a} \subseteq\left\{\begin{array}{l|l}
\left\{T_{i}^{s p l i t}\right\}_{i \geq 1} & \frac{\sum_{i=k+1}^{n} T_{i}^{s p l i t}-\frac{n-k}{\lambda_{\text {split }}}}{(n-k)^{1 / \alpha}} \geq-\Gamma_{a, s p l i t}, \quad k \leq n-1
\end{array}\right\}
$$

where $\lambda_{\text {split }}=\lambda \cdot f$ and $\Gamma_{a, s p l i t}=\Gamma_{a} \cdot\left(\frac{1}{f}\right)^{1 / \alpha}$.

Proof. Consider an arrival process described by $\mathcal{U}^{a}$ and consider the time window 
between the $k^{t h}$ and the $n^{\text {th }}$ arrivals. Suppose that a fraction $f$ of these arrivals are type I arrivals, i.e. , out of the total of $(n-k)$ arrivals excluding the $k^{\text {th }}$ job, $\left(n_{\text {split }}-k_{\text {split }}\right)$ are type I arrivals, such that

$$
f=\frac{n_{s p l i t}-k_{\text {split }}}{n-k}
$$

Let $\left\{T_{i}^{s p l i t}\right\}_{i \geq 1}$ denote the inter-arrival times in the thinned arrival process. Note that

$$
\sum_{i=k_{s p l i t}+1}^{n_{s p l i t}} T_{i}^{s p l i t}=\sum_{i=k+1}^{n} T_{i}
$$

with equality satisfied when the $k^{\text {th }}$ and $n^{\text {th }}$ jobs are both classified as type I. By Assumption 1, we obtain

$$
\sum_{i=k_{s p l i t}+1}^{n_{\text {split }}} T_{i}^{s p l i t} \geq \frac{n-k}{\lambda}-(n-k)^{1 / \alpha} \Gamma_{a}
$$

We obtain the upper bound in Eq. (2.50) by substituting $(n-k)$ by $\left(n_{\text {split }}-k_{\text {split }}\right) / f$. This concludes the proof.

Remark: The superposition and thinning operators are consistent. In fact, it is easy to check that, for splitting fractions $f_{j}$ such that $\sum_{j=1}^{m} f_{j}=1$,

$$
\text { Combine }\left\{\operatorname{Split}\left\{\left(\lambda, \Gamma_{a}, \alpha\right), f_{j}\right\}, j=1, \ldots, m\right\}=\left(\lambda, \Gamma_{a}, \alpha\right)
$$

\subsubsection{The Overall Network Characterization}

Having built an understanding of the superposition, departure and thinning processes, we are now in a position to present our approach for performing exact analysis of queueing networks, which is a major contribution of this paper. We perceive the queueing network as a collection of independent queues that could be analyzed separately. We employ the Combine and Split operators in view of characterizing the effective arrival process to each queue in the network. Knowledge of the effective 
arrival process allows to study the jobs' waiting time at this queue through Theorems 4 and 5 as well as its departure process through Theorems 6 and 7 for a single-server and multi-sever queue, respectively. Theorem 10 characterizes the effective arrival process perceived at each queue in the network.

Theorem 10. The behavior of a single class queueing network is equivalent to that of a collection of independent queues, with the arrival process to node $j$ characterized by the uncertainty set

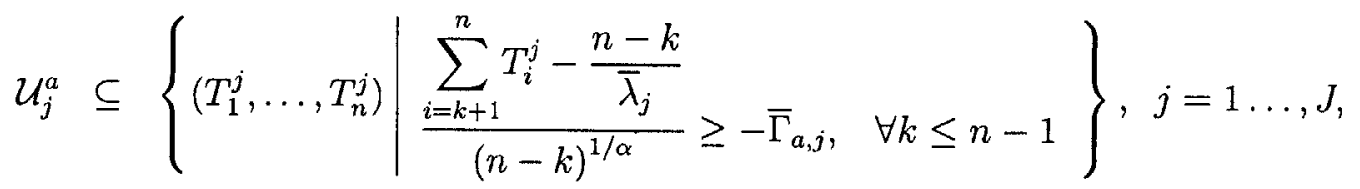

where $\left\{\bar{\lambda}_{1}, \bar{\lambda}_{2}, \ldots, \bar{\lambda}_{J}\right\}$ and $\left\{\bar{\Gamma}_{a, 1}, \bar{\Gamma}_{a, 2}, \ldots, \bar{\Gamma}_{a, j}\right\}$ satisfy the set of equations for all $j=1, \ldots, J$

$$
\begin{aligned}
\bar{\lambda}_{j} & =\lambda_{j}+\sum_{i=1}^{J}\left(\bar{\lambda}_{i} f_{i j}\right) \\
\bar{\Gamma}_{a, j} & =\frac{\left[\left(\lambda_{j} \cdot \Gamma_{a, j}\right)^{\alpha /(\alpha-1)}+\sum_{i=1}^{J}\left(\bar{\lambda}_{i} \cdot \bar{\Gamma}_{a, i}\right)^{\alpha /(\alpha-1)} \cdot f_{i j}\right]^{(\alpha-1) / \alpha}}{\bar{\lambda}_{j}}
\end{aligned}
$$

Proof. Let us consider a queue $j$ receiving jobs from

(a) external arrivals described by parameters $\left(\lambda_{j}, \Gamma_{a, j}, \alpha\right)$, and

(b) internal arrivals routed from queues $i, i=1, \ldots, J$ resulting from splitting the effective departure process from queue $i$ by $f_{i j}$. By Theorems 6 and 7 , the effective departure process from queue $i$ has the same form as the effective arrival process to queue $i$ described by the parameters $\left(\bar{\lambda}_{i}, \bar{\Gamma}_{a, i}, \alpha\right)$.

The effective arrival process to queue $j$ can therefore be represented as

$$
\left(\bar{\lambda}_{j}, \bar{\Gamma}_{a, j}, \alpha\right)=\text { Combine }\left\{\left(\lambda_{j}, \Gamma_{a, j}, \alpha\right),\left(\operatorname{Split}\left\{\left(\bar{\lambda}_{i}, \bar{\Gamma}_{a, i}, \alpha\right), f_{i j}\right\}\right), i=1, \ldots, J\right\}
$$


By Theorem 9, we substitute the split processes by their resulting parameters and obtain the superposition of $J+1$ arrival processes

$$
\left(\bar{\lambda}_{j}, \bar{\Gamma}_{a, j}, \alpha\right)=\text { Combine }\left\{\left(\lambda_{j}, \Gamma_{a, j}, \alpha\right),\left(f_{i j} \bar{\lambda}_{i}, \bar{\Gamma}_{a, i}\left(\frac{1}{f_{i j}}\right)^{1 / \alpha}, \alpha\right), i=1, \ldots, J\right\}
$$

Applying now Theorem 8 yields Eqs. (2.51) and (2.52).

Note that in our analysis, we have assumed that each queue in the network perceives one stream of external arrivals. However, Theorem 10 can be extended in the case where external arrivals are thinned among different queues in the network. This can be done by adding a node in the network for each thinned external arrival process and appending its thinning probabilities to the transition matrix $\mathbf{F}$. We now provide the main insights and implications that arise from Theorem 10.

(a) Network Performance Analysis: Theorem 10 allows us to compute performance measures in a queueing network by considering the queues separately. For instance, the waiting time $\widehat{W}_{n}^{j}$ at queue $j$ can be determined through Theorems 4 and 5 with an effective arrival parameters $\left(\bar{\lambda}_{j}, \bar{\Gamma}_{a, j}, \alpha\right)$ and service parameters $\left(\mu, \Gamma_{s}, \alpha\right)$.

(b) Tractable System Solution: Determining the overall network parameters $(\bar{\lambda}, \bar{\Gamma})$ amounts to solving a set of linear equations. To see this, substitute $x_{j}=$ $\left(\bar{\lambda}_{j} \bar{\Gamma}_{a, j}\right)^{\alpha /(\alpha-1)}$, for all $j=1, \ldots, J$, in Eqs. (2.51) and (2.52) to obtain the following linear system of equations

$$
\begin{cases}\bar{\lambda}_{j}=\lambda_{j}+\sum_{i=1}^{J} \bar{\lambda}_{i} f_{i j} & j=1, \ldots, J \\ x_{j}=\left(\lambda_{j} \Gamma_{a, j}\right)^{\alpha /(\alpha-1)}+\sum_{i=1}^{J} f_{i j} x_{i} & j=1, \ldots, J .\end{cases}
$$

Given that the routing matrix $\mathbf{F}=\left\{f_{i j}\right\}$ is sub-stochastic, the linear system of equations solves for $\left(\bar{\lambda}_{j}, x_{j}\right)$, hence allowing to determine $\bar{\Gamma}_{a, j}$, for all $j=1, \ldots, J$. 


\subsection{Queues with Asymmetric Heavy-tailed Arrival and Service Processes}

In this section, we extend our results in Sections 3-5 for the case of asymmetric heavytailed arrival and service processes, that is, when $\alpha_{a} \neq \alpha_{s}$. We present analogs of Theorems 4-10 that allow us to analyze queueing networks composed of queues with arbitrary values for $\alpha_{a}$ 's and $\alpha_{s}$ 's. In this section, we let $\mathbf{1}_{\{x=y\}}$ denote the indicator variable defined by

$$
1_{\{x=y\}}= \begin{cases}1, & \text { if } x=y \\ 0, & \text { otherwise. }\end{cases}
$$

We omit the proofs as they are straightforward generalizations of the proofs in Theorems $4-10$.

\section{Waiting Times with $\alpha_{a} \neq \alpha_{s}$}

As discussed in Section 3, we compute the waiting times $\widehat{W}$ by solving the optimization problem

$$
\max _{x} \beta x^{1 / \alpha_{a}}+\delta x^{1 / \alpha_{s}}-\gamma x
$$

where $\beta=m^{1 / \alpha_{a}} \Gamma_{a}, \delta=\Gamma_{s}$, and $\gamma=m(1-\rho) / \lambda$. Problem (2.55) is a concave maximization problem, which can be solved numerically using Newton's method. We next present a bound on the waiting time, which is asymptotically tight as $\rho \rightarrow 1$, but not necessarily tight for other $\rho$.

Theorem 11. If $\left\{T_{i}\right\}_{i \geq 1} \in \mathcal{U}^{a},\left\{X_{i}\right\}_{i \geq 1} \in \mathcal{U}_{m}^{s}$ and $\rho<1$, then

$$
\widehat{W} \leq \frac{\bar{\alpha}-1}{\bar{\alpha}^{\bar{\alpha} /(\bar{\alpha}-1)}} \cdot \frac{\lambda^{1 /(\bar{\alpha}-1)}\left(\Gamma_{a}+\Gamma_{s} / m^{1 / \bar{\alpha}}\right)^{\bar{\alpha} /(\bar{\alpha}-1)}}{(1-\rho)^{1 /(\bar{\alpha}-1)}}
$$

where $\bar{\alpha}=\min \left(\alpha_{a}, \alpha_{s}\right)$.

Note that the exponent of $(1-\rho)$ depends on the $\bar{\alpha}$ term corresponding to the heaviest among the arrival and service processes. 


\section{Superposition Process}

In Section 5, we have assumed that all the combining arrival processes have the same value for $\alpha_{a}=\alpha$. We next consider the case when the arrival streams are characterized by different $\alpha^{\prime} s$.

Theorem 12. The superposition of arrival processes characterized by the uncertainty sets

$$
\mathcal{U}_{j}^{a}=\left\{\begin{array}{l|l}
\left(T_{1}, \ldots, T_{n}\right) & \frac{\sum_{i=k+1}^{n} T_{i}-\frac{n-k}{\lambda_{j}}}{(n-k)^{1 / \alpha_{a, j}}} \geq-\Gamma_{a, j}, \quad \forall k \leq n-1
\end{array}\right\}, j=1, \ldots(2 \pi n 6)
$$

results in a merged arrival process characterized by the uncertainty set

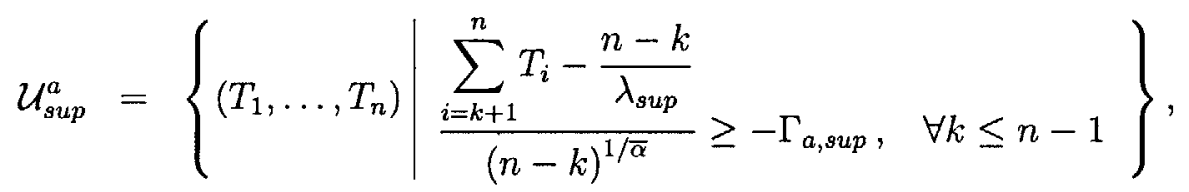

where

$$
\lambda_{\text {sup }}=\sum_{j=1}^{m} \lambda_{j}, \bar{\alpha}=\min _{j} \alpha_{a, j}, \Gamma_{a, \text { sup }}=\frac{\left(\sum_{j=1}^{m} 1_{\left\{\alpha_{a, j}=\bar{\alpha}\right\}} \cdot\left(\lambda_{j} \Gamma_{a, j}\right)^{\alpha_{a, j} /\left(\alpha_{a, j}-1\right)}\right)^{(\bar{\alpha}-1) / \bar{\alpha}}}{\sum_{j=1}^{m} \lambda_{j}} .
$$

\section{Thinning Process}

We note that the split arrival process inherits the $\alpha_{a}$ term corresponding to the thinned arrival process. Hence, Theorem 9 still holds in this case.

\section{The Generalized Queueing Network}

We consider a queueing network and characterize the parameters of the effective arrival processes to each queueing node in the network under the assumption of asymmetric tail behavior. We observe that the parameter $\bar{\alpha}_{a, j}$ describing the tail behavior 
of the effective arrival process depends on the tail behavior of all the queueing nodes that communicate with node $j$.

Theorem 13. Consider a queueing network with $J$ queues and external arrival processes characterized by $\left(\lambda_{j}, \Gamma_{a, j}, \alpha_{a, j}\right)$. The behavior of this network is equivalent to that of a collection of independent queues, with the arrival process to node $j$ characterized by the uncertainty set

$$
\overline{\mathcal{U}}_{j}^{a} \subseteq\left\{\begin{array}{l|l}
\left(T_{1}, \ldots, T_{n}\right) & \frac{\sum_{i=k+1}^{n} T_{i}-\frac{n-k}{\bar{\lambda}_{j}}}{(n-k)^{1 / \bar{\alpha}_{a, j}}} \geq-\bar{\Gamma}_{a, j}, \quad \forall k \leq n-1
\end{array}\right\}, j=1 \ldots, J
$$

where $\left\{\bar{\lambda}_{1}, \bar{\lambda}_{2}, \ldots, \bar{\lambda}_{J}\right\}$ and $\left\{\bar{\Gamma}_{a, 1}, \bar{\Gamma}_{a, 2}, \ldots, \bar{\Gamma}_{a, J}\right\}$ satisfy the set of equations for all $j=1, \ldots, J$

$$
\begin{aligned}
\bar{\lambda}_{j} & =\lambda_{j}+\sum_{i=1}^{J}\left(\bar{\lambda}_{i} f_{i j}\right) \\
\bar{\Gamma}_{a, j} & =\frac{\left[\left(\lambda_{j} \cdot \Gamma_{a, j}\right)^{\alpha_{a, j} /\left(\alpha_{a, j}-1\right)}+\sum_{i=1}^{J}\left(\bar{\lambda}_{i} \cdot \bar{\Gamma}_{a, i}\right)^{\bar{\alpha}_{a, i} /\left(\bar{\alpha}_{a, i}-1\right)} \cdot f_{i j}\right]^{\left(\bar{\alpha}_{a, j}-1\right) / \bar{\alpha}_{a, j}}}{\bar{\lambda}_{j}}
\end{aligned}
$$

with $\bar{\alpha}_{a, j}=\min _{i:\{i \rightarrow j\}} \alpha_{a, i}$, where $\{i \rightarrow j\}$ means that node $i$ communicates with node $j$ in the network with routing matrix $\mathbf{F}$.

We provide the main insights and implications that arise from Theorem 13.

(a) Effect of Heavier Tails: Theorem 13 implies that the tail behavior of the effective arrival process at a given queue is determined by the "heaviest" tail among all departure processes arriving to this queue including the external arrival process to the queue. If all nodes communicate with each other, the tail behavior of the queueing network is then determined by the heaviest tail among the external arrival processes.

(b) Tractable System Solution: Note that the set of equations that characterize the effective arrival process are similar to Eqs. (2.51) and (2.52). The only 
difference in the system for the asymmetric case is the presence of indicator variables $1_{\left\{\alpha_{a, j}=\bar{\alpha}_{a, j}\right\}}$ which isolate the heaviest tail among the merged arrival processes at any given node. Given that these indicator variables are known from data, one could think of this system as a linear system of equations (as for Eqs. (2.51) and (2.52)) with $\tilde{f}_{i j}=\mathbf{1}_{\left\{\bar{\alpha}_{a, i}=\bar{\alpha}_{a, j}\right\}} \cdot f_{i j}$. The modified routing matrix with entries $\tilde{f}_{i j}$ remains sub-stochastic allowing a unique solution to this system of linear equations.

\subsection{Computational Results}

In this section, we present computational results to demonstrate the effectiveness of our approach in analyzing queueing networks. We shall refer to our approach as the Robust Queueing Network Analyzer (RQNA) in the remainder of this chapter. Our objectives consist of

(1) comparing the results obtained by RQNA with the results obtained from simulation and the Queueing Network Analyzer (QNA) proposed by Whitt [1983], and

(2) investigating the relative performance of RQNA with respect to system's network size, degree of feedback, maximum traffic intensity, and diversity of external arrival distributions.

In view of comparing our approach to simulation and QNA, we consider instances of stochastic queueing networks with the following primitive data:

(a) The distributions of the external arrival processes with parameters $\left(\lambda_{j}, \sigma_{a, j}, \alpha_{a, j}\right)$ with coefficients of variation $c_{a, j}^{2}=\lambda_{j}^{2} \sigma_{a, j}^{2}, j=1, \ldots, J$.

(b) The distributions of the service processes with parameters $\left(\mu_{j}, \sigma_{s, j}, \alpha_{s, j}\right)$ with coefficients of variation $c_{s, j}^{2}=\mu_{j}^{2} \sigma_{s, j}^{2}$ and the number of servers $m_{j}, j=1, \ldots, J$.

(c) Routing matrix $\mathbf{F}=\left[f_{i j}\right], i, j=1, \ldots, J$, where $f_{i j}$ denotes the fraction of jobs passing through queue $i$ routed queue $j$. The fraction of jobs leaving the network 
from queue $i$ is $1-\sum_{j} f_{i j}$

To apply RQNA on stochastic queueing networks, we first need to translate the stochastic primitive data given above into robust primitive data, namely uncertainty sets with appropriate variability parameters $\left(\Gamma_{a, j}, \Gamma_{s, j}\right)$ for each $j=1, \ldots, J$. To achieve this goal, we first describe in subsection 2.6.1 how we use simulation on a single isolated queue to construct parameters $\left(\Gamma_{a}, \Gamma_{s}\right)$ given arrival and service distributions. This enables us to transform the stochastic data into uncertainty sets over external arrival and service processes. We then present in subsection 2.6.2 an algorithm that details the procedure employed by RQNA to compute the desired performance measures for a network of queues. In subsection 2.6.3, we report on the performance of RQNA in comparison to QNA and simulation, while in subsection 2.6.4, we discuss on the performance of RQNA as a function of various network parameters.

\subsubsection{Derived Variability Parameters}

Along the lines of QNA, we use simulation to construct appropriate functions for the variability parameters. To do so, we consider a single queue with $m$ servers characterized by $\left(\rho, \sigma_{a}, \sigma_{s}, \alpha_{a}, \alpha_{s}\right)$ and model its variability parameters $\left(\Gamma_{a}, \Gamma_{s}\right)$ as follows

$$
\Gamma_{a}=\sigma_{a} \text { and } \Gamma_{s}=f\left(\rho, \sigma_{a}, \sigma_{s}, \alpha_{a}, \alpha_{s}\right)
$$

Motivated by Kingman's bound (see Eq. (2.4)), we consider the following functional form for $f(\cdot)$

$$
f\left(\rho, \sigma_{s}, \sigma_{a}, \alpha_{a}, \alpha_{s}\right)=\left(\theta_{0}+\theta_{1} \cdot \sigma_{s}^{2} / m+\theta_{2} \cdot \sigma_{a}^{2} \rho^{2} m\right)^{(\alpha-1) / \alpha}-\sigma_{a} m^{(\alpha-1) / \alpha},
$$

where $\alpha=\min \left\{\alpha_{a}, \alpha_{s}\right\}$. We run simulation over multiple instances of a single queue while varying parameters $\left(\rho, \sigma_{a}, \sigma_{s}, \alpha_{a}, \alpha_{s}\right)$ for different arrival and service distributions. We employ linear regression to generate appropriate values for $\theta_{0}, \theta_{1}$ and $\theta_{2}$

such that the values obtained for $\widehat{W}_{n}$ by Theorem 11 are adapted according to the ex- 
pected values of the waiting time obtained from simulation. We propose two different adaptation regimes:

(a) Service Distribution Dependent Adaptation Regime where we allow the set of values

$$
\left(\theta_{0}, \theta_{1}, \theta_{2}\right)
$$

to depend on the service distribution, and

(b) Service Distribution Independent Adaptation Regime where we obtain a single set of values $\left(\theta_{0}, \theta_{1}, \theta_{2}\right)$.

We would naturally expect that knowledge of the specific service distributions leads to more accurate answers and indeed this is verified by the results below. The motivation for considering the service independent adaptation regime is that often we might not know the service time distributions. We also note that we do not perform an adaptation of the values of $\left(\theta_{0}, \theta_{1}, \theta_{2}\right)$ for each arrival distribution, since in the network, we have no prior knowledge of the arrival distribution at a given queue. The only known distribution at each queue is in fact the service distribution, hence the proposed adaptation methods. We report the values obtained for both adaptation regimes in Table 2.1. Using the tabulated values for $\left(\theta_{0}, \theta_{1}, \theta_{2}\right)$, Tables 2.2 and 2.3

\begin{tabular}{cccc}
\hline$\left(\theta_{0}, \theta_{1}, \theta_{2}\right)$ & \multicolumn{2}{c}{ Service Dependent } & Service Independent \\
& Pareto & Normal & \\
\hline$\theta_{0}$ & -0.05 & -0.02 & -0.063 \\
$\theta_{1}$ & 1.09 & 1.0301 & 1.072 \\
$\theta_{2}$ & 1.11 & 1.0409 & 1.068 \\
\hline
\end{tabular}

Table 2.1: Adapted $\theta$ values.

present the percentage errors of the waiting times obtained by Theorem 11 relative to the expected waiting time values obtained by simulation for a single queue with multiple servers.

We observe that errors are within $8 \%$ and $9.5 \%$ of simulation for the single-server and multi-server queue, respectively. Note that adapting for the different service distributions leads to smaller errors in comparison to the service independent adaptation 


\begin{tabular}{ccccc}
\hline Case & \multicolumn{2}{c}{ Pareto Distribution } & \multicolumn{2}{c}{ Normal Distribution } \\
$\left(c_{a}^{2}, c_{s}^{2}\right)$ & RQNA $^{*}$ & RQNA** & RQNA $^{*}$ & RQNA $^{* *}$ \\
\hline$(0.25,0)$ & 6.812 & 2.912 & 6.547 & 1.185 \\
$(0.25,1)$ & -7.314 & -3.080 & 6.483 & 3.246 \\
$(0.25,4)$ & -6.583 & -2.708 & -7.268 & -3.172 \\
$(1,0)$ & 7.665 & 0.982 & -5.505 & -2.960 \\
$(1,1)$ & 6.423 & 1.357 & -6.579 & -2.114 \\
$(1,4)$ & 6.123 & 2.485 & 6.070 & -1.532 \\
$(4,0)$ & -5.701 & -1.729 & 6.050 & 1.380 \\
$(4,1)$ & -7.839 & -1.824 & -7.434 & -3.084 \\
$(4,4)$ & -5.886 & -2.231 & 7.405 & 1.334 \\
\hline
\end{tabular}

Table 2.2: Percentage errors for single server queues using RQNA. (* Service Independent $\quad{ }^{* *}$ Service Dependent)

\begin{tabular}{ccccc}
\hline Case & \multicolumn{2}{c}{3 servers } & \multicolumn{2}{c}{ 10 servers } \\
$\left(c_{a}^{2}, c_{s}^{2}\right)$ & Normal & Pareto & Normal & Pareto \\
\hline$(0.25,0)$ & 8.822 & -9.356 & 8.453 & -9.269 \\
$(0.25,1)$ & 7.238 & -7.957 & 9.264 & -8.839 \\
$(0.25,4)$ & -8.195 & 9.856 & -8.842 & 8.894 \\
$(1,0)$ & -10.360 & -8.833 & -8.508 & -9.420 \\
$(1,1)$ & -7.985 & 8.929 & -8.583 & 9.365 \\
$(1,4)$ & 8.548 & 8.255 & 7.978 & 9.046 \\
$(4,0)$ & -7.296 & 7.865 & -9.209 & 9.514 \\
$(4,1)$ & -7.593 & -7.913 & -8.739 & -9.553 \\
$(4,4)$ & -9.372 & -8.776 & -8.960 & -9.338 \\
\hline
\end{tabular}

Table 2.3: Percentage errors for multi-server queues using RQNA.

regime, with errors within $3 \%$ and $7.5 \%$ for the single-server and multi-server queue, respectively.

\subsubsection{The RQNA Algorithm}

Having derived the required primitive data for our robust approach, we next describe the RQNA algorithm we employ to compute performance measures of a given network of queues.

\section{ALGORITHM 1. Robust Queueing Network Analyzer}


Input:] External arrival parameters $\left(\lambda_{j}, \sigma_{a, j}, \alpha_{a, j}\right)$, service parameters $\left(\mu_{j}, \sigma_{s, j}, \alpha_{s, j}\right)$, and routing matrix $\mathbf{F}=\left[f_{i j}\right]$, for $i, j=1, \ldots, J$. Input also the service times distributions for the case of service dependent adaptation regime.

Output: Waiting times $\widehat{W}$ at each node $j, j=1, \ldots, J$.

1. For each external arrival process $i$ in the network, set $\Gamma_{a, i}=\sigma_{a, i}$.

2. For each queue $j$ in the network with parameters $\left(\mu_{j}, \sigma_{s, j}, \alpha_{s, j}\right)$, compute

- (a) the effective parameters $\left(\bar{\lambda}_{j}, \bar{\Gamma}_{a, j}, \bar{\alpha}_{a, j}\right)$ according to Theorem 13 and set $\rho_{j}=\bar{\lambda}_{j} / \mu_{j}$

- (b) the variability parameter $\Gamma_{s, j}=f\left(\rho_{j}, \bar{\Gamma}_{a, j}, \sigma_{s, j}, \bar{\alpha}_{a, j}, \alpha_{s, j}\right)$, and

- (c) the waiting time $\widehat{W}$ at node $j$ using Theorem 11 .

3. Compute the total sojourn time of the network by computing

- (a) the set of all possible paths $\mathcal{P}$ in the network,

- (b) the fraction $f_{p}$ of jobs routed through each path $p \in \mathcal{P}$,

- (c) the corresponding total sojourn time $\widehat{S}_{p}$ across each path $p \in \mathcal{P}$ by summing the

- individual waiting times and service times at all nodes associated with this path,

- (d) the total sojourn time in the network $\widehat{S}=\sum_{p \in \mathcal{P}} f_{p} \widehat{S}_{p}$.

Note that, in Step 2(b), we treat each queue $j$ in the network separately as a single isolated queue with an effective arrival process described by the variability parameter $\bar{\Gamma}_{a, j}$. Note that we use $\bar{\Gamma}_{a, j}$ as an input to $f($.$) in place of the standard deviation.$ This is motivated from our use of $\Gamma_{a}=\sigma_{a}$ for the single queue case (see Eq. (2.57)). It is also possible to compute $\Gamma_{s, j}$ using either the service independent or the service dependent adaptation regime based on whether we know the specific service time distribution at each queue. 


\subsubsection{Performance of RQNA in Comparison to QNA and Simulation}

We consider the Kuehn's Network (see Kuehn [1979]) and perform computations assuming queues have either single or multiple servers, with normal or Pareto distributed service times. Table 2.4 reports the percentage errors between the expected sojourn times calculated by simulation and those obtained by each of QNA and RQNA, assuming all nine queues in the network have a single server. Note that the sojourn time is defined as the time elapsed between the arrival of a job to the network until his departure from the network.

We observe that

(a) RQNA produces results that are often significantly closer to simulated values compared to QNA. Improvements generally range one order of magnitude better in favor of RQNA.

(b) RQNA is fairly insensitive to the heavy-tailed nature of the service distributions. In fact, the sojourn time percentage errors for both the Pareto and normally distributed services are within the same order.

(c) Adaptation of $\left(\theta_{0}, \theta_{1}, \theta_{2}\right)$ to service distribution yields smaller errors up to $6 \%$.

(d) RQNA's performance is generally stable with respect to the number of servers at each queue, yielding errors within the same range for instances with 3 to 10 servers per queue.

\subsubsection{Performance of RQNA as a Function of Network Pa- rameters}

We investigate the performance of RQNA (for the service dependent adaptation regime) as a function of the system's parameters (network size, degree of feedback, maximum traffic intensity among all queues and number of distinct distributions for the external arrival processes) in families of randomly generated queueing networks. 


\begin{tabular}{ccccccc}
\hline $\begin{array}{c}\text { Case } \\
\left(c_{a, j}^{2}, c_{s, j}^{2}\right)\end{array}$ & QNA & $\begin{array}{c}\text { Pareto } \\
\text { RQNA }\end{array}$ & RQNA $^{* *}$ & QNA & $\begin{array}{c}\text { Normal } \\
\text { RQNA }^{*}\end{array}$ & RQNA $^{* *}$ \\
\hline$(0.25,0)$ & 22.78 & 8.281 & 3.291 & 15.28 & 7.786 & 1.389 \\
$(0.25,1)$ & 18.48 & -8.82 & -3.478 & 12.08 & 8.329 & 3.869 \\
$(0.25,4)$ & 20.13 & -7.122 & -3.052 & 11.57 & -7.922 & -3.882 \\
$(1,0)$ & 19.01 & 8.176 & 1.056 & 12.68 & -6.367 & -3.797 \\
$(1,1)$ & 14.06 & 6.832 & 1.799 & 5.84 & -7.125 & -2.555 \\
$(1,4)$ & 10.15 & 6.878 & 2.893 & -10.45 & 7.911 & -0.681 \\
$(4,0)$ & 21.82 & -7.244 & -1.934 & 10.95 & 6.739 & 1.290 \\
$(4,1)$ & 23.71 & -8.729 & -2.139 & 14.18 & -9.28 & -3.508 \\
$(4,4)$ & 17.51 & -7.173 & -2.974 & 11.55 & 9.251 & 1.671 \\
\hline
\end{tabular}

Table 2.4: Percentage errors on Kuehn's network using RQNA. ( ${ }^{*}$ Service Independent ** Service Dependent)

Tables 2.5 and 2.6 report the sojourn time percentage errors of RQNA relative to simulation as a function of the size of the network and the degree of feedback for queues with single and multiple servers, respectively. In the case of multi-server queueing networks, we randomly assign 3,6 or 10 servers to each of the queues in the network independently of each other.

\begin{tabular}{cccccc}
\hline \% Feedback / No of nodes & 10 & 15 & 20 & 25 & 30 \\
\hline Feed-forward networks 0\% & 2.86 & 2.94 & 3.03 & 2.92 & 3.21 \\
$20 \%$ & 3.12 & 3.25 & 3.29 & 3.71 & 3.64 \\
$35 \%$ & 3.74 & 3.81 & 4.02 & 4.07 & 4.14 \\
$50 \%$ & 4.42 & 4.63 & 4.84 & 5.23 & 5.65 \\
$70 \%$ & 4.85 & 5.16 & 5.34 & 5.68 & 5.86 \\
\hline
\end{tabular}

Table 2.5: Sojourn time percentage errors of RQNA relative to simulation as a function of the size of the network and the degree of feedback for queues with single server queueing nodes.

Tables 2.7 and 2.8 present the sojourn time percentage errors for RQNA relative to simulation as a function of the maximum traffic intensity among all queues in the network and the number of distinct distributions for the external arrival processes. Table 2.7 presents the results for networks with only single server queues, while Table 2.8 presents the results for networks in which each queue was randomly assigned 3,6 


\begin{tabular}{cccccc}
\hline \% Feedback / No of nodes & 10 & 15 & 20 & 25 & 30 \\
\hline Feed-forward networks 0\% & 3.594 & 3.546 & 3.756 & 3.432 & 3.846 \\
$20 \%$ & 3.696 & 4.014 & 4.02 & 4.392 & 4.452 \\
$35 \%$ & 4.32 & 4.776 & 4.956 & 5.034 & 4.878 \\
$50 \%$ & 4.95 & 4.806 & 5.358 & 5.67 & 6.192 \\
$70 \%$ & 5.016 & 5.556 & 5.934 & 5.958 & 6.03 \\
\hline
\end{tabular}

Table 2.6: Sojourn time percentage errors of RQNA relative to simulation as a function of the size of the network and the degree of feedback for queues with multi-server queueing nodes.

or 10 servers. Specifically, we design four sets of experiments in which we use one type (normal), two types (Pareto and normal), three types (Pareto, normal and Erlang) and four types (Pareto, normal, Erlang and exponential) of arrival distributions. We observe that

(a) Errors are slightly higher for multi-server networks compared to single-server networks.

(b) RQNA's performance is generally stable for higher degrees of feedback with errors below $6.2 \%$.

(c) RQNA is fairly insensitive to network size with a very slight increase in percent errors between 10-node and 30-node networks.

(d) RQNA presents slightly improved results for lower traffic intensity levels. It is nevertheless fairly stable with respect to higher traffic intensity levels.

(e) The percentage errors generally increase with diversity of external arrival distributions, but still are below $8.5 \%$ relative to simulation.

\subsection{Concluding Remarks}

We revisited in this chapter the problem of analyzing the performance measures of a single-class queue with multiple servers. While the analysis of the $G I / G I / m$ queue is still an open problem under traditional queueing theory, we proposed an uncertainty 


\begin{tabular}{cccccc}
\hline \# different distributions & $\rho=0.95$ & $\rho=0.9$ & $\rho=0.8$ & $\rho=0.65$ & $\rho=0.5$ \\
\hline 1 & 3.34 & 3.26 & 3.17 & 3.02 & 2.72 \\
2 & 6.38 & 5.85 & 5.47 & 4.87 & 3.24 \\
3 & 7.43 & 7.09 & 6.04 & 5.88 & 4.53 \\
4 & 7.56 & 6.98 & 6.81 & 6.29 & 5.18 \\
\hline
\end{tabular}

Table 2.7: Sojourn time percentage errors for RQNA relative to simulation as a function of the maximum traffic intensity and the number of distinct distributions for the external arrival processes for single server queueing networks.

\begin{tabular}{cccccc}
\hline \# different distributions & $\rho=0.95$ & $\rho=0.9$ & $\rho=0.8$ & $\rho=0.65$ & $\rho=0.5$ \\
1 & 4.05 & 4.092 & 3.618 & 3.678 & 3.228 \\
2 & 5.082 & 7.104 & 6.42 & 6.108 & 3.714 \\
3 & 5.916 & 6.318 & 6.9 & 7.344 & 5.676 \\
4 & 7.672 & 8.644 & 7.284 & 6.852 & 5.37 \\
\hline
\end{tabular}

Table 2.8: Sojourn time percentage errors for RQNA relative to simulation as a function of the maximum traffic intensity and the number of distinct distributions for the external arrival processes for multi-server queueing networks.

set model which allows to solve it exactly. In particular, we derive a closed form expression for the waiting times and extend the analysis to arbitrary networks of queues through the following key principle: $a$ ) the departure from a queue, $b$ ) the superposition, and c) the thinning of arrival processes have the same uncertainty set representation as the original arrival processes. Our robust model also tackles heavy-tailed arrival and service processes, yielding closed-form solutions that are not available under traditional queueing theory. We proposed RQNA to analyze queueing networks and found that RQNA (with service dependent adaptation regime) yields results with error percentages in single digits (for all experiments we performed) relative to simulation and performs significantly better than QNA. Moreover, the performance of RQNA is to a large extent insensitive to the number of servers per queue, network size, degree of feedback and traffic intensity, and somewhat sensitive to the degree of diversity of external arrival distributions in the network. We feel that the proposed approach allows us to analyze queueing systems in a tractable way and fulfill the need to obtain both qualitative insights as well numerical tractability that has eluded queueing theory to a large extent in its over 100 years history. It also 
opens the door to analyze more involved queueing systems. Indeed we have extended the proposed approach to performance analysis of queueing systems in the transient domain in Bandi et al. [2012]. 


\section{Chapter 3}

\section{Robust Optimal Auctions}

\subsection{Auction Theory}

The problem of optimal auction design is a central problem in Economics which arises when an auctioneer is interested in selling multiple items to multiple buyers with private valuations for the items. The auctioneer is faced with the task of designing the rules of the auction so as to maximize revenue, while also incentivizing the buyers to reveal their true valuations when they participate in the auction. Building on the work of Vickrey [1961], Myerson [1981] considers the optimal auction design problem for the sale of a single item to buyers with unlimited budgets. He considers this problem in a probabilistic setting, that is, he assumes that the buyers' valuations are drawn from independent, but not necessarily identical, probability distributions. These distributions are assumed to be common knowledge, so that all buyers and the auctioneer know the distribution from which each buyer's valuation is drawn. He obtains a characterization of the optimal solution as a second price auction with buyer dependent reservation prices, which for the special case of identical buyers, reduces to that of a second price auction with a single reservation price.

Given the insightful solution of Myerson, there has been extensive work aimed at solving the following more general auction design problems for buyers with:

(P1) Public budget constraints; 
(P2) Private budget constraints;

(P3) Correlated valuations.

There have been two predominant modeling paradigms in the literature: (a) the probabilistic approach, also referred to as the Bayesian approach in the literature; and (b) the adversarial approach, also referred to as the prior-free approach in the literature. We next present a brief literature review of auction theory with respect to Problems (P1)-(P3), organized around these predominant modeling paradigms.

\subsubsection{Relevant Literature}

Auction theory has always been one of the most active areas of Economics and with the rise of the Internet and E-Commerce, its significance has only increased. In the past decade, auction theory has also attracted the attention of researchers in Theoretical Computer Science. In what follows, we organize our review of the relevant literature around the predominant modeling paradigms mentioned earlier. For a more comprehensive review, we refer the readers to Klemperer [1999], Krishna [2002], Vohra [2011] for the Economics and Nisan et al. [2007] for the Computer Science perspective, respectively.

\section{The Probabilistic Approach}

This approach has been widely studied (see Benoit and Krishna [2001], Brusco and Lopomo [2008], Che and Gale [1996, 2000], Laffont and Robert [1996], Manellia and Vincent [2007], Pai and Vohra [2008], Thanassoulis [2004], Wilson [1997]). The key primitive assumptions are:

(a) Buyers' valuations are sampled from a joint probability distribution;

(b) The auctioneer has exact knowledge of this joint distribution;

(c) The auctioneer is risk neutral and seeks to obtain a mechanism in order to maximize the expected revenue. 
In this setting, we divide the literature based on the problem that was solved.

\section{Public Budget Constraints (Problem P1):}

The analysis of budget constrained auctions was first done by Laffont and Robert [1996], where they assume that all buyers have the same common knowledge budget constraint and derive the subsidy-free (i.e., payments are non-negative) optimal auction. Under the same assumption of equal budgets, Maskin [2000] obtained the optimal auction that maximizes social surplus. Malakhov and Vohra [2004] relaxed the assumption of symmetrical budgets and obtained the revenue maximizing auction for the case of two buyers, only one of whom is budget constrained. Chawla et al. [2010] obtained the first approximation algorithm for the general problem where they show that a sequential all-pay mechanism is a 4-approximation to the revenue of the optimal truthful mechanism with a discrete valuation space for each bidder. They also show that a sequential posted price mechanism is an $O(1)$-approximation to the revenue of the optimal truthful mechanism, when the valuation space of each bidder is a product distribution that satisfies the standard hazard rate condition. Dobzinski et al. [2008] show that an adaptive version of the "clinching auction" (Ausubel [2004]) is Pareto-optimal and incentive compatible. Moreover, they show that it is the unique auction with these properties, when there are exactly two bidders. The more general problem, however, remains open in the setting of public budget constraints under probabilistic assumptions.

\section{Private Budget Constraints (Problem P2):}

Dobzinski et al. [2008] show that there is no incentive compatible, individual rational and Pareto-optimal deterministic auction, for any finite number $m>1$ of units of a single indivisible good and any $n>2$ players, when the budgets are private (see also the discussion in the beginning of Section 3.4. In the same setting, Borgs et al. [2005] showed that it is impossible to design a non-trivial truthful auction which allocates all units. Instead they provide the design of an asymptotically revenue-maximizing truthful mechanism which may allocate only some of the units. Furthermore, Pai 
and Vohra [2008] show several interesting qualitative properties of such auctions by discretizing the valuation space and formulating a linear optimization problem, whose dimension is exponential in the number of buyers. Based on these results, there is a need to consider other notions of optimality in order to obtain numerically tractable auction mechanisms.

Correlated valuations (Problem P3):

For the case of correlated buyers which was left open by Myerson [1981], some of the early work was done by Cremer and McLean [1988] who solved it in a weak sense, that is, using auctions that are individually rational only in expectation. However, the computational complexity of designing the optimal ex-post individually rational auction for correlated valuations has been open until recently, when Papadimitriou and Pierrakos [2011] obtained a polynomial time algorithm for the two buyer case and established an inapproximability result for three or more buyers.

\section{The Adversarial Approach}

The objective in the adversarial approach is to identify a single mechanism that always has good performance, e.g., under any distributional assumption. There have been broadly three approaches that have been used so far:

(a) The resource augmentation approach, also known as, the bicriteria approach which was introduced in Bulow and Klemperer [1996], is based on the observation that in some cases increasing competition, e.g., by recruiting more agents, and running the second price auction mechanism increases revenue when compared to running the (optimal) Myerson mechanism in the original setting.

(b) The main idea in the average-case approach is to show that, for a large class of distributions and settings, there is a single mechanism that approximates the revenue of the Bayesian optimal mechanism. For example, when the probability distribution is known, the second price auction mechanism with a particular way (the so-called monopoly reservation price) of calculating the reservation prices is 
approximately optimal by a constant factor (of 2). Dhangwatnotai et al. [2010] relaxes the need to know the probability distribution of the valuation and uses a sampling-based approach to calculate the reservation price. For the case of correlated buyers, Ronen [2001] proposed a mechanism for the correlated case that achieves half of the optimum revenue.

(c) The worst-case approach, where the idea is to define an appropriate performance benchmark and attempt to obtain mechanisms that approximate this benchmark on any worst-case valuation vector. Goldberg et al. [2006], in a negative result, showed that when the adversary knows all of the buyers' valuations exactly, then no incentive compatible auction can obtain more than a vanishingly small fraction of its revenue in the worst case. Under this approach, it is desirable to identify the right kind of performance benchmarks, but this problem is still open.

All the aforementioned results have been for the cases of buyers without budget constraints and, except for the result in Ronen [2001], they all assume independent valuations. Thus, Problems (P1)-(P3) are open under the adversarial approach.

\subsubsection{Motivation and Contributions}

Our approach is motivated by two considerations. First, we observe that incentive compatibility, see Eq.(3.4), and individual rationality, see Eq.(3.6), key properties of an optimal auction, are inherently robustness properties, that is, properties that have to hold for all possible valuations irrespective of their probability measure. Such properties can be naturally modeled using robust optimization.

Second, we perceive certain shortcomings in the classical probabilistic and adversarial approaches that we reviewed in Section 3.1:

(a) In many cases the only information available to the auctioneer is the previous bidding data. The probabilistic approach chooses to fit a specific probability distribution to this data, which is, in our view, a model of reality, not reality 
itself. In other words, modeling with probability distributions is not inherent to the problem, but rather a choice we make in modeling. On the other hand, the adversarial approach chooses not to make use of this historical bidding data.

(b) Even if the joint probability distribution of the valuations is known or under the adversarial approach, we have seen in Section 3.1 that Problems (P1)-(P3) are by and large open with occasional intractability or even impossibility results.

(c) Even in the case of single item auction without budgets solved by Myerson [1981], there is a possibility of loss of revenue if the auctioneer mis-specifies the probability distribution. Moreover, under the adversarial approach, not making use of historical data again may lead to revenue losses.

Given these considerations, we propose to use the uncertainty set based approach to model the uncertain valuations. Moreover, this approach also allows us to naturally model all the desirable properties (incentive compatibility, individual rationality and budget feasibility) of optimal auctions as robust optimization constraints. In this setting, we propose optimal mechanisms for Problems (P1)-(P3) that involve reservation prices, thus extending the work of Myerson from single item without budgets, to multiple items with budgets, potentially correlated valuations and uncertain budgets. Unlike the Myerson auction where the reservation prices do not depend on the item, the reservation prices in our approach are a function of both the bidder and the item. Furthermore, they are calculated by solving a bilinear optimization problem, which for the special case of auctions without budget constraints, reduces to a linear optimization problem. The optimal auction mechanism, which is incentive compatible, individually rational and budget feasible has the structure of a VickreyClarke-Groves mechanism, as explained further in subsection 3.2.2 Additionally, we report computational evidence which suggests that the proposed approach:

(a) is numerically tractable for large scale auction design problems, when we use an algorithm based on the Generalized Benders Decomposition to solve the bilinear optimization problem. The notion of tractability used, however, is not the same 
as theoretical tractability (polynomial time solvability) developed in the 1970s (Cook [1971], Karp [1972]). For instance, the Simplex method, which has proven over many decades to be practically efficient is not theoretically efficient. We use exactly this notion of practical efficiency: the ability to solve problems of realistic size relative to the application we address.

(b) leads to improved revenue compared to the classical probabilistic approach, when the true distributions are different from the assumed ones; and

(c) leads to higher revenues, when the correlations in bidders valuations are explicitly modeled.

\subsubsection{Structure of the Chapter and Notation}

The chapter is organized as follows. In Section 3.2, we introduce our approach to Problems (P1) and (P3), and present a characterization of the optimal solution. In Section 3.3, we consider the special case of buyers without budget constraints for Problems (P1), (P3) and derive further insights. In Section 3.4, we address and solve Problem (P2). In Section 3.5, we report computational results. In Section 3.6, we include concluding remarks.

Throughout the rest of the chapter, we denote scalar quantities by non-bold face symbols (e.g., $x \in \mathbb{R}, k \in \mathbb{N}$ ), vectors by boldface symbols (e.g., $\mathbf{x} \in \mathbb{R}^{n}, n>1$. We will use $\mathcal{N}=\{1,2, \ldots, n\}$ to denote the set of buyers and $\mathcal{M}=\{1,2, \ldots, m\}$ to denote the set of items. We will use the index $i$ to denote the $i^{\text {th }}$ buyer and the index $j$ to denote the $j^{\text {th }}$ item.

\subsection{Model and Problem Formulation}

We consider a setting where $n$ buyers, indexed by $i \in \mathcal{N}$, are interested in a set of $m$ items, indexed by $j \in \mathcal{M}$, made available by an auctioneer. Each buyer $i \in \mathcal{N}$ has a valuation $v_{i j}$ associated with each of the items $j \in \mathcal{M}$, which is not known to the auctioneer. Additionally the buyers are also budget constrained with budgets 
$\left\{B_{1}, B_{2}, \ldots, B_{n}\right\}$. In this section, we assume that the budget constraints are public knowledge, and we address the case of uncertainty in budgets in Section 3.4.

Before proceeding further, we introduce the following notation. For each item $j \in \mathcal{M}$, let $\mathbf{v}_{j}=\left(v_{1 j}, v_{2 j}, \ldots, v_{n j}\right) \in \mathbb{R}^{n}$ be the vector of valuations for the $j^{\text {th }}$ item by the $n$ bidders. We let $\mathbf{v}=\left(\mathbf{v}_{1}, \mathbf{v}_{2}, \ldots, \mathbf{v}_{m}\right)$ denote the concatenation of the vectors $\mathbf{v}_{j}, j \in \mathcal{M}$. With a slight abuse of notation, let $\mathbf{v}_{i}=\left(v_{i 1}, v_{i 2}, \ldots, v_{i m}\right)$ be the vector of valuations for the $i^{\text {th }}$ bidder for all items. In the same vein, we let $\mathbf{v}_{-i, j}=\left(v_{1 j}, \ldots, v_{i-1, j}, v_{i+1, j}, \ldots, v_{n j}\right) \in \mathbb{R}^{n-1}$ be the vector of valuations of all bidders except $i$, for item $j, \forall j \in \mathcal{M}$. And let $\mathbf{v}_{-i}=\left(\mathbf{v}_{-i, 1}, \ldots, \mathbf{v}_{-i, m}\right) \in \mathbb{R}^{(n-1) \times m}$ be the concatenation of the vectors $\left(\mathbf{v}_{-i, j}\right)_{j \in \mathcal{M}}$. Finally, we write $\mathbf{v} \in \mathcal{U}$ to denote $\mathbf{v}_{j} \in \mathcal{U}_{j}$, $j \in \mathcal{M}$.

\subsubsection{The Robust Optimization Approach}

We next introduce the concept of worst case optimality, and show how the resulting auction design problem can be formulated as a robust linear optimization problem. As alluded to before, the objective is to maximize the worst case revenue over all valuation vectors $\mathbf{v}$ lying in an uncertainty $\operatorname{set} \mathcal{U}$. We introduce the decision variables $\mathbf{x}^{\mathbf{v}}$ and $\mathbf{p}^{\mathbf{v}}$ that represent the allocation and the payment rules, respectively, for all valuation vectors $\mathbf{v} \in \mathcal{U}$. That is, if the realized valuation vector is $\mathbf{v}$, then we allocate a fraction $x_{i j}^{\mathrm{v}}$ of item $j$ to buyer $i$, and charge a total of $p_{i}^{\mathrm{v}}$ to the $i^{\text {th }}$ buyer. Note that we do not account for payments of buyer $i$ relative to item $j$, but only account for the total payment of buyer $i$.

The allocation and payment rules should be chosen to satisfy the following properties:

(a) Individual Rationality (IR) : This property ensures that the buyers do not derive negative utility by participating in the auction when they bid truthfully.

(b) Budget Feasibility $(B F)$ : This property ensures that each buyer is charged within his budget constraints. 
(c) Incentive Compatibility (IC) : This property ensures that the total utility of the $i^{\text {th }}$ buyer under truthful bidding, which is given by

$$
\begin{aligned}
U\left(\mathbf{v}_{i}, \mathbf{v}_{-i}\right) & =\sum_{j \in \mathcal{M}} v_{i j} \cdot x_{i j}^{\left(\mathbf{v}_{i}, \mathbf{v}_{-i}\right)}-p_{i}^{\left(\mathbf{v}_{i}, \mathbf{v}_{-i}\right)} \\
& \geq \sum_{j \in \mathcal{M}} v_{i j} \cdot x_{i j}^{\left(\mathbf{u}_{i}, \mathbf{v}_{-i}\right)}-p_{i}^{\left(\mathbf{u}_{i}, \mathbf{v}_{-i}\right)} \\
& =U\left(\mathbf{u}_{i}, \mathbf{v}_{-i}\right)
\end{aligned}
$$

is greater than the total utility that Buyer $i$ derives by bidding any other other bid vector $\mathbf{u}_{i}$.

The optimal auction design problem with these properties, leads to the following linear optimization model:

$$
\begin{array}{ll}
Z^{*}(\mathcal{U})=\max \quad & W \\
\text { s.t. } \quad & W-\sum_{i \in \mathcal{N}} p_{i}^{\mathbf{v}} \leq 0, \forall \mathbf{v} \in \mathcal{U} \\
& \sum_{i \in \mathcal{N}} x_{i j}^{\mathbf{v}} \leq 1, \forall j \in \mathcal{M}, \forall \mathbf{v} \in \mathcal{U} \\
& \sum_{j \in \mathcal{M}} v_{i j} \cdot x_{i j}^{\left(\mathbf{u}_{i}, \mathbf{v}_{-i}\right)}-p_{i}^{\left(\mathbf{u}_{i}, \mathbf{v}_{-i}\right)}-\sum_{j \in \mathcal{M}} v_{i j} \cdot x_{i j}^{\left(\mathbf{v}_{i}, \mathbf{v}_{-i}\right)} \\
& +p_{i}^{\left(\mathbf{v}_{i}, \mathbf{v}_{-i}\right)} \leq 0, \forall\left(\mathbf{v}_{i}, \mathbf{v}_{-i}\right) \in \mathcal{U}, \forall\left(\mathbf{u}_{i}, \mathbf{v}_{-i}\right) \in \mathcal{U}, \forall i \in \mathcal{N}, \\
& p_{i}^{\mathbf{v}} \leq B_{i}, \quad \forall i \in \mathcal{N}, \forall \mathbf{v} \in \mathcal{U}, \\
& p_{i}^{\mathbf{v}} \leq \sum_{j \in \mathcal{M}} v_{i j} \cdot x_{i j}^{\mathbf{v}}, \forall i \in \mathcal{N}, \forall \mathbf{v} \in \mathcal{U} \\
& \mathbf{x}^{\mathbf{v}} \geq 0
\end{array}
$$

Constraints (3.1) and (3.2) represent the fact that we are interested in maximizing the worst case revenue. Constraint (3.3) expresses the fact that at most one unit of item $j$ can be assigned to all bidders. Constraints (3.4), (3.5), (3.6) implement the IC, BF and IR properties, respectively. We next present the dual problem of (3.1), by 
using the dual variables $\omega_{\mathbf{v}}, \xi_{j, \mathbf{v}}, \beta_{i, \mathbf{v}_{-i}, \mathbf{v}_{i}, \mathbf{u}_{i}}, \eta_{i, \mathbf{v}}, \theta_{i, \mathbf{v}}$ that correspond to the constraints (3.2)-(3.6), respectively. The dual problem is given by

$$
\begin{aligned}
& \min \sum_{\mathbf{v} \in \mathcal{U}}\left(\sum_{j=1}^{m} \xi_{j, \mathbf{v}}+\sum_{i=1}^{n} \eta_{i, \mathbf{v}} B_{i}\right) \\
& \text { s.t. } \quad \xi_{j,\left(\mathbf{v}_{i}, \mathbf{v}_{-i}\right)}+\sum_{\mathbf{u}_{i}} u_{i j} \cdot \beta_{i, \mathbf{v}_{-i}, \mathbf{u}_{i}, \mathbf{v}_{i}}-v_{i j} \cdot \sum_{\mathbf{u}_{i}} \beta_{i, \mathbf{v}_{-i}, \mathbf{v}_{i}, \mathbf{u}_{i}} \\
& -v_{i j} \cdot \theta_{i,\left(\mathbf{v}_{i}, \mathbf{v}_{-i}\right)} \geq 0, \quad \forall\left(\mathbf{v}_{i}, \mathbf{v}_{-i}\right) \in \mathcal{U} \\
& \sum_{\mathbf{u}_{i}} \beta_{i, \mathbf{v}_{-i}, \mathbf{v}_{i}, \mathbf{u}_{i}}-\sum_{\mathbf{u}_{i}} \beta_{i, \mathbf{v}_{-i}, \mathbf{u}_{i}, \mathbf{v}_{i}}-\omega_{\left(\mathbf{v}_{i}, \mathbf{v}_{-i}\right)}+\eta_{i,\left(\mathbf{v}_{i}, \mathbf{v}_{-i}\right)} \\
& +\theta_{i,\left(\mathbf{v}_{i}, \mathbf{v}_{-i}\right)}=0, \quad \forall\left(\mathbf{v}_{i}, \mathbf{v}_{-i}\right) \in \mathcal{U} \\
& \sum_{\mathbf{v} \in \mathcal{U}} \omega_{\mathbf{v}}=1 \\
& \omega_{\mathbf{v}} \geq 0, \boldsymbol{\xi}_{\mathrm{v}} \geq \mathbf{0}, \beta_{i, \mathrm{v}_{-i}, \mathbf{v}_{i}, \mathbf{u}_{i}} \geq 0, \boldsymbol{\eta}_{\mathrm{v}} \geq \mathbf{0}, \boldsymbol{\theta}_{\mathbf{v}} \geq \mathbf{0} .
\end{aligned}
$$

\subsubsection{A Robust Optimal Mechanism}

In this section, we present a mechanism, that we call ROM (Robust Optimal Mechanism), that constitutes an optimal solution to the optimization problem (3.1). ROM consists of Algorithms ROM.a and ROM.b presented in Figures 3-1 and 3-2, respectively. ROM.a which occurs prior to the realization of a specific valuation vector $\mathbf{v}$, requires as inputs, the uncertainty set $\mathcal{U}$ and the budgets $\left\{B_{i}\right\}_{i \in \mathcal{N}}$. Using these inputs, the following two quantities, which parameterize the auction mechanism in ROM.b, are calculated:

(a) $\left\{r_{i j}^{*}\right\}_{i \in \mathcal{N}, j \in \mathcal{M}}$-We call these quantities reservation prices, with the interpretation that buyer $i$ needs to bid at least $r_{i j}^{*}$ for item $j$, in order to have a possibility of obtaining a non-zero part of item $j$;

(b) $\left\{x_{i j}^{*}\right\}_{i \in \mathcal{N}, j \in \mathcal{M}}-$ We call these quantities nominal allocations, with the interpretation that the final allocation $a_{i j}^{\mathbf{v}}$ of item $j$ to buyer $i$ when the valuation vector $\mathbf{v}$ is realized, is given by

$$
a_{i j}^{\mathbf{v}}=x_{i j}^{*}+y_{i j}^{\mathbf{v}}
$$


where $y_{i j}^{\mathrm{v}}$ is calculated in ROM.b.

In the auction mechanism presented in ROM.b, after the bid vector $\mathbf{v}$ is realized, the algorithm calculates the quantities $y_{i j}^{\mathrm{v}}$ that we call final allocations and the payment $p_{i}^{\mathrm{v}}$ that player $i$ makes.

\section{Discussion: Insights and Implications}

We next present some insights into the auction mechanism presented in Figures 3-1 and 3-2. In particular, (a) we discuss how the bilinear optimization problem used in ROM. $a$ is related to Problem (3.1); and (b) we present a qualitative description of the auction mechanism presented in ROM.b and describe its relationship with the Vickrey-Clarke-Groves (VCG) mechanism.

\section{Structure of ROM.a}

In ROM.a we compute the reservation prices and nominal allocations, introduced above, that parametrize the actual auction mechanism. We compute these quantities by solving a bilinear optimization problem, which we obtain by considering a reduced version of Problem (3.1). In particular, the first two constraints in the inner optimization problem of (3.8) correspond to the constraints (3.3) and (3.5), respectively. The third constraint in the inner optimization problem of (3.8) is a robust optimization constraint and, as we show in the proofs presented in next section, this constraint allows us to capture the incentive compatibility and individual rationality constraints of Problem (3.1).

\section{Structure of ROM.b}

In ROM.b we present the auction mechanism which is carried out when the

bid vector $\mathbf{v}$ is realized. The mechanism calculates the final allocations $y_{i j}^{\mathrm{v}}$ by solving the optimization problem (3.12) where we maximize an affine function of the social welfare determined by the reservation prices $r_{i j}^{*}$ 's. Moreover, the payments $p_{i}^{\mathbf{v}}$ that player $i$ makes are calculated according to Eq. (3.17).

The auction mechanism has the following structural properties: 


\section{Algorithm ROM.a}

Input : Uncertainty set $\mathcal{U}$, and budgets $B_{1}, \ldots, B_{n}$.

Output : Reservation prices $\left\{r_{i j}^{*}\right\}_{i \in \mathcal{N}, j \in \mathcal{M}}$, and nominal allocations $\left\{x_{i j}^{*}\right\}_{i \in \mathcal{N}, j \in \mathcal{M}}$.

1. Compute the worst case valuation vector $\mathbf{z}=\left\{z_{i, j}\right\}_{i \in \mathcal{N}, j \in \mathcal{M}}$ and the nominal allocation vector $\mathrm{x}^{*}=\left\{x_{i j}^{*}\right\}_{i \in \mathcal{N}, j \in \mathcal{M}}$ given by

$$
\left(\mathbf{z}, \mathbf{x}^{*}\right)=\arg \max _{\mathbf{v} \in \mathcal{U}}\left\{\begin{array}{cc}
\max _{\left\{x_{i j}\right\}_{i \in \mathcal{N}, j \in \mathcal{M}}} & \sum_{i \in \mathcal{N}, j \in \mathcal{M}} \sum_{i j} v_{i j} \\
\text { s.t. } & \sum_{i \in \mathcal{N}} x_{i j} \leq 1, \forall j \in \mathcal{M}, \\
& \sum_{j \in \mathcal{M}} x_{i j} \cdot v_{i j} \leq B_{i}, \forall i \in \mathcal{N}, \\
& \sum_{j \in \mathcal{M}} x_{i j} \cdot v_{i j} \leq \sum_{j \in \mathcal{M}} x_{i j} \cdot u_{i j}, \forall \mathbf{u} \in \mathcal{U}, \forall i, \\
\mathbf{x} \geq \mathbf{0} .
\end{array}\right\}
$$

2. Compute $\left(\boldsymbol{\xi}^{*}=\left\{\xi_{j}^{*}\right\}_{j \in \mathcal{M}}, \boldsymbol{\eta}^{*}=\left\{\eta_{i}^{*}\right\}_{i \in \mathcal{N}}, \boldsymbol{\theta}^{*}=\left\{\theta_{i}^{*}\right\}_{i \in \mathcal{N}}\right)$ given by

$$
\left(\boldsymbol{\xi}^{*}, \boldsymbol{\eta}^{*}, \boldsymbol{\theta}^{*}\right)=\arg \left\{\begin{array}{cc}
\min _{\left\{\xi_{j}, \eta_{i}\right\}} & \sum_{\substack{j \in \mathcal{M} \\
\xi_{j}}}+\sum_{i \in \mathcal{N}}\left(\eta_{i} B_{i}+\theta_{i} \sum_{j \in \mathcal{M}} x_{i j}^{*} \tilde{u}_{j}^{i}\right) \\
\text { s.t. } & \xi_{j}+z_{i j} \cdot \eta_{i}+z_{i j} \cdot \theta_{i} \geq z_{i j}, \quad \forall i \in \mathcal{N}, \forall j, \\
& \xi_{j}, \eta_{i}, \theta_{i} \geq 0 .
\end{array}\right\}
$$

where

$$
\tilde{\mathbf{u}}^{i}=\arg \min _{\mathbf{u} \in \mathcal{U}} \sum_{j \in \mathcal{M}} x_{i j}^{*} \cdot u_{i j}, \forall i \in \mathcal{N}
$$

3. Compute the reservation prices $\left\{r_{i j}^{*}\right\}_{i \in \mathcal{N}, j \in \mathcal{M}}$ given by

$$
r_{i j}^{*}=\xi_{j}^{*}+\eta_{i}^{*} \cdot z_{i j}+\theta_{i}^{*} \cdot z_{i j}
$$

Figure 3-1: Calculation of the reservation prices and the nominal allocations. 
Algorithm ROM.b

Input : Bid vector $\mathbf{v}=\left\{v_{i j}\right\}_{i \in \mathcal{N}, j \in \mathcal{M}},\left\{r_{i j}^{*}\right\}_{i \in \mathcal{N}, j \in \mathcal{M}},\left\{x_{i j}^{*}\right\}_{i \in \mathcal{N}, j \in \mathcal{M}}$.

Output : Allocation vector $\left\{a_{i j}^{\mathrm{v}}\right\}_{i \in \mathcal{N}, j \in \mathcal{M}}$ and the payments $\left\{p_{k}^{\vee}\right\}_{k \in \mathcal{N}}$.

1. Check if $\mathrm{v} \in \mathcal{U}$. If $\mathrm{v} \notin \mathcal{U}$ then do not allocate anything to anybody and charge zero.

2. Calculate the adapted allocations $y_{i j}^{\mathrm{v}}$ and temporary quantities $y_{i j, k}^{\mathrm{v}}$ given by

$$
\begin{aligned}
\left\{y_{i j}^{\mathbf{v}}\right\}_{i \in \mathcal{N}, j \in \mathcal{M}} & =\arg \max _{\mathbf{y} \in \mathcal{P}} \sum_{i \in \mathcal{N}} \sum_{j \in \mathcal{M}} y_{i j} \cdot\left(v_{i j}-r_{i j}^{*}\right), \\
\left\{y_{i j, k}^{\mathbf{v}-k}\right\}_{i \in \mathcal{N} \backslash\{k\}, j \in \mathcal{M}} & =\arg \max _{\mathbf{y} \in \mathcal{Q}_{k}} \sum_{i \in \mathcal{N} \backslash\{k\}} \sum_{j \in \mathcal{M}} y_{i j} \cdot\left(v_{i j}-r_{i j}^{*}\right),
\end{aligned}
$$

where the sets $\left(\mathcal{P}, \mathcal{Q}_{1}, \ldots, \mathcal{Q}_{n}\right)$ are given by

$$
\begin{gathered}
\mathcal{P}=\left\{\begin{array}{c|cc}
\sum_{i \in \mathcal{N}} y_{i j} \leq 1-\sum_{i \in \mathcal{N}} x_{i j}^{*}, & \forall j \in \mathcal{M} \\
\sum_{j}^{n \times m} y_{i j} \cdot u_{i j} \leq B_{i}-\sum_{j \in \mathcal{M}} x_{i j}^{*} \cdot r_{i j}^{*} & \\
+\sum_{j \in \mathcal{M}} x_{k j}^{*} \cdot \theta_{i}^{*} \cdot \tilde{u}_{j}^{i}, & \forall \mathbf{u} \in \mathcal{U}, \forall i \in \mathcal{N}
\end{array}\right\} \\
\mathcal{Q}_{k}=\left\{\begin{array}{c|c|}
\sum_{i \in \mathcal{N} \backslash\{k\}} y_{i j} \leq 1-\sum_{i \in \mathcal{N}} x_{i j}^{*}, & \forall j \in \mathcal{M}, \\
\sum_{j \in \mathcal{M}} y_{i j} \cdot u_{i j} \leq B_{i}-\sum_{j \in \mathcal{M}} x_{i j}^{*} \cdot r_{i j}^{*}, & \forall \mathbf{u} \in \mathcal{U}, \forall i \in \mathcal{N} \backslash\{k\}
\end{array}\right\} .
\end{gathered}
$$

3. Compute the allocation vector $\left\{a_{i j}^{\mathrm{v}}\right\}_{i \in \mathcal{N}, j \in \mathcal{M}}$ and the payments $\left\{p_{k}^{\mathrm{v}}\right\}_{k=1, \ldots, n}$ as follows

$$
\begin{aligned}
a_{i j}^{\mathrm{v}}= & y_{i j}^{\mathrm{v}}+x_{i j}^{*}, \\
p_{k}^{\mathrm{v}}= & \sum_{j \in \mathcal{M}} y_{k j}^{\mathrm{v}} \cdot r_{k j}^{*}+\sum_{j \in \mathcal{M}} x_{k j}^{*} \cdot r_{k j}^{*}-\sum_{j \in \mathcal{M}} x_{k j}^{*} \cdot \theta_{k}^{*} \cdot \tilde{u}_{j}^{k} \\
& +\sum_{i \in \mathcal{N} \backslash\{k\}} \sum_{j \in \mathcal{M}} y_{i j, k}^{\mathbf{v}-k} \cdot\left(v_{i j}-r_{i j}^{*}\right)-\sum_{i \in \mathcal{N} \backslash\{k\}} \sum_{j \in \mathcal{M}} y_{i j}^{\mathbf{v}} \cdot\left(v_{i j}-r_{i j}^{*}\right), \forall k \in \mathcal{N} .
\end{aligned}
$$

Figure 3-2: Calculation of adapted allocations and payments. 
a) The allocation rule has a structure very similar to that of VCG auction mechanisms, where the items are allocated to a set of bidders so as to maximize a certain social welfare function. In ROM.b, the social welfare function is given by

$$
\max _{\mathbf{y} \in \mathcal{P}} \sum_{i \in \mathcal{N}} \sum_{j \in \mathcal{M}} y_{i j} \cdot\left(v_{i j}-r_{i j}^{*}\right)
$$

which is parameterized by the reservation prices $\left\{r_{i j}^{*}\right\}$.

b) The payment rule given by Eq. (3.17) is again similar to that of a VCG auction, where each bidder is charged the lowest amount (opportunity cost) it could have bid to still be part of the winning allocation, all other agents bids remaining the same. Moreover, as we see in the next section, this payment rule leads to incentive compatibility as well as to the second price structure in the absence of budgets as shown in subsection 3.3.1.

c) Unlike the Myerson auction where the reservation prices do not depend on the item, the reservation prices in $R O M$ are a function of both the bidder and the item. The existence of budgets leads to this dependence, and we see in subsection 3.3.1, in the absence of budgets, the reservation prices only depend on the bidder.

We next present other structural properties of $R O M$ and their corresponding proofs.

\section{Properties of $R O M$}

In the remaining part of this section, we show that ROM is incentive compatible, budget feasible, individually rational and worst case optimal. The following proposition helps us in preparing the ground.

Proposition 14. The quantities $\left(\mathbf{z}, \mathbf{x}^{*}, \boldsymbol{\xi}^{*}, \boldsymbol{\eta}^{*}, \boldsymbol{\theta}^{*}, \mathbf{r}^{*}\right)$ computed in $\mathrm{ROM}$, satisfy the 
following relations:

$$
\begin{aligned}
\sum_{i \in \mathcal{N}} x_{i j}^{*} & \leq 1, \forall j \in \mathcal{M}, \\
\sum_{j \in \mathcal{M}} x_{i j}^{*} \cdot z_{i j} & \leq B_{i}, \forall i \in \mathcal{N}, \\
\sum_{j \in \mathcal{M}} x_{i j}^{*} \cdot z_{i j} & \leq \sum_{j \in \mathcal{M}} x_{i j}^{*} \cdot u_{i j}, \forall \mathbf{u} \in \mathcal{U}, \forall i \in \mathcal{N}, \\
x_{i j}^{*} \cdot r_{i j}^{*} & =x_{i j}^{*} \cdot z_{i j}, \forall i \in \mathcal{N}, \forall j \in \mathcal{M}, \\
\sum_{j \in \mathcal{M}} x_{i j}^{*} \cdot r_{i j}^{*} & \leq B_{i}, \forall i \in \mathcal{N}, \\
\sum_{i \in \mathcal{N}} \sum_{j \in \mathcal{M}} x_{i j}^{*} \cdot r_{i j}^{*} & =\sum_{j \in \mathcal{M}} \xi_{j}^{*}+\sum_{i \in \mathcal{N}}\left(\eta_{i}^{*} B_{i}+\sum_{j \in \mathcal{M}} x_{i j}^{*} \cdot \theta_{i}^{*} \cdot \tilde{u}_{j}^{i}\right) .
\end{aligned}
$$

Proof. The relations (3.18)-(3.20) follow from the definition of $\left(\mathbf{z}, \mathbf{x}^{*}\right)$ in (3.8). Indeed, fixing $\mathbf{z}$, consider the optimization problem

$$
\begin{array}{cc}
\max _{\left\{x_{i j}\right\}_{i \in \mathcal{N}, j \in \mathcal{M}}} & \sum_{i \in \mathcal{N},} \sum_{j \in \mathcal{M}} x_{i j} z_{i j} \\
\text { s.t. } & \sum_{i \in \mathcal{N}} x_{i j} \leq 1, \forall j \in \mathcal{M}, \\
& \sum_{j \in \mathcal{M}} x_{i j} \cdot z_{i j} \leq B_{i}, \forall i \in \mathcal{N}, \\
& \sum_{j \in \mathcal{M}} x_{i j} \cdot z_{i j} \leq \sum_{j \in \mathcal{M}} x_{i j} \cdot u_{i j}, \forall \mathbf{u} \in \mathcal{U}, \forall i \in \mathcal{N} .
\end{array}
$$

By definition $\mathrm{x}^{*}$ is an optimal solution to (3.24), and this observation directly implies (3.18)-(3.20). Now consider another optimization problem

$$
\begin{array}{cc}
\max _{\left\{x_{i j}\right\}_{i \in \mathcal{N}, j \in \mathcal{M}}} & \sum_{i \in \mathcal{N},} \sum_{j \in \mathcal{M}} x_{i j} z_{i j} \\
\text { s.t. } & \sum_{i \in \mathcal{N}} x_{i j} \leq 1, \forall j \in \mathcal{M}, \\
& \sum_{j \in \mathcal{M}} x_{i j} \cdot z_{i j} \leq B_{i}, \forall i \in \mathcal{N}, \\
& \sum_{j \in \mathcal{M}} x_{i j} \cdot z_{i j} \leq \sum_{j \in \mathcal{M}} x_{i j}^{*} \cdot u_{i j}, \forall \mathbf{u} \in \mathcal{U}, \forall i \in \mathcal{N},
\end{array}
$$


which differs from (3.24) only in the last family of constraints. Let $\overline{\mathbf{x}}$ be the optimal solution of (3.25). Since $\mathrm{x}^{*}$ is feasible to (3.24), $\mathrm{x}^{*}$ is also feasible to (3.25). This implies that

$$
\sum_{i \in \mathcal{N},} \sum_{j \in \mathcal{M}} \bar{x}_{i j} z_{i j} \geq \sum_{i \in \mathcal{N}, j \in \mathcal{M}} \sum_{i j}^{*} z_{i j}
$$

We also have the following constraints that $\overline{\mathbf{x}}$ satisfies

$$
\sum_{j \in \mathcal{M}} \bar{x}_{i j} \cdot z_{i j} \leq \sum_{j \in \mathcal{M}} x_{i j}^{*} \cdot u_{i j}, \forall \mathbf{u} \in \mathcal{U}, \forall i \in \mathcal{N}
$$

In particular, since $\mathbf{z} \in \mathcal{U}$, we have

$$
\sum_{j \in \mathcal{M}} \ddot{x}_{i j} \cdot z_{i j} \leq \sum_{j \in \mathcal{M}} x_{i j}^{*} \cdot z_{i j}, \forall i \in \mathcal{N}
$$

From (3.20) and (3.27), we have

$$
\sum_{i \in \mathcal{N},} \sum_{j \in \mathcal{M}} \bar{x}_{i j} z_{i j}=\sum_{i \in \mathcal{N},} \sum_{j \in \mathcal{M}} x_{i j}^{*} z_{i j}
$$

which implies that $\mathrm{x}^{*}$ is an optimal solution to (3.25). Now consider an equivalent formulation of (3.25) given by

$$
\begin{array}{cc}
\max _{\left\{x_{i j}\right\}_{i \in \mathcal{N}, j \in \mathcal{M}}} & \sum_{i \in \mathcal{N},} \sum_{j \in \mathcal{M}} x_{i j} z_{i j} \\
\text { s.t. } & \sum_{i \in \mathcal{N}} x_{i j} \leq 1, \forall j \in \mathcal{M}, \\
& \sum_{j \in \mathcal{M}} x_{i j} \cdot z_{i j} \leq B_{i}, \forall i \in \mathcal{N}, \\
& \sum_{j \in \mathcal{M}} x_{i j} \cdot z_{i j} \leq \sum_{j \in \mathcal{M}} x_{i j}^{*} \cdot \tilde{u}_{j}^{i}, \forall i \in \mathcal{N},
\end{array}
$$

where $\tilde{\mathbf{u}}^{i}$ is as defined in (3.10). It is clear that (3.29) is equivalent to (3.25), thus indicating that $\mathbf{x}^{*}$ is an optimal solution of (3.29). The dual of Problem (3.29) is 
given by

$$
\begin{array}{cc}
\min _{\left\{\xi_{j}, \eta_{i}\right\}} & \sum_{j \in \mathcal{M}} \xi_{j}+\sum_{i \in \mathcal{N}}\left(\eta_{i} B_{i}+\theta_{i} \sum_{j \in \mathcal{M}} x_{i j}^{*} \tilde{u}_{j}^{i}\right) \\
\text { s.t. } & \xi_{j}+z_{i j} \cdot \eta_{i}+z_{i j} \cdot \theta_{i} \geq z_{i j}, \quad \forall i \in \mathcal{N}, \forall j \in \mathcal{M}, \\
\xi_{j}, \eta_{i}, \theta_{i} \geq 0
\end{array}
$$

where $\xi_{j}, \eta_{i}, \theta_{i}$ correspond to the first, second and third family of constraints, respectively. Let $\left(\boldsymbol{\xi}^{*}, \boldsymbol{\eta}^{*}, \boldsymbol{\theta}^{*}\right)$ be the dual solution corresponding to the primal solution $\mathbf{x}^{*}$. Then by complementary slackness, we have the following relations

$$
\begin{aligned}
\xi_{j}^{*} \cdot \sum_{i \in \mathcal{N}} x_{i j}^{*} & =\xi_{j}^{*}, \forall j \in \mathcal{M}, \\
\eta_{i}^{*} \cdot \sum_{j \in \mathcal{M}} x_{i j}^{*} \cdot z_{i j} & =\eta_{i}^{*} \cdot B_{i}, \forall i \in \mathcal{N}, \\
\theta_{i}^{*} \cdot \sum_{j \in \mathcal{M}} x_{i j}^{*} \cdot z_{i j} & =\theta_{i}^{*} \cdot \sum_{j \in \mathcal{M}} x_{i j}^{*} \cdot \tilde{u}_{j}^{i}, \quad i \in \mathcal{N}, \\
x_{i j}^{*} \cdot\left(\xi_{j}+z_{i j} \cdot \eta_{i}+z_{i j} \cdot \theta_{i}\right) & =x_{i j}^{*} \cdot z_{i j}, \quad \forall i \in \mathcal{N}, \forall j \in \mathcal{M} .
\end{aligned}
$$

Also, given that $\mathbf{x}^{*}$ is optimal to (3.29), we have by strong duality

$$
\sum_{i \in \mathcal{N},} \sum_{j \in \mathcal{M}} x_{i j}^{*} z_{i j}=\sum_{j \in \mathcal{M}} \xi_{j}^{*}+\sum_{i \in \mathcal{N}}\left(\eta_{i}^{*} B_{i}+\theta_{i}^{*} \sum_{j \in \mathcal{M}} x_{i j}^{*} \tilde{u}_{j}^{i}\right)
$$

Now (3.21) and (3.23) follow from (3.31) and (3.32). Finally (3.29) follows from (3.25), (3.27), and the fact that $\boldsymbol{\theta}^{*} \geq \mathbf{0}$. This concludes the proof.

Proposition 15. [Incentive Compatibility] The total allocation vector $\left\{a_{i j}^{\mathbf{v}}\right\}_{i, j}$ and the payments $\left\{p_{i}^{\mathbf{v}}\right\}_{i \in \mathcal{N}}$ satisfy (3.4), that is, the overall mechanism is incentive compatible.

Proof. Consider buyer $k$ with a true valuation vector $\left\{v_{k j}\right\}_{j \in \mathcal{M}}$. By bidding $\left\{v_{k j}^{\prime}\right\}_{j \in \mathcal{M}}$ ' buyer $k$ is allocated a fraction $y_{k j}^{\left(v_{k}^{\prime}, v_{-k}\right)}$ of item $j$. From (3.12) and the fact that $\mathcal{P}$ does not depend on any specific valuation vector, we have 


$$
\left\{y_{i j}^{\left(\mathbf{v}_{k}^{\prime}, \mathbf{v}_{-k}\right)}\right\}_{i \in \mathcal{N}, j \in \mathcal{M}} \in \mathcal{P}
$$

Again from (3.12) we have

$$
\left\{y_{i j}^{\left(\mathbf{v}_{k}, \mathbf{v}_{-k}\right)}\right\}_{i \in \mathcal{N}, j \in \mathcal{M}}=\arg \max _{\mathbf{y} \in \mathcal{P}} \sum_{i \in \mathcal{N}} \sum_{j \in \mathcal{M}} y_{i j} \cdot\left(v_{i j}-r_{i j}^{*}\right)
$$

which along with (3.33) implies that

$$
\sum_{i \in \mathcal{N}} \sum_{j \in \mathcal{M}} y_{i j}^{\left(\mathbf{v}_{k}, \mathbf{v}_{-k}\right)} \cdot\left(v_{i j}-r_{i j}^{*}\right) \geq \sum_{i \in \mathcal{N}} \sum_{j \in \mathcal{M}} y_{i j}^{\left(\mathbf{v}_{k}^{\prime}, \mathbf{v}_{-k}\right)} \cdot\left(v_{i j}-r_{i j}^{*}\right)
$$

Now let $U\left(\mathbf{u}, \mathbf{v}_{-k}\right)$ denote the utility of buyer $k$ when he bids $\mathbf{u}=\left(u_{1}, \ldots, u_{m}\right)$ while the other buyers bid $\mathbf{v}_{-k}$. Then we have

$$
\begin{aligned}
U\left(\mathbf{v}_{k}, \mathbf{v}_{-k}\right)= & \sum_{j \in \mathcal{M}} a_{k j}^{\left(\mathbf{v}_{k}, \mathbf{v}_{-k}\right)} \cdot v_{k j}-p_{k}^{\left(\mathbf{v}_{k}, \mathbf{v}_{-k}\right)} \\
= & \sum_{j \in \mathcal{M}} y_{k j}^{\left(\mathbf{v}_{k}, \mathbf{v}_{-k}\right)} \cdot v_{k j}+\sum_{j \in \mathcal{M}} x_{k j}^{*} \cdot v_{k j} \\
& -\sum_{j \in \mathcal{M}} y_{k j}^{\left(\mathbf{v}_{k}, \mathbf{v}_{-k}\right)} \cdot r_{k j}^{*}-\sum_{j \in \mathcal{M}} x_{k j}^{*} \cdot r_{k j}^{*}+\sum_{j \in \mathcal{M}} x_{k j}^{*} \cdot \theta_{k}^{*} \cdot \tilde{u}_{j}^{k} \\
& -\sum_{i \in \mathcal{M} \backslash\{k\}} \sum_{j \in \mathcal{M}} y_{i j, k}^{\mathbf{v}_{-k}}\left(v_{i j}-r_{i j}^{*}\right)+\sum_{i \in \mathcal{M} \backslash\{k\}} \sum_{j \in \mathcal{M}} y_{i j}^{\left(\mathbf{v}_{k}, \mathbf{v}_{-k}\right)}\left(v_{i j}-r_{i j}^{*}\right)(3 \\
= & \sum_{i \in \mathcal{N}} \sum_{j \in \mathcal{M}} y_{i j}^{\left(\mathbf{v}_{k}, \mathbf{v}_{-k}\right)}\left(v_{i j}-r_{i j}^{*}\right)-\sum_{i \in \mathcal{N} \backslash\{k\}} \sum_{j \in \mathcal{M}} y_{i j, k}^{\mathbf{v}_{-k}}\left(v_{i j}-r_{i j}^{*}\right) \\
& +\sum_{j \in \mathcal{M}} x_{k j}^{*} \cdot v_{k j}-\sum_{j \in \mathcal{M}} x_{k j}^{*} \cdot r_{k j}^{*}+\sum_{j \in \mathcal{M}} x_{k j}^{*} \cdot \theta_{k}^{*} \cdot \tilde{u}_{j}^{k} \\
\geq & \sum_{i \in \mathcal{N}} \sum_{j \in \mathcal{M}} y_{i j}^{\left(\mathbf{v}_{k}^{\prime}, \mathbf{v}_{-k}\right)} \cdot\left(v_{i j}-r_{i j}^{*}\right)-\sum_{i \in \mathcal{M} \backslash\{k\}} \sum_{j \in \mathcal{M}} y_{i j, k}^{\mathbf{v}_{-k}} \cdot\left(v_{i j}-r_{i j}^{*}\right) \\
& +\sum_{j \in \mathcal{M}} x_{k j}^{*} \cdot v_{k j}-\sum_{j \in \mathcal{M}} x_{k j}^{*} \cdot r_{k j}^{*}+\sum_{j \in \mathcal{M}} x_{k j}^{*} \cdot \theta_{k}^{*} \cdot \tilde{u}_{j}^{k}
\end{aligned}
$$




$$
\begin{aligned}
= & \sum_{j \in \mathcal{M}} y_{k j}^{\left(\mathbf{v}_{k}^{\prime}, \mathbf{v}_{-k}\right)} \cdot v_{k j}+\sum_{j \in \mathcal{M}} x_{k j}^{*} \cdot v_{k j}-\sum_{j \in \mathcal{M}} y_{k j}^{\left(\mathbf{v}_{k}^{\prime}, \mathbf{v}_{-k}\right)} \cdot r_{k j}^{*}-\sum_{j \in \mathcal{M}} x_{k j}^{*} \cdot r_{k j}^{*} \\
& -\sum_{i \in \mathcal{N} \backslash\{k\}} \sum_{j \in \mathcal{M}} y_{i j, k}^{\mathbf{v}_{-k}} \cdot\left(v_{i j}-r_{i j}^{*}\right)+\sum_{i \in \mathcal{N} \backslash\{k\}} \sum_{j \in \mathcal{M}} y_{i j}^{\left(\mathbf{v}_{k}, \mathbf{v}_{-k}\right)} \cdot\left(v_{i j}-r_{i j}^{*}\right)(3.38) \\
= & U\left(\mathbf{v}_{k}^{\prime}, \mathbf{v}_{-k}\right),
\end{aligned}
$$

where in (3.35) we combine the first, third and the last terms to obtain the first term in (3.36). (3.37) follows from (3.34) and (3.38) follows by rearranging terms in (3.37). This concludes the proof.

Proposition 16. [Budget Feasibility] The total allocation vector $\left\{a_{i j}^{\mathbf{v}}\right\}_{i \in \mathcal{N}, j \in \mathcal{M}}$ and the payments $\left\{p_{i}^{\vee}\right\}_{i \in \mathcal{N}}$ satisfy (3.5), that is, the overall mechanism is budget feasible.

Proof. The payment of buyer $k$ is given by

$$
\begin{aligned}
p_{k}^{\mathbf{v}}= & \sum_{j \in \mathcal{M}} y_{k j}^{\mathbf{v}} \cdot r_{k j}^{*}+\sum_{j \in \mathcal{M}} x_{k j}^{*} \cdot r_{k j}^{*}-\sum_{j \in \mathcal{M}} x_{k j}^{*} \cdot \theta_{k}^{*} \cdot \tilde{u}_{j}^{k} \\
& +\sum_{i \in \mathcal{N} \backslash\{k\}} \sum_{j \in \mathcal{M}} y_{i j, k}^{\mathbf{v}-k} \cdot\left(v_{i j}-r_{i j}^{*}\right)-\sum_{i \in \mathcal{M} \backslash\{k\}} \sum_{j \in \mathcal{M}} y_{i j}^{\mathbf{v}} \cdot\left(v_{i j}-r_{i j}^{*}\right) \\
= & \sum_{j \in \mathcal{M}} y_{k j}^{\mathbf{v}} \cdot v_{k j}+\sum_{j \in \mathcal{M}} x_{k j}^{*} \cdot r_{k j}^{*}-\sum_{j \in \mathcal{M}} x_{k j}^{*} \cdot \theta_{k}^{*} \cdot \tilde{u}_{j}^{k} \\
& +\sum_{i \in \mathcal{N} \backslash\{k\}} \sum_{j \in \mathcal{M}} y_{i j, k}^{\mathbf{v}-k} \cdot\left(v_{i j}-r_{i j}^{*}\right)-\sum_{i \in \mathcal{N}} \sum_{j \in \mathcal{M}} y_{i j}^{\mathbf{v}} \cdot\left(v_{i j}-r_{i j}^{*}\right)
\end{aligned}
$$

where (3.40) follows by adding and substracting the term $\sum_{j \in \mathcal{M}} y_{k j}^{v} \cdot v_{k j}$ from (3.39). Now from (3.13), we have

$$
\left\{y_{i j, k}^{\mathbf{v}_{-k}}\right\}_{i \in \mathcal{N} \backslash\{k\}, j \in \mathcal{M}} \in \mathcal{Q}_{k}
$$


which implies that

$$
\begin{aligned}
\sum_{i \in \mathcal{M} \backslash k\}} y_{i j, k}^{\mathbf{v}-k} & \leq 1-\sum_{i \in \mathcal{N}} x_{i j}^{*}, \quad \forall j \in \mathcal{M} \\
\sum_{j \in \mathcal{M}} y_{i j, k}^{\mathbf{v}-k} \cdot u_{i j} & \leq B_{i}-\sum_{j \in \mathcal{M}} x_{i j}^{*} \cdot r_{i j}^{*}, \quad \forall \mathbf{u} \in \mathcal{U}, \forall i \in \mathcal{N} \backslash\{k\} .
\end{aligned}
$$

Thus, by constructing an allocation vector $\hat{\mathbf{y}}$ such that

$$
\hat{y}_{i j}= \begin{cases}y_{i j, k}^{\mathbf{v}_{-k}}, & \forall i \in \mathcal{N} \backslash\{k\}, \forall j \in \mathcal{M}, \\ 0, & i=k, \forall j \in \mathcal{M},\end{cases}
$$

we have from (3.41)

$$
\begin{aligned}
\sum_{i \in \mathcal{N}} \hat{y}_{i j} & =\sum_{i \in \mathcal{N} \backslash\{k\}} y_{i j, k}^{\mathbf{v}-k} \\
& \leq 1-\sum_{i \in \mathcal{N}} x_{i j}^{*}, \quad \forall j \in \mathcal{M} \\
\sum_{j \in \mathcal{M}} \hat{y}_{i j} \cdot u_{i j} & =\sum_{j \in \mathcal{M}} y_{i j, k}^{\mathbf{v}-k} \cdot u_{i j} \\
& \leq B_{i}-\sum_{j \in \mathcal{M}} x_{i j}^{*} \cdot r_{i j}^{*}, \quad \forall \mathbf{u} \in \mathcal{U}, \forall i \in \mathcal{N} \backslash\{k\}
\end{aligned}
$$

Also we have

$$
\sum_{j \in \mathcal{M}} \hat{y}_{k j} \cdot u_{k j}=0 \leq B_{k}-\sum_{j \in \mathcal{M}} x_{k j}^{*} \cdot r_{k j}^{*}
$$

where the second part follows from (3.29) from Proposition 14. (3.42) and (3.43) imply that $\hat{\mathbf{y}} \in \mathcal{P}$ which implies that

$$
\begin{aligned}
\sum_{i \in \mathcal{N}} \sum_{j \in \mathcal{M}} y_{i j}^{\mathbf{v}} \cdot\left(v_{i j}-r_{i j}^{*}\right) & \geq \sum_{i \in \mathcal{N}} \sum_{j \in \mathcal{M}} \hat{y_{i j}} \cdot\left(v_{i j}-r_{i j}^{*}\right) \\
& =\sum_{i \in \mathcal{N} \backslash\{k\}} \sum_{j \in \mathcal{M}} y_{i j, k}^{\mathbf{v}-k} \cdot\left(v_{i j}-r_{i j}^{*}\right)
\end{aligned}
$$


Thus, from (3.40) and (3.44), we have

$$
\begin{aligned}
p_{k}^{\mathrm{v}} & \leq \sum_{j \in \mathcal{M}} y_{k j}^{\mathrm{v}} \cdot v_{k j}+\sum_{j \in \mathcal{M}} x_{k j}^{*} \cdot r_{k j}^{*}-\sum_{j \in \mathcal{M}} x_{k j}^{*} \cdot \theta_{k}^{*} \cdot \tilde{u}_{j}^{k} \\
& \leq B_{k}
\end{aligned}
$$

where the last inequality holds because $\mathbf{y}^{\mathbf{v}} \in \mathcal{P}$. This concludes the proof.

Proposition 17. [Individually Rationality] The total allocation vector $\left\{a_{i j}^{\mathbf{v}}\right\}_{i, j}$ and the payments $\left\{p_{i}^{\mathbf{v}}\right\}_{i \in \mathcal{N}}$ satisfy (3.6), that is, the overall mechanism is individually rational.

Proof. As before, let $U\left(\mathbf{u}, \mathbf{v}_{-k}\right)$ be the utility of buyer $k$ when he bids $\mathbf{u}$. Then from (3.36), we have

$$
\begin{aligned}
U\left(\mathbf{v}_{k}, \mathbf{v}_{-k}\right)= & \sum_{i \in \mathcal{N}} \sum_{j \in \mathcal{M}} y_{i j}^{\left(\mathbf{v}_{k}, \mathbf{v}_{-k}\right)} \cdot\left(v_{i j}-r_{i j}^{*}\right)-\sum_{i \in \mathcal{M}\{k\}} \sum_{j \in \mathcal{M}} y_{i j, k}^{\mathbf{v}_{-k}} \cdot\left(v_{i j}-r_{i j}^{*}\right) \\
& +\sum_{j \in \mathcal{M}} x_{k j}^{*} \cdot v_{k j}-\sum_{j \in \mathcal{M}} x_{k j}^{*} \cdot r_{k j}^{*}+\sum_{j \in \mathcal{M}} x_{k j}^{*} \cdot \theta_{k}^{*} \cdot \tilde{u}_{j}^{k} \\
\geq & \sum_{j \in \mathcal{M}} x_{k j}^{*} \cdot v_{k j}-\sum_{j \in \mathcal{M}} x_{k j}^{*} \cdot r_{k j}^{*}+\sum_{j \in \mathcal{M}} x_{k j}^{*} \cdot \theta_{k}^{*} \cdot \tilde{u}_{j}^{k}
\end{aligned}
$$

where (3.45) follows from (3.44). Now from (3.20) and (3.21) of Proposition 14, we have

$$
\begin{aligned}
\sum_{j \in \mathcal{M}} x_{k j}^{*} \cdot v_{k j} & \geq \sum_{j \in \mathcal{M}} x_{k j}^{*} \cdot z_{k j} \\
& =\sum_{j \in \mathcal{M}} x_{k j}^{*} \cdot r_{k j}^{*} \\
& \geq \sum_{j \in \mathcal{M}} x_{k j}^{*} \cdot r_{k j}^{*}-\sum_{j \in \mathcal{M}} x_{k j}^{*} \cdot \theta_{k}^{*} \cdot \tilde{u}_{j}^{k}
\end{aligned}
$$

where the last inequality follows from the fact that $x_{k j}^{*}, \theta_{k}^{*}, \tilde{u}_{k j}$ are all non-negative. Finally this implies that

$$
U\left(\mathbf{v}_{k}, \mathbf{v}_{-k}\right) \geq 0
$$

which concludes the proof. 
Proposition 18. [Worst case nature of $\mathbf{z}$ ] The valuation vector $\mathbf{z}$ achieves the worst case revenue of $\mathrm{ROM}$, that is, the allocations $\left\{a_{i j}^{\mathrm{v}}\right\}_{i \in \mathcal{N}, j \in \mathcal{M}}$ and the payments $\left\{p_{i}^{\mathbf{v}}\right\}_{i \in \mathcal{N}}$ satisfy (3.2).

Proof. Consider any valuation vector $\mathbf{v}$ and consider the payments received by the auctioneer. The total revenue is given by

$$
\begin{aligned}
\sum_{k \in \mathcal{N}} p_{k}^{\mathbf{v}}=\sum_{k \in \mathcal{N}} & {\left[\sum_{j \in \mathcal{M}} y_{k j}^{\mathbf{v}} \cdot r_{k j}^{*}+\sum_{j \in \mathcal{M}} x_{k j}^{*} \cdot r_{k j}^{*}-\sum_{j \in \mathcal{M}} x_{k j}^{*} \cdot \theta_{k}^{*} \cdot \tilde{u}_{j}^{k}\right.} \\
& \left.+\sum_{i \in \mathcal{M} \backslash\{\}\}} \sum_{j \in \mathcal{M}} y_{i j, k}^{\mathbf{v}-k} \cdot\left(v_{i j}-r_{i j}^{*}\right)-\sum_{i \in \mathcal{N} \backslash\{k\}} \sum_{j \in \mathcal{M}} y_{i j}^{\mathbf{v}} \cdot\left(v_{i j}-r_{i j}^{*}\right)\right] .
\end{aligned}
$$

Now consider the vector $\hat{\mathbf{y}}$ given by

$$
\hat{y}_{i j}=y_{i j}^{\mathbf{v}}, \forall i \in \mathcal{N} \backslash\{k\}, \forall j \in \mathcal{M}
$$

Since $\mathbf{y}^{\mathbf{v}} \in \mathcal{P}$, it is easy to verify that

$$
\hat{\mathbf{y}} \in \mathcal{Q}_{k} .
$$

Thus, from (3.13) we have that

$$
\begin{aligned}
\sum_{i \in \mathcal{N} \backslash\{k\}} \sum_{j \in \mathcal{M}} y_{i j, k}^{\mathbf{v}-k} \cdot\left(v_{i j}-r_{i j}^{*}\right) & \geq \sum_{i \in \mathcal{N} \backslash\{k\}} \sum_{j \in \mathcal{M}} \hat{y}_{i j} \cdot\left(v_{i j}-r_{i j}^{*}\right) \\
& =\sum_{i \in \mathcal{N} \backslash\{k\}} \sum_{j \in \mathcal{M}} y_{i j}^{\mathbf{v}} \cdot\left(v_{i j}-r_{i j}^{*}\right)
\end{aligned}
$$

Also from the non-negativity of $y_{k j}^{\mathrm{v}}, r_{k j}^{*}$, we have

$$
\sum_{j \in \mathcal{M}} y_{k j}^{v} \cdot r_{k j}^{*} \geq 0
$$


From (3.46) and (3.47), we have

$$
\sum_{k=1}^{n} p_{k}^{\mathrm{v}} \geq \sum_{k=1}^{n} \sum_{j=1}^{m} x_{k j}^{*} \cdot r_{k j}^{*}-\sum_{j \in \mathcal{M}} x_{k j}^{*} \cdot \theta_{k}^{*} \cdot \tilde{u}_{j}^{k}
$$

To finish the proof, we observe that

$$
\begin{aligned}
y_{i j}^{\mathbf{z}} & =0, \forall i \in \mathcal{N}, \forall j \in \mathcal{M}, \\
y_{i j, k}^{\mathbf{z}_{-k}} & =0, \forall i \in \mathcal{N} \backslash\{k\}, \forall j \in \mathcal{M}, \forall k \in \mathcal{N},
\end{aligned}
$$

which implies that

$$
\sum_{k=1}^{n} p_{k}^{\mathbf{z}}=\sum_{k=1}^{n} \sum_{j=1}^{m} x_{k j}^{*} \cdot r_{k j}^{*}-\sum_{j \in \mathcal{M}} x_{k j}^{*} \cdot \theta_{k}^{*} \cdot \tilde{u}_{j}^{k}
$$

This concludes the proof.

In Propositions 15,16,17, and 18, we have shown that ROM is incentive compatible, budget feasible, individually rational, and achieves its worst case revenue for the valuation vector $\mathbf{z}$. We next show that ROM is an optimal mechanism.

Theorem 19. [Main Result] ROM is an optimal mechanism for

(a) finite discrete uncertainty sets,

(b) bounded general uncertainty sets.

Proof.

(a) We first note that for finite discrete uncertainty sets, the problems (3.1) and (3.7) are linear optimization problems with a finite number of variables and constraints, and thus they satisfy strong duality. The main idea of the proof then is to construct feasible solutions to the problems (3.1) and (3.7) with the same value for the objective solutions and then appeal to strong duality. We begin by constructing a dual-feasible solution. Let

$$
\left(\mathbf{z}, \mathbf{x}^{*}, \tilde{\mathbf{u}}^{i},\left\{\xi_{j}^{*}\right\}_{j=1, \ldots, m},\left\{\eta_{i}^{*}\right\}_{i=1, \ldots, n},\left\{\theta_{i}^{*}\right\}_{i=1, \ldots, n}\right)
$$

be the quantities defined in ROM.a. Now consider the following assignment of values 
to the dual variables

$$
\begin{aligned}
\beta_{i, \mathbf{v}_{-i}, \mathbf{u}_{i}, \mathbf{v}_{i}} & =0, \quad \forall \mathbf{v} \neq \mathbf{z}, \forall \mathbf{u}_{i}, \\
\beta_{i, \mathbf{v}_{-i}, \mathbf{v}_{i}, \mathbf{u}_{i}}=0, & \forall \mathbf{v} \neq \mathbf{z}, \forall \mathbf{u}_{i}, \\
\beta_{i, \mathbf{z}_{-i}, \mathbf{u}_{i}, \mathbf{z}_{i}}=0, & \forall \mathbf{v} \neq \mathbf{z}, \forall \mathbf{u}_{i}, \\
\sum_{\mathbf{u}_{i}} \beta_{i, \mathbf{z}_{-i}, \mathbf{u}_{i}, \mathbf{z}_{i}}^{i, \mathbf{z}_{i}}=1, & \\
\theta_{i,\left(\tilde{\mathbf{u}}^{i}, \mathbf{z}_{-i}\right)}=\theta_{i}^{*}, & \forall i \in \mathcal{N} .
\end{aligned}
$$

$$
\begin{aligned}
& \omega_{\mathbf{v}}= \begin{cases}1, & \text { if } \mathbf{v}=\mathbf{z}, \\
0, & \text { otherwise. }\end{cases} \\
& \eta_{i, \mathbf{v}}=\left\{\begin{array}{ll}
\eta_{i}^{*}, & \text { if } \mathbf{v}=\mathbf{z}, \\
0, & \text { otherwise, }
\end{array} \quad \forall i \in \mathcal{N} .\right. \\
& \xi_{j, \mathbf{v}}=\left\{\begin{array}{ll}
\xi_{j}^{*}, & \text { if } \mathbf{v}=\mathbf{z}, \\
0, & \text { otherwise, }
\end{array} \quad \forall j \in \mathcal{M} .\right.
\end{aligned}
$$

It is easy to verify that this is a dual feasible solution.

The primal feasible solution is calculated by using ROM.b and the feasibility follows from Propositions 15-17. The equality of the objective function values follows from Proposition 18. By strong duality, this conlcudes the proof for Part (a).

(b) Consider next a general bounded uncertainty set $\mathcal{U}$. Let $\mathrm{z}$ be as defined in (3.8). Let $\mathcal{U}^{\mathrm{D}}$ be a discrete subset of $\mathcal{U}$ that contains $\mathbf{z}$. Let $\mathcal{Q}$ represent the set of constraints (3.3)-(3.6). Since $\mathcal{U}^{\mathrm{D}} \subset \mathcal{U}$, we have the following

$$
\max _{\left\{\mathbf{x}^{\mathbf{v}}, \mathbf{p}^{\mathbf{v}}\right\} \in \mathcal{Q}} \min _{\mathbf{v} \in \mathcal{U}} W \leq \max _{\left\{\mathbf{x}^{\mathbf{v}}, \mathbf{p}^{\mathbf{v}}\right\} \in \mathcal{Q}} \min _{\mathbf{v} \in \mathcal{U}^{\mathbb{D}}} W
$$


By applying Part (a) for $\mathcal{U}^{\mathrm{D}}$, we have

$$
\max _{\left\{\mathbf{x}^{\mathbf{v}}, \mathbf{p}^{\mathbf{v}}\right\} \in \mathcal{Q}} \min _{\mathbf{v} \in \mathcal{U}^{\mathrm{D}}} W=\sum p_{i}^{\mathbf{z}}=Z^{*}\left(\mathcal{U}^{\mathrm{D}}\right)
$$

Since $\mathrm{z} \in \mathcal{U}$, and by using the allocation and payment rules of $R O M . b$, we have

$$
\max _{\left\{\mathbf{x}^{\mathbf{v}}, \mathbf{p}^{\mathbf{v}}\right\} \in \mathcal{Q}} \min _{\mathbf{v} \in \mathcal{U}} W \geq \sum p_{i}^{\mathbf{z}}=Z^{*}\left(\mathcal{U}^{\mathrm{D}}\right)
$$

Finally from Eqns. (3.49), (3.50) and (3.51), we have

$$
\max _{\left\{\mathbf{x}^{\mathbf{v}}, \mathbf{p}^{\mathbf{v}}\right\} \in \mathcal{Q} \mathbf{v} \in \mathcal{U}} \min _{\mathbf{U}} W=Z^{*}\left(\mathcal{U}^{\mathrm{D}}\right)
$$

which shows that the worst case revenue is optimal to (3.1). By using the combination of Propositions 15-17 shown for a general bounded uncertainty set $\mathcal{U}$, the result follows.

\subsubsection{Solving $R O M$}

The computationally intensive step in $R O M$ involves solving the bilinear optimization problems (3.8). Bilinear problems are NP-Hard (Sherali and Alameddine [1992]) for general uncertainty sets $\mathcal{U}$. However, if the uncertainty set $\mathcal{U}$ has a polynomial number of extreme points, then we can obtain a polynomial time algorithm that solves (3.8). This follows from Proposition 20 below, where we show that there exists an extreme point solution to these problems. Thus, we can solve the problems (3.8) in polynomial time, by simply enumerating all the extreme points.

Proposition 20. There exists an extreme point of $\mathcal{U}$ that is optimal to Problems (3.8).

Proof. The proof follows directly from the observation that both the inner and outer optimization problems are linear optimization problems. Thus, fixing the inner optimal solution, there always exists an extreme point solution to the outer problem and vice-versa. 
We next describe an algorithm to solve the bilinear optimization problem (3.8). This algorithm, motivated from the Generalized Benders Decomposition algorithm presented in Beran et al. [1997], is presented in Figure 3-3.

In Section 3.5, we present empirical evidence showing that for large scale problems and general uncertainty sets, $R O M$ is numerically tractable.

\subsubsection{The Case of Indivisible Items}

Until now we considered the case of the auction design problem in which the items can be divided among the buyers. When the items cannot be divided, then we are restricted to look for integral allocations of the items to buyers. We next provide a partial solution to the auction design problem with indivisible items, partial as the constraints are only satisfied in expectation, by considering a randomized version of ROM. This mechanism that we call ROM-Ind is presented in Figure 3-4.

Theorem 21. ROM-Ind

(a) is incentive-compatible, budget feasible, and individually rational, all in expectation;

(b) leads to a revenue of at least $Z^{*}(\mathcal{U})$, in expectation.

Proof. (a) The expected payment $\mathbb{E}\left[p_{i}(\mathrm{v})\right]$ charged to a buyer $i$ under $R O M$-Ind, is given by

$$
\begin{aligned}
\mathbb{E}\left[p_{i}(\mathbf{v})\right] & =\sum_{j \in \mathcal{M}}\left(a_{i j}^{\mathbf{v}} \cdot \frac{p_{i}^{\mathbf{v}}}{\sum_{j \in \mathcal{M}} a_{i j}^{\mathbf{v}}}\right) \\
& =p_{i}^{\mathbf{v}} \leq B_{i},
\end{aligned}
$$

which follows from the fact that $p_{i}^{\vee}$ is the payment computed by ROM.b which is a budget feasible mechanism. Thus, ROM-Ind is budget feasible in expectation. 


\section{Algorithm Generalized Benders Decomposition}

Input : Problem (3.8), accuracy parameter $\epsilon$. Output : Approximate optimal solution $\mathbf{z}$.

1. Set parameters $U B=\infty, L B=0, k=0$.

2. Compute $\mathbf{v}^{0}$ as follows:

- Compute $v_{1}^{0}=\min _{\mathbf{v} \in \mathcal{U}} v_{1}$.

- For each $i=2, \ldots,|\mathcal{N}|$

$$
v_{i}^{0}=\min _{\left(v_{1}^{0}, \ldots, v_{i-1}^{0}, v_{i}, \ldots, v_{n}\right) \in \mathcal{U}} v_{i} .
$$

3. While $U B-L B \geq \epsilon$,

- Solve the inner linear optimization problem (ILP) using $\mathbf{v}=\mathbf{v}^{k}$.

- Set $\mathbf{x}^{k}$ to be an optimal solution of ILP and update the value of $U B$ to the value of the optimal solution.

- Solve the outer linear optimization problem (OLP) using $\mathbf{x}=\mathbf{x}^{k}$.

- Set $\mathbf{v}^{k+1}$ to be an optimal solution of OLP, and update the value of $L B$ to the value of the optimal solution.

- Increment $k$.

- Add the constraint $\sum_{i \in \mathcal{N}} p_{i} \leq U B$ to ILP.

- Add the constraint $\sum_{i \in \mathcal{N}} \sum_{j \in \mathcal{M}} x_{i j} v_{i j} \geq L B$ to OLP.

4. Output $\mathbf{v}^{k}$.

Figure 3-3: Generalized Benders Decomposition Algorithm for Problem (3.8). 
Algorithm ROM-Ind

Input : Uncertainty set $\mathcal{U}$, budgets $B_{1}, \ldots, B_{n}$, and the bid vector $\mathrm{v}$.

Output : Allocation probabilities $\left\{a_{i j}^{\mathbf{v}}\right\}_{i \in \mathcal{N}, j \in \mathcal{M}}$ and payments .

1. Using ROM, compute the allocation vector and the prices $\left\{\left\{a_{i j}^{\mathbf{v}}\right\}_{i \in \mathcal{N}, j \in \mathcal{M}},\left\{p_{i}^{\mathbf{v}}\right\}_{i \in \mathcal{N}}\right\}$.

2. Allocate the $j^{\text {th }}$ item to $i^{\text {th }}$ buyer with probability $a_{i j}^{\mathbf{v}}$ and charge $\frac{p_{i}^{\mathbf{v}}}{\sum_{j \in \mathcal{M}} a_{i j}^{\mathbf{v}}}$ to the $i^{\text {th }}$ buyer.

Figure 3-4: Mechanism for Indivisible Items.

The expected utility derived by buyer $i$ is given by

$$
\begin{aligned}
\mathbb{E}\left[U_{i}(\mathbf{v})\right] & =\sum_{j \in \mathcal{M}} \mathbb{E}\left[\mathbf{1}_{\{\text {item } j \text { assigned to buyer } i\}} \cdot v_{i j}\right]-\mathbb{E}\left[p_{i}(\mathbf{v})\right] \\
& =\sum_{j \in \mathcal{M}} \mathbb{E}\left[\mathbf{1}_{\{\text {item } j \text { assigned to buyer } i\}}\right] \cdot v_{i j}-p_{i}^{\mathbf{v}} \\
& =\sum_{j \in \mathcal{M}} a_{i j}^{\mathbf{v}} \cdot v_{i j}-p_{i}^{\mathbf{v}}
\end{aligned}
$$

which is the same as the utility under ROM.b. Therefore, ROM-Ind inherits the properties of incentive compatibility and individual rationality from $R O M$, both in expectation. This concludes the proof.

(b) The expected revenue is given by $\sum_{i \in \mathcal{N}} p_{i}^{\vee}$ which is at least $Z^{*}(\mathcal{U})$ by Theorem 19.

\subsection{Single Item Auctions}

In this section, we consider a special case of the auction design problem, in which the buyers do not have any budget constraints. In the absence of budget constraints, the 
auction design problem for multiple items reduces to the auction design problem for a single item. Consequently we consider the auction design problem for a single item without budget constraints. Myerson [1981] solved this problem in a probabilistic setting for buyers with uncorrelated valuations and showed the optimal mechanism takes the form of a second price auction with a reservation price. We recover Myerson's result in a more general setting that allows correlated buyers and obtain an optimal mechanism that also takes the form of a second price auction with a reservation price.

\subsubsection{The Robust Optimal Mechanism for Single Item Auc- tions without Budget Constraints}

By specializing $R O M$ to the case $B_{i}=\infty, \forall i \in \mathcal{N}$ and $|\mathcal{M}|=1$, we derive the optimal mechanism for single item auctions without budget constraints, that we will refer to as ROM-Si. ROM-Si consists of Algorithms ROM-Si. $a$ and ROM-Si.b presented in Figures 3-5 and 3-6, respectively. Algorithm ROM-Si.a is obtained from ROM.a, by setting the budgets to $\infty$ and taking $|\mathcal{M}|=1$.

\section{Properties and Optimality of $R O M-S i$}

$R O M-S i$, as a speacial case of $R O M$, is worst case optimal, individually rational and incentive compatible. We next show that the allocation (3.57) and payment rules (3.58) reduce to the "second price mechanism with a reservation price $r^{*}$ ", see Vickrey [1961], Clarke [1971], Groves [1977].

We first present a simplification of ROM-Si.a to calculate the reservation price $r^{*}$ using a single optimization problem instead of using Eqns. (3.52) and (3.53). Note that the inner optimization problem of (3.52) given by

$$
\begin{aligned}
\max _{\left\{x_{i}\right\}_{i \in \mathcal{N}}} & \sum_{i \in \mathcal{N}} x_{i} \cdot v_{i} \\
\text { s.t. } & \sum_{i \in \mathcal{N}} x_{i} \leq 1, \\
& x_{i} \geq 0, \quad \forall i \in \mathcal{N}
\end{aligned}
$$


Algorithm ROM-Si.a

Input : Uncertainty set $\mathcal{U}$.

Output : Reservation price $r^{*}$.

1. Compute the worst case valuation vector $\mathbf{z}=\left\{z_{i}\right\}_{i \in \mathcal{N}}$ given by

$$
\mathbf{z}=\arg \min _{\mathbf{v} \in \mathcal{U}}\left\{\begin{array}{cc}
\max _{\left\{x_{i}\right\}_{i \in \mathcal{N}}} & \sum_{i \in \mathcal{N}} x_{i} \cdot v_{i} \\
\text { s.t. } & \sum_{i \in \mathcal{N}} x_{i} \leq 1 \\
& x_{i} \geq 0, \quad \forall i \in \mathcal{N}
\end{array}\right\}
$$

2. Compute reservation price $r^{*}$ given by

$$
r^{*}=\arg \left\{\begin{array}{cc}
\min _{r} & r \\
\text { s.t. } & r \geq z_{i}, \forall i \in \mathcal{N}
\end{array}\right\}
$$

Figure 3-5: Calculation of the reservation price.

has a simple optimal solution given by

$$
x_{k}^{*}=1, \Longleftrightarrow v_{k}=\max _{i} v_{i}
$$

Therefore, by combining this observation with Eqns. (3.52) and (3.53), we obtain that the reservation price $r^{*}$ is given by the following single optimization problem

$$
\begin{array}{cc}
\min _{r, \mathbf{v}} & r \\
\text { s.t. } & r \geq v_{i}, \forall i \in \mathcal{N}, \\
& \left(v_{1}, v_{2}, \ldots, v_{n}\right) \in \mathcal{U} .
\end{array}
$$

We next present an alternate equivalent version of ROM-Si.b, which has the form of a second price auction as presented in Figure 3-7. We show the equivalence in Proposition 22. 


\section{Algorithm ROM-Si.b}

Input : Bid vector $\mathrm{v}=\left\{v_{i}\right\}_{i \in \mathcal{N}}, r^{*}$.

Output : Allocation vector $\left\{a_{k}^{\mathrm{v}}\right\}_{k \in \mathcal{N}}$ and the payments $\left\{p_{k}^{\mathrm{v}}\right\}_{k \in \mathcal{N}}$.

1. Calculate the quantities $\left(\left\{y_{i}^{\mathbf{v}}, r_{i}^{\mathbf{v}}\right\}_{i \in \mathcal{N}},\left\{y_{i, k}^{\mathbf{v}-k}, r_{i, k}^{\mathbf{v}-k}\right\}_{i \in \mathcal{N} \backslash\{k\}}\right)$ given by

$$
\begin{aligned}
\left\{y_{i}^{\mathbf{v}}, r_{i}^{\mathbf{v}}\right\}_{i \in \mathcal{N}} & =\arg \max _{(\mathbf{y}, \mathbf{r}) \in \mathcal{P}} \sum_{i \in \mathcal{N}} y_{i} \cdot v_{i}-r_{i}, \\
\left\{y_{i, k}^{\mathbf{v}-k}, r_{i, k}^{\mathbf{v}-k}\right\}_{i \in \mathcal{N} \backslash\{k\}} & =\arg \max _{(\mathbf{y}, \mathbf{r}) \in \mathcal{P}} \sum_{i \in \mathcal{N} \backslash\{k\}} y_{i} \cdot v_{i}-r_{i},
\end{aligned}
$$

where

$$
\mathcal{P}=\left\{\begin{array}{l|l}
\left\{x_{i}, r_{i}\right\}_{i \in \mathcal{N}} & \begin{array}{l}
\sum_{i \in \mathcal{N}} x_{i} \leq 1, \\
\sum_{i \in \mathcal{N}} r_{i} \geq r^{*}, \\
x_{i} \geq 0, \quad \forall i \in \mathcal{N}
\end{array}
\end{array}\right\}
$$

2. Compute the allocation vector $\left\{a_{k}^{v}\right\}_{k \in \mathcal{N}}$ and the payments $\left\{p_{k}^{\mathbf{v}}\right\}_{k \in \mathcal{N}}$ as follows

$$
\begin{aligned}
& a_{k}^{\mathbf{v}}=y_{k}^{\mathbf{v}} \\
& p_{k}^{\mathbf{v}}=r_{k}^{\mathbf{v}}+\sum_{i \in \mathcal{N} \backslash\{k\}}\left(y_{i, k}^{\mathbf{v}-k} v_{i}-r_{i, k}^{\mathbf{v}-k}\right)-\sum_{i \in \mathcal{N} \backslash\{k\}}\left(y_{i}^{\mathbf{v}} v_{i}-r_{i}^{\mathbf{v}}\right) .
\end{aligned}
$$

Figure 3-6: Calculation of allocations and payments. 
Algorithm Second Price Auction with a reservation price

Input : Bid vector $\mathbf{v}=\left\{v_{i}\right\}_{i \in \mathcal{N}}, r^{*}$.

Output : Allocation vector $\left\{a_{k}^{\mathbf{v}}\right\}_{k \in \mathcal{N}}$ and the payments $\left\{p_{k}^{\mathbf{v}}\right\}_{k \in \mathcal{N}}$.

1. Reject each buyer $i$ with $v_{i}<r^{*}$, and let $\mathcal{N}^{\prime}=\left\{i \in \mathcal{N} \mid v_{i} \geq r^{*}\right\}$.

2. Allocate the item to the buyer $h \in \mathcal{N}^{\prime}$ with the highest valuation.

3. Charge buyer $h$ a price given by

$$
p_{h}=\max \left(r^{*}, \max _{i \in \mathcal{N}^{\prime} \backslash\{h\}} v_{i}\right)
$$

which corresponds to the second highest valuation greater than $r^{*}$.

Figure 3-7: The Second Price Auction with a reservation price.

Proposition 22. ROM-Si.b is equivalent to a second price auction with a reservation price $r^{*}$.

Proof. In ROM-Si.b, the allocations are computed by solving the linear optimization problem (3.54). Let

$$
\left\{y_{i}^{\mathbf{v}}, r_{i}^{\mathbf{v}}\right\}_{i \in \mathcal{N}}=\arg \left\{\begin{array}{cc}
\max _{\left\{y_{i}, r_{i}\right\}_{i \in \mathcal{N}}} & \sum_{i \in \mathcal{N}}\left(y_{i} \cdot v_{i}-r_{i}\right) \\
\text { s.t. } & \sum_{i \in \mathcal{N}} y_{i} \leq 1 \\
& \sum_{i \in \mathcal{N}} r_{i} \geq r^{*} \\
& x_{i} \geq 0, \forall i \in \mathcal{N}
\end{array}\right\}
$$

and therefore, at optimality we have

$$
\sum_{i \in \mathcal{N}} r_{i}^{\mathbf{v}}=r^{*}
$$


Therefore, (3.60) is equivalent to

$$
\left\{y_{i}^{\mathbf{v}}, r_{i}^{\mathbf{v}}\right\}_{i \in \mathcal{N}}=\arg \left\{\begin{array}{cc}
\max _{\left\{y_{i}, r_{i}\right\}_{i \in \mathcal{N}}} & \sum_{i \in \mathcal{N}} y_{i} \cdot v_{i} \\
\text { s.t. } & \sum_{i \in \mathcal{N}} y_{i} \leq 1 \\
& \sum_{i \in \mathcal{N}} r_{i}=r^{*} \\
& x_{i} \geq 0, \quad \forall i \in \mathcal{N}
\end{array}\right\} \text {. }
$$

Consider the following feasible solution

$$
\begin{aligned}
& y_{i}^{\mathbf{v}}= \begin{cases}0, & \text { if } i \neq h, \\
1, & \text { if } i=h,\end{cases} \\
& r_{i}^{\mathbf{v}}= \begin{cases}0, & \text { if } i \neq h, \\
r_{i}^{*}, & \text { if } i=h,\end{cases}
\end{aligned}
$$

where $h$ is equal to one of the solutions of

$$
\arg \max _{i \in \mathcal{N}} v_{i}
$$

Clearly, this solution is optimal to (3.61) and therefore to (3.60). Therefore, from (3.62), the allocation steps are equivalent.

We next consider the payment rule in ROM-Si.b, given by (3.58). If $k \neq h$, we know from (3.62) and (3.63) that

$$
\sum_{i \in \mathcal{M} \backslash k\}} y_{i}^{\mathbf{v}} \cdot v_{i}-r_{i}=y_{h}^{\mathbf{v}} \cdot v_{h}-r_{h}=v_{h}-r^{*}
$$

We next consider the expression $\sum_{i \in \mathcal{N} \backslash\{k\}} y_{i, k}^{\mathbf{v}_{-k}} \cdot v_{i}-r_{i, k}^{\mathbf{v}_{-k}}$, where $\left\{y_{i, k}^{\mathbf{v}_{-k}}\right\}$ are defined as the solutions of the linear optimization problem (3.55). By the same argument that 
led us to (3.62), we have

$$
y_{i, k}^{\mathbf{v}_{-k}}= \begin{cases}0, & \text { if } i \neq h \\ 1, & \text { if } i=h\end{cases}
$$

which implies that

$$
\sum_{i \in \mathcal{M} \backslash\{k\}} y_{i, k}^{\mathbf{v}-k} \cdot v_{i}-r_{i, k}^{\mathbf{v}-k}=y_{h, k}^{\mathbf{v}-k} \cdot v_{h}-r_{h, k}^{\mathbf{v}-k}=v_{h}-r^{*}
$$

Therefore, from (3.64) and (3.65) we have $p_{k}=0$, if $k \neq h$.

When $k=h$, we have

$$
\begin{aligned}
p_{h} & =r_{h}^{\mathbf{v}}+\sum_{i \in \mathcal{N} \backslash\{h\}}\left(y_{i, h}^{\mathbf{v}-h} \cdot v_{i}-r_{i, h}^{\mathbf{v}-h}\right)-\sum_{i \in \mathcal{N} \backslash\{h\}}\left(y_{i}^{\mathbf{v}} \cdot v_{i}-r_{i}^{\mathbf{v}}\right) \\
& =r^{*}+\sum_{i \in \mathcal{N} \backslash\{h\}}\left(y_{i, h}^{\mathbf{v}-h} \cdot v_{i}-r_{i, h}^{\mathbf{v}-h}\right) .
\end{aligned}
$$

We next consider the value of $\sum_{i \in \mathcal{N} \backslash\{h\}}\left(y_{i, h}^{\mathbf{v}-h} \cdot v_{i}-r_{i, h}^{\mathbf{v}-h}\right)$. We know that, using a similar argument leading to (3.62) and (3.63),

$$
\sum_{i \in \mathcal{N} \backslash\{h\}}\left(y_{i, h}^{\mathbf{v}_{-h}} \cdot v_{i}-r_{i, h}^{\mathbf{v}_{-h}}\right)=v_{h^{\prime}}-r^{*}
$$

where

$$
h^{\prime}=\arg \max _{i \in \mathcal{N} \backslash\{h\}} v_{i},
$$

is the buyer with the second highest valuation (after buyer $h$ ). Therefore, the payment charged to buyer $h$ is given by

$$
p_{h}=v_{h^{\prime}}
$$

This concludes the proof. 


\subsubsection{Closed Form Solutions}

In this section, we obtain closed form solutions for the worst case optimal revenues obtained under ROM for (a) single item auctions with and without budgets and (b) multiple items with equal budgets.

Proposition 23. The worst case optimal revenue $R^{*}$, for single item auction with $n$ buyers who do not have budget constraints are as follows:

(a) For uncertainty set

$$
\left.\begin{array}{c}
\mathcal{U}^{C L T}=\left\{\left(v_{1}, \ldots, v_{n}\right) \mid-\Gamma \leq \frac{\sum_{i=1}^{n} v_{i}-n \cdot \mu}{\sqrt{n} \cdot \sigma} \leq \Gamma\right.
\end{array}\right\},
$$

(b) For uncertainty set

$$
\mathcal{U}^{C o r r}=\left\{\left(v_{1}, \ldots, v_{n}\right) \mid \begin{array}{c}
v_{i}=f+n_{i}, \quad \forall i \in \mathcal{N}, \\
\underline{F} \leq f \leq \bar{F} \\
-\Gamma \leq \frac{\sum_{i=1}^{n} n_{i}-n \cdot \nu}{\sqrt{n} \cdot \gamma} \leq \Gamma \\
R^{*}=\underline{F}+\nu-\frac{\Gamma \cdot \gamma}{\sqrt{n}}
\end{array}\right\}
$$

(c) For uncertainty set

$$
\mathcal{U}^{\text {Normal }}=\left\{\left(v_{1}, \ldots, v_{n}\right) \mid n \sigma^{2}-\Gamma \leq \sum_{i=1}^{n}\left(v_{i}-\mu\right)^{2} \leq n \sigma^{2}+\Gamma\right\}
$$




$$
\begin{aligned}
R^{*} & =\mu+\sqrt{\sigma^{2}-\frac{\Gamma}{n}} \\
& \approx \mu+\sigma, \text { for large } n .
\end{aligned}
$$

(d) For uncertainty set

$$
\begin{gathered}
\mathcal{U}^{f-\text { Typical }}=\left\{\left(v_{1}, \ldots, v_{n}\right) \mid-\Gamma \leq \frac{\sum_{i=1}^{n} \log f\left(v_{i}\right)-n \cdot \mu_{\log f}}{\sigma_{\log f} \sqrt{n}} \leq \Gamma\right\} \\
R^{*}=\exp \left(\mu_{\log f}-\frac{\Gamma \cdot \sigma_{\log f}}{\sqrt{n}}\right) .
\end{gathered}
$$

Proof. (a) The proof follows from the fact that there exists an optimal solution $\mathbf{v}^{*}$ to (3.59) such that $v_{i}^{*}=v_{j}^{*} \forall i, j$. For the purpose of deriving a contradiction, suppose $\mathbf{v}$ is an optimal solution such that the components of $\mathbf{v}$ are all not equal. Consider another valuation vector $\mathbf{v}^{*}$ such that

$$
v_{k}^{*}=\frac{\sum_{i=1}^{n} v_{i}}{n}, \forall k=1, \ldots, n
$$

Since $\sum_{i=1}^{n} v_{i}^{*}=\sum_{i=1}^{n} v_{i}$, the new vector $\mathrm{v}^{*}$ is feasible in $\mathcal{U}^{\mathrm{CLT}}$. Also the objective value in (3.59) can only decrease under this new valuation vector. Therefore, $\mathbf{v}^{*}$ is also an optimal solution. We also have

$$
\sum_{i=1}^{n} v_{i}^{*} \geq n \mu-\Gamma \sigma \sqrt{n}
$$

which has to be an equality at optimality. This concludes the proof.

(b), (c), (d) The proof is similar to (a) and is thus omitted.

We note that under uncertainty set $\mathcal{U}^{\mathrm{f} \text {-Typical }}$ the reservation price is equal to $\exp (H)$, where $-H$ is the entropy of the valuation distribution.

Proposition 24. (a) The worst case revenue $R^{*}$ for a single item auction with $n$ 
buyers with budget constraints $\left\{B_{i}\right\}_{i \in \mathcal{N}}$, and when the auctioneer assumes that the valuations come from the uncertainty set $\mathcal{U}^{C L T}$ is given by

$$
R^{*}=\min \left\{\sum_{i \in \mathcal{N}} B_{i}, \mu-\frac{\Gamma \sigma}{\sqrt{n}}\right\}
$$

(b) The worst case revenue $R^{*}$ for an $m$-item auction with $n$ buyers with equal budget constraints $B$, and when the auctioneer assumes that the valuations come from the uncertainty set $\mathcal{U}^{C L T}$ is given by

$$
R^{*}=\min \left\{n B, m\left(\mu-\frac{\Gamma \sigma}{\sqrt{n}}\right)\right\}
$$

Proof. The proof is similar to the proof of Proposition 23(a), and is thus omitted.

\subsubsection{Comparison with the Myerson Auction}

As we showed in Proposition 22, ROM-Si and the Myerson auction have the same structure, that of a second price auction with a reservation price. However, the mechanisms differ in the way they calculate the reservation prices. In the case of the Myerson auction the reservation price is calculated by solving a non-linear equation

$$
\frac{1-F(r)}{f(r)}=r
$$

where $F(\cdot)$ is the cdf and $f(\cdot)$ is the pdf of the probability distribution that the auctioneer assumes the valuations are sampled from. On the other hand, in ROM-Si, the reservation price is calculated using the linear optimization problem given by Eq. (3.59).

In this section, we compare $R O M-S i$ and the Myerson auction with respect to the following three aspects: (a) Computational complexity, (b) Robustness properties, and (c) Sensitivity to the uncertainty set. 


\section{Computational Complexity}

The computationally intensive step in both $R O M-S i$ and the Myerson auction is the calculation of the reservation price. Once the reservation price is calculated, both these mechanisms solve linear optimization problems to carry out the auction. While the Myerson auction solves the non-linear equation (3.66), ROM-Si solves the optimization problem (3.59) to calculate the reservation price. As long as the uncertainty set $\mathcal{U}$ is convex, this optimization problem is polynomial time solvable. In particular, when $\mathcal{U}$ is a polyhedron, the optimization problem reduces to a linear optimization problem.

\section{Robustness Properties}

The values of the reservation prices obtained by ROM-Si and by the Myerson auction differ in general. For example, when we use the uncertainty set $\mathcal{U}^{\mathrm{CLT}}$ with parameters $\mu$ and $\sigma$, Proposition 23(a) gives $r^{*}=\mu-\frac{\Gamma \cdot \sigma}{\sqrt{n}} \approx \mu$ (for large $\mathrm{n}$ ).

On the other hand, the Myerson auction gives different values of reservation prices for different distributions. For uniform and exponential distributions, the reservation prices obtained by the auctions match, while for other distributions, the reservation prices are different. This dependence of reservation prices on the distribution may lead to lack of robustness on the part of the Myerson auction, when the assumed distribution differs from the realized distribution.

In order to study this, we design the following experiments. We first assume that valuations are normally distributed with parameters $\mu=1$, and $\sigma=0.5,1,2$ and carry out $\mathrm{ROM}-\mathrm{Si}$ and the Myerson auctions with these parameters. Then, we investigate how these auctions compare with each other, when the realized distributions are different from the assumed distributions. This is done by computing the quantity Relative Revenue defined as

$$
\text { Relative Revenue }=\frac{\text { ROM-Single Revenue }- \text { Myerson Revenue }}{\text { Myerson Revenue }}
$$

which when positive, indicates that the proposed auction results in a greater revenue 


\begin{tabular}{|c|c|c|c|}
\hline Distribution $/(\mu, \sigma)$ & $(1,0.5)$ & $(1,1)$ & $(1,2)$ \\
\hline \hline Gamma & 0.529 & 0.696 & 1.038 \\
\hline Beta & 0.387 & 0.507 & 0.799 \\
\hline Triangle & 0.271 & 0.376 & 0.526 \\
\hline Uniform & 0.498 & 0.697 & 0.959 \\
\hline
\end{tabular}

Table 3.1: Myerson vs ROM-Si: The Relative Revenue, defined in (3.67) for different distributions with the same mean and standard deviation.

\begin{tabular}{|c|c|c|c|}
\hline Standard Deviation $/(\mu, \sigma)$ & $(1,0.5)$ & $(1,1)$ & $(1,2)$ \\
\hline \hline$\sigma / 4$ & 0.108 & 0.134 & 0.196 \\
\hline$\sigma / 2$ & 0.0357 & 0.042 & 0.068 \\
\hline $3 \sigma / 2$ & 0.0282 & 0.039 & 0.062 \\
\hline $2 \sigma$ & 0.141 & 0.187 & 0.261 \\
\hline $5 \sigma$ & 0.247 & 0.334 & 0.542 \\
\hline
\end{tabular}

Table 3.2: Myerson vs ROM-Si: The Relative Revenue under the same mean but different standard deviation.

than the Myerson auction.

In Table 3.1, we compare the expected revenues (obtained by simulation) of the ROM-Si and of the Myerson auction, when the realized distribution is Gamma, Beta, Uniform and Triangle with the same mean and standard deviation. We find that the proposed approach has very significant benefits with revenue improvements in the range of $[27 \%, 103 \%]$. To amplify this further, we perform another experiment where we vary the distributions at a slower pace. In particular, we consider a series of distributions $\mathcal{F}$ that are increasingly different from $N(1,0.5)$ with respect to the value of total variation distance. The total variation distance between probability measures $\mathcal{F}_{1}$ and $\mathcal{F}_{2}$ is defined as the largest possible difference between the probabilities that

\begin{tabular}{|c|c|c|c|}
\hline Mean $/(\mu, \sigma)$ & $(1,0.5)$ & $(1,1)$ & $(1,2)$ \\
\hline \hline$\mu / 4$ & 0.178 & 0.22 & 0.335 \\
\hline$\mu / 4$ & 0.053 & 0.064 & 0.09 \\
\hline $2 \mu$ & -0.176 & -0.242 & -0.392 \\
\hline $3 \mu$ & -0.312 & -0.416 & -0.58 \\
\hline
\end{tabular}

Table 3.3: Myerson vs ROM-Si: The Relative Revenue under the same standard deviation but different mean. 


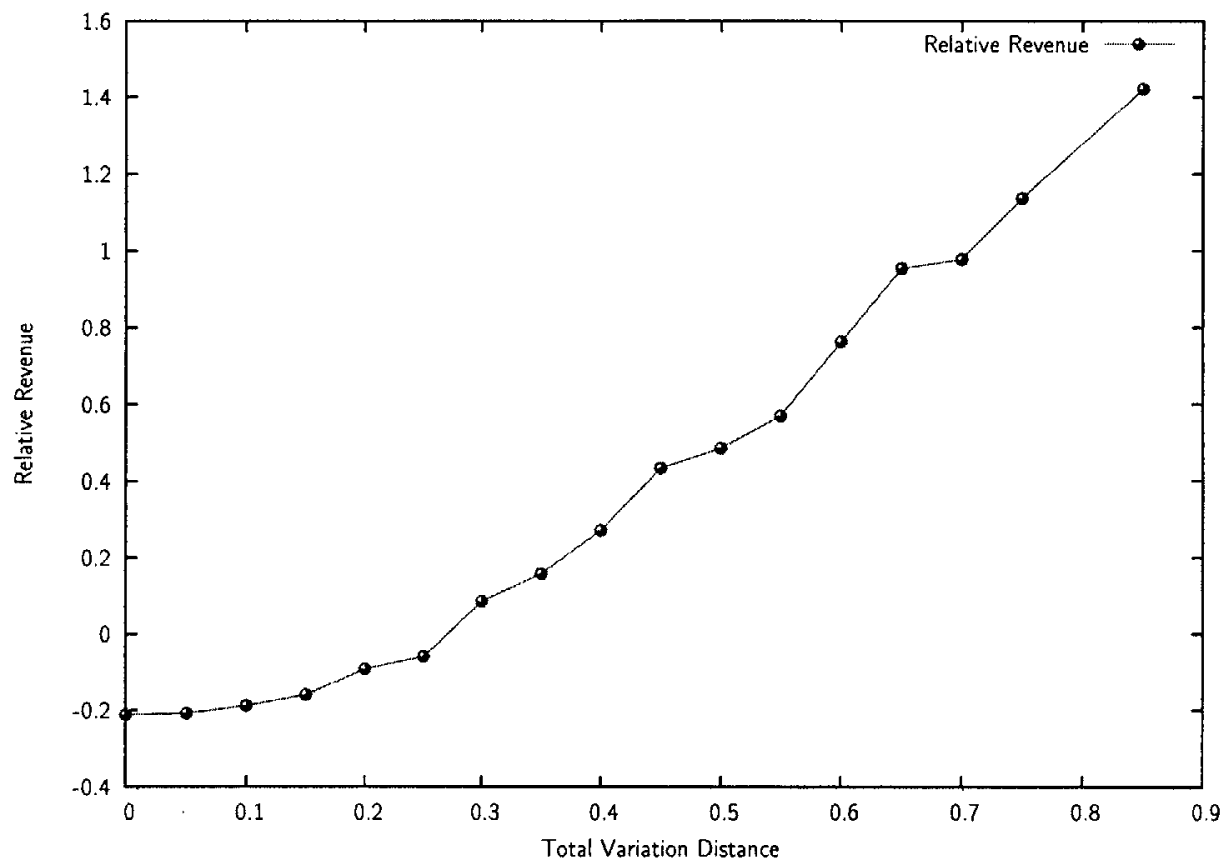

Figure 3-8: Robustness of ROM-Si.

$\mathcal{F}_{1}$ and $\mathcal{F}_{2}$ can assign to the same event, that is,

$$
\left\|\mathcal{F}_{1}-\mathcal{F}_{2}\right\|_{T V}=\sup _{A \in \Omega}\left|\mathcal{F}_{1}(A)-\mathcal{F}_{2}(A)\right|
$$

We plot the Relative Revenue against the values of total variation distances in Figure 8. We observe that ROM-Si performs better than the Myerson auction when the total variation distance becomes larger than 0.22 .

In Table 3.2, we compare the expected revenues of the ROM-Si and of the Myerson auction when the distribution is still normal with the same mean but with different standard deviations. We find that the proposed approach still has potentially significant benefits in the range of $[2.8 \%, 54 \%]$. In Table 3.3, we investigate the situation when the realized distribution is again normal with the same standard deviation but with different means. We find that the results in this case are mixed. When the realized mean is smaller (larger) than the assumed mean, the proposed approach outperforms (underperforms) when compared with the Myerson auction. In summary, 


\begin{tabular}{|c|c|c|c|c|}
\hline$\mu$ & $\sigma^{2}$ & $r^{*}$ under Myerson & $r^{*}$ under $\mathcal{U}^{\text {CLT }}$ & $r^{*}$ under $\mathcal{U}^{\text {Normal }}$ \\
\hline \hline 1 & 0.5000 & 1.4876 & 1 & 1.7071 \\
\hline 1 & 1.0000 & 1.7640 & 1 & 2.0000 \\
\hline 1 & 2.0000 & 2.3145 & 1 & 2.4142 \\
\hline 2 & 1.0000 & 2.7645 & 2 & 3.0000 \\
\hline 2 & 2.0000 & 3.1983 & 2 & 3.4142 \\
\hline 2 & 3.0000 & 3.5634 & 2 & 3.7321 \\
\hline 2 & 4.0000 & 3.7983 & 2 & 4.0000 \\
\hline 3 & 2.0000 & 4.2876 & 3 & 4.4142 \\
\hline 3 & 4.0000 & 4.7645 & 3 & 5.0000 \\
\hline
\end{tabular}

Table 3.4: Comparison of reservation prices when valuations obey $N(\mu, \sigma)$.

we feel that $R O M-S i$ has stronger robustness properties when the distribution or the standard deviation is misspecified.

\section{Sensitivity to the uncertainty set}

In this section, we compare the reservation prices we obtain when we assume that valuations belong to the uncertainty set $\mathcal{U}^{\mathrm{CLT}}$ and $\mathcal{U}^{\text {Normal }}$ with the reservation prices we obtain under the Myerson auction when the auctioneer believes that valuation of the item obeys a normal distribution with mean $\mu$ and standard deviation $\sigma$.

In Table 3.4, we present the reservation prices obtained under the Myerson auction, and under ROM-Si when we use the uncertainty sets $\mathcal{U}^{\mathrm{CLT}}$ and $\mathcal{U}^{\text {Normal }}$. Note the closeness of the reservation prices under the Myerson auction and under ROM-Si with $\mathcal{U}^{\text {Normal }}$. This is not unexpected given that the $\mathcal{U}^{\text {Normal }}$ was designed by using knowledge of the probability distribution.

\subsection{Multi-Item Auctions with Private Budgets}

Until now we have assumed that the budgets are all known to the auctioneer, which might not hold in many practical situations. In the absence of budget information, Dobzinski et al. [2008] shows that there is no deterministic auction that

(a) is individually rational and dominant-strategy incentive compatible, 
(b) makes no positive transfers, and

(c) always produces a Pareto-optimal outcome,

when one uses a probabilistic model to capture the uncertainty in the budgets. In this section, we propose an uncertainty set based model for handling the uncertainty in the budgets, that allows us to avoid this impossibility result. In particular, we assume that the auctioneer has access to an uncertainty set $\mathcal{B}$ representing the uncertainty in the budget vectors. Such an uncertainty set can be constructed using the methods discussed in Section 2.1. For example, if we have access to the mean $\mu^{b}$ and the standard deviation $\sigma^{b}$ of the budgets, then based on the central limit theorem, we can construct a budget uncertainty set $\mathcal{B}^{\mathrm{CLT}}$ given by

$$
\mathcal{B}^{\mathrm{CLT}}=\left\{\left(b_{1}, \ldots, b_{n}\right) \mid-\Gamma^{b} \leq \frac{\sum_{i=1}^{n} b_{i}-n \cdot \mu^{b}}{\sqrt{n} \cdot \sigma^{b}} \leq \Gamma^{b}\right\} .
$$

With such a model of uncertainty in budgets, the optimization problem that solves for the worst case optimal auction is given in (3.68). The decision variables $\mathbf{x}^{\mathbf{v}, \mathbf{b}}$ and $\mathrm{p}^{\mathbf{v}, \mathbf{b}}$ represent the allocations and the payments, respectively, for each valuation vector $\mathbf{v} \in \mathcal{U}$, and for each budget vector $\mathbf{b} \in \mathcal{B}$. That is, if the realized valuation vector is $\mathbf{v}$ and the realized budget vector is $\mathbf{b}$, then we allocate a fraction $x_{i j}^{\mathbf{v}, \mathbf{b}}$ of item $j$ to buyer $i$, and charge a total of $p_{i}^{\mathbf{v}, \mathbf{b}}$ to the $i^{\text {th }}$ buyer. 


$$
\begin{aligned}
Z_{b}^{*}=\max & W \\
\text { s.t. } \quad & W \leq \sum_{i=1}^{n} p_{i}^{\mathbf{v}, \mathbf{b}}, \forall \mathbf{v} \in \mathcal{U}, \forall \mathbf{b} \in \mathcal{B} \\
& \sum_{i=1}^{n} x_{i j}^{\mathbf{v}, \mathbf{b}} \leq 1, \quad \forall j \in \mathcal{M}, \forall \mathbf{v} \in \mathcal{U}, \forall \mathbf{b} \in \mathcal{B}, \\
& \sum_{j \in \mathcal{M}} v_{i j} \cdot x_{i j}^{\left(\mathbf{u}_{i}, \mathbf{v}_{-i}\right), \mathbf{b}}-p_{i}^{\left(\mathbf{u}_{i}, \mathbf{v}_{-i}\right), \mathbf{b}}-\sum_{j \in \mathcal{M}} v_{i j} \cdot x_{i j}^{\left(\mathbf{v}_{i}, \mathbf{v}_{-i}\right), \mathbf{b}} \\
& +p_{i}^{\left(\mathbf{v}_{i}, \mathbf{v}_{-i}\right), \mathbf{b}} \leq 0, \forall\left(\mathbf{v}_{i}, \mathbf{v}_{-i}\right) \in \mathcal{U}, \forall\left(\mathbf{u}_{i}, \mathbf{v}_{-i}\right) \in \mathcal{U}, \forall i \in \mathcal{N}, \\
& p_{i}^{\mathbf{v}, \mathbf{b}} \leq b_{i}, \quad \forall i \in \mathcal{N}, \mathbf{v} \in \mathcal{U}, \forall \mathbf{b} \in \mathcal{B}, \\
& \sum_{j \in \mathcal{M}} v_{i j} \cdot x_{i j}^{\mathbf{v}, \mathbf{b}}-p_{i}^{\mathbf{v}, \mathbf{b}} \geq 0, \quad \forall i \in \mathcal{N}, \mathbf{v} \in \mathcal{U}, \forall \mathbf{b} \in \mathcal{B}, \\
& \mathbf{x}^{\mathbf{v}, \mathbf{b}} \geq \mathbf{0} .
\end{aligned}
$$

\subsubsection{The Robust Optimal Mechanism for Auctions with Bud- get Uncertainty}

We next present a mechanism to compute an optimal solution to the optimization problem (3.68). This mechanism, which we refer to as ROM-UB, consists of Algorithms ROM-UB.a and ROM-UB.b, presented in Figures 3-9 and 3-10, respectively. This mechanism, being an extension of ROM gives rise to a similar structural result, that is, it is optimal to use reservation prices. Furthermore, the reservation prices can be computed from a bilinear optimization problem which is computationally difficult but feasible, in sharp contrast with the impossibility results in Dobzinski et al. [2008] under probabilistic assumptions on budgets.

Theorem 25. ROM-UB is an incentive-compatible, individually rational and budget feasible mechanism achieving the worst case optimal revenue of $Z_{b}^{*}$.

Proof. The proof is similar to that of Theorem 19, and is thus omitted.

\subsection{Computational Results}

In this section, we address 


\section{Algorithm ROM-UB.a}

Input : Valuations uncertainty $\operatorname{set} \mathcal{U}$, and budgets uncertainty set $\mathcal{B}$.

Output : Reservation prices $\left\{r_{i j}^{*}\right\}_{i \in \mathcal{N}, j \in \mathcal{M}}$, and nominal allocations $\left\{x_{i j}^{*}\right\}_{i \in \mathcal{N}, j \in \mathcal{M}}$.

1. Compute the worst case valuation vector $\mathbf{z}=\left\{z_{i j}\right\}_{i \in \mathcal{N}, j \in \mathcal{M}}$, the worst case budget vector $\mathbf{d}=\left\{d_{i}\right\}_{i \in \mathcal{N}}$ and the nominal allocation vector $\mathbf{x}^{*}=\left\{x_{i j}^{*}\right\}_{i \in \mathcal{N}, j \in \mathcal{M}}$ given by

$\left(\mathbf{z}, \mathbf{d}, \mathbf{x}^{*}\right)=\arg \max _{\mathbf{v} \in \mathcal{U}} \min _{\mathbf{b} \in \mathcal{B}}\left\{\begin{array}{cc}\max _{\left\{x_{i j}\right\}_{i \in \mathcal{N}, j \in \mathcal{M}}} & \sum_{i \in \mathcal{N}, j \in \mathcal{M}} \sum_{i j} x_{i j} v_{i j} \\ \text { s.t. } & \sum_{i \in \mathcal{N}} x_{i j} \leq 1, \forall j \in \mathcal{M} . \\ & \sum_{j \in \mathcal{M}} x_{i j} \cdot v_{i j} \leq b_{i}, \forall i \in \mathcal{N}, \\ & \sum_{j \in \mathcal{M}} x_{i j} \cdot v_{i j} \leq \sum_{j \in \mathcal{M}} x_{i j} \cdot u_{i j}, \forall \mathbf{u} \in \mathcal{U}, \forall i \in \mathcal{N}, \\ & \mathbf{x} \geq \mathbf{0} .\end{array}\right\}$

2. Compute $\left(\boldsymbol{\xi}^{*}=\left\{\xi_{j}^{*}\right\}_{j \in \mathcal{M}}, \eta^{*}=\left\{\eta_{i}^{*}\right\}_{i \in \mathcal{N}}, \theta^{*}=\left\{\theta_{i}^{*}\right\}_{i \in \mathcal{N}}\right)$ given by

$$
\left(\boldsymbol{\xi}^{*}, \boldsymbol{\eta}^{*}, \boldsymbol{\theta}^{*}\right)=\arg \left\{\begin{array}{cc}
\min _{\left\{\xi_{j}, \eta_{i}\right\}} & \sum_{j \in \mathcal{M}} \xi_{j}+\sum_{i \in \mathcal{N}}\left(\eta_{i} d_{i}+\theta_{i} \sum_{j \in \mathcal{M}} x_{i j}^{*} \tilde{u}_{j}^{i}\right) \\
\text { s.t. } & \xi_{j}+z_{i j} \cdot \eta_{i}+z_{i j} \cdot \theta_{i} \geq z_{i j}, \forall i \in \mathcal{N}, \forall j \in \mathcal{M}, \\
\xi_{j}, \eta_{i}, \theta_{i} \geq 0 .
\end{array}\right\},
$$

where

$$
\tilde{\mathbf{u}}^{i}=\arg \min _{\mathbf{u} \in \mathcal{U}} \sum_{j \in \mathcal{M}} x_{i j}^{*} \cdot u_{i j}, \forall i \in \mathcal{N}
$$

3. Compute the reservation prices $\left\{r_{i j}^{*}\right\}_{i \in \mathcal{N}, j \in \mathcal{M}}$ given by

$$
r_{i j}^{*}=\xi_{j}^{*}+\eta_{i}^{*} \cdot z_{i j}+\theta_{i}^{*} \cdot z_{i j}
$$

Figure 3-9: Calculation of the reservation prices and the nominal allocations. 


\section{Algorithm ROM-UB.b}

Input : Bid vector $\mathbf{v}=\left\{v_{i j}\right\}_{i \in \mathcal{N}, j \in \mathcal{M}},\left\{r_{i j}^{*}\right\}_{i \in \mathcal{N}, j \in \mathcal{M}},\left\{x_{i j}^{*}\right\}_{i \in \mathcal{N}, j \in \mathcal{M}}, \mathbf{z}$, and $\mathbf{d}$.

Output : Allocation vector $\left\{a_{i j}^{\mathrm{v}}\right\}_{i \in \mathcal{N}, j \in \mathcal{M}}$ and the payments $\left\{p_{k}^{\mathrm{v}}\right\}_{k \in \mathcal{N}}$.

1. Check if $\mathrm{v} \in \mathcal{U}$. If $\mathrm{v} \notin \mathcal{U}$ then do not allocate anything to anybody and charge zero.

2. Calculate the adapted allocations $y_{i j}^{\mathbf{v}}$ and temporary quantities $y_{i j, k}^{\mathbf{v}}$ given by

$$
\begin{aligned}
\left\{y_{i j}^{\mathbf{v}}\right\}_{i \in \mathcal{N}, j \in \mathcal{M}} & =\arg \max _{\mathbf{y} \in \mathcal{P}} \sum_{i \in \mathcal{N}} \sum_{j \in \mathcal{M}} y_{i j} \cdot\left(v_{i j}-r_{i j}^{*}\right) \\
\left\{y_{i j, k}^{\mathbf{v}-k}\right\}_{i \in \mathcal{M} \backslash\{k\}, j \in \mathcal{M}} & =\arg \max _{\mathbf{y} \in \mathcal{Q}_{k}} \sum_{i \in \mathcal{N} \backslash\{k\}} \sum_{j \in \mathcal{M}} y_{i j} \cdot\left(v_{i j}-r_{i j}^{*}\right),
\end{aligned}
$$

where the sets $\left(\mathcal{P}, \mathcal{Q}_{1}, \ldots, \mathcal{Q}_{n}\right)$ are given by

$$
\begin{aligned}
& \mathcal{P}=\left\{\begin{array}{c|cc}
\sum_{i \in \mathcal{N}} y_{i j} \leq 1-\sum_{+}^{n \times m} x_{i j}^{*}, & \forall j \in \mathcal{M}, \\
\sum_{j \in \mathcal{M}} y_{i j} \cdot u_{i j} \leq d_{i}-\sum_{j \in \mathcal{M}} x_{i j}^{*} \cdot r_{i j}^{*} & \\
+\sum_{j \in \mathcal{M}} x_{k j}^{*} \cdot \theta_{i}^{*} \cdot \tilde{u}_{j}^{i}, & \forall \mathbf{u} \in \mathcal{U}, \forall i \in \mathcal{N}
\end{array}\right\} \\
& \mathcal{Q}_{k}=\left\{\begin{array}{c|cc}
\mathbf{y} \in \mathbb{R}_{+}^{n \times m} & \sum_{i \in \mathcal{N} \backslash\{k\}} y_{i j} \leq 1-\sum_{i \in \mathcal{N}} x_{i j}^{*}, & \forall j \in \mathcal{M}, \\
\sum_{j \in \mathcal{M}} y_{i j} \cdot u_{i j} \leq d_{i}-\sum_{j \in \mathcal{M}} x_{i j}^{*} \cdot r_{i j}^{*}, & \forall \mathbf{u} \in \mathcal{U}, \forall i \in \mathcal{N} \backslash\{k\}
\end{array}\right\}
\end{aligned}
$$

3. Compute the allocation vector $\left\{a_{i j}^{\mathbf{v}}\right\}_{i \in \mathcal{N}, j \in \mathcal{M}}$ and the payments $\left\{p_{k}^{\mathbf{v}}\right\}_{k=1, \ldots, n}$ as follows

$$
\begin{aligned}
a_{i j}^{\mathbf{v}}= & y_{i j}^{\mathbf{v}}+x_{i j}^{*} \\
p_{k}^{\mathbf{v}}= & \sum_{j \in \mathcal{M}} y_{k j}^{\mathbf{v}} \cdot r_{k j}^{*}+\sum_{j \in \mathcal{M}} x_{k j}^{*} \cdot r_{k j}^{*}-\sum_{j \in \mathcal{M}} x_{k j}^{*} \cdot \theta_{k}^{*} \cdot \tilde{u}_{j}^{k} \\
& +\sum_{i \in \mathcal{N} \backslash\{k\}} \sum_{j \in \mathcal{M}} y_{i j, k}^{\mathbf{v}-k} \cdot\left(v_{i j}-r_{i j}^{*}\right)-\sum_{i \in \mathcal{N} \backslash\{k\}} \sum_{j \in \mathcal{M}} y_{i j}^{\mathbf{v}} \cdot\left(v_{i j}-r_{i j}^{*}\right), \forall k \in \mathcal{N} .
\end{aligned}
$$

Figure 3-10: Calculation of adapted allocations and payments. 


\begin{tabular}{|c|c|c|c|c|c|}
\hline $\mathrm{n} / \mathrm{m}$ & 10 & 20 & 30 & 40 & 50 \\
\hline \hline 20 & 4.2 & 5.5 & 8.6 & 10.2 & 12.1 \\
\hline 40 & 5.6 & 9.1 & 11.0 & 16.0 & 15.6 \\
\hline 60 & 7.7 & 13.5 & 18.1 & 20.7 & 24.7 \\
\hline 80 & 11.1 & 14.6 & 20.2 & 26.2 & 30.6 \\
\hline 100 & 10.6 & 17.9 & 26.6 & 34.8 & 45.3 \\
\hline
\end{tabular}

Table 3.5: Computational times (in minutes) of ROM-Si for uncorrelated valuations.

\begin{tabular}{|c|c|c|c|c|c|}
\hline $\mathrm{n} / \mathrm{m}$ & 10 & 20 & 30 & 40 & 50 \\
\hline \hline 20 & 6.04 & 8.5 & 12.3 & 16.3 & 16.3 \\
\hline 40 & 9.9 & 15.4 & 19.5 & 23.4 & 23.4 \\
\hline 60 & 11.02 & 21.2 & 28.0 & 32.1 & 35.7 \\
\hline 80 & 16.2 & 24.9 & 33.4 & 36.2 & 51.0 \\
\hline 100 & 17.6 & 28.7 & 39.0 & 53.2 & 64.3 \\
\hline
\end{tabular}

Table 3.6: Computational times (in minutes) of ROM-Si for correlated valuations with correlation coefficient equal to 0.2 .

(a) the computational tractability of $R O M$ in multi-item auctions with budgets.

(b) the benefits of incorporating correlations in valuations in the design of auctions using $R O M$.

\subsubsection{Computational Tractability}

The computationally intensive step in $R O M$ is to solve the bilinear optimization problem (3.8). We have implemented the algorithm presented in Figure 3-3. In this section, we want to assess the running times of the ROM with uncertainty sets $\mathcal{U}^{\text {CLT }}$ and $\mathcal{U}^{\text {Corr }}$ as $n, m$ and the correlation coefficients vary. In Tables $3.5,3.6$, and 3.7, we report the average computational times in terms of the number of iterations it took to solve the problem for (a) uncorrelated valuations, (b) correlated valuations with correlation coefficient 0.2 , and (c) correlated valuations with correlation coefficient 0.4 .

From the tables, we observe that the running times are insensitive to the correlation coefficient, while growing approximately linearly in $n, m$. We feel that these results indicate that $R O M$ is computationally tractable even for large scale market 


\begin{tabular}{|c|c|c|c|c|c|}
\hline $\mathrm{n} / \mathrm{m}$ & 10 & 20 & 30 & 40 & 50 \\
\hline \hline 20 & 6.53 & 9.2 & 13.3 & 17.4 & 17.4 \\
\hline 40 & 10.2 & 15.6 & 19.4 & 24.4 & 34.3 \\
\hline 60 & 12.8 & 19.9 & 28.8 & 33.9 & 39.2 \\
\hline 80 & 18.3 & 29.9 & 32.7 & 43.2 & 52.6 \\
\hline 100 & 20.1 & 33.1 & 41.95 & 60.16 & 72.5 \\
\hline
\end{tabular}

Table 3.7: Computational times (in minutes) of ROM-Si for correlated valuations with coefficient equal to 0.4 .

design problems.

\subsubsection{Effect of Correlations on the Revenue}

In this experiment, we demonstrate how incorporating correlation information can lead to revenue gains. We compare the revenue of the Myerson auction, which ignores any correlation information, with the revenue obtained by using $R O M$ with the uncertainty set $\mathcal{U}^{\text {Corr }}$ (see Eq.1.3). We compute the Relative Revenue as defined in (3.67) and plot it against the value of correlation in Figure 3-11. We observe that there are significant benefits in incorporating correlation information, which $R O M$ allows us to do.

\subsection{Summary and Conclusions}

In this chapter, we revisited the auction design problem for multi-item auctions with budget constrained buyers by considering a novel modeling approach to model the beliefs of the auctioneer. Instead of using probability distributions to model valuations, or considering a completely adversarial model as in the earlier literature, we consider an uncertainty set based model for the auctioneer's beliefs about the valuations of the buyers. These uncertainty sets can be constructed to incorporate any historical or moment information available to the auctioneer. We then considered the problem of designing an auction that maximizes the worst case revenue assuming the valuations lie in the uncertainty set constructed. For such a model and the objective function considered, we provided a characterization of the worst-case optimal auction for auc- 


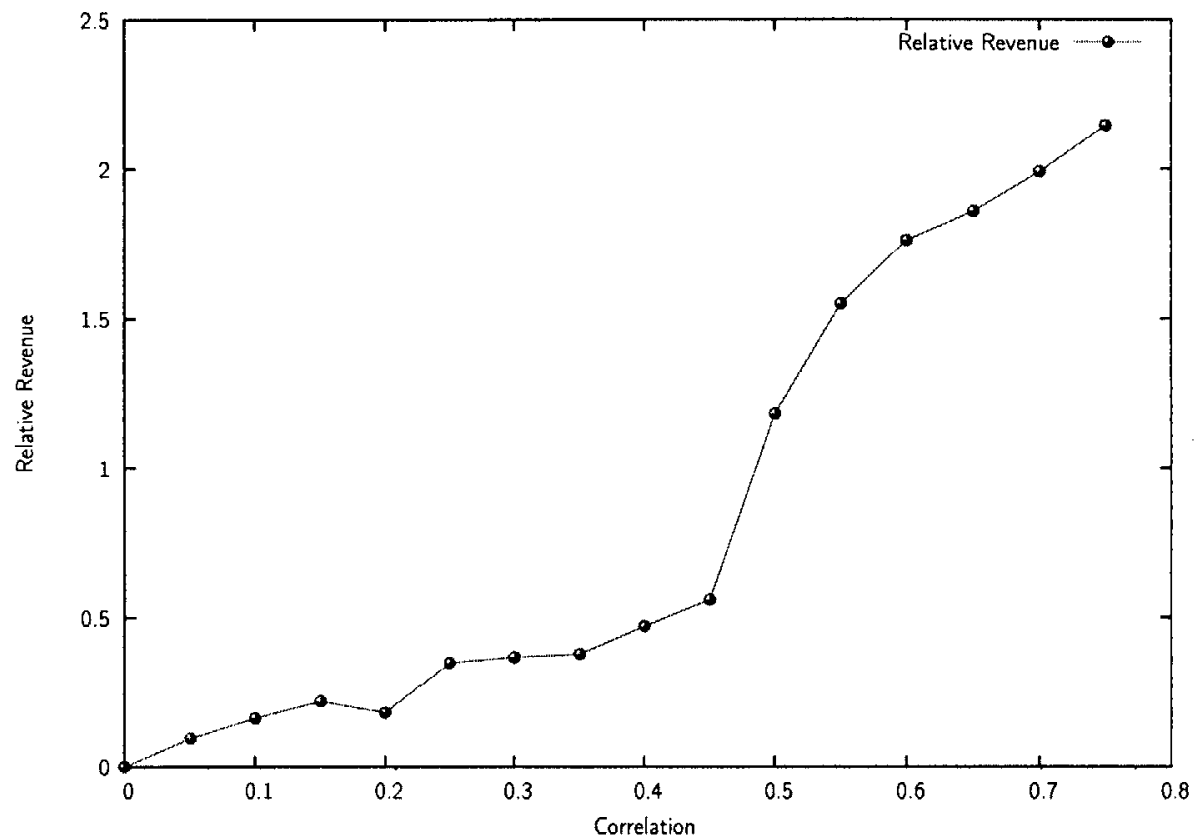

Figure 3-11: Effect of Correlations on the Revenue.

tioning multi-items to multiple budgeted buyers. We further considered extensions of this problem for indivisible items and for buyers with uncertain budgets and provided mechanisms for these extensions.

The key implications of our results are:

1. The optimal auction has reservation prices that are functions of the uncertainty set and the budgets, thus extending the structure of the Myerson auction from single item auctions without budget constraints, to multiple item auctions with budgets, potentially correlated valuations and uncertain budgets.

2. Unlike the Myerson auction where the reservation prices do not depend on the item, the reservation prices in our approach are a function of both the bidder and the item.

3. The mechanism calculates the reservation prices by solving a bilinear optimization problem which, although theoretically difficult in general, is numerically tractable. We presented empirical evidence displaying the numerical tractability 
of our approach.

4. The uncertainty set approach can be used to capture correlations in the buyer valuations and can also model possible uncertainties in the budgets. We showed that the nature of the worst case optimal policy does not change with these extensions.

5. Given the numerical tractability of our approach, it is possible to derive insights on the properties of the optimal policies. We feel that the two most important insights are (a) the proposed approach has attractive robustness properties, when the distributions or the standard deviations of the valuations are misspecified, and (b) the knowledge of correlations can be exploited to yield higher revenue in the auction. 


\section{Chapter 4}

\section{Network Information Theory}

\subsection{Introduction}

The central problem of communications is how to transmit information reliably through a noisy (and thus unreliable) communication channel. Shannon's "A mathematical theory of communication" published in 1948 marks the beginning of Information Theory (Verdu [1998], Verdu and McLaughlin [1998], Cover and Thomas [2006]) and posed the channel coding problem for the first time. In this seminal paper he developed a framework to precisely define the intuitive notion of information which in turn makes it possible to mathematically capture the notions of a communication channel and its capacity. This framework relied heavily on using probabilistic models to describe various components of the communication system. The information source as well as the communication channel were both modeled by using probability distributions. In this framework, Shannon showed (a) there is an upper bound (the so called channel capacity) for the rate of reliable transmission of information, and (b) there exists a code that leads to a rate of transmission arbitrarily close to the channel capacity achieving probabilities of error arbitrarily close to zero. Since then, Shannon's approach has been used to characterize the fundamental limits of communication for various kinds of channels. However, the communication limits on many common channels such as the interference and the multi-cast channels still remain unknown. And indeed a general theory for communication limits on networks of channels is still 
largely open. Techniques such as random encoding that are effective for single user channels no longer allow us to characterize the capacity regions of complex channels, and there is a need to develop widely extendable and structured ways of constructing the codes.

In this chapter, we propose a methodology that in a wide variety of communication channels, is capable of (a) characterizing the capacity regions, and (b) at the same time constructs matching optimal codes. Our approach, which is based on robust optimization, leads to solving a semidefinite optimization problem with rank one constraints which we solve, using existing algorithms, as a sequence of regular semidefinite optimization problems. As an illustration of computational tractability, we report the exact capacity region for a two-transmitter two-receiver interference channel, and a multi-cast channel with two receivers, for message-book sizes of up to 100,000 . As expected, our capacity regions are within the best known lower and upper bounds of the capacity limits reported in literature (see Verdu and McLaughlin [1998]).

In what follows, we briefly describe the key ingredients of our approach:

(1) Identifying the optimal decoder: The first step involves the identification of an optimal decoder for a given communication channel. For single-user channels, it is well known that the Maximum Likelihood decoder is an optimal decoder. For multi-user channels such as the interference and multi-cast channels, we construct new optimal decoders.

(2) Typical sets as uncertainty sets: We used the original idea of Shannon [1948a], that for the purpose of characterizing the capacity region and constructing the underlying code, it suffices to only consider noise sequences that belong to the so called "typical set". We next interpret these typical sets as uncertainty sets in a robust optimization setting. Specifically, we require that the decoding constraints found in Step 1, hold for all noise sequences in the typical set. With this interpretation, the underlying decoding constraints constitute a robust optimization problem. 
(3) Reformulation of the robust optimization problem: We reformulate the underlying robust optimization problem as either a binary linear optimization problem (for non-Gaussian channels) or a non-convex quadratic optimization problem (for Gaussian channels). For the case of Gaussian channels, we reformulate the non-convex quadratic optimization problem as a semidefinite optimization problem with rank constraints. We then use the log-Det method developed in Fazell et al. [2003] to solve this problem as a sequence of regular semidefinite optimization problems.

We feel that the identification of optimal decoders for multi-user channels in Step 1 and formulating the coding problem as a robust optimization problem in Step 2, are decisively new elements in our approach.

\subsubsection{Problem Definition and Notation}

Throughout the chapter, we denote scalar quantities by non-bold face symbols (e.g., $x \in \mathbb{R}, k \in \mathbb{N}$ ), vector quantities by boldface symbols (e.g., $\mathbf{x} \in \mathbb{R}^{n}, n>1$ ), and matrices by uppercase boldface symbols (e.g., $\mathbf{A} \in \mathbb{R}^{n \times m}, n>1, m>1$ ). We denote scalar random variables as $\tilde{z}$ and vector random variables as $\tilde{\mathbf{z}}$. We use the notation from Cover and Thomas [2006] for common information theoretic objects.

To make the chapter self-contained, we define the notion of a communication channel and the related channel coding problem. A communication channel is a medium on which users send and receive messages. For example, in a single user Gaussian channel, a sender transmits signal $x_{j} \in \mathbb{R}$, but the receiver receives

$$
y_{j}=x_{j}+\tilde{z}_{j}, \forall j=1, \ldots, n
$$

where the noise $\tilde{z}_{j}$ is distributed according to the normal distribution $N(0, \sigma)$. In this chapter we will use $\tilde{\mathbf{z}}_{G}$ to denote Gaussian noise and use $\tilde{\mathbf{z}}_{G} \sim N(\mathbf{0}, \sigma \cdot \boldsymbol{e})$ to denote that each component of $\tilde{\mathbf{z}}_{G}$ is normally distributed with mean 0 and standard deviation .

Given a communication channel, a sender seeks to transmit messages from a 
message-book $\mathcal{M}$ by coding the messages using codewords of length $n$, according to a code $\mathcal{C}$. The inputs of such a code $\mathcal{C}$ are:

(a) The length $n$ of the codewords.

(b) The number $M=|\mathcal{M}|=2^{n R}$ of codewords. The quantity $R=\frac{1}{n} \log _{2} M$ is called the rate of the code.

(c) The noise standard deviation $\sigma$.

(d) The power constraint $P$ of the sender.

math The average probability of error $\epsilon>0$ (see Eq. (4.1)) the user tolerates.

The outputs of $\mathcal{C}[n, R, P, \sigma, \epsilon]$ are:

(a) A code-book $\mathcal{B}$, which is a set of $M$ codewords $\mathbf{x}_{i}, \forall i=1, \ldots, M$,

(b) A decoding function $g: \mathbb{R}^{n} \rightarrow\{1,2, \ldots, M\}$ that maps each received word y to one of the codewords in $\mathcal{B}$, while satisfying the error-tolerance of $\epsilon$. That is, for each $i=1, \ldots, M$, we must have

$$
\mathbb{P}\left[g\left(\mathbf{x}_{i}+\tilde{\mathbf{z}}\right) \neq i\right] \leq \epsilon
$$

where $\tilde{\mathbf{z}}=\left[\tilde{z}_{1}, \tilde{z}_{2}, \ldots, \tilde{z}_{n}\right]$, such that $\tilde{z}_{i} \sim N(0, \sigma)$.

The capacity region of a single user Gaussian channel $\mathcal{R}_{n}[P, \sigma, \epsilon]$ is the set of all rates $R$ such that there exists a code $\mathcal{C}[n, R, P, \sigma, \epsilon]$. In the next section, we present a brief review of the information theory literature organized around the results on different channels.

\subsubsection{Relevant Literature}

\section{Single User Channel}

For the case of a single user communication channel, Shannon [1948a] showed that there exists a maximum rate $R_{\max }$ associated with every communication channel, 
above which no reliable transmission is possible and below which there exists a code achieving small error probabilities. In particular, Shannon showed that $R_{\max }$ is given by

$$
R_{\max }=\sup I(X, Y)
$$

where $I(X, Y)$ is the mutual information between the random variables $X$ and $Y$, and the supremum is with respect to all possible information sources used as inputs to the channel. He showed that arbitrarily small probability of errors can be achieved by using random encoding with maximum likelihood decoding, whenever the rate of transmission is less than $R_{\max }$. For the case of the Gaussian channel where the noise is normally distributed with mean 0 and standard deviation $\sigma$, Shannon obtained that the capacity of the channel is

$$
R_{\max }^{\mathrm{Gaussian}}=\frac{1}{2} \log \left(1+\frac{P}{\sigma^{2}}\right)
$$

where $P$ represents the power constraints of the transmitter.

\section{Multi-User Channels}

(1) Multi-access Channels : Gamal and Cover [1980] found various characterizations of the capacity region of the two-user discrete memoryless multi-access channel. Most useful among those is the expression found by Carleial [1975] for the capacity region as the convex hull of a union of pentagons. Wyner [1967] showed (using the suboptimal successive cancellation decoder) that the memoryless Gaussian multiple-access channel admits a very simple capacity region: the pentagon defined by the single-user capacities of the channels with powers equal to the individual powers and to the sum of the powers.

(2) Interference Channels : In contrast to the multi-access setting in which the receiver is interested in decoding the information sent by all the users, suppose now that we have as many receivers as transmitters and each receiver is interested in decoding only one of the sources. In spite of many efforts surveyed 
in Verdu and McLaughlin [1998], the capacity region of even the simplest twouser memoryless Gaussian interference channel remains an open problem. The only case in which the capacity is known is in the strong interference case, where each receiver has a better reception of the other user's signal than the intended receiver (Sason [2004]). The best known strategy for more general cases is due to Han and Kobayashi [1981]. Etkin et al. [2008] show that a simple Han-Kobayashi type scheme can in fact achieve rates within $1 \mathrm{bit} / \mathrm{s} / \mathrm{Hz}$ of the capacity of the channel for all values of the channel parameters.

(3) Broadcast Channels : Although a general solution for the capacity region of the broadcast channel is not yet known, considerable progress (surveyed in Verdu [1998]) has been made in exploring the fundamental limits of various classes of memoryless broadcast channels. However, the main problem remains open.

\subsubsection{Structure of the chapter}

The rest of the chapter is organized as follows. In Section 4.2, we review two key ideas: maximum likelihood decoding and typical sets. Furthermore, we construct typical sets for general pdfs $f(\cdot)$. Subsequently, we present the characterization of the capacity region and corresponding optimal codes as follows:

- single user Gaussian channel in Section 4.3,

- two-user interference channel in Section 4.4,

- multi-cast and multi-access channels in Section 4.5,

- single user and multi-user channels with exponentially distributed noise in Section 4.6,

In Section 4.7, we outline the semidefinite optimization approach we use in Gaussian channels (Sections 4.3, 4.4, 4.5) and present computational results for two-user interference, multi-cast and multi-access channels. In Section 4.8, we present some concluding remarks. 


\subsection{Maximum Likelihood Decoding and Typical Sets}

In this section, we review two key ideas we use in our approach: the idea that maximum likelihood decoding is optimal and the notion of a typical set. We consider a single user channel in which the noise is distributed according a pdf $f(\cdot)$.

\subsubsection{Optimality of Maximum Likelihood Decoder}

The Maximum Likelihood (ML) decoder is an optimal decoder for any single user channel (see Cover and Thomas [2006]), that is, there always exists an optimal code which uses ML as the decoding function. An ML decoder is characterized by the decoding function $g^{\mathrm{ML}}(\cdot)$ given by

$$
\begin{aligned}
g^{\mathrm{ML}}(\mathbf{y}) & =\arg \max _{\mathbf{x}_{i} \in \mathcal{B}} \mathbb{P}\left[\mathbf{y} \mid \mathbf{x}_{i} \text { was sent }\right] \\
& =\arg \max _{\mathbf{x}_{i} \in \mathcal{B}} \prod_{j=1}^{n} f\left(y_{j}-x_{i j}\right)
\end{aligned}
$$

This allows us to formulate the coding problem as an optimization problem by restricting our attention to codes that are optimal with respect to the ML decoder. In particular, we constrain the codewords to satisfy the constraints:

$$
\begin{aligned}
\mathbf{x}_{i} & \in \mathcal{X}^{n}, \forall i=1, \ldots, M \\
\prod_{j=1}^{n} f\left(x_{i j}+z_{j}-x_{i^{\prime} j}\right) & \geq \prod_{j=1}^{n} f\left(z_{j}\right), \forall i, i^{\prime} \neq i, \forall \mathbf{z} \in \mathcal{U},
\end{aligned}
$$

where $\mathcal{U}$ is an appropriately chosen uncertainty set such that

$$
\mathbb{P}[\tilde{\mathbf{z}} \in \mathcal{U}] \geq 1-\epsilon
$$

Note that Eq. (4.4) are expressed in the language of robust optimization. In particular, this is an example where robustness is inherent in the problem. That is robustness 
enters the problem, not as a way of modeling uncertainty, but as a natural property of the problem.

If the codewords are feasible to (4.3) and (4.4), then it is clear to see that the maximum error probability $\mathbb{P}_{\text {error }}$ is constrained by

$$
\begin{aligned}
\mathbb{P}_{\text {error }} & \leq \mathbb{P}[\tilde{\mathbf{z}} \notin \mathcal{U}] \\
& =1-\mathbb{P}[\tilde{\mathbf{z}} \in \mathcal{U}] \\
& =\epsilon .
\end{aligned}
$$

Note that there are multiple $\mathcal{U}$ mathnormaltpical isfy (4.5), therefore, the problem now reduces to identifying the set $\mathcal{U}$ that satisfies (4.5), while also allowing us to characterize the capacity region using this optimization approach. We show that this indeed can be done by choosing $\mathcal{U}$ to be the so called typical set, which was introduced in Chapter 1, Eq. (1.6).

\subsection{The Single User Gaussian Channel}

We consider here a discrete-time memoryless additive Gaussian channel being used by a single user with average power constraint on the transmitted codewords. Each use of the channel involves transmitting a signal $x \in \mathbb{R}$, which is transformed by the channel into a signal $y \in \mathbb{R}$ according to

$$
y=x+\tilde{z}
$$

where the noise $\tilde{z}$ is drawn independently from a Gaussian distribution with mean 0 and standard deviation $\sigma$, and is also assumed to be independent of the signal $x$. The codewords are subject to an average power constraint $P$, that is, for any codeword $\mathbf{x}_{i} \in \mathcal{B}$ we require that

$$
\left\|\mathbf{x}_{i}\right\|^{2} \leq n P
$$


Shannon [1948a] obtained the channel coding theorem that asserts the existence of codes with rates arbitrarily close to the channel capacity and probabilities of error arbitrarily close to zero. The proof used the technique of random encoding and maximum likelihood decoding, to show that the error probability averaged with respect to the codebook choice vanishes asymptotically with large code lengths whenever the transmission rate is less than the channel capacity. Subsequently Feinstein [1954] was able to obtain an alternate proof of the coding theorem without using the random encoding technique, although the decoder was again based on the idea of typicality. Shannon was able to obtain a closed form characterization of the capacity region, as presented in the following theorem.

Theorem 26 (Shannon [1948a]). In a single-user communication channel with power constraint $P$ and normally distributed noise with mean 0 and standard deviation $\sigma$, the asymptotic channel capacity $R_{\max }$ is given by

$$
R_{\max }=\frac{1}{2} \log \left(1+\frac{P}{\sigma^{2}}\right)
$$

That is, the user can transmit at any rate $R<R_{\max }$ with vanishing decoding errorprobabilities as $n \rightarrow \infty$, while for any rate $R>R_{\max }$, the error-probabilities tend to 1 , as $n \rightarrow \infty$.

\subsubsection{An Optimization Formulation of the Coding Problem}

In this section, we present an optimization formulation of the channel coding problem. We begin by observing that the maximum likelihood decoder for a single user Gaussian channel is the minimum distance decoder given by

$$
\left\|\mathbf{x}_{i}+\mathbf{z}-\mathbf{x}_{i^{\prime}}\right\| \geq\|\mathbf{z}\| \quad \forall \mathbf{z} \in \mathcal{U}_{\epsilon}^{\text {Normal }}, \forall i, i^{\prime} \neq i
$$


where $\mathcal{U}_{\epsilon}^{\text {Normal }}$ is the typical set for the Normal distribution (see Eq. 1.5). These constraints ensure that, if $\mathbf{x}_{i}$ was transmitted by the user and was received as

$$
\mathbf{y}=\mathbf{x}_{i}+\mathbf{z} \text { for some } \mathbf{z} \in \mathcal{U}_{\epsilon}^{\text {Normal }}
$$

then the distance between the received codeword $\mathbf{y}$ and any other codeword $\mathbf{x}_{i^{\prime}}$ is greater than the distance between $\mathbf{y}$ and $\mathbf{x}_{i}$. Motivated by this, we next present a robust optimization feasibility problem (see Eqs. (4.94)-(4.99)) that allows us to characterize the capacity region of a single user Gaussian channel. In particular, the inputs to the feasibility problem in (4.94)-(4.99) are:

(a) The length $n$ of the codewords;

(b) The rate $R$ of the code;

(c) The standard deviation $\sigma$ of the normally distributed noise;

(d) The power constraint $P$ of the user;

(e) The maximum probability of error $\epsilon>0$ (see Eq. (4.1)) the user tolerates;

(f) The parameter $\nu>0$ that controls how close (4.18) characterizes the capacity region.

Given these inputs, we first calculate the following "derivative" quantities:

(a) The parameter $\gamma_{\epsilon}$, which we choose so that

$$
\mathbb{P}\left[\left\|\tilde{\mathbf{z}}_{G}\right\| \leq \gamma_{\epsilon}\right] \geq 1-\epsilon
$$

where $\tilde{\mathbf{z}}_{G} \sim N(\mathbf{0}, \sigma \cdot \boldsymbol{e})$

(b) The parameter $T$ given by

$$
T=\left(\frac{1+\nu}{\zeta \nu} \cdot \frac{\gamma_{\epsilon}}{\sqrt{n}}\right)^{n}, \text { with } \zeta=\frac{\sigma}{\sqrt{n}} \cdot \Phi^{-1}(1-\epsilon(1-\delta(\nu, n)))
$$


where $\Phi(\cdot)$ is the cdf of a standard normal and

$$
\delta(\nu, n)=\exp \left(-n \cdot \frac{r-\log (1+r)}{2(1+3 \nu)^{2} \sigma^{2}}\right), \text { with } r=\sigma^{2}\left((1+3 \nu)^{2}-(1+2 \nu)^{2}\right)
$$

(c) The parameter $M_{0}=(1+\nu) \cdot \gamma_{\epsilon}$.

(d) The set of vectors $\mathcal{Z}=\left\{\mathbf{z}_{1}, \mathbf{z}_{2}, \ldots, \mathbf{z}_{T}\right\}$ with $\left\|\mathbf{z}_{t}\right\|=M_{0}, t=1, \ldots, T$, and the property that the Voronoi regions induced by $\mathbf{z}_{t}$ are identical geometrically. The Voronoi region induced by $\mathbf{z}_{t}$ is the set of all points on $\mathcal{S}_{n}\left(M_{0}\right)$ closest to $\mathbf{z}_{i}$, i.e.,

$$
\mathcal{V}\left(\mathbf{z}_{t}\right)=\left\{\mathbf{z} \in \mathcal{S}_{n}\left(M_{0}\right) \mid\left\|\mathbf{z}_{t}-\mathbf{z}\right\| \leq\left\|\mathbf{z}_{s}-\mathbf{z}\right\|, \forall s \neq t\right\}
$$

These vectors are the deterministic equivalent to being uniformly distributed on the surface of $\mathcal{S}_{n}\left(M_{0}\right)$. The construction of such vectors is given in Wyner [1967].

We define decision variables $\mathbf{x}_{i}, \forall i=1, \ldots, 2^{n R}$ and $v_{i t}, \forall i=1, \ldots, 2^{n R}, \forall t=1, \ldots, T$, where

1. The variables $\mathbf{x}_{i}$ represent the codewords.

2. The variables $v_{i t}$ represent binary decision variables that are chosen in a way to constrain the probability of error. When $v_{i t}=1$, the set of decoding constraints (4.11) are satisfied for codeword $\mathbf{x}_{i}$ with noise vector $\mathbf{z}_{t}$. 
We consider the following feasibility problem:

$$
\begin{array}{cl}
\left\|\mathbf{x}_{i}\right\|^{2} \leq n P & \forall i=1, \ldots, 2^{n R}, \\
\left\|\mathbf{x}_{i}-\mathbf{x}_{k}+\mathbf{z}_{t}\right\|+\left(1-v_{i t}\right) M_{0} \geq\left\|\mathbf{z}_{t}\right\|, & \forall t, \forall i, k \neq i, \\
\left\|\mathbf{x}_{i}-\mathbf{x}_{k}\right\| \geq 2 \zeta \sqrt{n}, & \forall i, k \neq i, \\
\sum_{t=1}^{T} v_{i t} \geq(1-\epsilon) T, & \forall i, \\
v_{i t} \in\{0,1\}, & \forall i, t,
\end{array}
$$

Constraints (4.9) impose power constraints on the codewords. Constraints (4.11), (4.14) ensure that each codeword $\mathbf{x}_{i}$ is decoded correctly for at least $(1-\epsilon) T$ of the noise vectors. Constraint (4.13) ensures that the codewords are separated by a certain minimum distance to obtain a decoding error probability of at most $\epsilon$.

We next reformulate the feasibility problem (4.9)-(4.15) as a semidefinite optimization problem with rank constraints, by using the following proposition.

\section{Proposition 27.}

(a) Constraint $\left\|\mathbf{x}_{i}-\mathbf{x}_{k}+\mathbf{z}_{t}\right\|+\left(1-v_{i t}\right) M_{0} \geq\left\|\mathbf{z}_{t}\right\|, \forall t=1, \ldots, T, \forall i, k \neq i$, is equivalent to the constraint

$$
\left\|\mathbf{x}_{i}-\mathbf{x}_{k}\right\|^{2}+M_{0}^{2}\left(1-v_{i t}\right) \geq 2\left(\mathbf{x}_{k}-\mathbf{x}_{i}\right)^{\prime} \mathbf{z}_{t}, \forall t=1, \ldots, T, \forall i, k \neq i
$$

(b) Constraint $v_{i t} \in\{0,1\}$ is equivalent to the constraint $v_{i t}^{2}=v_{i t}$. 
Proof. (a) When $v_{i t}=1$,

$$
\begin{aligned}
& \left\|\mathbf{x}_{i}-\mathbf{x}_{k}+\mathbf{z}_{t}\right\|+\left(1-v_{i t}\right) M_{0} \geq\left\|\mathbf{z}_{t}\right\| \\
\Longleftrightarrow & \left\|\mathbf{x}_{i}-\mathbf{x}_{k}+\mathbf{z}_{t}\right\| \geq\left\|\mathbf{z}_{t}\right\| \\
\Longleftrightarrow & \left\|\mathbf{x}_{i}-\mathbf{x}_{k}+\mathbf{z}_{t}\right\|^{2} \geq\left\|\mathbf{z}_{t}\right\|^{2} \\
\Longleftrightarrow & \left\|\mathbf{x}_{i}-\mathbf{x}_{k}\right\|^{2} \geq 2\left(\mathbf{x}_{k}-\mathbf{x}_{i}\right)^{\prime} \mathbf{z}_{t} .
\end{aligned}
$$

On the other hand, when $v_{i t}=0$,

$$
\begin{aligned}
& \left\|\mathbf{x}_{i}-\mathbf{x}_{k}+\mathbf{z}_{t}\right\|+\left(1-v_{i t}\right) M_{0} \geq\left\|\mathbf{z}_{t}\right\| \\
\Longleftrightarrow & \left\|\mathbf{x}_{i}-\mathbf{x}_{k}+\mathbf{z}_{t}\right\| \geq\left\|\mathbf{z}_{t}\right\|-M_{0} \\
\Longleftrightarrow & \left\|\mathbf{x}_{i}-\mathbf{x}_{k}+\mathbf{z}_{t}\right\|^{2} \geq\left\|\mathbf{z}_{t}\right\|^{2}+M_{0}^{2}-2 M_{0}\left\|\mathbf{z}_{t}\right\| \\
\Longleftrightarrow & \left\|\mathbf{x}_{i}-\mathbf{x}_{k}\right\|^{2}+M_{0}^{2} \geq 2\left(\mathbf{x}_{k}-\mathbf{x}_{i}\right)^{\prime} \mathbf{z}_{t},
\end{aligned}
$$

where the last equivalence follows from $\left\|\mathbf{z}_{t}\right\|=M_{0}$. Therefore,

$$
\begin{array}{r}
\left\{\left\|\mathbf{x}_{i}-\mathbf{x}_{k}+\mathbf{z}_{t}\right\|+\left(1-v_{i t}\right) M_{0} \geq\left\|\mathbf{z}_{t}\right\|\right\} \\
\Longleftrightarrow\left\{\left\|\mathbf{x}_{i}-\mathbf{x}_{k}\right\|^{2}+M_{0}^{2}\left(1-v_{i t}\right) \geq 2\left(\mathbf{x}_{k}-\mathbf{x}_{i}\right)^{\prime} \mathbf{z}_{t}\right\} .
\end{array}
$$

(b) We have that $v_{i t} \in\{0,1\}$ if and only if $v_{i t}^{2}=v_{i t}$.

Using Proposition 27, we convert the feasibility problem (4.94)-(4.99) to a nonconvex quadratic optimization problem.

Proposition 28. The set of $K$ quadratic (possibly non-convex) constraints

$$
f_{k}(\mathbf{y})=\mathbf{y}^{\prime} A_{k} \mathbf{y}+\mathbf{b}_{k}^{\prime} \mathbf{y}+c_{k} \leq 0, \forall k=1, \ldots, K
$$


is equivalent to the semidefinite optimization problem

$$
\begin{gathered}
\tilde{A}_{k} \bullet Y \leq 0, \quad \forall k=1, \ldots, K, \\
Y \succeq 0 \\
Y_{11}=1, \\
\operatorname{rank}(Y)=1,
\end{gathered}
$$

where

$$
\begin{aligned}
Y & =\left(\begin{array}{l}
1 \\
\mathbf{y}
\end{array}\right)\left(1, \mathbf{y}^{\prime}\right), \\
\tilde{A}_{k} & =\left(\begin{array}{cc}
c_{k} & \mathbf{b}_{k} \\
\mathbf{b}_{k} & A_{k}
\end{array}\right) .
\end{aligned}
$$

Proof. The quadratic function $f_{k}(\cdot)$ can be written as

$$
f_{k}(\mathbf{y})=\left(1, \mathbf{y}^{\prime}\right)\left(\begin{array}{cc}
c_{k} & \mathbf{b}_{k} \\
\mathbf{b}_{k} & A_{k}
\end{array}\right)\left(\begin{array}{c}
1 \\
\mathbf{y}
\end{array}\right)=\tilde{A}_{k} \bullet Y
$$

where $Y=\left(\begin{array}{c}1 \\ \mathbf{y}\end{array}\right)\left(1, \mathbf{y}^{\prime}\right)$, and $\tilde{A}_{k}=\left(\begin{array}{cc}c_{k} & \mathbf{b}_{k} \\ \mathbf{b}_{k} & A_{k}\end{array}\right)$. Clearly

$$
Y_{11}=1, Y \succeq 0, \text { and } \operatorname{rank}(Y)=1
$$

In addition, $\tilde{A}_{k} \bullet Y=f_{k}(\mathbf{y}) \leq 0, \forall k=1, \ldots, K$.

On the other hand, given a feasible solution $Y$ to $(4.17)$, because $\operatorname{rank}(Y)=1$ and $Y_{11}=1$, there exists a vector $\mathrm{y}$ such that

$$
Y=\left(\begin{array}{l}
1 \\
\mathbf{y}
\end{array}\right)\left(1, \mathbf{y}^{\prime}\right)
$$


and clearly $\mathbf{y}$ is feasible to (4.16). This concludes the proof.

Using Propositions 27 and 28, we obtain that the feasibility problem (4.94)-(4.99) is equivalent to the semidefinite optimization problem (4.18).

$$
\begin{array}{ll}
\min & \operatorname{rank}(Y) \\
\text { s.t. } & A_{i} \bullet Y \leq 0, \quad \forall i=1, \ldots, 2^{n R}, \\
& B_{i k t} \bullet Y \leq 0, \quad \forall t=1, \ldots, T, \forall i, k \neq i, \\
& C_{i} \bullet Y \leq 0, \quad \forall i \\
& D_{i t} \bullet Y=0, \quad \forall i, t \\
& Y \succeq 0
\end{array}
$$


where

$$
\begin{aligned}
& A_{i}(p, q)= \begin{cases}-n P, & \text { if } p=1, q=1 \\
0, & \text { if } p=1, q>1 \\
0, & \text { if } p>1, q=1 \\
1, & \text { if } \forall p=q=(i-1) n+1, \ldots, i n+1, \\
0, & \text { otherwise. }\end{cases} \\
& B_{i k t}(p, q)= \begin{cases}-M_{0}^{2}, & \text { if } p=1, q=1, \\
z_{t r}, & \text { if } p=1, q=(k-1) n+1+r, \forall r=1, \ldots, n, \\
z_{t r}, & \text { if } q=1, p=(k-1) n+1+r, \forall r=1, \ldots, n, \\
-z_{t r}, & \text { if } p=1, q=(i-1) n+1+r, \forall r=1, \ldots, n, \\
-z_{t r}, & \text { if } q=1, p=(i-1) n+1+r, \forall r=1, \ldots, n, \\
-1, & \text { if } \forall p=q=(i-1) n+1, \ldots, i n+1, \\
-1, & \text { if } \forall p=q=(k-1) n+1, \ldots, k n+1, \\
1, & \text { if } q=(k-1) n+1+r, p=(i-1) n+1+r, \forall r=1, \ldots, n, \\
1, & \text { if } q=(i-1) n+1+r, p=(k-1) n+1+r, \forall r=1, \ldots, n, \\
\frac{M_{0}^{2}}{2} & \text { if } p=1, q=n^{2}+1+(i-1) T+t, \\
\frac{M_{0}^{2}}{2} & \text { if } q=1, p=n^{2}+1+(i-1) T+t, \\
0, & \text { otherwise. }\end{cases} \\
& C_{i}(p, q)= \begin{cases}(1-\epsilon) T, & \text { if } p=1, q=1, \\
-1, & \text { if } \forall p=q=n^{2}+1+(i-1) T+t, \forall t=1, \ldots, T \\
0, & \text { otherwise. }\end{cases} \\
& D_{i t}(p, q)= \begin{cases}0, & \text { if } p=1, q=1 \\
-\frac{1}{2}, & \text { if } p=1, q=n^{2}+1+(i-1) T+t, \\
-\frac{1}{2}, & \text { if } q=1, p=n^{2}+1+(i-1) T+t, \\
1, & \text { if } \forall p=q=n^{2}+1+(i-1) T+t \\
0, & \text { otherwise. }\end{cases}
\end{aligned}
$$


We summarize the development by introducing the following algorithm to characterize the capacity and determine the optimal code.

Algorithm 1 Capacity Characterization and Optimal Coding for the Single User Gaussian Channel.

Input : $R, P, \sigma, n, \nu, \epsilon$.

Output : Rank $r^{*}$, codewords $\left\{\mathbf{x}_{i}\right\}_{i=1}^{2^{n R}}$, and auxiliary binary variables $\left\{v_{i t}\right\}_{t=1}^{T}$.

\section{Algorithm :}

1. Solve the rank minimization semidefinite optimization problem (4.18) to compute $r^{*}$, codewords $\left\{\mathbf{x}_{i}\right\}_{i=1}^{2^{n R}}$, and auxiliary binary variables $\left\{v_{i t}\right\}_{t=1}^{T}$.

2. If $r^{*}=1$, then $R$ is achievable using the codebook $\mathcal{B}=\left\{\mathbf{x}_{i}\right\}_{i=1}^{2^{n R}}$ and the minimum distance decoding function, achieving a decoding error probability of $\epsilon$. That is,

$$
\text { If } r^{*}=1 \text {, then } R \in \mathcal{R}_{n}[P, \sigma, 2 \epsilon] \text {. }
$$

3. If $r^{*} \geq 2$, then $R$ cannot be achieved on a single user Gaussian channel with noise standard deviation $(1+2 \nu) \sigma$ with probability of error less than or equal to $\epsilon(1-\delta(\nu, n))$. That is,

$$
\text { If } r^{*} \geq 2 \text {, then } R \notin \mathcal{R}_{n}[P,(1+3 \nu) \sigma, \epsilon(1-\delta(\nu, n))] \text {, }
$$

where

$$
\begin{aligned}
\delta(\nu, n) & =\exp \left(-n \cdot \frac{r-\log (1+r)}{2(1+3 \nu)^{2} \sigma^{2}}\right), \text { with } r=\sigma^{2}\left((1+3 \nu)^{2}-(1+2 \nu)^{2}\right) \\
& \rightarrow 0, \text { as } n \rightarrow \infty
\end{aligned}
$$

\subsubsection{Correctness of Algorithm 1}

We establish the following notation for this section. We let $\mathscr{E}_{i}[\tilde{\mathbf{z}}]$ denote the event that a decoding error occurs when message $i$ is sent on the channel and noise vector 
$\tilde{\mathbf{z}}$ is realized, that is,

$$
\mathscr{E}_{i}[\tilde{\mathbf{z}}]=\left\{\exists k \neq i:\left\|\mathbf{x}_{i}-\mathbf{x}_{k}+\tilde{\mathbf{z}}\right\| \leq\|\tilde{\mathbf{z}}\|\right\}
$$

Let $\mathbf{1}\left\{\mathscr{E}_{i}[\tilde{\mathbf{z}}]\right\}$ denote the indicator random variable corresponding to $\mathscr{E}_{i}[\tilde{\mathbf{z}}]$, that is,

$$
1\left\{\mathscr{E}_{i}[\tilde{\mathbf{z}}]\right\}= \begin{cases}1, & \text { if } \exists k \neq i:\left\|\mathbf{x}_{i}-\mathbf{x}_{k}+\tilde{\mathbf{z}}\right\| \leq\|\tilde{\mathbf{z}}\| \\ 0, & \text { otherwise }\end{cases}
$$

Let $\mathcal{S}_{n}(r)=\left\{\mathbf{s} \in \mathbb{R}^{n} \mid\|\mathbf{s}\|=r\right\}$, and let $\tilde{\mathbf{s}}(r)$ denote a vector chosen uniformly at random in $\mathcal{S}_{n}(r)$. We next review the following two results from the literature which will be integral to the proof of correctness of Algorithm 1. Wyner [1967] showed that a large collection of uniformly random points on a sphere can be used as a proxy for all the points on the sphere.

Proposition 29 (Wyner [1967]). Let $\mathcal{A}=\left\{\mathbf{a}_{1}, \mathbf{a}_{2}, \ldots, \mathbf{a}_{N}\right\}$ be a Voronoi tessellation on $\mathcal{S}_{n}(\gamma)$. Then, $\forall \mathbf{s} \in \mathcal{S}_{n}(\gamma), \exists \mathbf{a}_{i} \in \mathcal{A}$ such that $\left\|\mathbf{s}-\mathbf{a}_{i}\right\| \leq \theta \sqrt{n}$, where $\theta=\frac{\gamma}{N^{\frac{1}{n}} \cdot \sqrt{n}}$.

The following result lists two important properties of a vector of independent normal random variables.

Proposition 30 (see Cover and Thomas [2006]). Let $\tilde{\mathbf{z}}_{G} \sim N(\mathbf{0}, \sigma \cdot \boldsymbol{e})$.

(a) (Bernstein's inequality) The vectors $\tilde{\mathbf{z}}_{G}$ are concentrated in a thin shell of radius $\sigma \sqrt{n}$, that is,

$$
\mathbb{P}\left[\frac{1}{n}\left\|\tilde{\mathbf{z}}_{G}\right\|^{2}>\sigma^{2}-r\right] \geq 1-\exp \left(-n \cdot \frac{r-\log (1+r)}{2 \sigma^{2}}\right)
$$

(b) (Spherical symmetry) The random vector $\tilde{\boldsymbol{u}}=\tilde{\mathbf{z}}_{G} /\left\|\tilde{\mathbf{z}}_{G}\right\|$ is distributed uniformly in $\mathcal{S}_{n}(1)$.

(c) Let $\tilde{d}$ be a random variable distributed identically to the norm of $\tilde{\mathbf{z}}_{G}$, that is, $\tilde{d} \sim\left\|\tilde{\mathbf{z}}_{G}\right\|$. Then, $\tilde{\mathbf{z}}_{G} \sim \tilde{d} \cdot \tilde{\mathbf{s}}_{n}(1)$. 
We next present a series of propositions that form the components of the proof of correctness of Algorithm 1.

Proposition 31. Let $A, B$ and $C$ be three single user channels with noise vectors $\tilde{\boldsymbol{u}}_{A}, \tilde{\boldsymbol{u}}_{B}, \tilde{\mathbf{z}}_{C}$ where $\tilde{\boldsymbol{u}}_{A}, \tilde{\boldsymbol{u}}_{B}$ are distributed uniformly in $\mathcal{S}_{n}\left(r_{A}\right)$ and $\mathcal{S}_{n}\left(r_{B}\right)$, respectively, and $\tilde{\boldsymbol{z}}_{C} \sim N(\mathbf{0}, \gamma \cdot \mathbf{e})$. Let $\left\{\mathbf{x}_{i}\right\}_{i=1}^{M}$ be the set of codewords. Then,

(a) If $r_{A} \geq r_{B}$, then

$$
\mathbb{P}\left[\exists k \neq i:\left\|\mathbf{x}_{i}-\mathbf{x}_{k}+\tilde{u}_{A}\right\| \leq r_{A}\right] \geq \mathbb{P}\left[\exists k \neq i:\left\|\mathbf{x}_{i}-\mathbf{x}_{k}+\tilde{u}_{B}\right\| \leq r_{B}\right]
$$

(b) $\mathbb{P}\left[\exists k \neq i:\left\|\mathbf{x}_{i}-\mathbf{x}_{k}+\tilde{\boldsymbol{u}}_{A}\right\| \leq r_{A}\right] \geq \mathbb{P}\left[\exists k \neq i:\left\|\mathbf{x}_{i}-\mathbf{x}_{k}+\tilde{\boldsymbol{z}}_{C}\right\| \leq\left\|\tilde{\boldsymbol{z}}_{C}\right\| \mid\left\|\tilde{\boldsymbol{z}}_{C}\right\| \leq r_{A}\right]$;

(c) $\mathbb{P}\left[\exists k \neq i:\left\|\mathbf{x}_{i}-\mathbf{x}_{k}+\tilde{\boldsymbol{u}}_{A}\right\| \leq r_{A}\right] \leq \mathbb{P}\left[\exists k \neq i:\left\|\mathbf{x}_{i}-\mathbf{x}_{k}+\tilde{\boldsymbol{z}}_{C}\right\| \leq\left\|\tilde{\boldsymbol{z}}_{C}\right\|\left\|\tilde{\boldsymbol{z}}_{C}\right\|>r_{A}\right]$.

Proof.

(a) We first show that

$$
\text { if }\left\|\mathbf{x}_{i}-\mathbf{x}_{k}+\tilde{\mathbf{z}}\right\| \leq\|\tilde{\mathbf{z}}\|, \text { then }\left\|\mathbf{x}_{i}-\mathbf{x}_{k}+\alpha \tilde{\mathbf{z}}\right\| \leq \alpha\|\tilde{\mathbf{z}}\|, \forall \alpha \geq 1
$$

If $\left\|\mathbf{x}_{i}-\mathbf{x}_{k}+\tilde{\mathbf{z}}\right\| \leq\|\tilde{\mathbf{z}}\|$, then we have

$$
\begin{aligned}
\left\|\mathbf{x}_{i}-\mathbf{x}_{k}+\alpha \tilde{\mathbf{z}}\right\| & =\left\|\mathbf{x}_{i}-\mathbf{x}_{k}+\tilde{\mathbf{z}}+(\alpha-1) \tilde{\mathbf{z}}\right\| \\
& \leq\left\|\mathbf{x}_{i}-\mathbf{x}_{k}+\tilde{\mathbf{z}}\right\|+\|(\alpha-1) \tilde{\mathbf{z}}\| \quad \text { (triangle inequality) } \\
& \leq\|\tilde{\mathbf{z}}\|+(\alpha-1)\|\tilde{\mathbf{z}}\|=\alpha\|\tilde{\mathbf{z}}\| .
\end{aligned}
$$

We next consider a sample path $\omega_{B}$ such that $\exists \tilde{k} \neq i:\left\|\mathbf{x}_{i}-\mathbf{x}_{\tilde{k}}+\tilde{\boldsymbol{u}}_{B}\left(\omega_{B}\right)\right\| \leq r_{B}$. Then, consider a sample path $\omega_{A}$ given by

$$
\tilde{u}_{A}\left(\omega_{A}\right)=\frac{r_{A}}{r_{B}} \cdot \tilde{u}_{B}\left(\omega_{B}\right)
$$

Applying (4.19) with $\alpha=r_{A} / r_{B} \geq 1$, we have $\left\|\mathbf{x}_{i}-\mathbf{x}_{\tilde{k}}+\alpha \tilde{\boldsymbol{u}}_{B}\left(\omega_{B}\right)\right\| \leq \alpha r_{B}$, leading 
to $\left\|\mathbf{x}_{i}-\mathbf{x}_{\tilde{k}}+\tilde{\boldsymbol{u}}_{A}\left(\omega_{A}\right)\right\| \leq r_{A}$. In other words, we have

$$
1\left\{\mathscr{E}_{i}\left[\tilde{\boldsymbol{u}}_{A}\left(\omega_{A}\right)\right]\right\} \geq 1\left\{\mathscr{E}_{i}\left[\tilde{\boldsymbol{u}}_{B}\left(\omega_{B}\right)\right]\right\}
$$

Noting that, if $\tilde{\boldsymbol{u}}_{B}\left(\omega_{B}\right)$ is uniformly distributed in $\mathcal{S}_{n}\left(r_{B}\right)$, then $\tilde{\boldsymbol{u}}_{A}\left(\omega_{A}\right)$ is also uniformly distributed in $\mathcal{S}_{n}\left(r_{A}\right)$, by taking expectations in (4.20), we have

$$
\mathbb{P}\left[\exists k \neq i:\left\|\mathbf{x}_{i}-\mathbf{x}_{k}+\tilde{u}_{A}\right\| \leq r_{A}\right] \geq \mathbb{P}\left[\exists k \neq i:\left\|\mathbf{x}_{i}-\mathbf{x}_{k}+\tilde{u}_{B}\right\| \leq r_{B}\right]
$$

(b) Let $\mathrm{s} \in \mathcal{S}_{n}(1)$, and let $f_{i}, g_{i}: \mathcal{S}^{n}(1) \rightarrow\{0,1\}$ be defined as

$$
\begin{aligned}
& f_{i}(\mathbf{s})= \begin{cases}1, & \text { if } \exists d \leq r_{A}:\left\|\mathbf{x}_{i}-\mathbf{x}_{k}+d \mathbf{s}\right\| \leq d, \text { for some } k \neq i \\
0, & \text { otherwise. }\end{cases} \\
& g_{i}(\mathbf{s})= \begin{cases}1, & \text { if }\left\|\mathbf{x}_{i}-\mathbf{x}_{k}+r_{A} \mathbf{s}\right\| \leq r_{A}, \text { for some } k \neq i, \\
0, & \text { otherwise. }\end{cases}
\end{aligned}
$$

Applying (4.19) with $\tilde{\mathbf{z}}=d \cdot \mathbf{s}$ and $\alpha=r_{A} / d \geq 1$, we have that if $f_{i}(\mathbf{s})=1$, then $g_{i}(\mathbf{s})=1$ leading to

$$
\mathbb{P}_{\mathbf{s} \sim \overline{\mathbf{s}}(1)}\left[g_{i}(\mathbf{s})=1\right] \geq \mathbb{P}_{\mathbf{s} \sim \tilde{\mathbf{s}}(1)}\left[f_{i}(\mathbf{s})=1\right]
$$

We next consider the event $\left\{\mathscr{E}_{i}[d \mathrm{~s}] \mid d=r_{A}\right\}$. We have

$$
\left\{\mathscr{E}_{i}[d \mathbf{s}] \mid d=r_{A}\right\} \Longleftrightarrow g_{i}(\mathbf{s})=1
$$

Furthermore, from Proposition 30c, we know that $\tilde{\mathbf{z}}_{C} \sim \tilde{d} \cdot \tilde{\mathbf{s}}(1)$ with $\tilde{d} \sim\left\|\tilde{\mathbf{z}}_{C}\right\|$. Thus,

$$
\mathbb{P}\left[\mathscr{E}_{i}\left[\tilde{\boldsymbol{z}}_{C}\right] \mid\left\|\tilde{\boldsymbol{z}}_{C}\right\| \leq r_{A}\right]=\mathbb{P}\left[\mathscr{E}_{i}[\tilde{d} \cdot \tilde{\mathbf{s}}(1)] \mid \tilde{d} \leq r_{A}\right]
$$


Next observe that, conditioned on $\tilde{d} \leq r_{A}$, the event $\mathscr{E}_{i}[\tilde{d} \cdot \tilde{\mathbf{s}}(1)]$ implies that

$$
f_{i}(\tilde{\mathbf{s}}(1))=1
$$

leading to

$$
\mathbb{P}\left[\mathscr{E}_{i}[\tilde{d} \tilde{\mathbf{s}}(1)] \mid \tilde{d} \leq r_{A}\right] \leq \mathbb{P}\left[f_{i}(\tilde{\mathbf{s}}(1))=1\right]
$$

Finally, noting that conditioned on $d=r_{A}, \tilde{\mathbf{u}}_{A} \sim \tilde{\mathbf{s}}(1)$. Thus,

$$
\begin{aligned}
& \mathbb{P}\left[\mathscr{E}_{i}\left[\tilde{\mathbf{u}}_{A}\right]\right]=\mathbb{P}_{\mathbf{s} \sim \tilde{\mathbf{s}}(1)}\left[\mathscr{E}_{i}[d \mathbf{s}] \mid d=r_{A}\right] \\
& =\mathbb{P}_{\mathbf{s} \sim \tilde{\mathbf{s}}(1)}\left[g_{i}(\mathbf{s})=1\right] \\
& \geq \mathbb{P}_{\mathbf{s} \sim \tilde{\mathbf{s}}(1)}\left[f_{i}(\mathbf{s})=1\right] \\
& \geq \mathbb{P}\left[\mathscr{E}_{i}\left[\tilde{\boldsymbol{z}}_{C}\right] \mid\left\|\tilde{\boldsymbol{z}}_{C}\right\| \leq r_{A}\right] . \quad \text { (from (4.23) and (4.24)) }
\end{aligned}
$$

(c) The proof is very similar to that of part (b) and is omitted.

Proposition 32. Let $\mathcal{C}$ be a code with codebook $\mathcal{B}$ and a minimum distance decoding function. Consider two noises $\tilde{\mathbf{z}}_{G} \sim N(\mathbf{0}, \sigma \cdot e)$ and $\tilde{\mathbf{z}}_{U}$ uniformly distributed on $\mathcal{S}_{n}\left(\sigma^{\prime} \sqrt{n}\right)$ with $\sigma^{\prime}<\sigma$. If $\mathbb{P}\left[\mathscr{E}_{i}\left[\tilde{\mathbf{z}}_{G}\right]\right] \leq \epsilon$, then

$$
\mathbb{P}\left[\mathscr{E}_{i}\left[\tilde{\mathbf{z}}_{U}\right]\right] \leq \frac{\epsilon}{1-\exp (-n \beta)}, \text { with } \beta=\frac{\sigma^{2}-\sigma^{\prime 2}-\log \left(1+\sigma^{2}-\sigma^{\prime 2}\right)}{2 \sigma^{2}}
$$

Proof.

Applying Proposition $31 \mathrm{c}$ with $\tilde{\mathbf{u}}_{A}=\tilde{\mathbf{z}}_{U}, r_{A}=\sigma^{\prime} \sqrt{n}$, and $\tilde{\mathbf{z}}_{G}=\tilde{\mathbf{z}}_{C}$, we obtain :

$$
\mathbb{P}\left[\mathscr{E}_{i}\left[\tilde{\mathbf{z}}_{U}\right]\right] \leq \mathbb{P}\left[\mathscr{E}_{i}\left[\tilde{\mathbf{z}}_{G}\right] \mid\left\|\tilde{\mathbf{z}}_{G}\right\|>\sigma^{\prime} \sqrt{n}\right]
$$


Furthermore,

$$
\begin{aligned}
\mathbb{P}\left[\left\|\tilde{\mathbf{z}}_{G}\right\|>\sigma^{\prime} \sqrt{n}\right] & =\mathbb{P}\left[\left\|\tilde{\mathbf{z}}_{G}\right\|^{2}>n \sigma^{\prime 2}\right] \\
& =\mathbb{P}\left[\left\|\tilde{\mathbf{z}}_{G}\right\|^{2}>n \sigma^{2}-\left(n \sigma^{2}-n \sigma^{\prime 2}\right)\right] \\
& =\mathbb{P}\left[\frac{1}{n}\left\|\tilde{\mathbf{z}}_{G}\right\|^{2}>\sigma^{2}-r\right]
\end{aligned}
$$

where $r=\sigma^{2}-\sigma^{\prime 2}$. Applying Proposition 30a, we have

$$
\mathbb{P}\left[\left\|\tilde{\mathbf{z}}_{G}\right\|>\sigma^{\prime} \sqrt{n}\right] \geq 1-\exp (-n \beta), \text { with } \beta=\frac{r-\log (1+r)}{2 \sigma^{2}} .
$$

Therefore, we have

$$
\begin{aligned}
\mathbb{P}\left[\mathscr{E}_{i}\left[\tilde{\mathbf{z}}_{G}\right]\right]= & \mathbb{P}\left[\mathscr{E}_{i}\left[\tilde{\mathbf{z}}_{G}\right] \mid\left\|\tilde{\mathbf{z}}_{G}\right\|>\sigma^{\prime} \sqrt{n}\right] \cdot \mathbb{P}\left[\left\|\tilde{\mathbf{z}}_{G}\right\|>\sigma^{\prime} \sqrt{n}\right] \\
& +\mathbb{P}\left[\mathscr{E}_{i}\left[\tilde{\mathbf{z}}_{G}\right] \mid\left\|\tilde{\mathbf{z}}_{G}\right\| \leq \sigma^{\prime} \sqrt{n}\right] \cdot \mathbb{P}\left[\left\|\tilde{\mathbf{z}}_{G}\right\| \leq \sigma^{\prime} \sqrt{n}\right] \\
\geq & \mathbb{P}\left[\mathscr{E}_{i}\left[\tilde{\mathbf{z}}_{G}\right] \mid\left\|\tilde{\mathbf{z}}_{G}\right\|>\sigma^{\prime} \sqrt{n}\right] \cdot \mathbb{P}\left[\left\|\tilde{\mathbf{z}}_{G}\right\|>\sigma^{\prime} \sqrt{n}\right] \\
\geq & \mathbb{P}\left[\mathscr{E}_{i}\left[\tilde{\mathbf{z}}_{U}\right]\right] \cdot \mathbb{P}\left[\left\|\tilde{\mathbf{z}}_{G}\right\|>\sigma^{\prime} \sqrt{n}\right] \quad(\text { from (4.25)) }
\end{aligned}
$$

which implies that

$$
\mathbb{P}\left[\mathscr{E}_{i}\left[\tilde{\mathbf{z}}_{U}\right]\right] \leq \frac{\mathbb{P}\left[\mathscr{E}_{i}\left[\tilde{\mathbf{z}}_{G}\right]\right]}{\mathbb{P}\left[\left\|\tilde{\mathbf{z}}_{G}\right\|>\sigma^{\prime} \sqrt{n}\right.} \leq \frac{\epsilon}{1-\exp (-n \beta)} \quad(\text { from }(4.26))
$$

We next examine certain properties of optimal solutions of (4.18). Let

$$
\mathcal{W}=\left\{\mathbf{w}_{t} \mid \mathbf{w}_{t}=\frac{1}{1+\nu} \mathbf{z}_{t}, \forall t=1, \ldots, T\right\}
$$

and $\tau(\mathbf{s})=\arg \operatorname{lex} \min _{t=1, \ldots, T}\left\|\mathbf{s}-\mathbf{w}_{t}\right\|$.

Proposition 33. Let $\epsilon>0$ be the constant used in (4.7), and $\gamma_{\epsilon}$ be as in (4.7). Let $\left\{r^{*}, \mathrm{x}_{i}, v_{i t}\right\}$ be an optimal solution of (4.18). For $\tilde{\mathbf{s}}$ uniformly distributed on $\mathcal{S}_{n}\left(\gamma_{\epsilon}\right)$, we have 
(a) $\mathbb{P}\left[\forall k \neq i:\left\|\mathbf{x}_{i}-\mathbf{x}_{k}+\tilde{\mathbf{s}}\right\| \geq \gamma_{\epsilon}\right] \geq \mathbb{P}\left[v_{i \tau(\tilde{\mathbf{s}})}=1\right]$,

(b) $\mathbb{P}\left[v_{i \tau(\tilde{\mathbf{s}})}=1\right] \geq 1-\epsilon$.

Proof.

(a) Constraint (4.11) implies that $\left\{\mathbf{x}_{i}\right\}_{i=1}^{2^{n R}}$ satisfy that, for the noise vector $\mathbf{z}_{t}$ 's in $\mathcal{Z}$ with $v_{i t}=1$,

$$
\begin{aligned}
\left\|\mathbf{x}_{i}-\mathbf{x}_{k}+\mathbf{z}_{t}\right\| \geq\left\|\mathbf{z}_{t}\right\|, \forall k \neq i, & \Longleftrightarrow\left\|\mathbf{x}_{i}-\mathbf{x}_{k}\right\|^{2} \geq 2\left\langle\mathbf{x}_{k}-\mathbf{x}_{i}, \mathbf{z}_{t}\right\rangle, \forall k \neq i \\
& \Longleftrightarrow\left\|\mathbf{x}_{i}-\mathbf{x}_{k}\right\|^{2} \geq 2(1+\nu)\left\langle\mathbf{x}_{k}-\mathbf{x}_{i}, \mathbf{w}_{t}\right\rangle, \forall k \neq i
\end{aligned}
$$

Since $\left\{\mathbf{z}_{1}, \mathbf{z}_{2}, \ldots, \mathbf{z}_{T}\right\}$ forms a Voronoi tessellation of $\mathcal{S}_{n}\left((1+\nu) \gamma_{\epsilon}\right)$, the set $\mathcal{W}$ also forms a Voronoi tessellation of $\mathcal{S}_{n}\left(\gamma_{\epsilon}\right)$. Therefore, letting $\mathbf{s}$ be any vector belonging to $\mathcal{S}^{n}$, from Proposition 29 applied to $\mathcal{A}=\mathcal{W}, N=T, \Lambda=\gamma_{\epsilon} / \sqrt{n}$, we obtain

$$
\left\|\mathbf{s}-\mathbf{w}_{\tau(\tilde{\mathbf{s}})}\right\| \leq \theta \sqrt{n}
$$

where $\tau(\mathbf{s})=\arg \min _{t=1, \ldots, T}\left\|\mathbf{s}-\mathrm{w}_{t}\right\|$, with

$$
\theta=\frac{\gamma_{\epsilon}}{\sqrt{n} T^{1 / n}}=\frac{\zeta \nu}{1+\nu}
$$

From (4.13) and (4.29), we have

$$
\left\|\mathbf{x}_{i}-\mathbf{x}_{k}\right\| \geq 2 \sqrt{n} \zeta=2 \theta \frac{1+\nu}{\nu} \sqrt{n}
$$


We now proceed to show that if $v_{i \tau(\mathbf{s})}=1$, then $\left\|\mathbf{x}_{i}-\mathbf{x}_{k}+\mathbf{s}\right\| \geq\|\mathbf{s}\|$. We have

$$
\begin{aligned}
2\left\langle\mathbf{x}_{k}-\mathbf{x}_{i}, \mathbf{s}\right\rangle & =2\left\langle\mathbf{x}_{k}-\mathbf{x}_{i},\left(\mathbf{s}-\mathbf{w}_{\tau(\mathbf{s})}\right)+\mathbf{w}_{\tau(\mathbf{s})}\right\rangle \\
& \leq 2\left\langle\mathbf{x}_{k}-\mathbf{x}_{i}, \mathbf{w}_{\tau(\mathbf{s})}\right\rangle+2 \cdot\left\|\mathbf{x}_{i}-\mathbf{x}_{k}\right\| \cdot\left\|\mathbf{s}-\mathbf{w}_{\tau(\mathbf{s})}\right\| \quad \text { (Cauchy-Schwartz) } \\
& \leq(1+\nu)^{-1}\left\|\mathbf{x}_{i}-\mathbf{x}_{k}\right\|^{2}+2 \cdot\left\|\mathbf{x}_{i}-\mathbf{x}_{k}\right\| \cdot \theta \sqrt{n} \quad \text { (from (4.27) and (4.28)) } \\
& =\left\|\mathbf{x}_{i}-\mathbf{x}_{k}\right\| \cdot\left\{(1+\nu)^{-1}\left\|\mathbf{x}_{i}-\mathbf{x}_{k}\right\|+2 \theta \sqrt{n}\right\} \\
& \leq\left\|\mathbf{x}_{i}-\mathbf{x}_{k}\right\| \cdot\left\{(1+\nu)^{-1}\left\|\mathbf{x}_{i}-\mathbf{x}_{k}\right\|+\frac{\nu}{1+\nu}\left\|\mathbf{x}_{i}-\mathbf{x}_{k}\right\|\right\} \quad \text { (from (4.30)) } \\
& =\left\|\mathbf{x}_{i}-\mathbf{x}_{k}\right\|^{2} .
\end{aligned}
$$

Therefore, if $v_{i \tau(\mathbf{s})}=1$, then

$$
2\left\langle\mathbf{x}_{k}-\mathbf{x}_{i}, \mathbf{s}\right\rangle \leq\left\|\mathbf{x}_{i}-\mathbf{x}_{k}\right\|^{2} \Longleftrightarrow\left\|\mathbf{x}_{i}-\mathbf{x}_{k}+\mathbf{s}\right\| \geq\|\mathbf{s}\|
$$

This implies that $\mathbb{P}\left[\forall k \neq i:\left\|\mathbf{x}_{i}-\mathbf{x}_{k}+\tilde{\mathbf{s}}\right\| \geq \gamma_{\epsilon}\right] \geq \mathbb{P}\left[v_{i r(\tilde{\mathbf{s}})}=1\right]$.

(b) For each $i \in \mathcal{B}$, we have

$$
\mathbb{P}\left[v_{i \tau(\tilde{\mathbf{s}})}=1\right]=\sum_{t=1}^{T} \mathbb{P}\left[v_{i \tau(\tilde{\mathbf{s}})}=1 \mid \tau(\tilde{\mathbf{s}})=t\right] \cdot \mathbb{P}[\tau(\tilde{\mathbf{s}})=t]
$$

We have

$$
\begin{aligned}
\mathbb{P}\left[v_{i \tau(\tilde{\mathbf{s}})}=1 \mid \tau(\tilde{\mathbf{s}})=t\right] & = \begin{cases}1, & \text { if } v_{i t}=1 \\
0, & \text { otherwise }\end{cases} \\
& =v_{i t}
\end{aligned}
$$

Moreover, since the set of vectors $\mathcal{W}$ forms a Voronoi tessellation of $\mathcal{S}_{n}\left(\gamma_{\epsilon}\right)$, the Voronoi regions of the points $\mathbf{w}_{t} \in \mathcal{W}$ are identical with the same area. Consequently, choosing $\tilde{\mathbf{s}}$ uniformly induces a uniform distribution for $\tau(\tilde{\mathbf{s}})$ on the elements of the set $\{1, \ldots, T\}$, that is,

$$
\mathbb{P}[\tau(\tilde{\mathbf{s}})=t]=\frac{1}{T}, \forall t=1, \ldots, T
$$


Substituting (4.32) and (4.33) in (4.31), we have

$$
\begin{aligned}
\mathbb{P}\left[v_{i \tau(\tilde{\mathbf{s}})}=1\right] & =\sum_{t=1}^{T} \mathbb{P}\left[v_{i \tau(\tilde{\mathbf{s}})}=1 \mid \tau(\tilde{\mathbf{s}})=t\right] \cdot \mathbb{P}[\tau(\tilde{\mathbf{s}})=t] \\
& =\frac{1}{T} \sum_{t=1}^{T} v_{i t} \geq \frac{1}{T} \cdot(1-\epsilon) T \quad(\text { from (4.14)) } \\
& =1-\epsilon .
\end{aligned}
$$

Recall that $\mathcal{R}_{n}[P, \sigma, \epsilon]$ is the set of all achievable rates on a single user Gaussian channel with power $P$, standard deviation of the noise $\sigma$ and maximum decoding error probability of $\epsilon$. We are now in a position to present our main result.

Theorem 34. Let $r^{*},\left\{\mathbf{x}_{i}\right\}_{i=1}^{2^{n R}}$, be an optimal solution of (4.18),

(a) If $r^{*}=1$, then $R \in \mathcal{R}_{n}[P, \sigma, 2 \epsilon]$, that is the rate $R$ is achievable using the codebook $\mathcal{B}=\left\{\mathbf{x}_{i}\right\}_{i=1}^{2^{n R}}$ and the minimum distance decoding function, achieving a maximum decoding error probability of $2 \epsilon$.

(b) If $r^{*} \geq 2$, then $R \notin \mathcal{R}_{n}\left[P,(1+3 \nu) \sigma, \epsilon^{\prime}\right]$, where $\epsilon^{\prime}=(1-\delta(\nu, n)) \epsilon$

$$
\begin{aligned}
\delta(\nu, n) & =\exp \left(-n \cdot \frac{r-\log (1+r)}{2(1+3 \nu)^{2} \sigma^{2}}\right), \text { with } r=\sigma^{2}\left((1+3 \nu)^{2}-(1+2 \nu)^{2}\right) \\
& \rightarrow 0, \text { as } n \rightarrow \infty
\end{aligned}
$$

Proof.

(a) We consider a Gaussian channel with noise $\tilde{\mathbf{z}}_{G} \sim N(\mathbf{0}, \sigma \boldsymbol{e})$. From Proposition 2, we have

$$
\mathbb{P}\left[\overline{\mathbf{z}}_{G} \notin \mathcal{U}_{\epsilon}^{\text {Normal }}\right] \leq \epsilon
$$

where $\mathcal{U}_{\epsilon}^{\text {Normal }}=\left\{\mathbf{s} \in \mathbb{R}^{n} \mid\|\mathbf{s}\| \leq \gamma_{\epsilon}\right\}$, with $\gamma_{\epsilon}=\sqrt{n \sigma^{2}+\Gamma_{\epsilon}}$. We next consider the 
probability of error when a codeword $i$ is sent on the channel :

$$
\begin{aligned}
\mathbb{P}\left[\mathscr{E}_{i}\left[\tilde{\mathbf{z}}_{G}\right]\right]= & \mathbb{P}\left[\exists k \neq i:\left\|\mathbf{x}_{i}-\mathbf{x}_{k}+\tilde{\mathbf{z}}_{G}\right\| \leq\left\|\tilde{\mathbf{z}}_{G}\right\|\right] \\
= & \int_{0}^{\gamma_{\epsilon}} \mathbb{P}\left[\exists k \neq i:\left\|\mathbf{x}_{i}-\mathbf{x}_{k}+\tilde{\mathbf{z}}_{G}\right\| \leq\left\|\tilde{\mathbf{z}}_{G}\right\|,\left\|\tilde{\mathbf{z}}_{G}\right\| \in(c-\mathrm{d} c, c]\right] \mathrm{d} c \\
& +\int_{\gamma_{\epsilon}}^{\infty} \mathbb{P}\left[\exists k \neq i:\left\|\mathbf{x}_{i}-\mathbf{x}_{k}+\tilde{\mathbf{z}}_{G}\right\| \leq\left\|\tilde{\mathbf{z}}_{G}\right\|,\left\|\tilde{\mathbf{z}}_{G}\right\| \in(c-\mathrm{d} c, c]\right] \mathrm{d} c
\end{aligned}
$$

We bound the second term as follows :

$$
\begin{gathered}
\int_{\gamma_{\epsilon}}^{\infty} \mathbb{P}\left[\exists k \neq i:\left\|\mathbf{x}_{i}-\mathbf{x}_{k}+\tilde{\mathbf{z}}_{G}\right\| \leq\left\|\tilde{\mathbf{z}}_{G}\right\|,\left\|\tilde{\mathbf{z}}_{G}\right\| \in(c-\mathrm{d} c, c]\right] \mathrm{d} c \\
\quad \leq \int_{\gamma_{\epsilon}}^{\infty} \mathbb{P}\left[\left\|\tilde{\mathbf{z}}_{G}\right\| \in(c-\mathrm{d} c, c]\right] \mathrm{d} c=\mathbb{P}\left[\tilde{\mathbf{z}}_{G} \notin \mathcal{U}_{\epsilon}^{\text {Normal }}\right] \leq \epsilon .
\end{gathered}
$$

We bound the first term as follows:

$$
\begin{aligned}
& \int_{0}^{\gamma_{e}} \mathbb{P}\left[\exists k \neq i:\left\|\mathbf{x}_{i}-\mathbf{x}_{k}+\tilde{\mathbf{z}}_{G}\right\| \leq\left\|\tilde{\mathbf{z}}_{G}\right\|,\left\|\tilde{\mathbf{z}}_{G}\right\| \in(c-\mathrm{d} c, c]\right] \mathrm{d} c \\
& =\int_{0}^{\gamma_{\epsilon}} \mathbb{P}\left[\exists k \neq i:\left\|\mathbf{x}_{i}-\mathbf{x}_{k}+\tilde{\mathbf{z}}_{G}\right\| \leq\left\|\tilde{\mathbf{z}}_{G}\right\| \mid\left\|\tilde{\mathbf{z}}_{G}\right\| \in(c-\mathrm{d} c, c]\right] \\
& \cdot \mathbb{P}\left[\left\|\tilde{\mathbf{z}}_{G}\right\| \in(c-\mathrm{d} c, c]\right] \mathrm{d} c \\
& \leq \int_{0}^{\gamma_{\epsilon}} \mathbb{P}\left[\exists k \neq i:\left\|\mathbf{x}_{i}-\mathbf{x}_{k}+\tilde{\mathbf{s}}(c)\right\| \leq c\right] \mathbb{P}\left[\left\|\tilde{\mathbf{z}}_{G}\right\| \in(c-\mathrm{d} c, c]\right] \mathrm{d} c \quad \text { (Prop. 31(b)) } \\
& \leq \int_{0}^{\gamma_{\epsilon}} \mathbb{P}\left[\exists k \neq i:\left\|\mathbf{x}_{i}-\mathbf{x}_{k}+\tilde{\mathbf{s}}\left(\gamma_{\epsilon}\right)\right\| \leq \gamma_{\epsilon}\right] \mathbb{P}\left[\left\|\tilde{\mathbf{z}}_{G}\right\| \in(c-\mathrm{d} c, c]\right] \mathrm{d} c \quad \text { (Prop. 31(a)) } \\
& \leq \int_{0}^{\gamma_{\epsilon}}\left(1-\mathbb{P}\left[v_{i \tau_{\tilde{\mathbf{B}}\left(\gamma_{\epsilon}\right)}}=1\right]\right) \mathbb{P}\left[\left\|\tilde{\mathbf{z}}_{G}\right\| \in(c-\mathrm{d} c, c]\right] \mathrm{d} c \\
& \leq \int_{0}^{\gamma_{\epsilon}}(1-(1-\epsilon)) \mathbb{P}\left[\left\|\tilde{\mathbf{z}}_{G}\right\| \in(c-\mathrm{d} c, c]\right] \mathrm{d} c \\
& \leq \epsilon \int_{0}^{\gamma_{\epsilon}} \mathbb{P}\left[\left\|\tilde{\mathbf{z}}_{G}\right\| \in(c-\mathrm{d} c, c]\right] \mathrm{d} c \leq \epsilon
\end{aligned}
$$

From (4.35) and (4.36), we have $\mathbb{P}\left[\mathscr{E}_{i}\left[\tilde{\boldsymbol{z}}_{G}\right]\right] \leq 2 \epsilon$, and the theorem follows. 
(b) To prove this part, we choose a rate $R \in \mathcal{R}_{n}\left(P,(1+3 \nu) \sigma, \epsilon^{\prime}\right)$, and then show that there exists a code that is feasible to Constraints (4.9)-(4.15). Since $R \in$ $\mathcal{R}_{n}\left(P,(1+3 \nu) \sigma, \epsilon^{\prime}\right)$, there exists a code $\mathcal{C}$ that has an error-probability of $\epsilon^{\prime}$ on the channel with Gaussian noise $\tilde{\mathbf{z}}_{G} \sim N(\mathbf{0},(1+3 \nu) \sigma \cdot \boldsymbol{e})$, using codewords $\left\{\mathbf{x}_{i}\right\}_{i=1}^{2^{n R}}$, satisfying

$$
\mathbb{P}\left[\mathscr{E}_{i}\left[\tilde{z}_{G}\right]\right]=\mathbb{P}\left[\left\{\exists k \neq i:\left\|\mathbf{x}_{i}-\mathbf{x}_{k}+\tilde{\mathbf{z}}_{G}\right\| \leq\left\|\tilde{\mathbf{z}}_{G}\right\|\right\}\right] \leq \epsilon^{\prime}
$$

We first show that the codewords $\left\{\mathbf{x}_{i}\right\}_{i=1}^{2^{n R}}$ satisfy Eq.(4.13). Consider the probability of incorrectly decoding $\mathbf{x}_{i}$ as $\mathbf{x}_{k}$ on this channel. We have

$$
\begin{aligned}
\mathbb{P}\left[\mathbf{x}_{i} \text { decoded as } \mathbf{x}_{k}\right] & \geq \mathbb{P}\left[\left\|\mathbf{x}_{i}+\tilde{\mathbf{z}}_{G}-\mathbf{x}_{k}\right\| \leq\left\|\tilde{\mathbf{z}}_{\mathrm{G}}\right\|\right] \\
& =\mathbb{P}\left[2\left\langle\mathbf{x}_{k}-\mathbf{x}_{i}, \tilde{\mathbf{z}}_{G}\right\rangle \geq\left\|\mathbf{x}_{i}-\mathbf{x}_{k}\right\|^{2}\right] \\
& =\mathbb{P}\left[\frac{\left\langle\mathbf{x}_{k}-\mathbf{x}_{i}, \tilde{\mathbf{z}}_{G}\right\rangle}{(1+3 \nu) \sigma\left\|\mathbf{x}_{i}-\mathbf{x}_{k}\right\|} \geq \frac{\left\|\mathbf{x}_{i}-\mathbf{x}_{k}\right\|}{2(1+3 \nu) \sigma}\right] \\
& =1-\Phi\left(\frac{\left\|\mathbf{x}_{i}-\mathbf{x}_{k}\right\|}{2(1+3 \nu) \sigma}\right) .
\end{aligned}
$$

Since we know that $\mathbb{P}\left[\mathbf{x}_{i}\right.$ decoded as $\left.\mathbf{x}_{k}\right] \leq \mathbb{P}\left[\mathscr{E}_{i}\left[\tilde{\boldsymbol{z}}_{G}\right]\right] \leq \epsilon^{\prime}$, we have

$$
\begin{aligned}
& \epsilon^{\prime} \geq 1-\Phi\left(\frac{\left\|\mathbf{x}_{i}-\mathbf{x}_{k}\right\|}{2(1+3 \nu) \sigma}\right) \\
\Longrightarrow & \left\|\mathbf{x}_{i}-\mathbf{x}_{k}\right\| \geq 2(1+3 \nu) \sigma \Phi^{-1}\left(1-\epsilon^{\prime}\right)=2(1+3 \nu) \zeta \sqrt{n},
\end{aligned}
$$

which implies that (4.13) is satisfied.

We next show that the codewords $\left\{\mathbf{x}_{i}\right\}_{i=1}^{2^{n R}}$ satisfy Eqs.(4.11) and (4.14). Let $\tilde{\mathbf{z}}_{U}$ be uniformly distributed in $\mathcal{S}_{n}\left(\sigma_{1} \sqrt{n}\right)$, where

$$
\sigma_{1}=(1+2 \nu) \sigma<(1+3 \nu) \sigma
$$

and let $\tilde{\mathbf{z}}_{D}$ be a discrete uniform distribution given by

$$
\mathbb{P}\left[\tilde{\mathbf{z}}_{D}=\mathbf{z}_{t}\right]=\frac{1}{T}, \forall t=1, \ldots, T .
$$


Consider a $\mathbf{z}_{t}$, and letting $\mathbf{u}_{t}=\sigma_{1} \mathbf{z}_{t} /(1+\nu) \sigma$, we have $\left\|\mathbf{u}_{t}\right\|=\sigma_{1} \sqrt{n}$. Let $\mathbf{s} \in$ $\mathcal{S}_{n}\left(\sigma_{1} \sqrt{n}\right)$ such that $\mathbf{s}$ is in the Voronoi region $\mathcal{V}\left(\mathbf{u}_{t}\right)$ of $\mathbf{u}_{t}$, that is, $\mathbf{s} \in \mathcal{V}\left(\mathbf{u}_{t}\right)$. Applying Proposition 29 to $\mathcal{A}=\left\{\mathbf{u}_{1}, \ldots, \mathbf{u}_{T}\right\}, N=T, \Lambda=\sigma_{1}$, we obtain

$$
\left\|\mathbf{s}-\mathbf{u}_{t}\right\| \leq \theta^{\prime} \sqrt{n}
$$

where

$$
\begin{aligned}
\theta^{\prime} & =\frac{\sigma_{1}}{T^{1 / n}}=\frac{\sigma_{1} \sqrt{n}}{\gamma_{\epsilon}} \cdot \frac{\gamma_{\epsilon}}{\sqrt{n} T^{1 / n}}=\frac{\sigma_{1} \sqrt{n}}{\gamma_{\epsilon}} \cdot \zeta \cdot \frac{\nu}{1+\nu} \quad(\text { from }(4.29)) \\
& \leq \frac{\sigma_{1} \sqrt{n}}{\gamma_{\epsilon}} \cdot \frac{1}{1+2 \nu} \cdot \frac{1}{2 \sqrt{n}} \cdot\left\|\mathbf{x}_{i}-\mathbf{x}_{k}\right\| \cdot \frac{\nu}{1+\nu} \quad(\text { from }(4.37)) \\
& =\frac{(1+2 \nu) \sigma \sqrt{n}}{\gamma_{\epsilon}} \cdot \frac{1}{1+2 \nu} \cdot \frac{\nu}{1+\nu} \cdot \frac{1}{2 \sqrt{n}} \cdot\left\|\mathbf{x}_{i}-\mathbf{x}_{k}\right\| \leq \frac{\nu}{1+\nu} \cdot \frac{1}{2 \sqrt{n}} \cdot\left\|\mathbf{x}_{i}-\mathbf{x}_{k}\right\| .
\end{aligned}
$$

Finally, if $\left\|\mathbf{x}_{i}-\mathbf{x}_{k}+\mathbf{z}_{t}\right\| \leq\left\|\mathbf{z}_{t}\right\|$, then

$$
\begin{aligned}
2\left\langle\mathbf{x}_{k}-\mathbf{x}_{i}, \mathbf{s}\right\rangle & =2\left\langle\mathbf{x}_{k}-\mathbf{x}_{i},\left(\mathbf{s}-\mathbf{u}_{t}\right)+\mathbf{u}_{t}\right\rangle \\
& \geq 2\left\langle\mathbf{x}_{k}-\mathbf{x}_{i}, \mathbf{u}_{t}\right\rangle-2 \cdot\left\|\mathbf{x}_{i}-\mathbf{x}_{k}\right\| \cdot\left\|\mathbf{s}-\mathbf{u}_{t}\right\| \quad \text { (Cauchy-Schwartz) } \\
& \geq \frac{1+2 \nu}{1+\nu}\left\|\mathbf{x}_{i}-\mathbf{x}_{k}\right\|^{2}-2 \cdot\left\|\mathbf{x}_{i}-\mathbf{x}_{k}\right\| \cdot \theta^{\prime} \sqrt{n} \quad \text { (from (4.38)) } \\
& =\left\|\mathbf{x}_{i}-\mathbf{x}_{k}\right\| \cdot\left\{\frac{1+2 \nu}{1+\nu}\left\|\mathbf{x}_{i}-\mathbf{x}_{k}\right\|-2 \theta^{\prime} \sqrt{n}\right\} \\
& \geq\left\|\mathbf{x}_{i}-\mathbf{x}_{k}\right\| \cdot\left\{\frac{1+2 \nu}{1+\nu}\left\|\mathbf{x}_{i}-\mathbf{x}_{k}\right\|-\frac{\nu}{1+\nu} \cdot\left\|\mathbf{x}_{i}-\mathbf{x}_{k}\right\|\right\} \quad \text { (from (4.39)) } \\
& =\left\|\mathbf{x}_{i}-\mathbf{x}_{k}\right\|^{2}
\end{aligned}
$$

which is equivalent to $\left\|\mathbf{x}_{i}-\mathbf{x}_{k}+\mathbf{s}\right\| \leq\|\mathbf{s}\| \forall \mathbf{s} \in \mathcal{V}\left(\mathbf{u}_{t}\right)$. Therefore,

$$
\text { if }\left\|\mathbf{x}_{i}-\mathbf{x}_{k}+\mathbf{z}_{t}\right\| \leq\left\|\mathbf{z}_{t}\right\| \text {, then }\left\|\mathbf{x}_{i}-\mathbf{x}_{k}+\mathbf{s}\right\| \leq\|\mathbf{s}\| \forall \mathbf{s} \in \mathcal{V}\left(\mathbf{u}_{t}\right)
$$


, then Furthermore,

$$
\mathbb{P}\left[\mathscr{E}_{i}\left[\tilde{\boldsymbol{z}}_{U}\right]\right]=\sum_{t=1}^{T} \mathbb{P}\left[\mathscr{E}_{i}\left[\tilde{\boldsymbol{z}}_{U}\right] \mid \tilde{\boldsymbol{z}}_{U} \in \mathcal{V}\left(\mathbf{u}_{t}\right)\right], \mathbb{P}\left[\tilde{\boldsymbol{z}}_{U} \in \mathcal{V}\left(\mathbf{u}_{t}\right)\right]
$$

Since the set of vectors $\left\{\mathbf{z}_{t}\right\}$ form a Voronoi tessellation, the vectors $\left\{\mathbf{u}_{t}\right\}$ also form a Voronoi tessellation on $\mathcal{S}^{n}\left(\sigma_{1} \sqrt{n}\right)$. Therefore, the Voronoi regions of the points $\mathbf{u}_{t}$ are identical with the same area. Consequently,

$$
\mathbb{P}\left[\tilde{\boldsymbol{z}}_{U} \in \mathcal{V}\left(\mathbf{u}_{t}\right)\right]=\frac{1}{T}, \forall t=1, \ldots, T
$$

Moreover, from (4.40), we have

$$
\mathbb{P}\left[\mathscr{E}_{i}\left[\tilde{\boldsymbol{z}}_{U}\right] \mid \tilde{\boldsymbol{z}}_{U} \in \mathcal{V}\left(\mathbf{u}_{t}\right)\right] \geq 1\left\{\left\|\mathbf{x}_{i}-\mathbf{x}_{k}+\mathbf{z}_{t}\right\| \leq\left\|\mathbf{z}_{t}\right\|\right\}
$$

Substituting (4.42) and (4.43) in (4.41), we have

$$
\mathbb{P}\left[\mathscr{E}_{i}\left[\tilde{z}_{U}\right]\right] \geq \frac{1}{T} \sum_{t=1}^{T} 1\left\{\left\|\mathbf{x}_{i}-\mathbf{x}_{k}+\mathbf{z}_{t}\right\| \leq\left\|\mathbf{z}_{t}\right\|\right\}
$$

From Proposition 32 we have,

$$
\mathbb{P}\left[\mathscr{E}_{i}\left[\tilde{\boldsymbol{z}}_{U}\right]\right] \leq \frac{\mathbb{P}\left[\mathscr{E}_{i}\left[\tilde{\boldsymbol{z}}_{G}\right]\right]}{1-\delta(\nu, n)} \leq \frac{\epsilon^{\prime}}{1-\delta(\nu, n)}=\epsilon
$$

Therefore, from (4.44), we have

$$
\frac{1}{T} \sum_{t=1}^{T} \mathbf{1}\left\{\left\|\mathbf{x}_{i}-\mathbf{x}_{k}+\mathbf{z}_{t}\right\| \leq\left\|\mathbf{z}_{t}\right\|\right\} \leq \mathbb{P}\left[\mathscr{E}_{i}\left[\tilde{z}_{U}\right]\right] \leq \epsilon,
$$

which implies that the codewords satisfy constraints (4.14).

Recall that the asymptotic capacity region $\mathcal{R}[P, \sigma]$ is defined as

$$
\mathcal{R}[P, \sigma]=\lim _{n \rightarrow \infty} \mathcal{R}_{n}\left[P, \sigma, \epsilon_{n}\right], \text { where } \epsilon_{n} \rightarrow 0, \text { as } n \rightarrow \infty
$$


Theorem 34 provides an exact characterization of the asymptotic capacity region while simultaneously constructing the matching code. In his characterization of the capacity region, Shannon [1948a] used the probabilistic method to show the existence of matching codes. In the last six decades, there has been substantial research in constructing codes that are optimal for different regimes of $P, \sigma$ (see Verdu and McLaughlin [1998] for a comprehensive review).

The codes that we construct by solving (4.18) provides a systematic method for constructing optimal codes for all parameter values $P, \sigma$. Moreover, Theorem 34 provides a characterization of the capacity region and optimal coding for the finite $n$ case. While in this case, our characterization is not tight, we provide explicit bounds of the error probability for given $n$ and for error probability $\epsilon$.

On the negative side, our characterization of the capacity region is algorithmic in nature, while Shannon characterized the capacity region in closed form. In addition, computationally we need to solve large scale NP-hard problems to construct the optimal code. However, we report computational evidence in Section 6 that suggests we can construct optimal codes up to $M=100,000$ and $n=50-60$. Most importantly, as we show in the next section, our algorithmic approach generalizes to multi-user channels with interference.

\subsection{Capacity Region of a Gaussian Interference Channel}

A two-user interference channel is a communication medium in which each user intends to transmit messages taken from their corresponding message books $\mathcal{B}^{1}=$ $\left\{1,2, \ldots, M_{1}\right\}$ and $\mathcal{B}^{2}=\left\{1,2, \ldots, M_{2}\right\}$. User 1 selects message $m^{1}=i \in \mathcal{B}^{1}$ and transmits $\mathbf{x}_{i}^{1}$ over the channel, while satisfying the power constraint

$$
\left\|\mathbf{x}_{i}^{1}\right\|^{2} \leq P_{1}
$$


User 2 chooses message $m^{2}=k \in \mathcal{B}^{2}$ and transmits $\mathbf{x}_{k}^{2}$ that satisfies

$$
\left\|\mathrm{x}_{k}^{2}\right\|^{2} \leq P_{2}
$$

The channel introduces noise terms $\tilde{\mathbf{z}}^{1}$ and $\tilde{\mathbf{z}}^{2}$, and the transmitted messages $\mathbf{x}_{i}^{1}$ and $\mathbf{x}_{k}^{2}$ interfere to give rise to codewords $\mathbf{y}^{1}$ and $\mathbf{y}^{2}$ given by

$$
\begin{aligned}
& \mathbf{y}^{1}=\mathbf{x}_{i}^{1}+h_{12} \mathbf{x}_{k}^{2}+\tilde{\mathbf{z}}^{1} \\
& \mathbf{y}^{2}=\mathbf{x}_{k}^{2}+h_{21} \mathbf{x}_{i}^{1}+\tilde{\mathbf{z}}^{2}
\end{aligned}
$$

which are received by the users. The noise terms $\tilde{\mathbf{z}}^{1}$ and $\tilde{\mathbf{z}}^{2}$ are assumed to be Gaussian noise, and the interference parameters $h_{12}$ and $h_{21}$ are assumed to be real numbers. We let $m^{1}=i$ and $m^{2}=k$ to denote that Users 1 and 2 transmitted messages $i \in \mathcal{B}^{1}$ and $k \in \mathcal{B}^{2}$ on the channel, respectively.

The channel coding problem for a two-user Gaussian interference channel refers to the problem of constructing a code with inputs:

(a) The length $n$ of the codewords;

(b) The rates $R_{1}, R_{2}$ of the code;

(c) The common standard deviation $\sigma$ of the normally distributed noises $\tilde{\mathbf{z}}^{1}$ and $\tilde{\mathbf{z}}^{2}$;

(d) The power constraints $P_{1}, P_{2}$ of the users;

(e) The average probability of error $\epsilon>0$ (see Eqs.(4.46) and (4.47)) the users tolerate.

The outputs of $\mathcal{C}^{\mathrm{IC}}\left[n, R_{1}, R_{2}, P_{1}, P_{2}, \sigma, \epsilon\right]$ are:

(a) The codebooks $\mathcal{B}^{1}=\left\{\mathbf{x}_{i}^{1}\right\}_{i=1}^{2^{n R_{1}}}, \mathcal{B}^{2}=\left\{\mathbf{x}_{k}^{2}\right\}_{k=1}^{2^{n R_{2}}}$;

(b) The decoding functions $h^{1}: \mathbb{R}^{n} \rightarrow \mathcal{B}^{1}, h^{2}: \mathbb{R}^{n} \rightarrow \mathcal{B}^{2}$ that map each received codeword $\mathbf{y}^{1}, \mathbf{y}^{2}$ to one of the codewords in $\mathcal{B}^{1}$ and $\mathcal{B}^{2}$, respectively, so that the 
average probability of error satisfies

$$
\begin{gathered}
\frac{1}{2^{n R_{1}}} \sum_{i=1}^{2^{n R_{1}}} \mathbb{P}\left[h^{1}\left(\mathbf{y}^{1}\right) \neq i \mid m^{1}=i\right] \leq \epsilon \\
\frac{1}{2^{n R_{2}}} \sum_{k=1}^{2^{n R_{2}}} \mathbb{P}\left[h^{2}\left(\mathbf{y}^{2}\right) \neq k \mid m^{2}=k\right] \leq \epsilon .
\end{gathered}
$$

Given these definitions, the capacity region $\mathcal{R}_{n}^{\mathrm{IC}}\left[P_{1}, P_{2}, \sigma, \epsilon\right]$ is defined as the set of all rate pairs $\left(R_{1}, R_{2}\right)$ such that there exists a code $\mathcal{C}^{\mathrm{IC}}\left[n, R_{1}, R_{2}, P_{1}, P_{2}, \sigma, \epsilon\right]$.

\subsubsection{Capacity Characterization and Optimal Coding}

In this section, we present the algorithm which we use to characterize the capacity region while simultaneously constructing the optimal code. The algorithm consists of two parts: (a) the Encoding Algorithm (Algorithm 2), and (b) the Decoding Algorithm (Algorithm 3). A key ingredient in our construction of this algorithm is the form of the decoder (4.48) that we propose. In this proposal, User 1(2) after receiving $\mathbf{y}^{1}\left(\mathbf{y}^{2}\right)$, selects $i_{1}^{*}\left(i_{2}^{*}\right)$ by solving the minimization problems:

$$
\begin{aligned}
& i_{1}^{*}=\arg \min _{i \in\left\{1, \ldots, M_{1}\right\}}\left[\min _{k \in\left\{1, \ldots, M_{2}\right\}}\left\|\mathbf{y}^{1}-\left(\mathbf{x}_{i}^{1}+h_{12} \mathbf{x}_{k}^{2}\right)\right\|\right], \\
& i_{2}^{*}=\arg \min _{k \in\left\{1, \ldots, M_{2}\right\}}\left[\min _{i \in\left\{1, \ldots, M_{1}\right\}}\left\|\mathbf{y}^{1}-\left(\mathbf{x}_{i}^{2}+h_{21} \mathbf{x}_{k}^{1}\right)\right\|\right] .
\end{aligned}
$$

In Theorem 40, we show that it suffices to restrict to this decoder in order to construct capacity characterizing codewords. Based on this decoder, we next present the Encoding Algorithm which involves checking the feasibility of Problem (4.49-4.65). The inputs to the encoding algorithm are $\left(n, R_{1}, R_{2}, P_{1}, P_{2}, \epsilon, \nu\right)$, where the parameter $\nu>0$ controls how close the algorithm characterizes the capacity region. Using these inputs, we first calculate the "derivative" quantities: $\Gamma_{\epsilon}, T, M_{0}$, and the set of vectors $\mathcal{Z}=\left\{\mathbf{z}_{1}, \mathbf{z}_{2}, \ldots, \mathbf{z}_{T}\right\}$ as defined in (4.7)-(4.8). The feasibility problem involves the following decision variables:

1. Variables $\left\{\mathbf{x}_{i}^{1}\right\}_{i=1}^{2^{n R_{1}}},\left\{\mathbf{x}_{k}^{2}\right\}_{k=1}^{2^{n R_{2}}}$ that represent the codewords for Users 1 and 2 , 
respectively;

2. Binary variables $v_{i}^{1}, v_{k}^{2}, v_{i k}^{1}, v_{i k}^{2}$ that are used to identify the subset of codewords which will be decoded correctly with probability at least $1-\epsilon^{\frac{1}{4}}$. In particular:

(a) $v_{i}^{1}=1$, if for codeword $i$ of User 1, the average probability of error is at most $\epsilon^{\frac{1}{4}}$, that is,

$$
\frac{1}{2^{n R_{2}}} \sum_{k=1}^{2^{n R_{2}}} \mathbb{P}\left[g^{1}\left(\mathrm{y}^{1}\right) \neq i \mid m^{1}=i, m^{2}=k\right] \leq \epsilon^{\frac{1}{4}}
$$

and $v_{i}^{1}=0$, otherwise, where $g^{1}(\cdot)$ is defined in (4.66). The binary variables $v_{k}^{2}, \forall k=1, \ldots, 2^{n R_{2}}$ are defined in a similar fashion.

(b) $v_{i k}^{1}=1$, if whenever $m^{1}=i$ and $m^{2}=k$, User 1 is able to correctly decode with probability at least $1-\epsilon^{\frac{1}{4}}$, that is,

$$
\mathbb{P}\left[g^{1}\left(\mathbf{y}^{1}\right) \neq i \mid m^{1}=i, m^{2}=k\right] \leq \epsilon^{\frac{1}{4}}
$$

and $v_{i k}^{1}=0$ otherwise. The variables $v_{i k}^{2}, \forall i, k$ are defined in a similar fashion.

3. Binary variables $\left\{v_{i k t}^{1}, v_{i k t}^{2}\right\}_{t=1}^{T}$ are axillary variables which allow us to constrain the probability of incorrect decoding for codewords identified by $v_{i}^{1}, v_{k}^{2}, v_{i k}^{1}, v_{i k}^{2}$. In particular, for codewords $i, i^{\prime}, k, k^{\prime}$ with $v_{i k}^{1}=1$ and $v_{i^{\prime} k^{\prime}}^{1}=1$,

$$
v_{i k t}^{1}= \begin{cases}1, & \text { if }\left\|\left(\mathbf{x}_{i}^{1}+h_{12} \mathbf{x}_{k}^{2}\right)-\left(\mathbf{x}_{i^{\prime}}^{1}+h_{12} \mathbf{x}_{k^{\prime}}^{2}\right)+\mathbf{z}_{t}\right\| \geq\left\|\mathbf{z}_{t}\right\| \\ 0, & \text { otherwise. }\end{cases}
$$

We next present Algorithm 3 for decoding, which is parametrized by the quantities $v_{i}^{1}, v_{k}^{2}, v_{i k}^{1}, v_{i k}^{2}$ that are computed by Algorithm 2.

In Algorithm 2, constraints (4.49) and (4.50) impose power constraints on the codewords. Constraints (4.51)-(4.57) implement the decoding constraints for User 1. 
Algorithm 2 Encoding algorithm for two-user interference channel.

Input: $n, R_{1}, R_{2}, \sigma, P_{1}, P_{2}, \epsilon, \nu$.

Output: Codewords $\mathbf{x}_{i}^{1}, \mathbf{x}_{k}^{2}$, and binary variables $v_{i}^{1}, v_{k}^{2}, v_{i k}^{1}, v_{i k}^{2},\left\{v_{i k t}^{1}, v_{i k t}^{2}\right\}_{t=1}^{T}$. Algorithm:

1. Check the feasibility of the constraints

$$
\begin{aligned}
& \left\|\mathbf{x}_{i}^{1}\right\|^{2} \leq n P_{1} \quad \forall i \\
& \left\|\mathrm{x}_{k}^{2}\right\|^{2} \leq n P_{2} \quad \forall k, \\
& \left\|\mathbf{x}_{i}^{1}-\mathbf{x}_{i^{\prime}}^{1}+h_{12}\left(\mathbf{x}_{k}^{2}-\mathbf{x}_{k^{\prime}}^{2}\right)+\mathbf{z}_{t}\right\| \\
& +\left(2-v_{i k t}^{1}-v_{i^{\prime} k^{\prime}}^{1}\right) M_{0} \geq\left\|\mathbf{z}_{t}\right\|, \quad \forall i, i^{\prime}, k, k^{\prime}, t \\
& \left\|\mathbf{x}_{i}^{1}-\mathbf{x}_{i^{\prime}}^{1}+h_{12}\left(\mathbf{x}_{k}^{2}-\mathbf{x}_{k^{\prime}}^{2}\right)\right\| \geq 2 \sigma \Phi^{-1}\left(1-\epsilon^{\frac{1}{4}}\right), \quad \forall i, i^{\prime}, k, k^{\prime}, \\
& v_{i k t}^{1} \leq v_{i k}^{1} \quad \forall i, i^{\prime}, k, k^{\prime}, t, \\
& \sum_{t=1}^{T} v_{i k t}^{1} \geq\left(1-\epsilon^{\frac{1}{4}}\right) T \cdot v_{i k}^{1} \quad \forall i, i^{\prime}, k, k^{\prime}, t \\
& v_{i k}^{1} \leq v_{i}^{1} \quad \forall i, k, \\
& \sum_{k=1}^{2^{n R_{2}}} v_{i k}^{1} \geq\left(1-\epsilon^{\frac{1}{4}}\right) 2^{n R_{2}} \cdot v_{i}^{1} \quad \forall i \\
& \sum_{i=1}^{2^{n R_{1}}} v_{i}^{1} \geq\left(1-\epsilon^{\frac{1}{4}}\right) \cdot 2^{n R_{1}} \\
& \left\|\mathbf{x}_{k}^{2}-\mathbf{x}_{k^{\prime}}^{2}+h_{21}\left(\mathbf{x}_{i}^{1}-\mathbf{x}_{i^{\prime}}^{1}\right)+\mathbf{z}_{t}\right\| \\
& +\left(2-v_{i k t}^{2}-v_{i^{\prime} k^{\prime}}^{2}\right) M_{0} \geq\left\|\mathbf{z}_{t}\right\|, \quad \forall i, i^{\prime}, k, k^{\prime}, t, \\
& \left\|\mathrm{x}_{k}^{2}-\mathrm{x}_{k^{\prime}}^{2}+h_{21}\left(\mathbf{x}_{i}^{1}-\mathbf{x}_{i^{\prime}}^{1}\right)\right\| \geq 2 \sigma \Phi^{-1}\left(1-\epsilon^{\frac{1}{4}}\right), \quad \forall i, i^{\prime}, k, k^{\prime}, \\
& v_{i k t}^{2} \leq v_{i k}^{2} \quad \forall i, i^{\prime}, k, k^{\prime}, t \\
& \sum_{t=1}^{T} v_{i k t}^{2} \geq\left(1-\epsilon^{\frac{1}{4}}\right) T \cdot v_{i k}^{2}, \quad \forall i, i^{\prime}, k, k^{\prime}, \\
& v_{i k}^{2} \leq v_{i}^{2} \quad \forall i, k, \\
& \sum_{k=1}^{2^{n R_{1}}} v_{i k}^{2} \geq\left(1-\epsilon^{\frac{1}{4}}\right) 2^{n R_{1}} v_{i}^{2} \quad \forall i \\
& \sum_{i=1}^{2^{n R_{2}}} v_{i}^{2} \geq\left(1-\epsilon^{\frac{1}{4}}\right) \cdot 2^{n R_{2}} \\
& v_{i}^{1}, v_{k}^{2}, v_{i k}^{1}, v_{i k}^{2}, v_{i k t}^{1}, v_{i k t}^{2} \in\{0,1\}, \quad \forall i, k, t
\end{aligned}
$$

2. If the problem defined by constraints (4.49)-(4.65) is feasible, then $\left(R_{1}, R_{2}\right)$ is achievable and the resulting codewords can be used to transmit messages. 
Algorithm 3 Decoding Algorithm for two-user Interference Channel.

Input : Received codewords $\mathbf{y}^{1}, \mathbf{y}^{2}$.

Output : Messages $i_{1}^{*}, i_{2}^{*}$.

Algorithm:

1. Solve

$$
\begin{aligned}
& i_{1}^{*}=g^{1}\left(\mathbf{y}^{1}\right)=\arg \min _{i \in\left\{1, \ldots, M_{1}\right\}: v_{i}^{1}=1}\left[\min _{k \in\left\{1, \ldots, M_{2}\right\}: v_{i k}^{1}=1}\left\|\mathbf{y}^{1}-\left(\mathbf{x}_{i}^{1}+h_{12} \mathbf{x}_{k}^{2}\right)\right\|\right], \\
& i_{2}^{*}=g^{2}\left(\mathbf{y}^{2}\right)=\arg \min _{k \in\left\{1, \ldots, M_{2}\right\}: v_{k}^{2}=1}\left[\min _{i \in\left\{1, \ldots, M_{1}\right\}: v_{i k}^{2}=1}\left\|\mathbf{y}^{1}-\left(\mathbf{x}_{i}^{2}+h_{21} \mathbf{x}_{k}^{1}\right)\right\|\right] .
\end{aligned}
$$

In particular, constraints (4.51)-(4.54) ensure that, whenever $v_{i k}^{1}=1$ and $v_{i^{\prime} k^{\prime}}^{1}=1$, then for at least $\left(1-\epsilon^{\frac{1}{4}}\right) T$ of the $\mathbf{z}_{t}$ 's in $\mathcal{Z}$,

$$
\left\|\left(\mathbf{x}_{i}^{1}+h_{12} \mathbf{x}_{k}^{2}\right)-\left(\mathbf{x}_{i^{\prime}}^{1}+h_{12} \mathbf{x}_{k^{\prime}}^{2}\right)+\mathbf{z}_{t}\right\| \geq\left\|\mathbf{z}_{t}\right\|
$$

thus ensuring that for at least $\left(1-\epsilon^{\frac{1}{4}}\right) T$ of the $\mathbf{z}_{t}$ 's in $\mathcal{Z}, \mathbf{x}_{i}^{1}+h_{12} \mathbf{x}_{k}^{2}+\mathbf{z}_{t}$ is decoded as $i$ by using the decoding Algorithm 3. Furthermore, constraints (4.55)-(4.57) ensure that there are at least $\left(1-\epsilon^{\frac{1}{4}}\right) 2^{n R_{1}}$ number of codewords $i \in \mathcal{B}^{1}$, such that for each such codeword $i$, there are at least $\left(1-\epsilon^{\frac{1}{4}}\right) 2^{n R_{2}}$ codewords $k \in \mathcal{B}^{2}$ with the probability of correct decoding of at least $1-\epsilon^{\frac{1}{4}}$. Constraints (4.58)-(4.64) are the corresponding decoding constraints for User 2.

We next provide a reformulation of the feasibility problem (4.49-4.65) into an equivalent rank minimization problem with semidefinite constraints. Let

$$
\mathbf{y}=\left(1, \mathbf{x}_{i}^{1}, \mathbf{x}_{k}^{2}, v_{i}^{1}, v_{k}^{2}, v_{i k}^{1}, v_{i k}^{2}, v_{i k i^{\prime} k^{\prime} t}^{1}, v_{i k i^{\prime} k^{\prime} t}^{2}\right)
$$

be the concatenation in a single vector of all the decisions variables in the feasibility problem (4.49-4.65). Letting $Y=\mathbf{y y}^{\prime}$, note that $\operatorname{rank}(Y)=1$ and $Y \succeq 0$. Using Propositions 27 and 28, we reformulate the feasibility problem (4.49-4.65) as the problem of minimizing the $\operatorname{rank}(Y)$ subject to linear constraints in $Y$ and $Y \succeq 0$. 


$$
\begin{aligned}
& r^{*}=\min \quad \operatorname{rank}(Y) \\
& \text { s.t. } \quad A_{i}^{1} \bullet Y \leq 0, \quad \forall i=1, \ldots, 2^{n R_{1}} \text {, } \\
& A_{k}^{2} \bullet Y \leq 0, \quad \forall k=1, \ldots, 2^{n R_{2}}, \\
& B_{i k i^{\prime} k^{\prime} t}^{1} \bullet Y \leq 0, \quad \forall t=1, \ldots, T, \forall i, k, i^{\prime}, k^{\prime} \\
& v_{i k i^{\prime} k^{\prime} t}^{1} \leq v_{i k}^{1} \\
& v_{i k i^{\prime} k^{\prime} t}^{1} \leq v_{i^{\prime} k^{\prime}}^{1} \\
& \sum_{t=1}^{T} v_{i k i^{\prime} k^{\prime} t}^{1} \geq\left(1-\epsilon^{\frac{1}{4}}\right) T \cdot v_{i k}^{1} v_{i^{\prime} k^{\prime}}^{1} \\
& v_{i k}^{1} \leq v_{i}^{1} \\
& \sum_{k=1}^{2^{n R_{2}}} v_{i k}^{1} \geq\left(1-\epsilon^{\frac{1}{4}}\right) 2^{n R_{2}} \cdot v_{i}^{1} \\
& \sum_{i=1}^{2^{n R_{1}}} v_{i}^{1} \geq\left(1-\epsilon^{\frac{1}{4}}\right) \cdot 2^{n R_{1}} \\
& B_{i k i^{\prime} k^{\prime} t}^{2} \bullet Y \leq 0, \quad \forall t=1, \ldots, T, \forall i, k, i^{\prime}, k^{\prime} \\
& v_{i k i^{\prime} k^{\prime} t}^{2} \leq v_{i k}^{2} \\
& v_{i k i^{\prime} k^{\prime} t}^{2} \leq v_{i^{\prime} k^{\prime}}^{2} \\
& \sum_{t=1}^{T} v_{i k i^{\prime} k^{\prime} t}^{2} \geq\left(1-\epsilon^{\frac{1}{4}}\right) T \cdot v_{i k}^{2} v_{i^{\prime} k^{\prime}}^{2} \\
& Y \succeq 0
\end{aligned}
$$

where

$$
A_{i}^{1}(p, q)= \begin{cases}-n P_{1}, & \text { if } p=1, q=1 \\ 0, & \text { if } p=1, q>1, \\ 0, & \text { if } p>1, q=1, \\ 1, & \text { if } \forall p=q=(i-1) n+1, \ldots, i n+1, \\ 0, & \text { otherwise. }\end{cases}
$$




$$
A_{k}^{2}(p, q)= \begin{cases}-n P_{2}, & \text { if } p=1, q=1 \\ 0, & \text { if } p=1, q>1 \\ 0, & \text { if } p>1, q=1 \\ 1, & \text { if } \forall p=q=(k-1) n+1, \ldots, k n+1 \\ 0, & \text { otherwise. }\end{cases}
$$




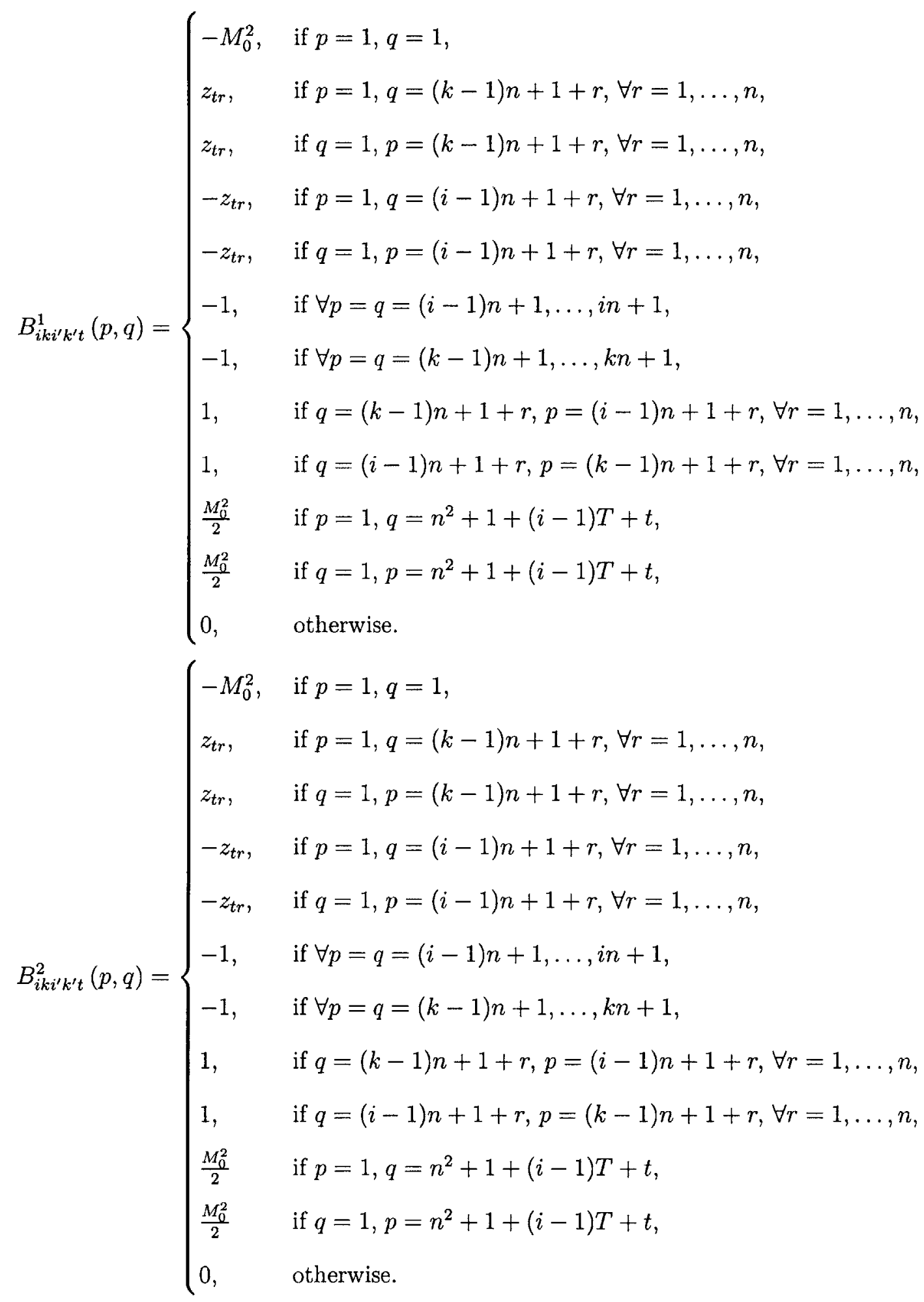


We then use the resulting rank minimization problem with semidefinite constraints in Algorithm 4 that, as we show in Theorem 40, correctly characterizes the capacity region.

Algorithm 4 Capacity Characterization and Optimal Coding for the Two-User Interference Channel

Input : $R_{1}, R_{2}, P_{1}, P_{2}, \sigma, n, \epsilon, \nu$.

Output : Rank $r^{*}$, codewords $\left\{\mathbf{x}_{i}^{1}\right\}_{i=1}^{2^{n R_{1}}},\left\{\mathbf{x}_{k}^{2}\right\}_{k=1}^{2^{n R_{2}}}$, and auxiliary binary variables $v_{i}^{1}, v_{k}^{2}, v_{i k}^{1}, v_{i k}^{2}, v_{i k i^{\prime} k^{\prime} t}^{1}, v_{i k i^{\prime} k^{\prime} t}^{2}$.

\section{Algorithm :}

1. Solve the rank minimization semidefinite optimization problem (4.67) to compute $r^{*}$, codewords $\left\{\mathbf{x}_{i}^{1}\right\}_{i=1}^{2^{n R_{1}}},\left\{\mathbf{x}_{k}^{2}\right\}_{k=1}^{2^{n R_{2}}}$, and auxiliary binary variables $v_{i}^{1}, v_{k}^{2}, v_{i k}^{1}, v_{i k}^{2}, v_{i k i^{\prime} k^{\prime} t}^{1}, v_{i k i^{\prime} k^{\prime} t}^{2}$.

2. If $r^{*}=1$, then $\left(R_{1}, R_{2}\right)$ is achievable using the codebooks $\mathcal{B}^{1}=\left\{\mathbf{x}_{i}^{1}\right\}_{i=1}^{2^{n R_{1}}}$, $\mathcal{B}^{2}=\left\{\mathbf{x}_{k}^{2}\right\}_{k=1}^{n^{n R_{2}}}$ and the decoding functions (4.66), achieving an average decoding error probability of $3 \epsilon^{\frac{1}{4}}+\epsilon$. That is,

$$
\text { If } r^{*}=1 \text {, then }\left(R_{1}, R_{2}\right) \in \mathcal{R}_{n}^{\text {IC }}\left[P_{1}, P_{2}, \sigma, 3 \epsilon^{\frac{1}{4}}+\epsilon\right] \text {. }
$$

3. If $r^{*} \geq 2$, then we can conclude using Theorem 40 that, $\left(R_{1}, R_{2}\right)$ cannot be achieved on a Gaussian interference channel with noise standard deviation $(1+$ $2 \nu) \sigma$ with probability of error less than or equal to $\epsilon(1-\delta(\nu, n))$. That is,

$$
\text { If } r^{*} \geq 2, \text { then }\left(R_{1}, R_{2}\right) \notin \mathcal{R}_{n}^{\mathrm{IC}}\left[P_{1}, P_{2},(1+3 \nu) \sigma, \epsilon(1-\delta(\nu, n))\right]
$$

where

$$
\begin{aligned}
\delta(\nu, n) & =\exp \left(-n \cdot \frac{r-\log (1+r)}{2(1+3 \nu)^{2} \sigma^{2}}\right), \text { with } r=\sigma^{2}\left((1+3 \nu)^{2}-(1+2 \nu)^{2}\right) \\
& \rightarrow 0, \text { as } n \rightarrow \infty
\end{aligned}
$$




\subsubsection{Correctness of Algorithm 4}

The proof of correctness is similar to the single user case. We let $\mathscr{E}_{i k}^{1}[\tilde{\mathbf{z}}]$ denote the event that a decoding error occurs when User 1 sends message $i$ and User 2 sends message $k$ on the channel and noise vector $\tilde{\mathbf{z}}$ is realized, that is,

$$
\mathscr{E}_{i k}^{1}[\tilde{\mathbf{z}}]=\left\{\exists i^{\prime} \neq i, k^{\prime}:\left\|\left(\mathbf{x}_{i}^{1}+h_{12} \mathbf{x}_{k}^{2}\right)-\left(\mathbf{x}_{i^{\prime}}^{1}+h_{12} \mathbf{x}_{k^{\prime}}^{2}\right)+\tilde{\mathbf{z}}\right\| \leq\|\tilde{\mathbf{z}}\|\right\}
$$

Let $1\left\{\mathscr{E}_{i k}^{1}[\tilde{\mathbf{z}}]\right\}$ denote the indicator random variable corresponding to $\mathscr{E}_{i k}^{1}[\tilde{\mathbf{z}}]$, that is,

$$
1\left\{\mathscr{E}_{i k}^{1}[\tilde{\mathbf{z}}]\right\}= \begin{cases}1, & \text { if } \exists i^{\prime} \neq i, k^{\prime}:\left\|\left(\mathbf{x}_{i}^{1}+h_{12} \mathbf{x}_{k}^{2}\right)-\left(\mathbf{x}_{i^{\prime}}^{1}+h_{12} \mathbf{x}_{k^{\prime}}^{2}\right)+\tilde{\mathbf{z}}\right\| \leq\|\tilde{\mathbf{z}}\| \\ 0, & \text { otherwise. }\end{cases}
$$

We begin by obtaining the following proposition which are integral to the proof of our characterization of the capacity region.

\section{Proposition 35.}

Consider any sequence of non-negative numbers $\left\{a_{1}, a_{2}, \ldots, a_{N}\right\}$ such that $\sum_{i=1}^{N} a_{i} \leq$ $N \alpha$. Then, for each fraction $f \in(0,1)$, there exists a subset $\mathcal{A} \subseteq\left\{a_{1}, a_{2}, \ldots, a_{N}\right\}$ of size at least $f \cdot N$ such that

$$
a_{i} \leq \alpha \cdot \frac{1}{1-f}, \forall i \in \mathcal{A}
$$

Proof. Without loss of generality, we assume that $f \cdot N$ is an integer and that the numbers $\left\{a_{1}, a_{2}, \ldots, a_{N}\right\}$ are sorted such that

$$
a_{1} \leq a_{2} \leq \ldots \leq a_{N}
$$

It suffices to show that $a_{f N} \leq \alpha \cdot \frac{1}{1-f}$. We consider the following linear optimization 
problem

$$
\begin{array}{ll}
\max \quad x_{f N} & \\
\text { s.t. } & \sum_{i=1}^{N} x_{i} \leq \alpha N, \\
x_{f N} \geq x_{i}, \quad \forall i<f N, \\
x_{f N} \leq x_{i}, \quad \forall i>f N, \\
x_{i} \geq 0, \quad \forall i,
\end{array}
$$

whose dual problem is given by

$$
\begin{array}{ccc}
\min & \alpha N q & \\
\text { s.t. } & q+p_{i} \geq 0, & \forall i<f N, \\
q-p_{i} \geq 0, & \forall i>f N, \\
& q+\sum_{i>f N} p_{i}-\sum_{i<f N} p_{i} \geq 1, & \\
p_{i} \geq 0 . &
\end{array}
$$

Consider the following solution to (4.68) and (4.69)

$$
\begin{aligned}
& x_{i}^{*}=0, \forall i<f N, \\
& x_{i}^{*}=\frac{\alpha}{1-f}, \forall i \geq f N, \\
& q^{*}=\frac{1}{N(1-f)}, \\
& p_{i}^{*}=q^{*}, \forall i>f N, \\
& p_{i}^{*}=0, \quad \forall i<f N .
\end{aligned}
$$

It is easy to verify that $\left\{x_{i}^{*}\right\}_{i=1}^{N}$ is feasible to (4.68), and $q^{*},\left\{p_{i}^{*}\right\}_{i=1}^{N}$ is feasible to (4.69) with the same objective value. Therefore, an optimal solution to (4.68) will satisfy $x_{i}^{*}=0, \forall i<f N$ and $x_{i}^{*}=x_{f N}^{*}, \forall i>f N$ and thus $x_{f N}^{*}=\alpha \cdot \frac{1}{1-f}$.

Another result we use is due to Shannon [1948a] that essentially says that the 
minimum distance decoder is optimal for single user Gaussian channels.

Proposition 36 (Shannon [1948a]). Let $\mathcal{C}$ be a code with codebook $\mathcal{B}$ with some decoding function $g(\cdot)$, that achieves an average decoding error probability of less than or equal to $\epsilon$ on a Gaussian channel with mean 0 and standard deviation $\Lambda$. Then switching from $g(\cdot)$ to the minimum distance decoder with the same codebook $\mathcal{B}$ also achieves a decoding error probability of less than or equal to $\epsilon$.

We next examine certain properties of optimal solutions $\left\{\mathrm{x}_{i}^{1}\right\}_{i=1}^{2^{n R_{1}}},\left\{\mathrm{x}_{k}^{2}\right\}_{k=1}^{2^{n R_{2}}}$ of (4.67).

Proposition 37. Let $A, B$ and $C$ be three channels with noise vectors $\tilde{\mathbf{u}}_{A}, \tilde{\mathbf{u}}_{B}, \tilde{\mathbf{z}}_{C}$ where $\tilde{\boldsymbol{u}}_{A}, \tilde{\boldsymbol{u}}_{B}$ are distributed uniformly on the surface of the $n$-spheres $\mathcal{S}_{n}\left(r_{A}\right)$ and $\mathcal{S}_{n}\left(r_{B}\right)$, respectively, and $\tilde{z}_{C} \sim N(\mathbf{0}, \gamma \mathbf{e})$. Let $\left\{\mathbf{x}_{i}^{1}\right\}_{i=1}^{2^{n R_{1}}},\left\{\mathbf{x}_{k}^{2}\right\}_{k=1}^{2^{n R_{2}}}$ be the set of codewords, and let $\mathbf{v}_{i k}=\mathbf{x}_{i}^{1}+h_{12} \mathbf{x}_{k}^{2}$. Then,

(a) If $r_{A}>r_{B}$, then

$$
\mathbb{P}\left[\exists i^{\prime} \neq i, k^{\prime}:\left\|\mathbf{v}_{i k}-\mathbf{v}_{i^{\prime} k^{\prime}}+\tilde{\mathbf{u}}_{A}\right\| \leq r_{A}\right] \geq \mathbb{P}\left[\exists i^{\prime} \neq i, k^{\prime}:\left\|\mathbf{v}_{i k}-\mathbf{v}_{i^{\prime} k^{\prime}}+\tilde{\mathbf{u}}_{B}\right\| \leq r_{B}\right]
$$

(b) $\mathbb{P}\left[\exists i^{\prime} \neq i, k^{\prime}:\left\|\mathbf{v}_{i k}-\mathbf{v}_{i^{\prime} k^{\prime}}+\tilde{u}_{A}\right\| \leq r_{A}\right] \geq$

$$
\mathbb{P}\left[\exists i^{\prime} \neq i, k^{\prime}:\left\|\mathbf{v}_{i k}-\mathbf{v}_{i^{\prime} k^{\prime}}+\tilde{z}_{C}\right\| \leq\left\|\tilde{z}_{C}\right\| \mid\left\|\tilde{z}_{C}\right\| \leq r_{A}\right]
$$

(c) $\mathbb{P}\left[\exists i^{\prime} \neq i, k^{\prime}:\left\|\mathbf{v}_{i k}-\mathbf{v}_{i^{\prime} k^{\prime}}+\tilde{u}_{A}\right\| \leq r_{A}\right] \leq$

$$
\mathbb{P}\left[\exists i^{\prime} \neq i, k^{\prime}:\left\|\mathbf{v}_{i k}-\mathbf{v}_{i^{\prime} k^{\prime}}+\tilde{\boldsymbol{z}}_{C}\right\| \leq\left\|\tilde{\boldsymbol{z}}_{C}\right\|\|\| \tilde{\boldsymbol{z}}_{C} \|>r_{A}\right]
$$

Proof. Applying Proposition 31 with $\mathbf{x}_{i}=\mathbf{v}_{i k}, \mathbf{x}_{k}=\mathbf{v}_{i^{\prime} k^{\prime}}$, each of these statements follow.

Proposition 38. Let $\mathcal{C}$ be a code with codebook $\mathcal{B}$ and a minimum distance decoding function. Consider two noises $\tilde{\mathbf{z}}_{G} \sim N(\mathbf{0}, \sigma e)$ and $\tilde{\mathbf{z}}_{U}$ uniformly distributed on the ball $\mathcal{S}_{n}\left(\sigma^{\prime} \sqrt{n}\right)$ with $\sigma^{\prime}<\sigma$. If $\mathbb{P}\left[\mathscr{E}_{i k}^{1}\left[\tilde{\mathbf{z}}_{G}\right]\right] \leq \epsilon, \forall i \in \mathcal{B}$, then

$$
\mathbb{P}\left[\mathscr{E}_{i k}^{1}\left[\tilde{\mathbf{z}}_{U}\right]\right] \leq \frac{\epsilon}{1-\exp (-n \beta)}, \text { with } \beta=\frac{\sigma^{2}-\sigma^{\prime 2}-\log \left(1+\sigma^{2}-\sigma^{2}\right)}{2 \sigma^{2}}
$$


Proof. The proof is very similar to that of Proposition 32 and is omitted.

Proposition 39. Let $\tilde{\mathbf{s}}$ be a uniformly chosen in $\mathcal{S}_{n}\left(\gamma_{\epsilon}\right)$. Let $\left\{\mathbf{x}_{i}^{1}\right\}_{i=1}^{2^{n R_{1}}},\left\{\mathbf{x}_{k}^{2}\right\}_{k=1}^{2^{n R_{2}}}$ be the set of codewords, and let $\mathbf{v}_{i k}=\mathbf{x}_{i}^{1}+h_{12} \mathbf{x}_{k}^{2}$. Then,

(a) $\mathbb{P}\left[\exists i^{\prime} \neq i, k^{\prime}:\left\|\mathbf{v}_{i k}-\mathbf{v}_{i^{\prime} k^{\prime}}+\tilde{\mathbf{s}}\right\| \geq \gamma_{\epsilon}\right] \geq \mathbb{P}\left[v_{i k r(\tilde{\mathbf{s}})}^{1}=1\right]$,

(b) $\mathbb{P}\left[v_{i k \tau(\tilde{\mathbf{s}})}^{1}=1\right] \geq 1-\epsilon^{\frac{1}{4}}$.

Proof.

(a) Constraint (4.51) implies that $\left\{\mathrm{x}_{i}^{1}\right\}_{i=1}^{2^{n R_{1}}},\left\{\mathrm{x}_{k}^{2}\right\}_{k=1}^{2^{n R_{2}}}$ satisfy that, for the noise vector $\mathbf{z}_{t}^{\prime}$ 's in $\mathcal{Z}$ with $v_{i k t}^{\mathrm{I}}=1$,

$$
\begin{aligned}
\left\|\mathbf{v}_{i k}-\mathbf{v}_{i^{\prime} k^{\prime}}+\mathbf{z}_{t}\right\| & \geq\left\|\mathbf{z}_{t}\right\|, \forall k \neq i, \Longleftrightarrow\left\|\mathbf{v}_{i k}-\mathbf{v}_{i^{\prime} k^{\prime}}\right\|^{2} \geq 2\left\langle\mathbf{v}_{i^{\prime} k^{\prime}}-\mathbf{v}_{i k}, \mathbf{z}_{t}\right\rangle, \forall k \neq i \\
& \Longleftrightarrow\left\|\mathbf{v}_{i k}-\mathbf{v}_{i^{\prime} k^{\prime}}\right\|^{2} \geq 2(1+\nu)\left\langle\mathbf{v}_{i^{\prime} k^{\prime}}-\mathbf{v}_{i k}, \mathbf{w}_{t}\right\rangle, \forall k \neq i .
\end{aligned}
$$

We also have, given that $\zeta=\sigma \Phi^{-1}\left(1-\epsilon^{\frac{1}{4}}\right) / \sqrt{n}$,

$$
\left\|\mathbf{v}_{i k}-\mathbf{v}_{i^{\prime} k^{\prime}}\right\| \geq 2 \sigma \Phi^{-1}\left(1-\epsilon^{\frac{1}{4}}\right) \Longrightarrow\left\|\mathbf{v}_{i k}-\mathbf{v}_{i^{\prime} k^{\prime}}\right\| \geq 2 \sqrt{n} \zeta
$$

Since $\left\{\mathbf{z}_{1}, \mathbf{z}_{2}, \ldots, \mathbf{z}_{T}\right\}$ forms a Voronoi tessellation of $\mathcal{S}_{n}\left((1+\nu) \gamma_{\epsilon}\right)$, the set $\mathcal{W}$ also forms a Voronoi tessellation on $\mathcal{S}_{n}\left(\gamma_{\epsilon}\right)$. Therefore, letting $\mathbf{s}$ be any vector belonging to $\mathcal{S}^{n}$, from Proposition 29 applied to $\mathcal{A}=\mathcal{W}, N=T, \Lambda=\gamma_{\epsilon}$, we obtain

$$
\left\|\mathbf{s}-\mathbf{w}_{\tau(\tilde{\mathbf{s}})}\right\| \leq \theta \sqrt{n}
$$

where $\tau(\mathbf{s})=\arg \min _{t=1, \ldots, T}\left\|\mathbf{s}-\mathbf{w}_{t}\right\|$, with

$$
\theta=\frac{\gamma_{\epsilon}}{\sqrt{n} T^{1 / n}}=\frac{\zeta \nu}{1+\nu}
$$

From (4.73) and (4.71), we have

$$
2 \theta \sqrt{n} \leq \frac{\nu}{1+\nu} \cdot\left\|\mathbf{v}_{i k}-\mathbf{v}_{i^{\prime} k^{\prime}}\right\|
$$


We now proceed to show that if $v_{i k \tau(\mathbf{s})}^{1}=1$, then $\left\|\mathbf{v}_{i k}-\mathbf{v}_{i^{\prime} k^{\prime}}+\mathbf{s}\right\| \geq\|\mathbf{s}\|$. We have

$$
\begin{aligned}
2\left\langle\mathbf{v}_{i^{\prime} k^{\prime}}-\mathbf{v}_{i k}, \mathbf{s}\right\rangle & =2\left\langle\mathbf{v}_{i^{\prime} k^{\prime}}-\mathbf{v}_{i k},\left(\mathbf{s}-\mathbf{w}_{\tau}\right)+\mathbf{w}_{\tau}\right\rangle \\
& \leq 2\left\langle\mathbf{v}_{i^{\prime} k^{\prime}}-\mathbf{v}_{i k}, \mathbf{w}_{\tau}\right\rangle+2 \cdot\left\|\mathbf{v}_{i^{\prime} k^{\prime}}-\mathbf{v}_{i k}\right\| \cdot\left\|\mathbf{s}-\mathbf{w}_{\tau}\right\| \\
& \leq(1+\nu)^{-1}\left\|\mathbf{v}_{i^{\prime} k^{\prime}}-\mathbf{v}_{i k}\right\|^{2}+2 \cdot\left\|\mathbf{v}_{i^{\prime} k^{\prime}}-\mathbf{v}_{i k}\right\| \cdot \theta \sqrt{n} \text { (from (4.72)) } \\
& =\left\|\mathbf{v}_{i k}-\mathbf{v}_{i^{\prime} k^{\prime}}\right\| \cdot\left\{(1+\nu)^{-1}\left\|\mathbf{v}_{i k}-\mathbf{v}_{i^{\prime} k^{\prime}}\right\|+2 \theta \sqrt{n}\right\} \\
& \leq\left\|\mathbf{v}_{i k}-\mathbf{v}_{i^{\prime} k^{\prime}}\right\| \cdot\left\{(1+\nu)^{-1}\left\|\mathbf{v}_{i k}-\mathbf{v}_{i^{\prime} k^{\prime}}\right\|+\frac{\nu}{1+\nu}\left\|\mathbf{v}_{i k}-\mathbf{v}_{i^{\prime} k^{\prime}}\right\|\right\} \\
& =\left\|\mathbf{v}_{i k}-\mathbf{v}_{i^{\prime} k^{\prime}}\right\|^{2}
\end{aligned}
$$

Therefore, if $v_{i k \tau(\mathrm{s})}^{1}=1$, then

$$
2\left\langle\mathbf{v}_{i^{\prime} k^{\prime}}-\mathbf{v}_{i k}, \mathbf{s}\right\rangle \leq\left\|\mathbf{v}_{i k}-\mathbf{v}_{i^{\prime} k^{\prime}}\right\|^{2} \Longleftrightarrow\left\|\mathbf{v}_{i k}-\mathbf{v}_{i^{\prime} k^{\prime}}+\mathbf{s}\right\| \geq\|\mathbf{s}\|
$$

This implies that $\mathbb{P}\left[\exists i^{\prime} \neq i, k^{\prime}:\left\|\mathbf{v}_{i k}-\mathbf{v}_{i^{\prime} k^{\prime}}+\tilde{\mathbf{s}}\right\| \geq \gamma_{\epsilon}\right] \geq \mathbb{P}\left[v_{i k \tau(\tilde{\mathbf{s}})}^{1}=1\right]$.

(b) Let $\tilde{\mathbf{s}}$ be chosen uniformly at random on $\mathcal{S}_{n}\left(\gamma_{\epsilon}\right)$. We have

$$
\mathbb{P}\left[v_{i k \tau(\tilde{\mathbf{s}})}^{1}=1\right]=\sum_{t=1}^{T} \mathbb{P}\left[v_{i k \tau(\tilde{\mathbf{s}})}^{1}=1 \mid \tau(\tilde{\mathbf{s}})=t\right] \cdot \mathbb{P}[\tau(\tilde{\mathbf{s}})=t]
$$

We have

$$
\begin{aligned}
\mathbb{P}\left[v_{i k \tau(\overline{\mathbf{s}})}^{1}=1 \mid \tau(\tilde{\mathbf{s}})=t\right] & = \begin{cases}1, & \text { if } v_{i k t}^{1}=1 \\
0, & \text { otherwise. }\end{cases} \\
& =v_{i k t}^{1} .
\end{aligned}
$$

Moreover, since the set of vectors $\mathcal{W}$ form a Voronoi tessellation, the Voronoi regions of the points $\mathbf{w}_{t} \in \mathcal{W}$ are identical with the same area. Consequently, choosing $\tilde{\mathbf{s}}$ uniformly at random induces a uniform distribution for $\tau(\tilde{\mathbf{s}})$ on the elements of the set $\{1, \ldots, T\}$, that is,

$$
\mathbb{P}[\tau(\tilde{\mathbf{s}})=t]=\frac{1}{T}, \forall t=1, \ldots, T
$$


Substituting (4.76) and (4.77) in (4.75), we have

$$
\begin{aligned}
\mathbb{P}\left[v_{i k \tau(\tilde{\mathbf{s}})}^{1}=1\right] & =\sum_{t=1}^{T} \mathbb{P}\left[v_{i k \tau(\tilde{\mathbf{s}})}^{1}=1 \mid \tau(\tilde{\mathbf{s}})=t\right] \cdot \mathbb{P}[\tau(\tilde{\mathbf{s}})=t] \\
& =\frac{1}{T} \sum_{t=1}^{T} v_{i k t}^{1} \geq \frac{1}{T} \cdot\left(1-\epsilon^{\frac{1}{4}}\right) T \quad(\text { from }(4 . \\
& =1-\epsilon^{\frac{1}{4}} .
\end{aligned}
$$

We are now in a position to present our main result.

Theorem 40. (Capacity Region in a Two User Gaussian Interference

\section{Channel)}

(a) If $r^{*}=1$, then $\left(R_{1}, R_{2}\right) \in \mathcal{R}_{n}^{I C}\left[P_{1}, P_{2}, \sigma, 3 \epsilon^{\frac{1}{4}}+\epsilon\right]$, that is, $\left(R_{1}, R_{2}\right)$ is achievable using the codebooks $\mathcal{B}^{1}=\left\{\mathbf{x}_{i}^{1}\right\}_{i=1}^{2^{n R_{1}}}, \mathcal{B}^{2}=\left\{\mathrm{x}_{k}^{2}\right\}_{k=1}^{2^{n R_{2}}}$ and the decoding functions (4.66), achieving an average decoding error probability of $3 \epsilon^{\frac{1}{4}}+\epsilon$.

(b) If $r^{*} \geq 2$, then $\left(R_{1}, R_{2}\right) \notin \mathcal{R}_{n}^{I C}\left[P_{1}, P_{2},(1+3 \nu) \sigma, \epsilon(1-\delta(\nu, n))\right]$, where

$$
\begin{aligned}
\delta(\nu, n) & =\exp \left(-n \cdot \frac{r-\log (1+r)}{2(1+3 \nu)^{2} \sigma^{2}}\right), \text { with } r=\sigma^{2}\left((1+3 \nu)^{2}-(1+2 \nu)^{2}\right) \\
& \rightarrow 0, \text { as } n \rightarrow \infty
\end{aligned}
$$

Proof. (a) We consider a Gaussian channel with noise $\tilde{\mathbf{z}}_{G}$ with each component being a Gaussian random variable with mean 0 and standard deviation $\sigma$. We also have where $\mathcal{U}_{\epsilon}^{\text {Normal }}=\left\{\mathrm{s} \in \mathbb{R}^{n} \mid\|\mathbf{s}\| \leq \gamma_{\epsilon}\right\}$, with $\gamma_{\epsilon}=\sqrt{n \sigma^{2}+\Gamma_{\epsilon}}$. Consider the probability of error for User 1 , when User 1 sends message $i$ and User 2 sends message $k$

$$
\begin{aligned}
\mathbb{P}\left[\mathscr{E}_{i k}^{1}\left[\tilde{\mathbf{z}}_{G}\right]\right]= & \mathbb{P}\left[\exists i^{\prime} \neq i, k^{\prime}:\left\|\left(\mathbf{x}_{i}^{1}+h_{12} \mathbf{x}_{k}^{2}\right)-\left(\mathbf{x}_{i^{\prime}}^{1}+h_{12} \mathbf{x}_{k^{\prime}}^{2}\right)+\tilde{\mathbf{z}}_{G}\right\| \leq \tilde{\mathbf{z}}_{G}\right] \\
= & \int_{0}^{\gamma_{\epsilon}} \mathbb{P}\left[\exists i^{\prime} \neq i, k^{\prime}:\left\|\mathbf{v}_{i k}-\mathbf{v}_{i^{\prime} k^{\prime}}+\tilde{\mathbf{z}}_{G}\right\| \leq \tilde{\mathbf{z}}_{G},\left\|\tilde{\mathbf{z}}_{G}\right\| \in(c-\mathrm{d} c, c]\right] \mathrm{d} c \\
& +\int_{\gamma_{\epsilon}}^{\infty} \mathbb{P}\left[\exists i^{\prime} \neq i, k^{\prime}:\left\|\mathbf{v}_{i k}-\mathbf{v}_{i^{\prime} k^{\prime}}+\tilde{\mathbf{z}}_{G}\right\| \leq \tilde{\mathbf{z}}_{G},\left\|\tilde{\mathbf{z}}_{G}\right\| \in(c-\mathrm{d} c, c]\right] \mathrm{d} c
\end{aligned}
$$


where $\mathbf{v}_{i k}=\mathbf{x}_{i}^{1}+h_{12} \mathbf{x}_{k}^{2}$. The second term can be bounded as follows:

$$
\begin{gathered}
\int_{\gamma_{\epsilon}}^{\infty} \mathbb{P}\left[\exists i^{\prime} \neq i, k^{\prime}:\left\|\mathbf{v}_{i k}-\mathbf{v}_{i^{\prime} k^{\prime}}+\tilde{\mathbf{z}}_{G}\right\| \leq\left\|\tilde{\mathbf{z}}_{G}\right\|,\left\|\tilde{\mathbf{z}}_{G}\right\| \in(c-\mathrm{d} c, c]\right] \mathrm{d} c \\
\quad \leq \int_{\gamma_{\epsilon}}^{\infty} \mathbb{P}\left[\left\|\tilde{\mathbf{z}}_{G}\right\| \in(c-\mathrm{d} c, c]\right] \mathrm{d} c=\mathbb{P}\left[\tilde{\mathbf{z}}_{G} \notin \mathcal{U}_{\epsilon}^{\text {Normal }}\right] \leq \epsilon
\end{gathered}
$$

We next bound the first term as follows,

$$
\begin{aligned}
\int_{0}^{\gamma_{\epsilon}} & \mathbb{P}\left[\exists i^{\prime} \neq i, k^{\prime}:\left\|\mathbf{v}_{i k}-\mathbf{v}_{i^{\prime} k^{\prime}}+\tilde{\mathbf{z}}_{G}\right\| \leq\left\|\tilde{\mathbf{z}}_{G}\right\|,\left\|\tilde{\mathbf{z}}_{G}\right\| \in(c-\mathrm{d} c, c]\right] \mathrm{d} c \\
= & \int_{0}^{\gamma_{\epsilon}} \mathbb{P}\left[\exists i^{\prime} \neq i, k^{\prime}:\left\|\mathbf{v}_{i k}-\mathbf{v}_{i^{\prime} k^{\prime}}+\tilde{\mathbf{z}}_{G}\right\| \leq\left\|\tilde{\mathbf{z}}_{G}\right\|\left\|\tilde{\mathbf{z}}_{G}\right\| \in(c-\mathrm{d} c, c]\right] \\
& \cdot \mathbb{P}\left[\left\|\tilde{\mathbf{z}}_{G}\right\| \in(c-\mathrm{d} c, c]\right] \mathrm{d} c \\
\leq & \int_{0}^{\gamma_{\epsilon}} \mathbb{P}\left[\exists i^{\prime} \neq i, k^{\prime}:\left\|\mathbf{v}_{i k}-\mathbf{v}_{i^{\prime} k^{\prime}}+\tilde{\mathbf{s}}(c)\right\| \leq c\right] \cdot \mathbb{P}\left[\left\|\tilde{\mathbf{z}}_{G}\right\| \in(c-\mathrm{d} c, c]\right] \mathrm{d} c \\
\leq & \int_{0}^{\gamma_{\epsilon}} \mathbb{P}\left[\exists i^{\prime} \neq i, k^{\prime}:\left\|\mathbf{v}_{i k}-\mathbf{v}_{i^{\prime} k^{\prime}}+\tilde{\mathbf{s}}\left(\gamma_{\epsilon}\right)\right\| \leq \gamma_{\epsilon}\right] \cdot \mathbb{P}\left[\left\|\tilde{\mathbf{z}}_{G}\right\| \in(c-\mathrm{d} c, c]\right] \mathrm{d} c \\
\leq & \int_{0}^{\gamma_{\epsilon}}\left(1-\mathbb{P}\left[v_{i k \tau_{\tilde{\mathbf{s}}\left(\gamma_{\epsilon}\right)}}=1\right]\right) \cdot \mathbb{P}\left[\left\|\tilde{\mathbf{z}}_{G}\right\| \in(c-\mathrm{d} c, c]\right] \mathrm{d} c \\
\leq & \int_{0}^{\gamma_{\epsilon}}\left(1-\left(1-\epsilon^{\frac{1}{4}}\right)\right) \cdot \mathbb{P}\left[\left\|\tilde{\mathbf{z}}_{G}\right\| \in(c-\mathrm{d} c, c]\right] \mathrm{d} c \\
\leq & \epsilon^{\frac{1}{4}} \cdot \int_{0}^{\gamma_{\epsilon}} \mathbb{P}\left[\left\|\tilde{\mathbf{z}}_{G}\right\| \in(c-\mathrm{d} c, c]\right] \mathrm{d} c \leq \epsilon^{\frac{1}{4}} .
\end{aligned}
$$

From (4.35) and (4.36), we have

$$
\mathbb{P}\left[\mathscr{E}_{i k}^{1}\left[\tilde{\boldsymbol{z}}_{G}\right]\right] \leq \epsilon+\epsilon^{\frac{1}{4}}
$$


Proceeding further, we have

$$
\begin{aligned}
\mathbb{P}\left[g^{1}\left(\mathbf{y}^{1}\right) \neq i \mid m^{1}=i\right] & =\frac{1}{2^{n R_{2}}} \sum_{k=1}^{2^{n R_{2}}} \mathbb{P}\left[\mathscr{E}_{i k}^{1}\left[\tilde{\boldsymbol{z}}_{G}\right]\right] \\
& =\frac{1}{2^{n R_{2}}}\left(\sum_{k: v_{i k}^{1}=1} \mathbb{P}\left[\mathscr{E}_{i k}^{1}\left[\tilde{z}_{G}\right]\right]+\sum_{k: v_{i k}^{1}=0} \mathbb{P}\left[\mathscr{E}_{i k}^{1}\left[\tilde{z}_{G}\right]\right]\right) \\
(\text { from }(4.79)) & \leq \frac{1}{2^{n R_{2}}}\left(\left|\left\{k: v_{i k}^{1}=1\right\}\right| \cdot\left(\epsilon+\epsilon^{\frac{1}{4}}\right)+\left|\left\{k: v_{i k}^{1}=0\right\}\right| \cdot 1\right) \\
(\text { from }(4.56)) & \leq \frac{1}{2^{n R_{2}}}\left(2^{n R_{2}} \cdot\left(\epsilon+\epsilon^{\frac{1}{4}}\right)+\epsilon^{\frac{1}{4}} \cdot 2^{n R_{2}} \cdot 1\right) \\
& =2 \epsilon^{\frac{1}{4}}+\epsilon .
\end{aligned}
$$

Next, the average decoding error probability of the code is given by

$$
\begin{aligned}
\mathbb{P}_{\mathrm{avg}} & =\frac{1}{2^{n R_{1}}} \sum_{i=1}^{2^{n R_{1}}} \mathbb{P}\left[g^{1}\left(\mathbf{y}^{1}\right) \neq i \mid m^{1}=i\right] \\
& =\frac{1}{2^{n R_{1}}}\left(\sum_{i: v_{i}^{1}=1} \mathbb{P}\left[g^{1}\left(\mathbf{y}^{1}\right) \neq i \mid m^{1}=i\right]+\sum_{i: v_{i}^{1}=0} \mathbb{P}\left[g^{1}\left(\mathbf{y}^{1}\right) \neq i \mid m^{1}=i\right]\right) \\
& \leq \frac{1}{2^{n R_{1}}}\left(\left|\left\{i: v_{i}^{1}=1\right\}\right| \cdot\left(2 \epsilon^{\frac{1}{4}}+\epsilon\right)+\left|\left\{i: v_{i}^{1}=0\right\}\right| \cdot 1\right) \\
\text { (from (4.57)) } & \leq \frac{1}{2^{n R_{1}}}\left(2^{n R_{1}} \cdot\left(2 \epsilon^{\frac{1}{4}}+\epsilon\right)+\left(1-\left(1-\epsilon^{\frac{1}{4}}\right)\right) \cdot 2^{n R_{1}} \cdot 1\right) \\
& =3 \epsilon^{\frac{1}{4}}+\epsilon .
\end{aligned}
$$

Therefore, $\left(R_{1}, R_{2}\right) \in \mathcal{R}_{n}^{\mathrm{IC}}\left[P_{1}, P_{2}, \sigma, 3 \epsilon^{\frac{1}{4}}+\epsilon\right]$.

(b) We will show that if $\left(R_{1}, R_{2}\right) \in \mathcal{R}_{n}^{\mathrm{IC}}\left[P_{1}, P_{2},(1+3 \nu) \sigma, \epsilon(1-\delta(\nu, n))\right]$, then $r^{*}=$ 1. Consider a rate pair $\left(R_{1}, R_{2}\right) \in \mathcal{R}_{n}^{\mathrm{IC}}\left[P_{1}, P_{2},(1+3 \nu) \sigma, \epsilon(1-\delta(\nu, n))\right]$. By definition, there exists a code $\mathcal{C}$ with codebooks $\mathcal{B}^{1}$ and $\mathcal{B}^{2}$ and a decoding function $g(\cdot)$, that achieves an average error-probability of $\epsilon^{\prime}=\epsilon(1-\delta(\nu, n))$ for each User 1 and 
2 , that is,

$$
\begin{gathered}
\frac{1}{2^{n R_{1}}} \sum_{i=1}^{2^{n R_{1}}} \mathbb{P}\left[g\left(\mathrm{y}^{1}\right) \neq i \mid m^{1}=i\right] \leq \epsilon^{\prime}(1-\delta(\nu, n)), \\
\frac{1}{2^{n R_{2}}} \sum_{k=1}^{2^{n R_{2}}} \mathbb{P}\left[g\left(\mathrm{y}^{2}\right) \neq k \mid m^{2}=k\right] \leq \epsilon^{\prime}(1-\delta(\nu, n)) .
\end{gathered}
$$

In what follows, we restrict ourselves to the analysis of User 1. Applying Proposition 35 with

$$
f=1-\epsilon^{\frac{1}{4}}, N=2^{n R_{1}}, \alpha=\epsilon(1-\delta(\nu, n)), \text { and } a_{i}=\mathbb{P}\left[g\left(\mathbf{y}^{1}\right) \neq i \mid m^{1}=i\right], \forall i
$$

we obtain that there exists a subset of codewords $\mathcal{F}^{1} \subseteq \mathcal{B}^{1}$ such that

$$
\begin{gathered}
\mathbb{P}\left[g\left(\mathbf{y}^{1}\right) \neq i \mid m^{1}=i\right] \leq \frac{\epsilon(1-\delta(\nu, n))}{1-\left(1-\epsilon^{\frac{1}{4}}\right)}=\epsilon^{\frac{3}{4}}(1-\delta(\nu, n)), \forall i \in \mathcal{F}^{1}, \\
\left|\mathcal{F}^{1}\right| \geq\left(1-\epsilon^{\frac{1}{4}}\right) 2^{n R_{1}}
\end{gathered}
$$

Fixing some $i \in \mathcal{F}^{1}$, we have

$$
\mathbb{P}\left[g\left(\mathbf{y}^{1}\right) \neq i \mid m^{1}=i\right]=\frac{1}{2^{n R_{2}}} \sum_{k=1}^{2^{n R_{2}}} \mathbb{P}\left[g\left(\mathbf{y}^{1}\right) \neq i \mid m^{1}=i, m^{2}=k\right] \leq \epsilon^{\frac{3}{4}}(1-\delta(\nu, n))
$$

Applying Proposition 35 with

$$
\begin{gathered}
f=1-\epsilon^{\frac{1}{4}}, N=2^{n R_{2}}, \alpha=\epsilon^{\frac{3}{4}}(1-\delta(\nu, n)) \\
a_{k}=\mathbb{P}\left[g\left(\mathbf{y}^{1}\right) \neq i \mid m^{1}=i, m^{2}=k\right], \forall k=1, \ldots, 2^{n R_{2}},
\end{gathered}
$$


we obtain that there exists a subset of codewords $\mathcal{B}_{i}^{1,2} \subseteq \mathcal{B}^{2}$ such that

$$
\begin{gathered}
\mathbb{P}\left[g\left(\mathbf{y}^{1}\right) \neq i \mid m^{1}=i, m^{2}=k\right] \leq \frac{\epsilon^{\frac{3}{4}}(1-\delta(\nu, n))}{1-\left(1-\epsilon^{\frac{1}{4}}\right)}=\epsilon^{\frac{1}{2}}(1-\delta(\nu, n)), \quad \forall k \in \mathcal{B}_{i}^{1,2}, \\
\left|\mathcal{B}_{i}^{1,2}\right| \geq\left(1-\epsilon^{\frac{1}{4}}\right) 2^{n R_{2}}
\end{gathered}
$$

To summarize, we have obtained that, User 1 is able to use $g(\cdot)$ to decode $\mathbf{y}^{1}=$ $\mathbf{x}_{i}^{1}+h_{12} \mathbf{x}_{k}^{2}+\tilde{\mathbf{z}}_{G}$ correctly as message $i$ with probability at least $1-\epsilon^{\frac{1}{2}}(1-\delta(\nu, n))$. Now consider a single user Gaussian channel, call it $A$, with noise $\tilde{\mathbf{z}}_{G}$ where each component of $\tilde{\mathbf{z}}_{G}$ is independent and normally distributed with mean 0 and standard deviation $(1+2 \nu) \sigma$. Consider a code for channel A with codebook $\mathbb{V}$ given by

$$
\mathbb{V}=\left\{\mathbf{v}_{i k_{i}}=\mathbf{x}_{i}^{1}+h_{12} \mathbf{x}_{k_{i}}^{2}, \forall k_{i} \in \mathcal{B}_{i}^{1,2}, \forall i \in \mathcal{F}^{1}\right\}
$$

with size $\sum_{i \in \mathcal{F}^{1}}\left|\mathcal{B}_{i}^{\mathbf{1}, 2}\right|$. We will next construct a decoding function $\tilde{g}(\cdot): \mathbb{R}^{n} \rightarrow \mathbb{V}$ for channel A by using the interference channel decoding function $g(\cdot)$ as follows:

$$
\tilde{g}\left(\mathbf{y}^{1}\right)=\mathbf{x}_{g\left(\mathbf{y}^{1}\right)}^{1}+h_{12} \mathbf{x}_{h\left(\mathbf{y}^{1}\right)}^{2},
$$

where

$$
h\left(\mathbf{y}^{1}\right)=\arg \min _{\substack{k \in \mathcal{B}^{1,2} \\ g\left(\mathbf{y}^{1}\right)}}\left\|\mathbf{y}^{1}-\left(\mathbf{x}_{g\left(\mathbf{y}^{1}\right)}^{1}+h_{12} \mathbf{x}_{k}^{2}\right)\right\| .
$$

In words, $\tilde{g}(\cdot)$ computes the message index $m^{1}$ by using $g(\cdot)$ and chooses the message index $m^{2}$ by using a minimum distance criterion. Observe that we have the following property that $\tilde{g}(\cdot)$ satisfies:

$$
g\left(\mathbf{y}^{1}\right)=i \Longleftrightarrow \tilde{g}\left(\mathbf{y}^{1}\right) \in \mathbb{V}_{i}, \text { where } \mathbb{V}_{i}=\bigcup_{k^{\prime} \in \mathcal{B}_{i}^{1,2}}\left\{\mathbf{v}_{i k^{\prime}}\right\}
$$

Therefore, using the codebook $\mathbb{V}$ and the decoding function $\tilde{g}(\cdot)$ on channel A, we 
have

$$
\begin{aligned}
\mathbb{P}\left[\tilde{g}\left(\mathbf{v}_{i k}+\tilde{\mathbf{z}}_{G}\right) \in \mathbb{V} \backslash \mathbb{V}_{i} \mid \mathbf{v}_{i k} \text { was sent on } \mathrm{A}\right] & =\mathbb{P}\left[g(\mathbf{y}) \neq i \mid m^{1}=i, m^{2}=k\right] \\
& \leq \epsilon^{\frac{1}{2}}(1-\delta(\nu, n)), \quad(\text { from }(4.81))
\end{aligned}
$$

Furthermore, by taking averages, we have

$$
\frac{1}{|\mathbb{V}|} \sum_{\mathbf{v}_{i k} \in \mathbb{V}} \mathbb{P}\left[\tilde{g}\left(\mathbf{v}_{i k}+\tilde{\mathbf{z}}_{G}\right) \in \mathbb{V} \backslash \mathbb{V}_{i} \mid \mathbf{v}_{i k} \text { was sent on } \mathrm{A}\right] \leq \epsilon^{\frac{1}{2}}(1-\delta(\nu, n))
$$

which from Proposition 36 implies that the minimum distance decoder $g_{0}(\cdot)$ also satisfies

$$
\frac{1}{|\mathbb{V}|} \sum_{\mathbf{v}_{i k} \in \mathbf{V}} \mathbb{P}\left[g_{0}\left(\mathbf{v}_{i k}+\tilde{\mathbf{z}}_{G}\right) \in \mathbb{V} \backslash \mathbb{V}_{i} \mid \mathbf{v}_{i k} \text { was sent on } \mathrm{A}\right] \leq \epsilon^{\frac{1}{2}}(1-\delta(\nu, n))
$$

Applying Proposition 35 with

$$
\begin{gathered}
f=1-\epsilon^{\frac{1}{4}}, N=|\mathbb{V}|, \alpha=\epsilon^{\frac{1}{2}}(1-\delta(\nu, n)), \\
a_{i k}=\mathbb{P}\left[g_{0}\left(\mathbf{v}_{i k}+\tilde{\mathbf{z}}_{G}\right) \in \mathbb{V} \backslash \mathbb{V}_{i} \mid \mathbf{v}_{i k} \text { was sent on } \mathrm{A}\right], \forall \mathbf{v}_{i k} \in \mathbb{V},
\end{gathered}
$$

we obtain that there exists a subset of vectors $\tilde{\mathrm{V}} \subseteq \mathbb{V}$ such that

$$
\begin{gathered}
\mathbb{P}\left[g_{0}\left(\mathbf{v}_{i k}+\tilde{\mathbf{z}}_{G}\right) \in \mathbb{V} \backslash \mathbb{V}_{i} \mid \text { A sent } \mathbf{v}_{i k}\right] \leq \frac{\epsilon^{\frac{1}{2}}(1-\delta(\nu, n))}{1-\left(1-\epsilon^{\frac{1}{4}}\right)}=\epsilon^{\frac{1}{4}}(1-\delta(\nu, n)), \quad \forall \mathbf{v}_{i k} \in \tilde{\mathbb{V}} \\
|\tilde{\mathbb{V}}| \geq\left(1-\epsilon^{\frac{1}{4}}\right)|\mathbb{V}|
\end{gathered}
$$

We now show that (4.84) implies that the quantity $\left\|\mathbf{v}_{i^{\prime} k^{\prime}}-\mathbf{v}_{i k}\right\|$ is lower bounded. 
Consider the probability of incorrectly decoding $\mathbf{v}_{i k}$ as $\mathbf{v}_{\boldsymbol{i}^{\prime} k^{\prime}}$ on channel A, we have

$$
\begin{aligned}
\mathbb{P}\left[\mathbf{v}_{i k} \text { decoded as } \mathbf{v}_{i^{\prime} k^{\prime}}\right] & \geq \mathbb{P}\left[\left\|\mathbf{v}_{i k}-\mathbf{v}_{i^{\prime} k^{\prime}}+\tilde{\mathbf{z}}_{G}\right\| \leq\left\|\tilde{\mathbf{z}}_{G}\right\|\right] \\
& =\mathbb{P}\left[\frac{\left\langle\mathbf{v}_{i k}-\mathbf{v}_{i^{\prime} k^{\prime}}, \tilde{\mathbf{z}}_{G}\right\rangle}{(1+3 \nu) \sigma\left\|\mathbf{v}_{i k}-\mathbf{v}_{i^{\prime} k^{\prime}}\right\|} \geq \frac{\left\|\mathbf{v}_{i k}-\mathbf{v}_{i^{\prime} k^{\prime}}\right\|}{2(1+3 \nu) \sigma}\right] \\
& =1-\Phi\left(\frac{\left\|\mathbf{v}_{i k}-\mathbf{v}_{i^{\prime} k^{\prime}}\right\|}{2(1+3 \nu) \sigma}\right) .
\end{aligned}
$$

Since we know that $\mathbb{P}\left[\mathbf{v}_{i k}\right.$ decoded as $\left.\mathbf{v}_{i^{\prime} k^{\prime}}\right] \leq \epsilon^{\frac{1}{4}}(1-\delta(\nu, n))$, we have

$$
\begin{aligned}
& \epsilon^{\frac{1}{4}}(1-\delta(\nu, n)) \geq 1-\Phi\left(\frac{\left\|\mathbf{v}_{i k}-\mathbf{v}_{i^{\prime} k^{\prime}}\right\|}{2(1+3 \nu) \sigma}\right) \\
\Longrightarrow & \left\|\mathbf{v}_{i k}-\mathbf{v}_{i^{\prime} k^{\prime}}\right\| \geq 2(1+3 \nu) \sigma \Phi^{-1}\left(1-\epsilon^{\frac{1}{4}}(1-\delta(\nu, n))\right) \\
\Longrightarrow & \left\|\mathbf{v}_{i k}-\mathbf{v}_{i^{\prime} k^{\prime}}\right\| \geq 2 \sqrt{n}(1+3 \nu) \zeta,
\end{aligned}
$$

which implies that the codewords satisfy the constraints (4.52).

We next show that remaining constraints are also satisfied. Let $\tilde{\mathbf{z}}_{U}$ be uniformly distributed in $\mathcal{S}^{n}\left(\sigma_{1} \sqrt{n}\right)$, where

$$
\sigma_{1}=(1+2 \nu) \sigma<(1+3 \nu) \sigma
$$

and let $\tilde{\mathbf{z}}_{D}$ be a discrete uniform distribution given by

$$
\mathbb{P}\left[\tilde{\mathbf{z}}_{D}=\mathbf{z}_{t}\right]=\frac{1}{T}, \forall t=1, \ldots, T
$$

Consider a $\mathbf{z}_{t}$ and an $\mathbf{s} \in \mathcal{S}^{n}\left(\sigma_{1} \sqrt{n}\right)$ such that $\mathbf{s}$ is in the Voronoi region $\mathcal{V}\left(\mathbf{u}_{t}\right)$ of $\mathbf{u}_{t}=\sigma_{1} \mathbf{z}_{t} /(1+\nu) \sigma$, that is, $\mathbf{s} \in \mathcal{V}\left(\mathbf{u}_{t}\right)$. Applying Proposition 29 to $\mathcal{A}=$ $\left\{\mathbf{u}_{1}, \ldots, \mathbf{u}_{T}\right\}, N=T, \Lambda=\sigma_{1}$, we obtain

$$
\left\|\mathbf{s}-\mathbf{u}_{t}\right\| \leq \theta^{\prime} \sqrt{n}, \text { for } \mathbf{u}_{t}=\frac{1+2 \nu}{1+\nu} \mathbf{z}_{t}
$$


where $2 \theta^{\prime} \sqrt{n} \leq \frac{\nu}{1+\nu}\left\|\mathbf{v}_{i k}-\mathbf{v}_{i^{\prime} k^{\prime}}\right\|$ from (4.39). Then, if $\left\|\mathbf{v}_{i k}-\mathbf{v}_{i^{\prime} k^{\prime}}+\mathbf{z}_{t}\right\| \leq\left\|\mathbf{z}_{t}\right\|$,

$$
\begin{aligned}
2\left\langle\mathbf{v}_{i^{\prime} k^{\prime}}-\mathbf{v}_{i k}, \mathbf{s}\right\rangle & =2\left\langle\mathbf{v}_{i^{\prime} k^{\prime}}-\mathbf{v}_{i k},\left(\mathbf{s}-\mathbf{u}_{t}\right)+\mathbf{u}_{t}\right\rangle \\
& \geq 2\left\langle\mathbf{v}_{i^{\prime} k^{\prime}}-\mathbf{v}_{i k}, \mathbf{u}_{t}\right\rangle-2 \cdot\left\|\mathbf{v}_{i^{\prime} k^{\prime}}-\mathbf{v}_{i k}\right\| \cdot\left\|\mathbf{s}-\mathbf{u}_{t}\right\| \quad \text { (Cauchy-Schwartz) } \\
& \geq \frac{1+2 \nu}{1+\nu}\left\|\mathbf{v}_{i^{\prime} k^{\prime}}-\mathbf{v}_{i k}\right\|^{2}-2 \cdot\left\|\mathbf{v}_{i^{\prime} k^{\prime}}-\mathbf{v}_{i k}\right\| \cdot \theta^{\prime} \sqrt{n} \\
& =\left\|\mathbf{v}_{i k}-\mathbf{v}_{i^{\prime} k^{\prime}}\right\| \cdot\left\{\frac{1+2 \nu}{1+\nu}\left\|\mathbf{v}_{i k}-\mathbf{v}_{i^{\prime} k^{\prime}}\right\|-2 \theta^{\prime} \sqrt{n}\right\} \\
& \geq\left\|\mathbf{v}_{i k}-\mathbf{v}_{i^{\prime} k^{\prime}}\right\| \cdot\left\{\frac{1+2 \nu}{1+\nu}\left\|\mathbf{v}_{i k}-\mathbf{v}_{i^{\prime} k^{\prime}}\right\|-\frac{\nu}{1+\nu} \cdot\left\|\mathbf{v}_{i k}-\mathbf{v}_{i^{\prime} k^{\prime}}\right\|\right\} \\
& =\left\|\mathbf{v}_{i k}-\mathbf{v}_{i^{\prime} k^{\prime}}\right\|^{2} \cdot\left\{\frac{1+2 \nu}{1+\nu}-\frac{\nu}{1+\nu}\right\}=\left\|\mathbf{v}_{i k}-\mathbf{v}_{i^{\prime} k^{\prime}}\right\|^{2} .
\end{aligned}
$$

That is,

$$
\text { if }\left\|\mathbf{v}_{i k}-\mathbf{v}_{i^{\prime} k^{\prime}}+\mathbf{z}_{t}\right\| \leq\left\|\mathbf{z}_{t}\right\| \text {, then }\left\|\mathbf{v}_{i k}-\mathbf{v}_{i^{\prime} k^{\prime}}+\mathbf{s}\right\| \leq\|\mathbf{s}\| \forall \mathbf{s} \in \mathcal{V}\left(\mathbf{u}_{t}\right)
$$

Next, we have $\mathbb{P}\left[\mathscr{E}_{i k}^{1}\left[\tilde{\boldsymbol{z}}_{U}\right]\right]$ given by

$$
\mathbb{P}\left[\mathscr{E}_{i k}^{1}\left[\tilde{\boldsymbol{z}}_{U}\right]\right]=\sum_{t=1}^{T} \mathbb{P}\left[\mathscr{E}_{i k}^{1}\left[\overline{\boldsymbol{z}}_{U}\right] \mid \overline{\boldsymbol{z}}_{U} \in \mathcal{V}\left(\mathbf{u}_{t}\right)\right] \cdot \mathbb{P}\left[\tilde{\boldsymbol{z}}_{U} \in \mathcal{V}\left(\mathbf{u}_{t}\right)\right]
$$

Since the set of vectors $\left\{\mathbf{z}_{t}\right\}$ form a Voronoi tessellation, the vectors $\left\{\mathbf{u}_{t}\right\}$ also form a Voronoi tessellation on $\mathcal{S}^{n}\left(\sigma_{1} \sqrt{n}\right)$. Therefore, the Voronoi regions of the points $\mathbf{u}_{t}$ are identical with the same area. Consequently,

$$
\mathbb{P}\left[\tilde{z}_{U} \in \mathcal{V}\left(\mathbf{u}_{t}\right)\right]=\frac{1}{T}, \forall t=1, \ldots, T
$$

Moreover, from (4.87), we have

$$
\mathbb{P}\left[\mathscr{E}_{i}\left[\tilde{\boldsymbol{z}}_{U}\right] \mid \tilde{\boldsymbol{z}}_{U} \in \mathcal{V}\left(\mathbf{u}_{t}\right)\right] \geq 1\left\{\left\|\mathbf{x}_{i}-\mathbf{x}_{k}+\mathbf{z}_{t}\right\| \leq\left\|\mathbf{z}_{t}\right\|\right\}
$$


Substituting (4.89) and (4.90) in (4.88), we have

$$
\mathbb{P}\left[\mathscr{E}_{i k}^{\mathbf{1}}\left[\tilde{\boldsymbol{z}}_{U}\right]\right] \geq \frac{1}{T} \sum_{t=1}^{T} \mathbf{1}\left\{\left\|\mathbf{x}_{i}-\mathbf{x}_{k}+\mathbf{z}_{t}\right\| \leq\left\|\mathbf{z}_{t}\right\|\right\}
$$

From Proposition 38 we have,

$$
\mathbb{P}\left[\mathscr{E}_{i k}^{1}\left[\tilde{\boldsymbol{z}}_{U}\right]\right] \leq \frac{\mathbb{P}\left[\mathscr{E}_{i k}^{1}\left[\tilde{\boldsymbol{z}}_{G}\right]\right]}{1-\delta(\nu, n)} \leq \frac{\epsilon^{\frac{1}{4}}(1-\delta(\nu, n))}{1-\delta(\nu, n)}=\epsilon^{\frac{1}{4}}
$$

Therefore, we must have that

$$
\frac{1}{T} \sum_{t=1}^{T} 1\left\{\left\|\mathbf{v}_{i k}-\mathbf{v}_{i^{\prime} k^{\prime}}+\mathbf{z}_{\ell}\right\| \leq\left\|\mathbf{z}_{l}\right\|\right\} \leq \mathbb{P}\left[\mathscr{E}_{i k}^{1}\left[\tilde{\boldsymbol{z}}_{U}\right]\right] \leq \epsilon^{\frac{1}{4}}
$$

This implies that the codewords satisfy the constraints (4.51). This observation along with the repetition of a similar argument from the point of view of User 2 concludes the proof.

\subsubsection{Remarks on the Optimality of the Decoder (4.48)}

The key idea between our characterization of the capacity region of the two user Gaussian interference channel has been the use of the decoder (4.48). The origin of this decoder can be intuited from the proof of Part (b) of Theorem 40. Recall that the proof involved a construction of a "desirable" code for a single user Gaussian channel, from an arbitrary "desirable" code of a two-user Gaussian channel. Given that we have a complete understanding of an optimal decoder for a single user channel, this reduction was essential to the proof. Moreover, this reduction allowed us to identify the constraints that the codewords of an arbitrary "desirable" code for an interference channel satisfy. These constraints in turn lead to the optimality of the decoder (4.48) for the two-user interference channel. 


\begin{tabular}{|c|c|c|}
\hline Noise & Typical Set & Optimization Problem \\
\hline \hline Gaussian (independent) & Ball & $\begin{array}{l}\text { Rank minimization with } \\
\text { semidefinite constraints }\end{array}$ \\
\hline Gaussian (correlated) & Ellipsoid & $\begin{array}{l}\text { Rank minimization with } \\
\text { semidefinite constraints }\end{array}$ \\
\hline Exponential & Polyhedron & $\begin{array}{l}\text { Binary mixed linear } \\
\text { optimization problem }\end{array}$ \\
\hline Uniform & Polyhedron & $\begin{array}{l}\text { Binary mixed linear } \\
\text { optimization problem }\end{array}$ \\
\hline Binary symmetric noise & Polyhedron & $\begin{array}{l}\text { Binary optimization } \\
\text { problem }\end{array}$ \\
\hline
\end{tabular}

Table 4.1: Dependence of the nature of the optimization problem with noise distribution.

\subsection{Channels with Exponential Noise}

Our goal in this section is to shed light on how the optimization problem we solve is linked to the specific probabilistic assumptions we make on the noise of the channel. In previous sections, we have seen that if the noise is Gaussian, the underlying optimization problem becomes a rank minimization problem with semidefinite constraints. In this section, we show that when the noise is exponentially distributed, then the underlying problem is a binary mixed linear optimization problem for which there are successful commercial solvers that can solve problem instances of very high dimensions. To amplify this point, we present in Table 1 the nature of the underlying optimization problem as the noise distribution varies.

\subsubsection{Single User Exponential Channel - Capacity Charac- terization and Optimal Coding}

Recall that the typical set for an exponential distribution with parameter $\lambda$ is given by

$$
\mathcal{U}_{\epsilon}^{\operatorname{Exp}}=\left\{\left(z_{1}, \ldots, z_{n}\right) \mid \frac{n}{\lambda}-\sqrt{n} \frac{\Gamma_{\epsilon}^{\operatorname{Exp}}}{\lambda} \leq \sum_{j=1}^{n} z_{j} \leq \frac{n}{\lambda}+\sqrt{n} \frac{\Gamma_{\epsilon}^{\operatorname{Exp}}}{\lambda}\right\},
$$

where $\Gamma_{\epsilon}^{\operatorname{Exp}}$ is chosen such that $\mathbb{P}\left[\tilde{\mathbf{z}} \in \mathcal{U}_{\epsilon}^{\operatorname{Exp}}\right]=1-\epsilon$ when each component of $\tilde{\mathbf{z}}$ is distributed exponentially with parameter $\lambda$. We next obtain the maximum likelihood 
decoder for the exponential channel.

Proposition 41. The maximum likelihood decoder for the exponential channel is given by

$$
g_{0}^{E x p}(\mathbf{y})=\arg \min _{i \in \mathcal{B}(\mathbf{y})} \sum_{j=1}^{n}\left(y_{j}-x_{i j}\right)
$$

where $\mathcal{B}(\mathbf{y})=\left\{i \in \mathcal{B} \mid y_{j} \geq x_{i j}, \forall j=1, \ldots, n\right\}$

Proof. Suppose a vector $\mathbf{y}$ is received. Since exponential noise is non-negative, any candidate codeword $\mathbf{x}_{i}$ that we consider must satisfy

$$
y_{j}-x_{i j} \geq 0, \forall j=1, \ldots, n
$$

Among all such codewords, the maximum likelihood decoder is given by

$$
\begin{aligned}
g_{0}^{\operatorname{Exp}}(\mathbf{y}) & =\arg \max _{i \in \mathcal{B}(\mathbf{y})} \prod_{j=1}^{n} \lambda \exp \left(-\lambda\left(y_{j}-x_{i j}\right)\right) \\
& =\arg \min _{i \in \mathcal{B}(\mathbf{y})} \sum_{j=1}^{n}\left(y_{j}-x_{i j}\right)
\end{aligned}
$$

Motivated by this, we next present a robust optimization problem (see Eqs. (4.94)(4.99)) that allows us to characterize the capacity region of a single user exponential channel. In particular, given inputs $n, R, \lambda, P, \epsilon, \nu$, we calculate the "derivative" quantities $\gamma_{\epsilon}^{\mathrm{Exp}}, T^{\mathrm{Exp}}$ and $M_{0}^{\mathrm{Exp}}$ as follows:

(a) The parameter $\gamma_{\epsilon}^{\mathrm{Exp}}$, which we choose so that

$$
\mathbb{P}\left[\sum_{i=1}^{n} \tilde{z}_{i} \leq \gamma_{\epsilon}\right] \geq 1-\epsilon
$$

where $\tilde{\mathbf{z}} \sim \operatorname{Exponential}(\boldsymbol{\lambda})$; 
(b) The parameter $T^{\text {Exp }}$ given by

$$
T=\left(\frac{1+2 \nu}{\zeta^{\operatorname{Exp}} \nu} \cdot \frac{\gamma_{\epsilon}^{\operatorname{Exp}}}{\sqrt{n}}\right)^{n}, \text { with } \zeta^{\operatorname{Exp}}=\frac{1}{\lambda \sqrt{n}} \cdot \Psi^{-1}(1-\epsilon)
$$

where $\Psi(\cdot)$ is the cdf of the exponential distribution;

(c) The parameter $M_{0}^{\text {Exp }}=(1+2 \nu) \cdot \gamma_{\epsilon}^{\text {Exp }}$.

In addition, generate a Voronoi tesselation $\left\{\mathbf{z}_{1}^{\text {Exp }}, \mathbf{z}_{2}^{\operatorname{Exp}}, \ldots, \mathbf{z}_{T}^{\text {Exp }}\right\}$ of the simplex

$$
\mathcal{P}_{\epsilon}=\left\{\left(z_{1}, \ldots, z_{n}\right) \mid \sum_{j=1}^{n} z_{j}=\frac{n}{\lambda}+\sqrt{n} \frac{\Gamma_{\epsilon}^{\operatorname{Exp}}}{\lambda}\right\}
$$

We next use the decision variables $\mathbf{x}_{i}, \forall i=1, \ldots, 2^{n R}$ and $v_{i t}, \forall i=1, \ldots, 2^{n R}, \forall t=$ $1, \ldots, T$, where

1. The variables $\mathbf{x}_{i}$ represent the codewords.

2. The variables $v_{i t}$ represent binary decision variables that are chosen in a way to constrain the probability of error. When $v_{i t}=1$, the set of decoding constraints (4.96) are satisfied for codeword $\mathbf{x}_{i}$ with noise vector $\mathbf{z}_{t}^{\text {Exp }}$.

We construct the following mixed-integer optimization problem:

$$
\begin{array}{cc}
\sum_{i, k, t} v_{i k t} & \\
\sum_{j=1}^{n} x_{i j} \leq n P, & \forall i=1, \ldots, 2^{n R}, \\
\sum_{j=1}^{n} x_{i j}+\left(2-v_{i t}-v_{i k t}\right) M_{0} \geq \sum_{j=1}^{n} x_{k j}, & \forall t, \forall i, k \neq i, \\
x_{i j}+z_{t j}^{\operatorname{Exp}} \geq x_{k j}-M_{0}\left(1-v_{i k t}\right), & \forall i, k, j, t, \\
\sum_{t=1}^{T} v_{i t} \geq(1-\epsilon) T, & \forall i, \\
v_{i t}, v_{i k t} \in\{0,1\}, & \forall i, k, t,
\end{array}
$$

Constraints (4.95) impose power constraints on the codewords. Constraints (4.96), 
(4.98) ensure that each codeword $\mathbf{x}_{i}$ is decoded correctly for at least $(1-\epsilon) T$ of the noise vectors.

We next present Algorithm 5, without proof, that characterizes the capacity region of this channel.

Algorithm 5 Capacity Characterization and Optimal Coding for the Single User Exponential Channel.

Input : $R, P, \lambda, n, \nu, \epsilon$.

Output : Codewords $\left\{\mathbf{x}_{i}\right\}_{i=1}^{2^{n R}}$, and auxiliary binary variables $\left\{v_{i t}, v_{i k t}\right\}$.

\section{Algorithm:}

1. Solve the binary mixed linear optimization problem (4.94) to compute the codewords $\left\{\mathbf{x}_{i}\right\}_{i=1}^{2^{n R}}$, and auxiliary binary variables $\left\{v_{i t}, v_{i k t}\right\}$.

2. If the problem is feasible, then $R \in \mathcal{R}_{n}^{\mathrm{Exp}}[P, \lambda, 2 \epsilon]$, that is, $R$ can be achieved on an exponential channel using the codewords $\left\{\mathrm{x}_{i}\right\}_{i=1}^{2^{n R}}$ and the decoding function $g_{0}^{\text {Exp }}(\cdot)$, with a decoding error probability of at most $\epsilon$.

3. If the problem is infeasible, then $R \notin \mathcal{R}_{n}^{\operatorname{Exp}}\left[P, \frac{\lambda}{1+2 \nu}, \epsilon\right]$.

\subsubsection{Two-User Exponential Interference Channel - Capacity Characterization and Optimal Coding}

In this section, we present an algorithmic way to characterize the capacity region of a two-user exponential channel. We borrow notation from Section 4.4, and present the algorithms that characterize the capacity region. Just as in the case of a Gaussian channel, we first identify a decoding function that will suffice to construct optimal codes. In this case, the optimal decoder is given by

$$
i_{1}^{*}(\mathbf{y})=\arg \max _{i \in \mathcal{B}^{1}}\left[\max _{k \in \mathcal{B}_{i}^{2}(\mathbf{y})} \sum_{j=1}^{n}\left(x_{i j}^{1}+h_{12} x_{k j}^{2}\right)\right],
$$


where $\mathcal{B}_{i}^{2}(\mathbf{y})=\left\{k \in \mathcal{B}^{2} \mid y_{j} \geq x_{i j}^{1}+h_{12} x_{k j}^{2}, \forall j=1, \ldots, n\right\}$. Motivated by this, we next present the optimization problem that characterizes the capacity region of an exponential interference channel in Algorithm 6.

Just as in the case of the Gaussian channel, it can be shown that Algorithm 6, correctly characterizes the capacity of the exponential interference channel.

\subsection{The Multi-access Channel and the Multi-cast Channel}

Multi-access and multi-cast channels special cases of the interference channel where either the receivers cooperate or the senders cooperate, respectively.

\subsubsection{Capacity Region of a Gaussian Multi-access channel (MAC)}

This is a channel in which two (or more) senders send information to a common receiver. A common example of this channel is a satellite receiver with many independent ground stations, or a set of cell phones communicating with a base station. As in interference channels, we see that the senders must contend not only with the receiver noise but with interference from each other as well. Sender $k$ chooses a message $m^{k}=i_{k} \in \mathcal{B}^{k}$ and transmits $\mathbf{x}_{i_{k}}^{k}$ over the channel while satisfying

$$
\left\|\mathrm{x}_{i_{k}}^{k}\right\|^{2} \leq P_{k}
$$

Other senders do likewise. The receiver receives the combined signal

$$
\mathbf{y}=\sum_{k=1}^{K} \mathbf{x}_{i_{k}}^{k}+\tilde{\mathbf{z}}
$$

where $\tilde{\mathbf{z}}$ is the Gaussian noise. The receiver is required to decode all the $K$ messages from the senders. In this section, for the sake of convenience, we let $m^{k}=i$ denote 
Algorithm 6 Encoding algorithm for two-user exponential interference channel.

Input: $n, R_{1}, R_{2}, \lambda, P_{1}, P_{2}, \epsilon, \nu$.

Output: Codewords $\mathrm{x}_{i}^{1}, \mathbf{x}_{k}^{2}$, and binary variables $v_{i k t}^{1}, v_{i k i^{\prime} k^{\prime} t}^{1}, v_{i k t}^{2}, v_{i k i^{\prime} k^{\prime} t}^{2}$.

\section{Algorithm:}

1. Solve the binary mixed linear optimization problem:

$$
\begin{aligned}
& \max \\
& \sum_{i, k, t} v_{i k i^{\prime} k^{\prime} t}^{1}+\sum_{i, k, t} v_{i k i^{\prime} k^{\prime} t}^{2} \\
& \sum_{j=1}^{n} x_{i j}^{1} \leq n P_{1} \\
& \sum_{j=1}^{n} x_{i j}^{1}+h_{12} \sum_{j=1}^{n} x_{k j}^{2}+\left(2-v_{i k t}^{1}-v_{i k i^{\prime} k^{\prime} t}^{1}\right) M_{0}^{\text {Exp }} \\
& \geq \sum_{j=1}^{n} x_{i^{\prime} j}^{1}+h_{12} \sum_{j=1}^{n} x_{k^{\prime} j}^{2} \\
& x_{i j}^{1}+h_{12} x_{k j}^{2}+z_{t j}^{\operatorname{Exp}} \geq \\
& x_{i^{\prime} j}^{1}+h_{12} x_{k^{\prime} j}^{2}-M_{0}^{\mathrm{Exp}}\left(1-v_{i k i^{\prime} k^{\prime} t}^{1}\right), \quad \forall i, k, j, t, \\
& \sum_{t=1}^{T} v_{i k t}^{1} \geq(1-\epsilon) T, \quad \forall i \\
& \sum_{j=1}^{n} x_{i j}^{2} \leq n P_{2}, \quad \forall i \\
& \sum_{j=1}^{n} x_{i j}^{2}+h_{21} \sum_{j=1}^{n} x_{k j}^{1}+\left(2-v_{i k t}^{2}-v_{i k i^{\prime} k^{\prime} t}^{2}\right) M_{0}^{\text {Exp }} \\
& \geq \sum_{j=1}^{n} x_{i^{\prime} j}^{2}+h_{21} \sum_{j=1}^{n} x_{k^{\prime} j}^{1} \\
& x_{i j}^{2}+h_{21} x_{k j}^{1}+z_{t j}^{\operatorname{Exp}} \geq \\
& x_{i^{\prime} j}^{2}+h_{21} x_{k^{\prime} j}^{1}-M_{0}^{\mathrm{Exp}}\left(1-v_{i k i^{\prime} k^{\prime} t}^{2}\right), \quad \forall i, k, j, t, \\
& \sum_{t=1}^{T} v_{i k t}^{2} \geq(1-\epsilon) T \\
& v_{i k t}^{1}, v_{i k i^{\prime} k^{\prime} t}^{1}, v_{i k t}^{2}, v_{i k i^{\prime} k^{\prime} t}^{2} \in\{0,1\}, \quad \forall i, k, t .
\end{aligned}
$$

2. If the problem defined by constraints (4.101)-(4.111) is feasible, then $\left(R_{1}, R_{2}\right)$ is achievable and the resulting codewords can be used to transmit messages. 
that sender $k$ transmits message $i \in \mathcal{B}^{k}$.

In what follows, we consider the special case of a two-user multi-access channel. The channel coding problem for such a channel refers to the problem of constructing a code $\mathcal{C}^{\mathrm{MAC}}[\cdot]$ with inputs $n, R_{1}, R_{2}, \sigma, P_{1}, P_{2}, \epsilon$.

The outputs of $\mathcal{C}^{\mathrm{MAC}}\left[n, P_{1}, P_{2}, \sigma, R_{1}, R_{2}, \epsilon\right]$ are:

(a) The codebooks $\mathcal{B}^{1}=\left\{\mathrm{x}_{i}^{1}\right\}_{i=1}^{2^{n R_{1}}}, \mathcal{B}^{2}=\left\{\mathrm{x}_{k}^{2}\right\}_{k=1}^{2^{n R_{2}}}$;

(b) The decoding function $h: \mathbb{R}^{n} \rightarrow \mathcal{B}^{1} \times \mathcal{B}^{2}$ that map each received codeword $\mathbf{y}$ to a pair of codewords in $\mathcal{B}^{1}$ and $\mathcal{B}^{2}$, so that the average probability of error satisfies

$$
\mathbb{P}\left[h(\mathbf{y}) \neq(i, k) \mid m^{1}=i, m^{2}=k\right] \leq \epsilon
$$

In addition, we define the capacity region of a two-user multi-cast channel

$$
\mathcal{R}_{n}^{\mathrm{MAC}}\left[P_{1}, P_{2}, \sigma, \epsilon\right]
$$

as the set of all rates $\left(R_{1}, R_{2}\right)$ such that there exists a code $\mathcal{C}^{\mathrm{MAC}}\left[n, P_{1}, P_{2}, \sigma, R_{1}, R_{2}, \epsilon\right]$.

\section{Capacity Characterization and Optimal Coding}

In this section, we present the algorithm (Algorithm 4) which we use to characterize the capacity region while simultaneously constructing the optimal code. The algorithm consists of two parts: (a) the Encoding Algorithm (Algorithm 7), and (b) the Decoding Algorithm (Algorithm 8). A key ingredient in our construction of this algorithm is that the maximum likelihood decoder is the minimum distance decoder for this problem. In particular, we use the following decoder

$$
\left(i^{*}, k^{*}\right)=\arg \operatorname{minmin}_{i \in \mathcal{B}^{1}}\left\|\mathbf{m} \in \mathcal{B}^{2}-\left(\mathbf{x}_{i}^{1}+\mathbf{x}_{k}^{2}\right)\right\|
$$

Algorithm 4 is based on a feasibility problem with the following decision variables: 
Algorithm 7 Encoding Algorithm for two-user Multi-access Channel Input: $n, R_{1}, R_{2}, \sigma, P_{1}, P_{2}, \epsilon, \nu$.

Output : Codewords $\mathbf{x}_{i}^{1}, \mathbf{x}_{k}^{2}$, and binary variables $v_{i k},\left\{v_{i k t}\right\}_{t=1}^{T}$.

Algorithm :

1. Calculate the quantities $T, M_{0}$, and the set of equidistant vectors $\mathcal{Z}=\left\{\mathbf{z}_{t}\right\}_{t=1}^{T}$.

2. Check the feasibility of the constraints

$$
\begin{array}{cl}
\left\|\mathbf{x}_{i}^{1}\right\|^{2} \leq n P_{1} & \forall i=1, \ldots, 2^{n R_{1}}, \\
\left\|\mathbf{x}_{k}^{2}\right\|^{2} \leq n P_{2} & \forall k=1, \ldots, 2^{n R_{2}}, \\
\left\|\left(\mathbf{x}_{i}^{1}+\mathbf{x}_{k}^{2}\right)-\left(\mathbf{x}_{i^{\prime}}^{1}+\mathbf{x}_{k^{\prime}}^{2}\right)+\mathbf{z}_{t}\right\| & \\
+\left(1-v_{i k t}\right) M_{0} \geq\left\|\mathbf{z}_{t}\right\|, & \forall \mathbf{z}_{t} \in\left\{\mathbf{z}_{1}, \ldots, \mathbf{z}_{T}\right\} \\
\left\|\left(\mathbf{x}_{i}^{1}+\mathbf{x}_{k}^{2}\right)-\left(\mathbf{x}_{i^{\prime}}^{1}+\mathbf{x}_{k^{\prime}}^{2}\right)\right\| \geq 2 \sigma \Phi^{-1}\left(1-\epsilon^{\frac{1}{2}}\right), & \forall i, k \\
\sum_{t=1}^{T} v_{i k t} \geq\left(1-\epsilon^{\frac{1}{2}}\right) T, & \forall i, k \\
v_{i k t} \in\{0,1\}, & \forall i, k t
\end{array}
$$

3. If feasible,then $\left(R_{1}, R_{2}\right)$ is achievable and the resulting codewords can be used to transmit messages.

(a) Variables $\left\{\mathbf{x}_{i}^{1}\right\}_{i=1}^{2^{n R_{1}}},\left\{\mathbf{x}_{k}^{2}\right\}_{k=1}^{2^{n R_{2}}}$ that represent the codewords for Users 1 and 2, respectively;

(b) Binary variables $\left\{v_{i k t}\right\}_{t=1}^{T}$ are auxiliary variables which allow us to constrain the probability of incorrect decoding for codewords identified by $v_{i k}$. In particular, for codewords $i, k$ with $v_{i k}=1$

$$
v_{i k t}= \begin{cases}1, & \text { if }\left\|\left(\mathbf{x}_{i}^{1}+\mathbf{x}_{k}^{2}\right)-\left(\mathbf{x}_{i^{\prime}}^{1}+\mathbf{x}_{k^{\prime}}^{2}\right)+\mathbf{z}_{t}\right\| \geq\left\|\mathbf{z}_{t}\right\|, \forall i^{\prime}, k^{\prime} \\ 0, & \text { otherwise. }\end{cases}
$$

As before, we use Proposition 27 to reformulate the feasibility problem into an equivalent rank-constrained semidefinite optimization problem. We then use the resulting rank-constrained optimization problem in the following algorithm that, as we show in Theorem 42, correctly characterizes the capacity region: 
Algorithm 8 Decoding Algorithm for two-user Multi-access Channel

Input : Received codeword $\mathbf{y}$.

Output: Messages $i_{1}^{*}, i_{2}^{*}$.

Algorithm :

1. Solve

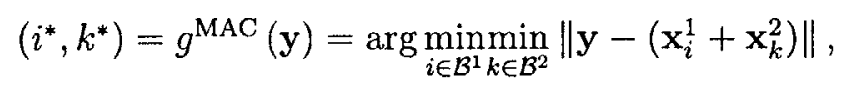

$\overline{\text { Algorithm } 9 \text { Capacity Characterization and Optimal Coding for the Two-User }}$ Multi-access Channel

Input : $R_{1}, R_{2}, P_{1}, P_{2}, \sigma, n, \epsilon, \nu$.

Output : Rank $r^{*}$, codewords $\left\{\mathbf{x}_{i}^{1}\right\}_{i=1}^{2^{n R_{1}}},\left\{\mathbf{x}_{k}^{2}\right\}_{k=1}^{2^{n R_{2}}}$, and auxiliary binary variables $\left\{v_{i k t}\right\}_{t=1}^{T}$.

\section{Algorithm :}

1. Solve the rank minimization semidefinite optimization problem to compute $r^{*}$.

2. If $r^{*}=1$, then $\left(R_{1}, R_{2}\right)$ is achievable using the codebooks $\mathcal{B}^{1}=\left\{\mathbf{x}_{i}^{1}\right\}_{i=1}^{2^{n R_{1}}}$, $\mathcal{B}^{2}=\left\{\mathrm{x}_{k}^{2}\right\}_{k=1}^{2^{n R_{2}}}$ and the decoding functions (4.113), achieving a decoding error probability of $\epsilon$. That is,

$$
\text { If } r^{*}=1 \text {, then }\left(R_{1}, R_{2}\right) \in \mathcal{R}_{n}^{\mathrm{MAC}}\left[P_{1}, P_{2}, \sigma, \epsilon\right] \text {. }
$$

3. If $r^{*} \geq 2$, then we can conclude using Theorem 42 that, $\left(R_{1}, R_{2}\right)$ cannot be achieved on a Gaussian multi-access channel with noise standard deviation (1+ $2 \nu) \sigma$ with probability of error less than or equal to $\epsilon$. That is,

$$
\text { If } r^{*} \geq 2, \text { then }\left(R_{1}, R_{2}\right) \notin \mathcal{R}_{n}^{\mathrm{MAC}}\left[P_{1}, P_{2},(1+2 \nu) \sigma, \epsilon\right]
$$

Theorem 42. Let $r^{*},\left\{\mathbf{x}_{i}^{1}\right\}_{i=1}^{2^{n R_{1}}},\left\{\mathrm{x}_{k}^{2}\right\}_{k=1}^{2^{n R_{2}}}$ be an optimal solution of the rank-constrained SDP formulation of (4.114-4.119).

(a) If $r^{*}=1$, then $\left(R_{1}, R_{2}\right)$ is achievable using the codebooks $\mathcal{B}^{1}=\left\{\mathbf{x}_{i}^{1}\right\}_{i=1}^{2^{n R_{1}}}$, 
$\mathcal{B}^{2}=\left\{\mathrm{x}_{k}^{2}\right\}_{k=1}^{2^{n R_{2}}}$ and the decoding functions (4.113), achieving a decoding error probability of $\epsilon$. That is,

$$
\text { If } r^{*}=1, \text { then }\left(R_{1}, R_{2}\right) \in \mathcal{R}_{n}^{M A C}\left[P_{1}, P_{2}, \sigma, \epsilon\right] \text {. }
$$

(b) If $r^{*} \geq 2$, then $\left(R_{1}, R_{2}\right)$ cannot be achieved on a Gaussian multi-access channel with noise standard deviation $(1+2 \nu) \sigma$ with probability of error less than or equal to $\epsilon$. That is,

$$
\text { If } r^{*} \geq 2, \text { then }\left(R_{1}, R_{2}\right) \notin \mathcal{R}_{n}^{M A C}\left[P_{1}, P_{2},(1+2 \nu) \sigma, \epsilon\right]
$$

Proof. The proof follows from the fact that a multi-access channel is a special case of an interference channel.

We also provide an alternate proof of the following well known result on multiaccess channels.

Theorem 43. Consider a multi-access channel with $K$ transmitters and one receiver. The achievable rate region $\mathcal{R}_{n}^{M A C}\left[P_{1}, P_{2}, \ldots, P_{K}, \sigma, \epsilon\right]$ is given by

$$
\begin{aligned}
& \mathcal{R}_{n}^{\text {MAC }}\left[P_{1}, P_{2}, \ldots, P_{K}, \sigma, \epsilon\right]= \\
& \left.\left(R_{1}, \ldots, R_{K}\right) \mid \begin{array}{rc}
R_{i} \leq \frac{1}{2} \log \left(1+\frac{P_{i}}{\sigma^{2}}\right), & \forall i, \\
R_{i}+R_{j} \leq \frac{1}{2} \log \left(1+\frac{P_{i}+P_{j}}{\sigma^{2}}\right), & \forall i, j, \\
R_{i}+R_{j}+R_{k} \leq \frac{1}{2} \log \left(1+\frac{P_{i}+P_{j}+P_{k}}{\sigma^{2}}\right), & \forall i, j, k \\
\vdots & \vdots \\
R_{1}+R_{2}+\ldots+R_{K} \leq \frac{1}{2} \log \left(1+\frac{P_{i}+\ldots+P_{K}}{\sigma^{2}}\right), &
\end{array}\right\}
\end{aligned}
$$

where $\mathcal{R}_{n}[P, \sigma, \epsilon]$ is the capacity region of user with power constraint $P$ on a single user Gaussian channel with noise standard deviation $\sigma$.

Proof. Suppose $\left(R_{1}, \ldots, R_{K}\right) \in \mathcal{R}_{n}^{\mathrm{MAC}}\left[P_{1}, P_{2}, \ldots, P_{K}, \sigma, \epsilon\right]$, then there exist code- 
books

$$
\mathcal{B}^{1}, \ldots, \mathcal{B}^{K}
$$

that satisfy the constraints (4.114)-(4.119). Using these codewords we construct construct $2^{n\left(R_{1}+\ldots, R_{K}\right)}$ distinct codewords for a single user Gaussian channel by simply adding up individual codewords of the MAC. In particular a codeword is obtained as follows

$$
\mathrm{x}_{i_{1} i_{2} \ldots i_{K}}=\sum_{k=1}^{K} \mathbf{x}_{i_{k}}^{k} .
$$

It is easy to see that the power constraints are satisfied

$$
\begin{aligned}
\left\|\mathbf{x}_{i_{1} i_{2} \ldots i_{K}}\right\| & =\left\|\sum_{k=1}^{K} \mathbf{x}_{i_{k}}^{k}\right\| \\
& \leq \sum_{k=1}^{K}\left\|\mathbf{x}_{i_{k}}^{k}\right\| \\
& =P_{1}+\ldots+P_{K} .
\end{aligned}
$$

The decoding constraints are also trivially satisfied, and in fact the decoding constraints in (4.116) and (4.96) are identical to each other. Thus we construct a code for the single user Gaussian Channel from the code of a MAC, and thus the constraint

$$
R_{1}+R_{2}+\ldots+R_{K} \in \mathcal{R}_{n}\left[P_{i}+P_{j}+\ldots+P_{k}, \sigma, \epsilon\right]
$$

holds true. The fact that the remaining constraints also hold is proved exactly in the same way. This concludes the proof.

\subsubsection{Multi-cast Channel}

The multi-cast channel is a communication channel in which there is one sender and two or more receivers. The basic problem is to find the set of simultaneously achievable rates for communication in a broadcast channel. Here we consider the case of two receivers. Assume that we have a sender of power $P$ and two distant receivers. To encode the messages, the transmitter first picks a parameter $\alpha \in[0,1]$ 
to generate two codebooks, one with power $\alpha P$ at rate $R_{1}$, and another with power $(1-\alpha) P$ at rate $R_{2}$, where $\left\{R_{1}, R_{2}\right\}$ lie in the capacity region of the channel. The transmitter, then chooses a message $i \in\left\{1,2, \ldots, 2^{n R_{1}}\right\}$ and $k \in\left\{1,2, \ldots, 2^{n R_{2}}\right\}$ and transmits codewords $\mathbf{x}_{i}^{1}$ and $\mathbf{x}_{k}^{2}$ on the channel. Each receiver receives $\mathbf{y}^{1}$ and $\mathbf{y}^{2}$ given by

$$
\begin{aligned}
& \mathbf{y}^{1}=\mathbf{x}_{i}^{1}+\mathbf{x}_{k}^{2}+\tilde{\mathbf{z}}^{1}, \\
& \mathbf{y}^{2}=\mathbf{x}_{i}^{1}+\mathbf{x}_{k}^{2}+\tilde{\mathbf{z}}^{2},
\end{aligned}
$$

where $\tilde{\mathbf{z}}^{1}$ and $\tilde{\mathbf{z}}^{2}$ are Gaussian noise vectors with common standard deviation $\sigma$.

The channel coding problem for a two-user Gaussian multi-access channel refers to the problem of constructing a code $\mathcal{C}^{\mathrm{MCC}}\left[n, R_{1}, R_{2}, \sigma, P, \alpha, \epsilon\right]$, where $\alpha \in[0,1]$ is a fraction which determines the fraction of power allocated to receiver 1 . The outputs of $\mathcal{C}^{\mathrm{MCC}}\left[n, R_{1}, R_{2}, \sigma, P, \alpha, \epsilon\right]$ are:

(a) The codebooks $\mathcal{B}^{1}=\left\{\mathbf{x}_{i}^{1}\right\}_{i=1}^{2^{n R_{1}}}, \mathcal{B}^{2}=\left\{\mathbf{x}_{k}^{2}\right\}_{k=1}^{2^{n R_{2}}}$, satisfying

$$
\begin{aligned}
& \left\|\mathbf{x}_{i}^{1}\right\|^{2} \leq \alpha P \\
& \left\|\mathbf{x}_{k}^{2}\right\|^{2} \leq(1-\alpha) P .
\end{aligned}
$$

(b) The decoding functions $h^{1}: \mathbb{R}^{n} \rightarrow \mathcal{B}^{1}, h^{2}: \mathbb{R}^{n} \rightarrow \mathcal{B}^{2}$ that map each received codeword $\mathbf{y}^{1}, \mathbf{y}^{2}$ to one of the codewords in $\mathcal{B}^{1}$ and $\mathcal{B}^{2}$, respectively, so that the average probability of error satisfies

$$
\begin{gathered}
\frac{1}{2^{n R_{1}}} \sum_{i=1}^{2^{n R_{1}}} \mathbb{P}\left[h^{1}\left(\mathbf{y}^{1}\right) \neq i \mid m^{1}=i\right] \leq \epsilon, \\
\frac{1}{2^{n R_{2}}} \sum_{k=1}^{2^{n R_{2}}} \mathbb{P}\left[h^{2}\left(\mathbf{y}^{2}\right) \neq k \mid m^{2}=k\right] \leq \epsilon .
\end{gathered}
$$

As before, the capacity region $\mathcal{R}_{n}^{\mathrm{MCC}}[P, \sigma, \epsilon]$ is defined as the set of all rate pairs $\left(R_{1}, R_{2}\right)$ such that there exists a code $\mathcal{C}_{n}^{\mathrm{IC}}\left[P, \alpha, \sigma, R_{1}, R_{2}, \epsilon\right]$ for some $\alpha \in[0,1]$. 


\section{Capacity Characterization and Optimal Coding}

In this section, we begin by obtaining a relationship between the capacity regions of a multi-cast channel and an interference channel, respectively. In particular, we show that the capacity characterization of a multi-cast channel is a special case of an interference channel.

Proposition 44. Let $\mathcal{R}_{n}^{\mathrm{MCC}}[P, \sigma, \epsilon]$ be the finite capacity region of a multi-cast channel, and $\mathcal{R}_{n}^{\mathrm{IC}}\left[P_{1}, P_{2}, \sigma, \epsilon\right]$ be the finite capacity region of a two-user interference channel. Then

$\mathcal{R}_{n}^{M C C}[P, \sigma, \epsilon]=\left\{\left(R_{1}, R_{2}\right) \mid \exists \alpha \in[0,1]\right.$ such that $\left.\left(R_{1}, R_{2}\right) \in \mathcal{R}_{n}^{I C}[\alpha P,(1-\alpha) P, \sigma, \epsilon]\right\}$

Proof. Let $\left(R_{1}, R_{2}\right)$ be a rate pair such that for some $\alpha \in[0,1]$ and

$$
\left(R_{1}, R_{2}\right) \in \mathcal{R}_{n}^{\mathrm{IC}}[\alpha P,(1-\alpha) P, \sigma, \epsilon]
$$

Then there exists codebooks $\mathcal{B}^{1}, \mathcal{B}^{2}$ and decoding functions $h^{1}(\cdot), h^{2}(\cdot)$ such that

$$
\begin{array}{r}
\left\|\mathbf{x}_{i}^{1}\right\|^{2} \leq \alpha P, \forall \mathbf{x}_{i}^{1} \in \mathcal{B}^{1}, \\
\left\|\mathbf{x}_{k}^{2}\right\|^{2} \leq(1-\alpha) P, \forall \mathbf{x}_{k}^{2} \in \mathcal{B}^{1}, \\
\frac{1}{2^{n R_{1}}} \sum_{i=1}^{2^{n R_{1}}} \mathbb{P}\left[h^{1}\left(\mathbf{y}^{1}\right) \neq i \mid m^{1}=i\right] \leq \epsilon, \\
\frac{1}{2^{n R_{2}}} \sum_{k=1}^{2^{n R_{2}}} \mathbb{P}\left[h^{2}\left(\mathbf{y}^{2}\right) \neq k \mid m^{2}=i\right] \leq \epsilon .
\end{array}
$$

Therefore, the codebooks $\mathcal{B}^{1}, \mathcal{B}^{2}$ and decoding functions $h^{1}(\cdot), h^{2}(\cdot)$ satisfy the properties for a two user multi-access channel when the transmitter chooses $\alpha$ as the fraction to distribute power among the receivers.

Now suppose that $\left(R_{1}, R_{2}\right) \in \mathcal{R}_{n}^{\mathrm{MCC}}[P, \sigma, \epsilon]$, then $\exists \alpha \in[0,1], \mathcal{B}^{1}, \mathcal{B}^{2}$ and decoding 
functions $h^{1}(\cdot), h^{2}(\cdot)$ such that

$$
\begin{array}{r}
\left\|\mathbf{x}_{i}^{1}\right\|^{2} \leq \alpha P, \forall \mathbf{x}_{i}^{1} \in \mathcal{B}^{1}, \\
\left\|\mathbf{x}_{k}^{2}\right\|^{2} \leq(1-\alpha) P, \forall \mathbf{x}_{k}^{2} \in \mathcal{B}^{1}, \\
\frac{1}{2^{n R_{1}}} \sum_{i=1}^{2^{n R_{1}}} \mathbb{P}\left[h^{1}\left(\mathbf{y}^{1}\right) \neq i \mid m^{1}=i\right] \leq \epsilon, \\
\frac{1}{2^{n R_{2}}} \sum_{k=1}^{2^{n R_{2}}} \mathbb{P}\left[h^{2}\left(\mathbf{y}^{2}\right) \neq k \mid m^{2}=i\right] \leq \epsilon .
\end{array}
$$

Therefore, $\left(R_{1}, R_{2}\right) \in \mathcal{R}_{n}^{\mathrm{IC}}[\alpha P,(1-\alpha) P, \sigma, \epsilon]$. This concludes the proof.

Proposition 44 allows us to transform the problem of characterizing the capacity region of a multi-cast channel into the problem of characterizing the capacity region of an interference channel. In what follows, we present the algorithm (Algorithm 4) which we use to characterize the capacity region while simultaneously constructing the optimal code. The algorithm consists of two parts: (a) the Encoding Algorithm (Algorithm 10), and (b) the Decoding Algorithm (Algorithm 11).

Algorithm 10 is based on the feasibility problem with decision variables:

(a) The fraction $\alpha \in[0,1]$ that determines the distribution of power among the receivers;

(b) Variables $\left\{\mathbf{x}_{i}^{1}\right\}_{i=1}^{2^{n R_{1}}},\left\{\mathbf{x}_{k}^{2}\right\}_{k=1}^{2^{n R_{2}}}$ that represent the codewords for Users 1 and 2, respectively;

(c) Binary variables $v_{i}^{1}, v_{k}^{2}, v_{i k}^{1}, v_{i k}^{2}$ that are used to identify the subset of codewords which will be decoded correctly with probability at least $1-\epsilon^{\frac{1}{4}}$. In particular:

(1) $v_{i}^{1}=1$, if for codeword $i$ of User 1 , the average probability of error is at most $\epsilon^{\frac{1}{4}}$, that is,

$$
\frac{1}{2^{n R_{2}}} \sum_{k=1}^{2^{n R_{2}}} \mathbb{P}\left[g^{1}\left(\mathbf{y}^{1}\right) \neq i \mid m^{1}=i, m^{2}=k\right] \leq \epsilon^{\frac{1}{4}}
$$


and $v_{i}^{1}=0$, otherwise, where $g^{1}(\cdot)$ is defined in (4.66). The binary variables $v_{k}^{2}, \forall k=1, \ldots, 2^{n R_{2}}$ are defined in a similar fashion.

(2) $v_{i k}^{1}=1$, if whenever $m^{1}=i$ and $m^{2}=k$, User 1 is able to correctly decode with probability at least $1-\epsilon^{\frac{1}{4}}$, that is,

$$
\mathbb{P}\left[g^{1}\left(\mathbf{y}^{1}\right) \neq i \mid m^{1}=i, m^{2}=k\right] \leq \epsilon^{\frac{1}{4}}
$$

and $v_{i k}^{1}=0$ otherwise. The variables $v_{i k}^{2}, \forall i=1, \ldots, 2^{n R_{2}}, \forall k=1, \ldots, 2^{n R_{1}}$ are defined in a similar fashion.

(d) Binary variables $\left\{v_{i k i^{\prime} k^{\prime} t}^{1}, v_{i k i^{\prime} k^{\prime} t}^{2}\right\}_{t=1}^{T}$ are auxiliary variables which allow us to constrain the probability of incorrect decoding for codewords identified by $v_{i}^{1}, v_{k}^{2}, v_{i k}^{1}, v_{i k}^{2}$. In particular, for codewords $i, i^{\prime}, k, k^{\prime}$ with $v_{i k}^{1}=1$ and $v_{i^{\prime} k^{\prime}}^{1}=1$,

$$
v_{i k i^{\prime} k^{\prime} t}^{1}= \begin{cases}1, & \text { if }\left\|\left(\mathbf{x}_{i}^{1}+h_{12} \mathbf{x}_{k}^{2}\right)-\left(\mathbf{x}_{i^{\prime}}^{1}+h_{12} \mathbf{x}_{k^{\prime}}^{2}\right)+\mathbf{z}_{t}\right\| \geq\left\|\mathbf{z}_{t}\right\| \\ 0, & \text { otherwise. }\end{cases}
$$

We next present the algorithm that allows us to correctly characterize the capacity region. 

\begin{tabular}{l}
\hline Algorithm 10 Encoding Algorithm for two-user Multi-cast Channel \\
Input : $n, R_{1}, R_{2}, \sigma, P, \epsilon, \nu$. \\
Output : Fraction $\alpha$, codewords $\mathbf{x}_{i}^{1}, \mathbf{x}_{k}^{2}$, and binary variables $v_{i}^{1}, v_{k}^{2}, v_{i k}^{1}, v_{i k}^{2}$, \\
$\left\{v_{i k i^{\prime} k^{\prime} t}^{1}, v_{i k i^{\prime} k^{\prime} t}^{2}\right\}_{t=1}^{T}$.
\end{tabular}

Algorithm :

1. Calculate the quantities $T, M_{0}$, and the set of equidistant vectors $\mathcal{Z}=\left\{\mathbf{z}_{t}\right\}_{t=1}^{T}$.

2. Check the feasibility of the constraints

$$
\begin{aligned}
& \left\|\mathbf{x}_{i}^{1}\right\|^{2} \leq n \alpha P \quad \forall i \\
& \left\|\mathrm{x}_{k}^{2}\right\|^{2} \leq n(1-\alpha) P \quad \forall k, \\
& \left\|\mathbf{x}_{i}^{1}-\mathbf{x}_{i^{\prime}}^{1}+h_{12}\left(\mathbf{x}_{k}^{2}-\mathbf{x}_{k^{\prime}}^{2}\right)+\mathbf{z}_{t}\right\| \\
& +\left(1-v_{i k i^{\prime} k^{\prime} t}^{1}\right) M_{0} \geq\left\|\mathbf{z}_{t}\right\|, \quad \forall i, i^{\prime}, k, k^{\prime}, t, \\
& v_{i k i^{\prime} k^{\prime} t}^{1} \leq v_{i k}^{1} \quad \forall i, i^{\prime}, k, k^{\prime}, t, \\
& v_{i k i^{\prime} k^{\prime} t}^{\mathbf{1}} \leq v_{i^{\prime} k^{\prime}}^{1} \quad \forall i, i^{\prime}, k, k^{\prime}, t \\
& \sum_{t=1}^{T} v_{i k i^{\prime} k^{\prime} t}^{1} \geq\left(1-\epsilon^{\frac{1}{4}}\right) T \cdot v_{i k}^{1} v_{i^{\prime} k^{\prime}}^{1} \quad \forall i, i^{\prime}, k, k^{\prime}, t \\
& v_{i k}^{1} \leq v_{i}^{1} \quad \forall i, k, \\
& \sum_{k=1}^{2^{n R_{2}}} v_{i k}^{1} \geq\left(1-\epsilon^{\frac{1}{4}}\right) 2^{n R_{2}} \cdot v_{i}^{1} \quad \forall i, \\
& \sum_{i=1}^{2^{n R_{1}}} v_{i}^{1} \geq\left(1-\epsilon^{\frac{1}{4}}\right) \cdot\left(1-2 \epsilon^{\frac{1}{4}}\right) \cdot 2^{n R_{1}} \\
& \left\|\mathbf{x}_{k}^{2}-\mathbf{x}_{k^{\prime}}^{2}+h_{21}\left(\mathbf{x}_{i}^{1}-\mathbf{x}_{i^{\prime}}^{1}\right)+\mathbf{z}_{t}\right\| \\
& +\left(1-v_{i k i^{\prime} k^{\prime} t}^{2}\right) M_{0} \geq\left\|\mathrm{z}_{t}\right\|, \quad \forall i, i, k, k^{\prime}, t, \\
& v_{i k i^{\prime} k^{\prime} t}^{2} \leq v_{i k}^{2} \quad \forall i, i^{\prime}, k, k^{\prime}, t, \\
& v_{i k i^{\prime} k^{\prime} t}^{2} \leq v_{i^{\prime} k^{\prime}}^{2} \quad \forall i, i^{\prime}, k, k^{\prime}, t \\
& \sum_{t=1}^{T} v_{i k i^{\prime} k^{\prime} t}^{2} \geq\left(1-\epsilon^{\frac{1}{4}}\right) T \cdot v_{i k}^{2} v_{i^{\prime} k^{\prime}}^{2}, \quad \forall i, i^{\prime}, k, k^{\prime} \\
& v_{i k}^{2} \leq v_{i}^{2} \quad \forall i, k, \\
& \sum_{k=1}^{2^{n R_{1}}} v_{i k}^{2} \geq\left(1-\epsilon^{\frac{1}{4}}\right) 2^{n R_{1}} v_{i}^{2} \quad \forall i, \\
& \sum_{i=1}^{2^{n R_{2}}} v_{i}^{2} \geq\left(1-\epsilon^{\frac{1}{4}}\right) \cdot\left(1-2 \epsilon^{\frac{1}{4}}\right) \cdot 2^{n R_{2}} \\
& v_{i}^{1}, v_{k}^{2}, v_{i k}^{1}, v_{i k}^{2}, v_{i k i^{\prime} k^{\prime} t}^{1}, v_{i k i^{\prime} k^{\prime} t}^{2} \in\{0,1\}, \quad \forall i, k, i^{\prime}, k^{\prime}, t .
\end{aligned}
$$

3. If feasible, then $\left(R_{1}, R_{2}\right)$ is achievable and the resulting codewords can be used to transmit messages. 
Algorithm 11 Decoding Algorithm for two-user Multi-cast Channel

Input : Received codewords $\mathbf{y}^{1}, \mathbf{y}^{2}$.

Output : Messages $i_{1}^{*}, i_{2}^{*}$.

Algorithm :

1. Solve

$$
\begin{aligned}
& i_{1}^{*}=g^{1}\left(\mathbf{y}^{1}\right)=\arg \min _{i \in\left\{1, \ldots, M_{1}\right\}: v_{i}^{1}=1}\left[\min _{k \in\left\{1, \ldots, M_{2}\right\}: v_{i k}^{1}=1}\left\|\mathbf{y}^{1}-\left(\mathbf{x}_{i}^{1}+h_{12} \mathbf{x}_{k}^{2}\right)\right\|\right], \\
& i_{2}^{*}=g^{2}\left(\mathbf{y}^{2}\right)=\arg \min _{k \in\left\{1, \ldots, M_{2}\right\}: v_{k}^{2}=1}\left[\min _{i \in\left\{1, \ldots, M_{1}\right\}: v_{i k}^{2}=1}\left\|\mathbf{y}^{1}-\left(\mathbf{x}_{i}^{2}+h_{21} \mathbf{x}_{k}^{1}\right)\right\|\right] .
\end{aligned}
$$

Algorithm 12 Capacity Characterization and Optimal Coding for the Two-User

Multi-cast Channel

Input : $R_{1}, R_{2}, P, \sigma, n, \epsilon, \nu$.

Output : Rank $r^{*}$, fraction $\alpha \in[0,1]$, codewords $\left\{\mathbf{x}_{i}^{1}\right\}_{i=1}^{2^{n R_{1}}},\left\{\mathbf{x}_{k}^{2}\right\}_{k=1}^{2^{n R_{2}}}$, and auxiliary binary variables $v_{i}^{1}, v_{k}^{2}, v_{i k}^{1}, v_{i k}^{2},\left\{v_{i k i^{\prime} k^{\prime} t}^{1}, v_{i k i^{\prime} k^{\prime} t}^{2}\right\}_{t=1}^{T}$.

\section{Algorithm :}

1. Solve the rank minimization semidefinite optimization problem that is equivalent to the feasibility check problem of constraints (4.123)-(4.140) to compute $r^{*}$.

2. If $r^{*}=1$, then $\left(R_{1}, R_{2}\right)$ is achievable using the fraction $\alpha$, codebooks $\mathcal{B}^{1}=$ $\left\{\mathbf{x}_{i}^{1}\right\}_{i=1}^{2^{n R_{1}}}, \mathcal{B}^{2}=\left\{\mathbf{x}_{k}^{2}\right\}_{k=1}^{2^{n R_{2}}}$ and the decoding functions (4.66), achieving an average decoding error probability of $\mathcal{O}\left(\epsilon^{\frac{1}{4}}\right)$. That is,

$$
\text { If } r^{*}=1 \text {, then }\left(R_{1}, R_{2}\right) \in \mathcal{R}_{n}^{\mathrm{MCC}}\left[P_{1}, P_{2}, \sigma, \epsilon^{\frac{1}{4}}\right] \text {. }
$$

3. If $r^{*} \geq 2$, then we can conclude using Theorem 45 that, $\left(R_{1}, R_{2}\right)$ cannot be achieved on a multi-cast channel with noise standard deviation $(1+2 \nu) \sigma$ with probability of error less than or equal to $\epsilon$. That is,

$$
\text { If } r^{*} \geq 2, \text { then }\left(R_{1}, R_{2}\right) \notin \mathcal{R}_{n}^{\mathrm{IC}}\left[P_{1}, P_{2},(1+2 \nu) \sigma, \epsilon\right] \text {. }
$$


We next present Theorem 45 that shows the correctness of Algorithm 12 .

Theorem 45. Let $r^{*}, \alpha^{*},\left\{\mathbf{x}_{i}^{1}\right\}_{i=1}^{2^{n R_{1}}},\left\{\mathbf{x}_{k}^{2}\right\}_{k=1}^{2^{n R_{2}}}$ be an optimal solution of the rankconstrained SDP formulation of (4.123-4.140).

(a) If $r^{*}=1$, then $\left(R_{1}, R_{2}\right)$ is achievable using the fraction $\alpha^{*}$, codebooks $\mathcal{B}^{1}=$ $\left\{\mathbf{x}_{i}^{1}\right\}_{i=1}^{2^{n R_{1}}}, \mathcal{B}^{2}=\left\{\mathbf{x}_{k}^{2}\right\}_{k=1}^{2^{n R_{2}}}$ and the decoding functions (4.141), achieving a decoding error probability of $\epsilon$. That is,

$$
\text { If } r^{*}=1, \text { then }\left(R_{1}, R_{2}\right) \in \mathcal{R}_{n}^{M C C}\left[P_{1}, P_{2}, \sigma, \epsilon\right] \text {. }
$$

(b) If $r^{*} \geq 2$, then $\left(R_{1}, R_{2}\right)$ cannot be achieved on a Gaussian multi-cast channel with noise standard deviation $(1+2 \nu) \sigma$ with probability of error less than or equal to є. That is,

$$
\text { If } r^{*} \geq 2, \text { then }\left(R_{1}, R_{2}\right) \notin \mathcal{R}_{n}^{M C C}\left[P_{1}, P_{2},(1+2 \nu) \sigma, \epsilon\right]
$$

Proof. The proof follows from the reduction of a multi-cast channel to an interference channel based on Proposition 44.

\subsection{Implementation and Computational Results}

\subsubsection{Computational Experiments : Gaussian Interference Channel}

In each of the Gaussian channels, we are required to solve a rank-constrained semidefinite optimization problem. We use the iterative algorithm (Algorithm 3), which was developed by Fazell et al. [2003], to solve these problems. 
Algorithm 13 Solving Rank Constrained SDPs

Input: $A, \delta$.

Output: A rank one PSD matrix $X^{K}$.

- Step 1 : Solve the convex optimization problem

$$
\begin{gathered}
\min \operatorname{Tr}(X) \\
\text { s.t. } \tilde{A} \bullet X \leq 0, \\
X \succeq 0,
\end{gathered}
$$

and let $X^{0}$ denote the optimal solution.

- Step 2 : For each iteration $k=1, \ldots, K$, solve the optimization problem

$$
\begin{aligned}
& \min \operatorname{Tr}\left(\left(X^{k-1}+\delta I\right)^{-1} X\right) \\
& \text { s.t. } \tilde{A} \bullet X \leq 0, \\
& X \succeq 0 .
\end{aligned}
$$

Using this algorithm, we perform the experiments to characterize the rate regions of interference channels. To do this, we choose a rate pair and check whether it is feasible to the optimization problem (4.67). In particular, we do the following

1. Choose a rate pair $\left\{R_{1}, R_{2}\right\}$ using inner and outer bounds from the literature (see Verdu and McLaughlin [1998]).

2. Input $M_{1}=2^{n R_{1}}, M_{2}=2^{n R_{2}}$ and $\mathbb{U}^{n}$ to the optimization problem and check whether $n$ is feasible, for $n=20,25,30,35,40$. 


\section{Achieving Carleial's rate pairs}

We consider the rate region proposed by Carleial [1975]. He showed that the rate pairs

$$
\left\{\left(D_{1}, D_{2}\right),\left(C_{1}, \min \left(D_{2}, T_{2}\right)\right),\left(\min \left(D_{1}, T_{1}\right), C_{2}\right),\left(\min \left(C_{1}, T_{1}\right), \min \left(C_{2}, T_{2}\right)\right)\right\}
$$

where

$$
\begin{aligned}
C_{1}=\frac{1}{2} \log \left(1+P_{1}\right), \quad C_{2}=\frac{1}{2} \log \left(1+P_{2}\right), & D_{1}=\frac{1}{2} \log \left(\frac{1+P_{1}}{1+h_{12} P_{2}}\right), \\
D_{2}=\frac{1}{2} \log \left(\frac{1+P_{2}}{1+h_{21} P_{1}}\right), \quad T_{1}=\frac{1}{2} \log \left(\frac{1+h_{21} P_{1}}{1+P_{2}}\right), & T_{2}=\frac{1}{2} \log \left(\frac{1+h_{12} P_{2}}{1+P_{1}}\right) .
\end{aligned}
$$

We verify that these rates are indeed achievable and also show, in some cases, that we can do better. For example, when we use the parameters $P_{1}=1.5, P_{2}=2, h_{12}=$ $0.3, h_{21}=0.3$, we obtain $C_{1}=0.66096, C_{2}=0.7924, D_{1}=0.3219$, and $D_{2}=$ 0.5244 . the rate region obtained by the optimization approach is compared against this Carleial's rate region in Figure 4-1.

\section{Achieving the rate regions of Han and Kobayashi Han and Kobayashi [1981]}

We next consider the rate regions of Han and Kobayashi [1981]. The Han-Kobayashi rate region is given by

$$
\mathcal{R}=\left\{\begin{array}{c}
R_{1} \leq \frac{1}{2} \log \left(1+P_{1}\right) \\
R_{2} \leq \frac{1}{2} \log \left(1+P_{2}\right) \\
R_{1}+R_{2} \leq \min \left[\frac{1}{2} \log \left(1+P_{1}+h_{12} P_{2}\right), \frac{1}{2} \log \left(1+P_{2}+h_{21} P_{1}\right)\right]
\end{array}\right\} .
$$

We present results for a choice of parameters $P_{1}=P_{2}=2, h_{12}=h_{21}=1.5$ in Figure 4-2. 


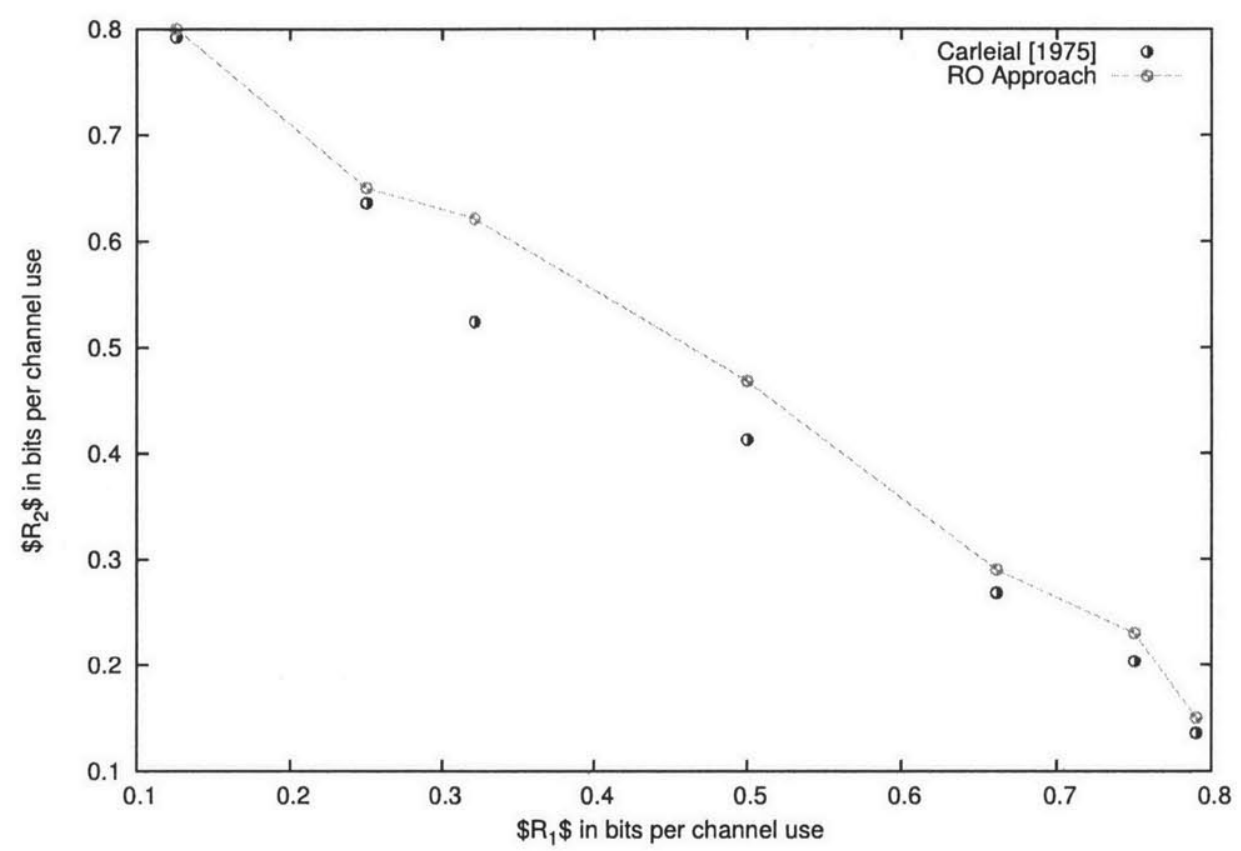

Figure 4-1: Comparison with Carleial's Rate Region

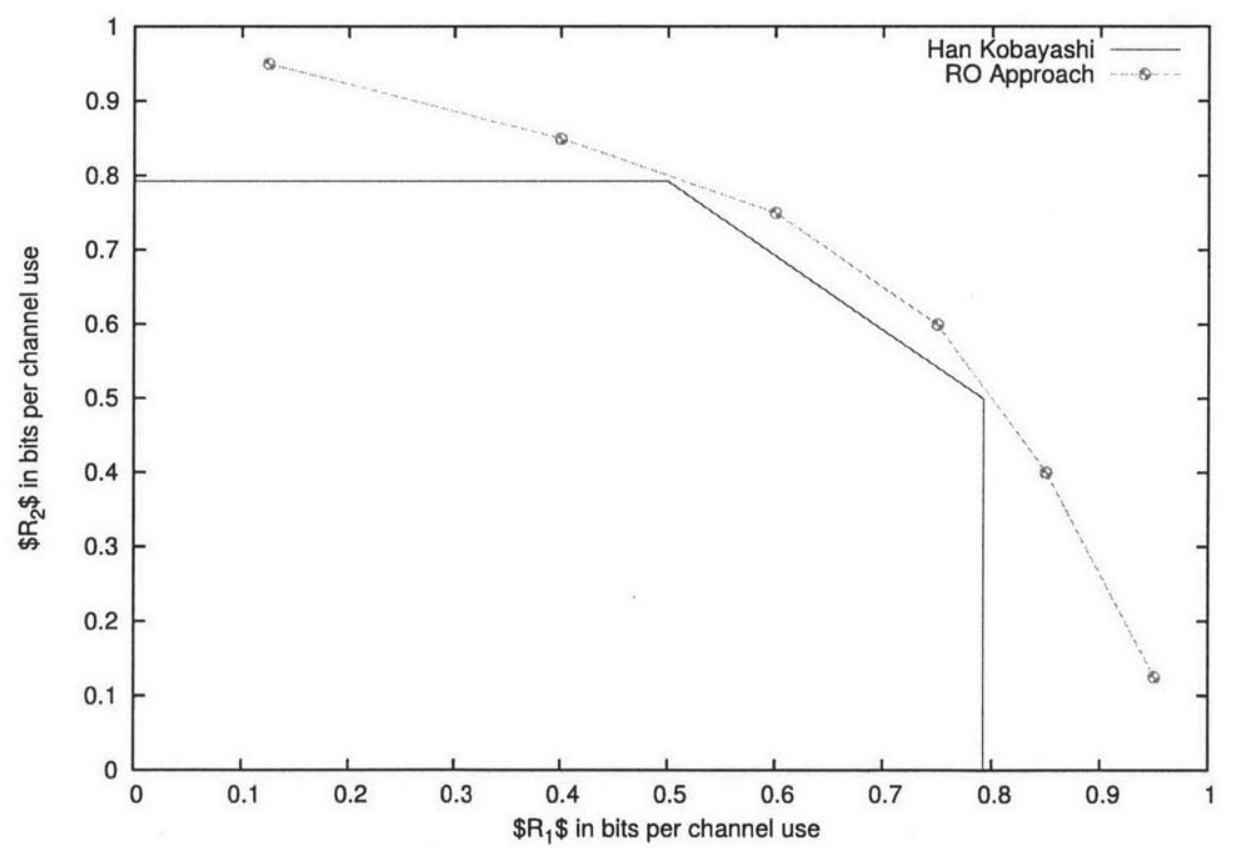

Figure 4-2: Comparison with Han-Kobayashi Rate Region 


\section{Comparison with existing bounds}

In the next experiment, we compare the rate region obtained in our framework with that obtained by others. In particular, we compare with the Han-Kobayashi region, Tse's region and the Broadcast channel bound. The results are presented in Figure 4-3.

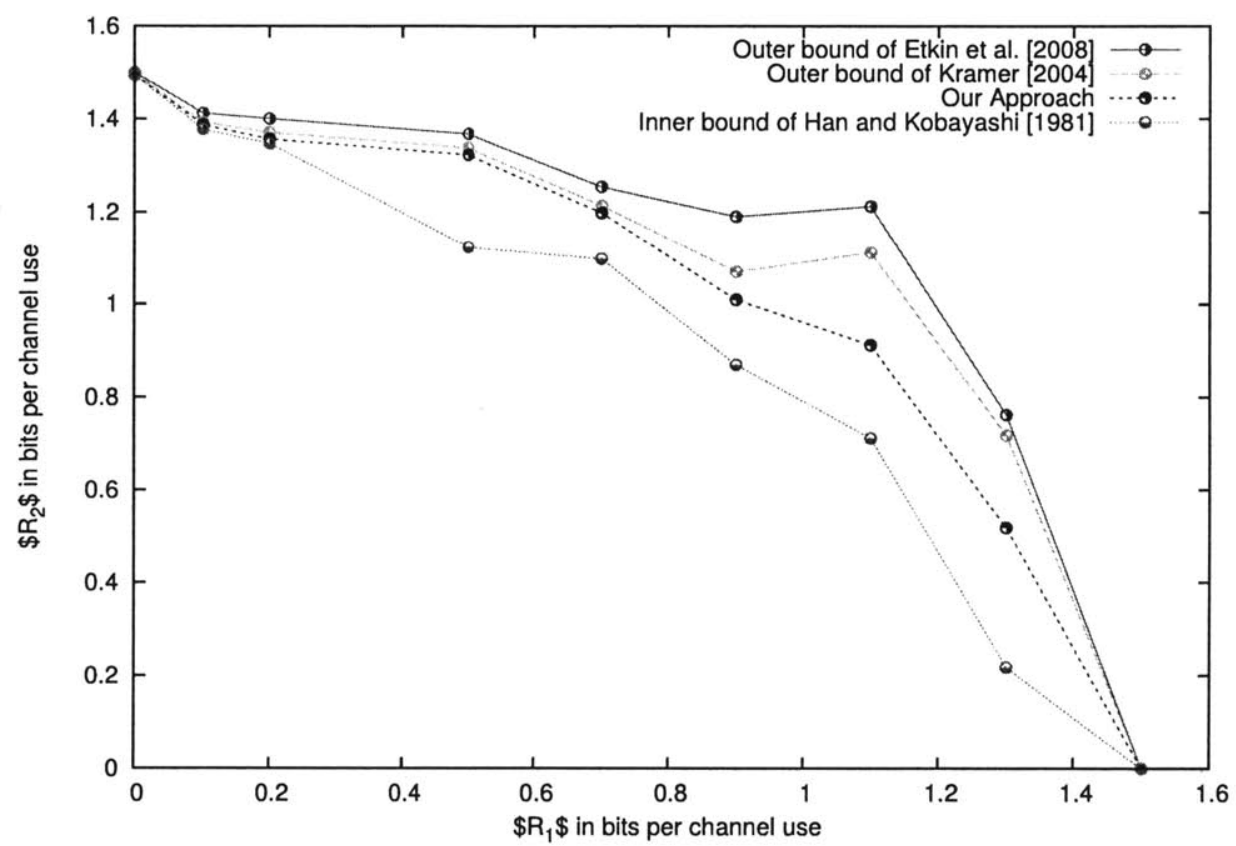

Figure 4-3: Two-user Gaussian interference channel - $P_{1}=10, P_{2}=20, h_{12}=$ $0.2, h_{21}=0.25$

\subsubsection{Computational Experiments : Exponential Channel}

In case of the exponential channels, the channel coding optimization problem is a mixed-integer linear optimization problem. We use the commercially available solver CPLEX to solve these problems. We note that the computational times for verifying whether a rate pair is achievable for the exponential channel, are very encouraging and we were able to solve instances of the order 200,000 codewords using CPLEX. 


\subsection{Conclusion}

In this chapter, we proposed an uncertainty set based approach to model the noise in a communication channel. This approach was motivated by the concept of "typical sequences". When such an approach is used, the channel coding problems can be reduced to instances of Robust Optimization problems. We also showed how these optimization problems can be used to characterize the capacity regions of Interference Channel, and Multi-cast Channel. Towards the end, we showed the practicality of this approach by showing that moderately sized instances can be solved to optimality. This approach clearly opens up new directions and it remains to be seen how this approach can be used to compute the capacity region of Relay Channels and Channels with Memory. 


\section{Chapter 5}

\section{Conclusion}

In this thesis, we revisited some of the major successes in the $20^{\text {th }}$ century for stochastic analysis. In all these examples, together with considerable success, came challenges when researchers and practitioners desired to generalize the problems studied to mutliple dimensions. It is our contention that stochastic analysis, based on the primitives of the Kolmogorov axioms and the concept of random variables was not intended to provide a tool for efficient computation. Rather it was intended to put the theory on a firm ground and give insights on the modeling of stochastic phenomena. In retrospect, given the historical developments and intentions of the originators, the computational challenges that stochastic analysis has faced, when attempting to solve problems in multiple dimensions, should have been anticipated.

Possibly because the development of modern optimization happened at about the same time as the development of the digital computer, optimization had from its very beginning efficient computation as its intention. Correspondingly, optimization has succeeded remarkably to solve problems in multiple dimensions. Given its success, it seems natural to us to apply optimization to solve problems of stochastic analysis in multiple dimensions.

In all three examples we addressed we have implemented the proposed approach and have included in the thesis tables and figures with computational evidence in concrete examples in order to show that the approach of stochastic analysis based on optimization is capable of solving problems numerically in ways that, in our opinion, 
go beyond the current state of the art of stochastic analysis. The types of optimization problems that were required to be solved ranged from linear and semidefinite to discrete, bilinear and rank optimization problems. We anticipate that this research program, in addition to advancing stochastic analysis, will also advance optimization as it will reveal new optimization problems that need to be addressed. 


\section{Bibliography}

L. M. Ausubel. An efficient ascending-bid auction for multiple objects. American Economic Review, 94(5):1452-1475, 2004.

C. Bandi and D. Bertsimas. Optimal design for multi-item auctions: A robust optimization approach. Submitted for publication to Mathematics of Operations Research, 2011.

C. Bandi and D. Bertsimas. Tractable stochastic analysis via robust optimization. Mathematical Programming, Series B, 134, 2012a.

C. Bandi and D. Bertsimas. Network information theory via robust optimization. Submitted for Publication to IEEE Transactions on Information Theory, 2012b.

C. Bandi, D. Bertsimas, and N. Youssef. Robust queueing theory. Submitted for publication to Operations Research, 2011.

C. Bandi, D. Bertsimas, and N. Youssef. Robust transient analysis of queueing systems. Working Paper, 2012.

A.-L. Barabasi. The origin of bursts and heavy tails in human dynamics. NATURE, 435:207, 2005. URL doi:10.1038/nature03459.

A. Ben-Tal and A. Nemirovski. Robust convex optimization. Mathematics of Operations Research, 23(4):769-805, 1998.

A. Ben-Tal and A. Nemirovski. Robust solutions to uncertain programs. Operations Research Letters, 25:1-13, 1999.

A. Ben-Tal and A. Nemirovski. Robust solutions of linear programming problems contaminated with uncertain data. Mathematical Programming, 88:411-424, 2000.

A. Ben-Tal, L. E. Ghaoui, and A. Nemirovski. Robust Optimization. Princeton University Press, 2009.

J. Benoit and V. Krishna. Multiple-object auctions with budget constrained bidders. Review of Economic Studies, 68:155-179, 2001.

T. Benson, A. Akella, and D. A. Maltz. Network traffic characteristics of data centers in the wild. In Proceedings of the 10th annual conference 
on Internet measurement, IMC '10, pages 267-280, New York, NY, USA, 2010. ACM. ISBN 978-1-4503-0483-2. doi: 10.1145/1879141.1879175. URL http://doi .acm.org/10.1145/1879141.1879175.

E. Beran, L. Vandenberghe, and S. Boyd. A global bmi algorithm based on the generalized benders decomposition. Proceedings of the European Control Conference, pages 1074-1082, 1997.

D. Bertsimas. An analytic approach to a general class of $G / G / s$ queueing systems. Operations Research, 38:139-155, 1990.

D. Bertsimas and M. Sim. Robust discrete optimization and network flows. Mathematical Programming, 98:49-71, 2003.

D. Bertsimas and M. Sim. The price of robustness. Operations Research, 52(1):35-53, 2004.

D. Bertsimas, D. Brown, and C. Caramanis. Theory and applications of robust optimization. SIAM Review, 53(3):464-501, 2011.

F. Black and M. Scholes. Pricing of options and corporate liabilities. Jourmal of Political Economy, 81:637-654, 1973.

C. Borgs, J. T. Chayes, N. Immorlica, M. Mahdian, and A. Saberi. Multi-unit auctions with budget constrained bidders. ACM Conference on Electronic Commerce, pages 44-51, 2005.

A. Borodin, J. Kleinberg, P. Raghavan, M. Sudan, and D. Williamson. Adversarial queueing theory. Journal of $A C M, 2001$.

J. L. Boudec and P. Thiran. Network Calculus: A Theory of Deterministic Queuing Systems for the Internet. LNCS, Springer, 2001.

S. Brusco and G. Lopomo. Simultaneous ascending bid auctions with privately known budget constraints. Journal of Industrial Economics, 56(1):113-142, 2008.

J. Bulow and P. Klemperer. Auctions versus negotiations. American Economic Review, 86:180-194, 1996.

A. Burchard, F. Ciucu, and J. Liebeherr. On superlinear scaling of network delays. IEEE/ACM Transactions on Networking, 19(4):1043-1056, 2011.

A. Burchard, F. Ciucu, and J. Liebeherr. Delay bounds in communication networks with heavy-tailed and self-similar traffic. IEEE Transactions on Information Theory, 58(2):1010-1024, 2012.

P. Burke. The output of a queueing system. Operations Research, 4(6):699-704, 1956.

A. B. Carleial. A case where interference does not reduce capacity. IEEE Transactions on Information Theory, 21(5):569-570, 1975. 
S. Chawla, J. D. Hartline, D. L. Malec, and B. Sivan. Multi-parameter mechanism design and sequential posted pricing. STOC, pages 311-320, 2010.

Y. Che and J. Gale. Expected revenue of the all-pay auctions and first-price sealed bid auctions with budget constraints. Economic Letters, 50:373-380, 1996.

Y. Che and J. Gale. The optimal mechanism for selling to a budget constrained buyer. Journal of Economic Theory, 92(2):198-233, 2000.

F. Ciucu. Network calculus delay bounds in queueing networks with exact solutions. In L. Mason, T. Drwiega, and J. Yan, editors, Managing Traffic Performance in Converged Networks, volume 4516 of Lecture Notes in Computer Science, pages 495-506. Springer Berlin / Heidelberg, 2007.

F. Ciucu and $\mathrm{O}$. Hohlfeld. On computing bounds on average backlogs and delays with network calculus. In 2010 IEEE International Conference on Communications, pages 1-5, 2010.

F. Ciucu, A. Burchard, and J. Liebeherr. A network service curve approach for the stochastic analysis of networks. ACM Sigmetrics, 2005.

E. H. Clarke. Multipart pricing of public goods. Public Choice, 11:17-33, 1971.

S. Cook. The complexity of theorem-proving procedures. Conference Record of Third Annual ACM Symposium on Theory of Computing, 1:151-158, 1971.

T. M. Cover and J. A. Thomas. Elements of Information Theory. Wiley, New York, 2006.

J. Cremer and R. P. McLean. Full extraction of the surplus in bayesian and dominant strategy auctions. Econometrica, 56(6):1247-57, 1988.

M. Crovella. The relationship between heavy-tailed file sizes and self-similar network traffic. INFORMS Applied Probability Conference, 1997.

M. Crovella. Performance evaluation with heavy tailed distributions. Computer Performance Evaluation, LNCS, Springer, 1786:1-9, 2000.

R. L. Cruz. A calculus for network delay, part i: Network elements in isolation. IEEE Transactions on Information Theory, 37:114-131, $1991 \mathrm{a}$.

R. L. Cruz. A calculus for network delay, part ii: Network analysis. IEEE Transactions on Information Theory, 37:132-141, 1991b.

C.S.Chang. Performance Guarantees in Communication Networks. Springer, 2001.

G. B. Dantzig. Programming of interdependent activities: II mathematical model. Econometrica, 17:200-211, 1949. 
G. B. Dantzig. Linear programming and extensions. Princeton University Press and the RAND Corporation, 1963.

P. Dhangwatnotai, T. Roughgarden, and Q. Yan. Revenue maximization with a single sample. Proceedings of 12th ACM Conference on Electronic Commerce, 2010.

S. Dobzinski, R. Lavi, and N. Nisan. Multi-unit auctions with budget limits. FOCS, pages $260-269,2008$.

L. El-Ghaoui and H. Lebret. Robust solutions to least-square problems to uncertain data matrices. SIAM Journal on Matrix Analysis and Applications, 18:1035-1064, 1997.

L. El-Ghaoui, F. Oustry, and H. Lebret. Robust solutions to uncertain semidefinite programs. SIAM Journal on Optimization, 9:33-52, 1998.

M. El-Taha and S. Stidham. Sample-Path Analysis of Queueing Systems. Springer, 1999.

A. K. Erlang. The theory of probabilities and telephone conversations. Nyt Tidsskrift for Matematik, B, 20, 1909.

R. Etkin, D. N. C. Tse, and H. Wang. Gaussian interference channel capacity to within one bit. IEEE Transactions on Information Theory, 54(12):5534-5562, 2008.

M. Fazell, H. Hindi, and S. P. Boyd. Log-det heuristic for matrix rank minimization with applications to hankel and euclidean distance matrices. Proceedings American Control Conference, 3:2156-2162, 2003.

A. Feinstein. A new basic theorem of information theory. IRE Transactions on Information Theory, 4:2-22, 1954.

P. L. Gall. The stationary $G / G / s$ queue. Journal of Applied Mathematics and Stochastic Analysis, 11:59-71, 1998.

G. Gallager and A. Parekh. A generalized processor sharing approach to flow control in integrated services networks: the multiple node case. IEEE/ACM Transactions on Networking, 2:137-150, 1994.

A. E. Gamal and T. M. Cover. Multiple user information theory. Proceedings of the IEEE, 68:1466-1483, 1980.

D. Gamarnik. Using fluid models to prove stability of adversarial queueing networks. IEEE Transactions on Automatic Control, 4:741-747, 2000.

D. Gamarnik. Stability of adaptive and non-adaptive packet routing policies in adversarial queueing networks. SIAM Journal on Computing, pages 371-385, 2003.

B. Gnedenko and A. Kolmogorov. Limit Distributions for Sums of Independent Random Variables. Addison Wesley, Reading, Ma, 1968. 
A. Goel. Stability of networks and protocols in the adversarial queueing model for packet routing. Proc. 10th ACM-SIAM Symposium on Discrete Algorithms, 1999.

A. Goldberg, J. Hartline, A. Karlin, M. Saks, and A. Wright. Competitive auctions. Games and Economic Behavior, 55(2):242-269, 2006.

T. Groves. Incentives in teams. Econometrica, 45:617-631, 1977.

T. S. Han and K. Kobayashi. A new achievable rate region for the interference channel. IEEE Transactions on Information Theory, 27(1):49-60, 1981.

J. Jackson. Networks of waiting lines. Operations Research, 5:518-521, 1957.

P. Jelenkovic, A. Lazar, and N. Semret. The effect of multiple time scales and subexponentiality in mpeg video streams on queueing behavior. IEEE Journal of Selected Areas in Communication, 15(6):1052-1071, 1997.

Y. Jiang. In 2012 International Conference on Computing, Networking and Commincations, pages 638-644, 2012.

Y. Jiang and Y. Liu. Stochastic Network Calculus. Springer, 2008.

R. M. Karp. Complexity of Computer Computations, chapter Reducibility among combinatorial problems, pages 85-103. Plenum Press, New York, NY, 1972.

J. Kiefer and J. Wolfowitz. On the theory of queues with many servers. Transactions of the American Mathematical Society, 78(1):1-18, 1955.

J. Kingman. Inequalities in the theory of queues. Journal of the Royal Statistical Society, 32:102-110, 1970.

J. Kingman. 100 years of queueing. Proceedings of Conference on The Erlang Centennial, pages 3-13, 2009.

P. Klemperer. Auction theory: A guide to the literature. Journal of Economic Surveys, 13(3):227-286, 1999.

V. Krishna. Auction Theory. San Diego : Academic Press, 2002.

P. Kuehn. Approximate analysis of general queueing networks by decomposition. IEEE Trans. Comm., 1979.

R. Kumar, P. Raghavan, S. Rajagopalan, D. Sivakumar, A. Tomkins, and E. Upfal. Stochastic models for the web graph. Proceedings of the 41 st Annual Symposium on Foundations of Computer Science, pages 57-65, 2000.

J.-J. Laffont and J. Robert. Optimal auction with financially constrained buyers. Economic Letters, 52(2):181-186, 1996.

W. Leland, M. Taqqu, and D. Wilson. On the self-similar nature of Ethernet traffic. ACM SIGCOMM Computer Communication Review, 25(1):202-213, 1995. 
D. V. Lindley. The theory of queues with a single server. Mathematical Proceedings of the Cambridge Philosophical Society, 1952.

C. Loboz. Cloud resource usage - heavy tailed distributions invalidating traditional capacity planning models. J. Grid Comput., 10(1):85-108, 2012.

A. Malakhov and R. Vohra. Single and multi-dimensional optimal auctions - a network approach. CMS-EMS DP No.1397, Northwestern University,, 2004.

A. M. Manellia and D. R. Vincent. Multidimensional mechanism design: Revenue maximization and the multiple-good monopoly. Journal of Economic Theory, $137(1): 153-185,2007$.

E. Maskin. Auctions, development, and privatization: Efficient auctions with liquidity constrained buyers. European Economic Review, 44:667-681, 2000.

R. B. Myerson. Optimal auction design. Mathematics of Operations Research, 6(1): 58-73, 1981.

N. Nisan, T. Roughgarden, E. Tardos, and V. V. Vazirani. Algorithmic Game Theory. Cambridge University Press, New York, NY, USA, 2007.

J. Nolan. Numerical calculation of stable densities and distribution functions. Stochastic Models, 1997.

M. Pai and R. Vohra. Optimal auctions with financially constrained bidders. Working paper, 2008.

C. H. Papadimitriou and G. Pierrakos. On optimal single-item auctions. STOC, 2011.

F. Pollaczek. Problèmes stochastiques posés par le phénomène de formation d'une queue d'attente à un guichet et par des phénomènes apparentés. Mémorial des Sciences Mathématiques, Paris, 1957.

A. Ronen. On approximating optimal auctions. ACM Conference on Electronic Commerce, pages 11-17, 2001.

I. Sason. On achievable rate regions for the gaussian interference channel. IEEE Transactions on Information Theory, 50(6):1345-1356, 2004.

C. E. Shannon. A mathematical theory of communication. Bell System Technical Journal, 27:379-423, 1948a.

C. E. Shannon. A mathematical theory of communication. Bell System Technical Journal, 27:379-423, 1948b.

H. D. Sherali and A. Alameddine. A new reformulation linearization technique for bilinear programming problems. Journal of Global Optimization, 2:379-410, 1992. 
J. Thanassoulis. Haggling over substitutes. Journal of Economic Theory, 117(2): 217-245, 2004.

S. Verdu. Fifty years of shannon theory. IEEE Transactions on Information Theory, 44:2057-2078, 1998.

S. Verdu and S. W. McLaughlin. Information Theory: 50 Years of Discovery. IEEE Press, New York, 1998.

W. Vickrey. Counterspeculation, auctions, and competitive sealed tenders. The Journal of Finance, 16(1):8-37, 1961.

R. Vohra. Mechanism Design: A Linear Programming Approach. Cambridge University Press, New York, NY, USA, 2011.

W. Whitt. The queueuing network analyzer. Bell System Technical Journal, pages 2779-2813, 1983.

W. Whitt. The impact of a heavy-tailed service-time distribution upon the $\mathrm{m} / \mathrm{gi} / \mathrm{s}$ waiting-time distribution. Queueing Systems, 36:71-87, 2000.

W. Willinger, V. Paxson, and M. S. Taqqu. Self-similarity and heavy tails: Structural modeling of network traffic. A Practical Guide to Heavy Tails: Statistical Techniques and Applications, 1998.

R. B. Wilson. Nonlinear Pricing. Oxford University Press, 1997.

A. Wyner. Random packing and coverings of the unit n-sphere. Bell System Technical Journal, 46(9):2111-2118, 1967. 